\title{
Geometric endoscopy and mirror symmetry
}

\author{
EDWARD FRENKEL AND EdWARd WitTEN
}

\begin{abstract}
The geometric Langlands correspondence has been interpreted as the mirror symmetry of the Hitchin fibrations for two dual reductive groups. This mirror symmetry, in turn, reduces to $T$-duality on the generic Hitchin fibers, which are smooth tori. In this paper, we study what happens when the Hitchin fibers on the $B$-model side develop orbifold singularities. These singularities correspond to local systems with finite groups of automorphisms. In the classical Langlands program, local systems of this type are called endoscopic. They play an important role in the theory of automorphic representations, in particular, in the stabilization of the trace formula. Our goal is to use the mirror symmetry of the Hitchin fibrations to expose the special role played by these local systems in the geometric theory. The study of the categories of $A$-branes on the dual Hitchin fibers allows us to uncover some interesting phenomena associated with the endoscopy in the geometric Langlands correspondence. We then follow our predictions back to the classical theory of automorphic functions. This enables us to test and confirm them. The geometry we use is similar to that which is exploited in recent work by Ngô, a fact which could be significant for understanding the trace formula.
\end{abstract}

\section{Introduction}

This paper is concerned with some natural questions arising in the study of Langlands duality and mirror symmetry. On the mathematics side, the question is to describe a geometric analog of the phenomenon of endoscopy in the theory of automorphic representations. On the physics side, it is to explore the limit of the $T$-duality of supersymmetric sigma models with smooth dual tori as the target manifolds when the tori become singular.

Somewhat surprisingly, the two questions turn out to be closely related. The reason is that, according to [30,39], the geometric Langlands correspondence may be interpreted in terms of the mirror symmetry of the Hitchin 
fibrations for two dual reductive groups, $G$ and ${ }^{\mathrm{L}} G$ :

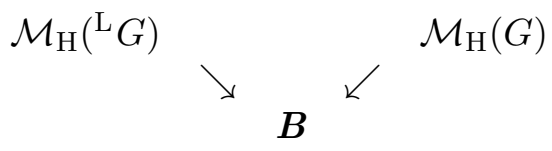

Here $\mathcal{M}_{\mathrm{H}}(G)$ denotes the moduli space of Higgs $G$-bundles on a smooth Riemann surface $C$, and $\boldsymbol{B}$ is the common base of the corresponding two dual Hitchin fibrations $[33,34]$. The mirror symmetry between them is realized via the fiberwise $T$-duality, in the framework of the general StromingerYau-Zaslow picture [73]. This duality is also closely related to the $S$-duality of certain supersymmetric four-dimensional gauge theories corresponding to $G$ and ${ }^{\mathrm{L}} G[39]$.

\subsection{T-duality of singular fibers}

The generic fibers of the Hitchin fibrations are smooth dual tori (which may be described as generalized Prym varieties of spectral curves when $\left.G=S L_{n}\right)$, and the $T$-duality is relatively well understood for these smooth fibers. In particular, it sets up an equivalence between the category of $B$-branes on the fiber ${ }^{\mathrm{L}} \boldsymbol{F}_{b}$ of $\mathcal{M}_{\mathrm{H}}\left({ }^{\mathrm{L}} G\right)$ at $b \in \boldsymbol{B}$ and the category of $A$ branes on the dual fiber $\boldsymbol{F}_{b}$ (more precisely, their connected components). The simplest $B$-branes are the skyscraper coherent sheaves supported at the points of ${ }^{\mathrm{L}} \boldsymbol{F}_{b} .{ }^{1}$ Under this equivalence of categories, they correspond to the $A$-branes which are rank one unitary flat bundles on $\boldsymbol{F}_{b}$. The latter have an important property: they are eigenbranes of certain operators which are twodimensional shadows of the 't Hooft line operators of the four-dimensional gauge theory [39] and are closely related to the Hecke correspondences on $G$-bundles. This property is dual to the more easily established property of the skyscraper $B$-branes of being eigenbranes of the so-called Wilson operators [39].

It is natural to ask: what does the $T$-duality look like at the singular fibers of the Hitchin fibrations? In particular, where does the $T$-duality map the $B$-branes supported at the singular points of $\mathcal{M}_{\mathrm{H}}\left({ }^{\mathrm{L}} G\right)$ ? In this paper, we consider the case that the singularity of the fiber is the mildest possible, namely, an orbifold singularity. (For example, in the case of $S L_{n}$, these are the only singularities if the spectral curve is irreducible and reduced.) This

\footnotetext{
${ }^{1}$ The category of $B$-branes should be considered here in the complex structure $J$, in which $\mathcal{M}_{\mathrm{H}}\left({ }^{\mathrm{L}} G\right)$ is realized as the moduli space of flat ${ }^{\mathrm{L}} G$-bundles. However, skyscraper sheaves are legitimate $B$-branes in any complex structure.
} 
turns out to be precisely the situation of "elliptic endoscopy," as defined in $[64,65]$ (see below). In the present paper, we describe in detail what happens in the case of the group $G=S L_{2}$ and explain how to generalize our results to other groups.

In the case of $G=S L_{2}$, the singular points of $\mathcal{M}_{\mathrm{H}}\left({ }^{\mathrm{L}} G\right)$ that we are interested in correspond to the ${ }^{\mathrm{L}} \mathrm{G}=\mathrm{SO}_{3}$ local systems (or Higgs bundles) on the curve $C$ which are reduced to the subgroup $\mathrm{O}_{2} \subset \mathrm{SO}_{3}$ (this is the simplest possible scenario for elliptic endoscopy, as explained below).Generic local systems of this type have the group of automorphisms $\mathbb{Z}_{2}$ (which is the center of $\mathrm{O}_{2}$ ) and therefore the corresponding points of $\mathcal{M}_{\mathrm{H}}\left({ }^{\mathrm{L}} G\right)$ are really $\mathbb{Z}_{2}$-orbifold points. This means that the category of $B$-branes supported at such a point is equivalent to the category $\operatorname{Rep}\left(\mathbb{Z}_{2}\right)$ of representations of $\mathbb{Z}_{2}$. Thus, it has two irreducible objects. Therefore, we expect that the dual category of $A$-branes should also have two irreducible objects. In fact, we show that the dual Hitchin fiber has two irreducible components in this case and the sought-after $A$-branes are the so-called fractional branes supported on these two components. Only their sum (or union) is an eigenbrane of the 't Hooft-Hecke operators (reflecting the fact that the sole eigenbrane of the Wilson operators in the $B$-model corresponds to the regular representation of $\mathbb{Z}_{2}$, that is, the direct sum of its two irreducible representations). However, we show that each of the two fractional $A$-branes separately satisfies a certain natural modification of the standard Hecke property (the "fractional Hecke property"), which has a direct generalization to other groups and is of independent interest.

\subsection{A-branes and $\mathcal{D}$-modules}

In the conventional formulation of the geometric Langlands correspondence [25, Section 6], the objects corresponding to ${ }^{\mathrm{L}} G$-local systems on $C$ are the so-called Hecke eigensheaves. These are $\mathcal{D}$-modules (or perverse sheaves) on the moduli stack $\operatorname{Bun}_{G}$ of $G$-bundles on $C$ satisfying the Hecke property. However, their structure is notoriously complicated and it is difficult to analyze them explicitly. In contrast, in the new formalism developed in $[30,39]$, the Hecke eigensheaves are replaced by the $A$-branes which are eigenbranes of the 't Hooft operators. These $A$-branes are much easier to "observe experimentally" and to analyze explicitly. Our idea is to use this new language in order to gain insights into the structure of the geometric Langlands correspondence - specifically, the part that pertains to endoscopy (and, more ambitiously, to the general functoriality principle). 
The passage from $A$-branes on $\mathcal{M}_{\mathrm{H}}(G)$ to $\mathcal{D}$-modules on $\operatorname{Bun}_{G}$ was explained in [39] (see [61,62] for a possible alternative approach; also, see $[1,17]$, where $\mathcal{D}$-modules have been introduced in physics from a different point of view). Although this has not yet been made completely rigorous mathematically, it is sufficient to describe important characteristics of the Hecke eigensheaves associated to eigenbranes, such as their reducibility, the open subsets of $\operatorname{Bun}_{G}$ where the Hecke eigensheaves are represented by local systems, the ranks of these local systems, and even their monodromy. Thus, our results on $A$-branes have direct implications for Hecke eigensheaves. In particular, if an eigenbrane $\mathcal{A}$ decomposes into two irreducible branes $\mathcal{A}_{1}$ and $\mathcal{A}_{2}$, then we predict that the corresponding Hecke eigensheaf $\mathcal{F}$ will also decompose as a direct sum of two $\mathcal{D}$-modules, $\mathcal{F}_{1}$ and $\mathcal{F}_{2}$, corresponding to $\mathcal{A}_{1}$ and $\mathcal{A}_{2}$, respectively. Furthermore, these two $\mathcal{D}$-modules should then separately satisfy the fractional Hecke eigensheaf property alluded to above.

\subsection{From curves over $\mathbb{C}$ to curves over $\mathbb{F}_{q}$}

The upshot of all this is that by analyzing the categories of $A$-branes supported on the singular Hitchin fibers, we gain insight into the geometric Langlands correspondence. We then make another leap of faith and postulate that the same structures on the Hecke eigensheaves that we observe for curves over $\mathbb{C}$ (such as their decomposition into two direct summands) should also hold for curves over finite fields. In the latter case, to a fractional Hecke eigensheaf, we may associate an automorphic function on the adèlic group $G\left(\mathbb{A}_{\mathrm{F}}\right)$ by taking the traces of the Frobenius on the stalks (this is referred to as the Grothendieck faisceaux-fonctions dictionnaire, see Section 8.1). Our predictions for the fractional Hecke eigensheaves then get translated into concrete predictions for the behavior of these automorphic functions under the action of the classical Hecke operators. We show that functions satisfying these properties do exist, and this provides a consistency check for our conjectures.

Thus, our starting point is the homological mirror symmetry between the categories of branes on the dual Hitchin fibrations. By applying the following sequence of transformations:

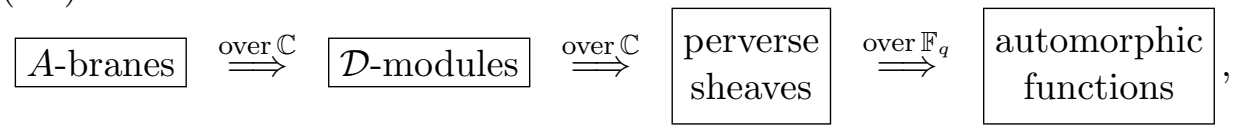


we link the structure of $A$-branes that we observe in the study of this mirror symmetry to the classical theory of automorphic forms. From this point of view, the $A$-branes that are eigenbranes of the 't Hooft-Hecke operators (in the ordinary sense) are geometric analogs of the Hecke eigenfunctions that encapsulate irreducible automorphic representations. This leads to a tantalizing question: what is the representation theoretic analog of the fractional eigenbranes into which the $A$-eigenbranes break in the endoscopic case, when the Hitchin fiber becomes singular? Langlands [46] has previously suggested a nice analogy between irreducible automorphic representations and elementary particles [46]. From this point of view, the existence of fractional $A$-branes indicates the existence of some inner, "quark-like," structure of automorphic representations, which is still waiting to be fully explored and understood.

We hope that understanding these structures will give us important clues about the geometric meaning of endoscopy.

\subsection{Classical endoscopy}

Endoscopy is one of the most fascinating phenomena in the classical Langlands correspondence. To motivate it, let us recall (see, e.g., [25, Section 2.4], for more details) the Langlands correspondence for the group $G L_{n}$. Let $C$ be a smooth projective curve over a finite field $\mathbb{F}_{q}$ and $F$ the field of rational functions on $C$. The Weil group $W_{F}$ is a dense subgroup of the Galois group $\operatorname{Gal}(\bar{F} / F)$ of automorphisms of the (separable) closure $\bar{F}$ of $F$. The Langlands correspondence sets up a bijection between $n$-dimensional ( $\ell$-adic) representations of the Weil group $W_{F}$ and irreducible automorphic representations of $G L_{n}\left(\mathbb{A}_{F}\right)$, where $\mathbb{A}_{F}$ is the ring of adèles of $F[22,23,43] .^{2}$ If we replace $G L_{n}$ by a more general reductive group $G$, then, in the first approximation, we should expect that irreducible automorphic representations of $G(\mathbb{A})$ would be in bijection with ( $\ell$-adic) homomorphisms $\sigma$ from $W_{F}$ to the Langlands dual group ${ }^{\mathrm{L}} G$ of $G$. However, it turns out that in general to each $\sigma$ corresponds not one, but several (possibly infinitely many), irreducible automorphic representation of the adèlic group $G\left(\mathbb{A}_{\mathrm{F}}\right)$. The set of equivalence classes of these representations is called the $L$-packet associated to $\sigma$, after the work of Labesse-Langlands [42] in which this phenomenon was discovered (for the group $G=S L_{2}$ ).

\footnotetext{
${ }^{2}$ This is the Langlands correspondence for the function fields. There is a similar, but more complicated, number fields version, in which $F$ is replaced by the field $\mathbb{Q}$ of rational numbers or its finite extension; see, e.g., [25, Part I] for more details.
} 
The structure of the $L$-packets is most interesting in the case of homomorphisms $W_{F} \rightarrow{ }^{\mathrm{L}} G=\mathrm{SO}_{3}$ that have their image contained in the subgroup $\mathrm{O}_{2} \subset P G L_{2}$, but not in its connected component $\mathrm{SO}_{2}$ which implies that their group of automorphisms is disconnected (generically, it is $\mathbb{Z}_{2}$ ). Thus, the automorphic representation theory of $S L_{2}\left(\mathbb{A}_{\mathrm{F}}\right)$ is governed in part by the group $\mathrm{O}_{2}$. This group (or rather, to keep with the traditional terminology, the subgroup $H$ of $S L_{2}(F)$ whose dual is $O_{2}$ ) is an example of an endoscopic group, and this relation is an example of the mysterious phenomenon known as the "endoscopy." It was discovered by Langlands and others in their attempt to organize automorphic representations in a way that would be compatible with the structure of the orbital integrals appearing on the geometric side of the trace formula.

Let us explain this briefly, referring the reader to $[5,40,45]$ for more details. The trace formula, or rather, its "regular elliptic part" (to which we will restrict ourselves here), has the following general form:

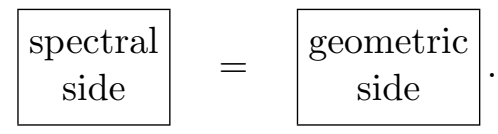

The spectral side is equal to the sum of traces of a test function $f$ with compact support on $G\left(\mathbb{A}_{\mathrm{F}}\right)$ over irreducible tempered cuspidal automorphic representations of $G\left(\mathbb{A}_{\mathrm{F}}\right)$ (we recall that those are realized in a certain space of functions on the quotient $\left.G(F) \backslash G\left(\mathbb{A}_{\mathrm{F}}\right)\right)$ :

$$
\sum_{\sigma: W_{F} \rightarrow{ }^{\mathrm{L}} G} \sum_{\phi \in L_{\sigma}} m_{\phi} \operatorname{Tr}\left(f, \pi_{\phi}\right) .
$$

Here the sum is over a certain class of homomorphisms $\sigma: W_{F} \rightarrow{ }^{\mathrm{L}} G$, which are supposed to label the $L$-packets $L_{\sigma}$ of equivalence classes of irreducible automorphic representations $\left\{\pi_{\phi}\right\}_{\phi \in L_{\sigma}}$, and $m_{\phi}$ denotes the multiplicity of $\pi_{\phi}$ in the space of automorphic functions.

The geometric side is the sum of orbital integrals of $f$, that is, integrals of $f$ over $G\left(\mathbb{A}_{\mathrm{F}}\right)$-conjugacy classes of elements of $G(F)$.

The geometric side needs to be "stabilized." This means rewriting it as a sum of integrals over stable conjugacy classes of elements of $G(F)$ in $G\left(\mathbb{A}_{\mathrm{F}}\right){ }^{3}$ This is necessary for many reasons, one of which is that without this one

\footnotetext{
${ }^{3}$ Two elements of $G(k)$, where $k$ is any field, are called stably conjugate if they are conjugate in $G(\bar{k})$. Since $\mathbb{A}_{\mathrm{F}}$ is the restricted product of completions of $F$, we obtain a natural notion of stable conjugacy in $\mathbb{A}_{F}$ as well.
} 
cannot even hope to compare the geometric sides of the trace formulas for different groups [5, Section 27]. The resulting expression for the geometric side reads $[45]$

$$
\sum_{\mathrm{H}} \imath(H, G) \mathrm{ST}_{\mathrm{ell}}^{G-\mathrm{reg}}\left(f^{H}\right)
$$

where the sum is over the elliptic endoscopic groups $H$ of $G$ (they are not subgroups of $G$ in general), as well as $H=G$ itself, $\mathrm{ST}_{\text {ell }}^{G-r e g}\left(f^{H}\right)$ denotes the sum of stable orbital integrals for the group $H$ and the $\imath(H, G)$ are certain numbers. The elliptic endoscopic groups are defined, roughly, as the dual groups of the centralizers of semi-simple elements in ${ }^{\mathrm{L}} G$ which are not contained in any proper Levi subgroups of ${ }^{\mathrm{L}} G$.

Formula (1.4) hinges upon a number of assumptions, the most important of which is the so-called transfer conjecture. It states the existence of an assignment $f \mapsto f^{H}$, from functions on $G\left(\mathbb{A}_{\mathrm{F}}\right)$ to those on $H\left(\mathbb{A}_{\mathrm{F}}\right)$, satisfying the property that, roughly speaking, the stable orbital integrals of $f^{H}$ are equal to stable orbital integrals of $f$ modified by a certain twist (see $[5,14,45]$ and references therein for details).

A special case of the transfer conjecture (when $f$ is the characteristic function of a maximal compact subgroup of $G$; then $f^{H}$ is required to be of the same kind) is the so-called fundamental lemma. The fundamental lemma (in the function field case) has been recently proved by Ngô [65] (see also $[29,52,53,64])$. More precisely, Ngô has proved a Lie algebra version of the fundamental lemma, but Waldspurger has shown that it implies the fundamental lemma for the group, as well as the general transfer conjecture [74]. Also, the fundamental lemma in the function field case that we are discussing is equivalent to the one in the number field case, provided that the characteristics of the residue fields are sufficiently large $[13,75]$.

Since the geometric side of the trace formula has the form (1.4), it is natural to expect that the spectral side has a similar form, that is, it may be written as a sum of terms labeled by the elliptic endoscopic groups, with individual terms on both sides of (1.2) being equal. A formula of this sort has been first established by Labesse-Langlands for $G=S L_{2}$ (and its inner forms). In general, the corresponding formula was conjectured by Kottwitz [40]. This formula has a number of important consequences for the theory of automorphic representations. First of all, it leads to an explicit formula for the multiplicities $m_{\phi}$ of the automorphic representations in the $L$-packet associated to a homomorphism $\sigma: W_{F} \rightarrow{ }^{\mathrm{L}} G$ (appearing in (1.3)). The answer is a linear combination of terms associated to 
the elliptic endoscopic groups $H$ such that the image of $\sigma$ is contained in ${ }^{\mathrm{L}} H \subset{ }^{\mathrm{L}} G$.

Perhaps it would be helpful to explain here the relation between $\sigma$ and the endoscopic groups. Suppose for simplicity that ${ }^{\mathrm{L}} G$ is a semi-simple group of adjoint type (so it has trivial center), and the image of a homomorphism $\sigma: W_{F} \rightarrow{ }^{\mathrm{L}} G$ occurring in (1.3) has finite centralizer $S_{\sigma}$. Then the Langlands duals ${ }^{\mathrm{L}} H$ of the endoscopic groups $H$ associated to $\sigma$ are just the centralizers of nontrivial elements $s \in S_{\sigma}$ in ${ }^{\mathrm{L}} G$ (hence the image of $\sigma$ is automatically contained in ${ }^{\mathrm{L}} H$ ). For instance, if ${ }^{\mathrm{L}} G=\mathrm{SO}_{3}$, the only subgroup that we can obtain this way is $\mathrm{O}_{2} \subset \mathrm{SO}_{3}{ }^{4}$

The second, and perhaps, more important, consequence of the transfer conjecture and the stabilized trace formula is that it gives a concrete realization of the Langlands functoriality principle [44] for the homomorphisms ${ }^{\mathrm{L}} H \rightarrow{ }^{\mathrm{L}} G$, where $H$ are the elliptic endoscopic groups. Namely, we obtain a natural map from $L$-packets of automorphic representations of $H\left(\mathbb{A}_{\mathrm{F}}\right)$ to those of $G\left(\mathbb{A}_{\mathrm{F}}\right)$ (see, e.g., $[4,5]$, Section 26 , for more details).

In short, the classical endoscopy establishes an elusive connection between automorphic representations of $G\left(\mathbb{A}_{F}\right)$ and those of its endoscopic groups $H\left(\mathbb{A}_{\mathrm{F}}\right)$ which matches, via the trace formula, the relation between orbital integrals for the two groups provided by the transfer conjecture.

\subsection{Geometric endoscopy}

In the last 20 years, significant progress has been made in translating the classical Langlands correspondence to the language of geometry. The emerging geometric Langlands correspondence has the advantage that it makes sense not only for curves defined over finite fields, but also for curves over the complex field, that is, Riemann surfaces. In this version, we have the opportunity to use the vast resources of complex algebraic geometry and thereby advance our understanding of the general Langlands duality patterns.

For many concepts of the classical Langlands, corresponding counterparts have been found in the geometric version. One important phenomenon that has not yet been understood geometrically is the endoscopy. It is the goal of this paper to make the first steps in the development of geometric endoscopy, by which we mean exposing the special role played by the endoscopic groups in the geometric Langlands correspondence. Since there is

\footnotetext{
${ }^{4}$ Note that as in $[64,65]$ and contrary to the standard convention, we do not consider here $G$ itself as an elliptic endoscopic group.
} 
no obvious analog of the trace formula in the geometric setting, we are not trying to imitate the stabilization of the trace formula that leads to the classical endoscopy. Rather, we wish to describe the new structures that emerge in the geometric Langlands correspondence for the ${ }^{\mathrm{L}} G$-local systems of elliptic endoscopic type. When ${ }^{\mathrm{L}} G$ is semi-simple, this means (by analogy with the classical setting) that the group of automorphisms of this local system is a finite group that is strictly larger than the center of ${ }^{\mathrm{L}} G$. For example, for ${ }^{\mathrm{L}} \mathrm{G}=\mathrm{SO}_{3}$, these are the local systems whose image is contained in $\mathrm{O}_{2} \subset \mathrm{SO}_{3}$, but not in the maximal torus of $\mathrm{O}_{2}$. Then the group of automorphisms is equal to $\mathbb{Z}_{2}$, the center of $\mathrm{O}_{2}$, unless the image is contained in a subgroup $\mathbb{Z}_{2} \times \mathbb{Z}_{2}$ (we will mostly ignore this case in the present paper).

Our approach is to use mirror symmetry of the Hitchin fibrations associated to $G$ and ${ }^{\mathrm{L}} G$ and to explore the structure of the $A$-branes corresponding to the endoscopic ${ }^{\mathrm{L}} G$-local systems (viewed as orbifold points of $\mathcal{M}_{\mathrm{H}}\left({ }^{\mathrm{L}} G\right)$, on the $B$-model side), which are realized on the corresponding singular Hitchin fibers in $\mathcal{M}_{\mathrm{H}}(G)$ (on the $A$-model side). The first advantage of this approach is that the endoscopic groups (which are rather mysterious in the classical theory, where they arise in the process of stabilization of the trace formula for the group $G$ ) are manifest: they occur naturally on the $B$ model side of mirror symmetry. Indeed, for any subgroup ${ }^{\mathrm{L}} H \subset{ }^{\mathrm{L}} G$, there is a natural embedding $\mathcal{M}_{\mathrm{H}}\left({ }^{\mathrm{L}} H\right) \hookrightarrow \mathcal{M}_{\mathrm{H}}\left({ }^{\mathrm{L}} G\right)$, and a point of $\mathcal{M}_{\mathrm{H}}\left({ }^{\mathrm{L}} G\right)$ corresponding to an endoscopic local system $\mathcal{E}$ belongs to the image of $\mathcal{M}_{\mathrm{H}}\left({ }^{\mathrm{L}} H\right)$ for all endoscopic groups $H$ associated to it (i.e., those for which ${ }^{\mathrm{L}} H$ contains the image of $\mathcal{E}$ ). The second advantage, already mentioned above, is that, at least in the generically regular semi-simple case, the corresponding $A$-branes have a simple and transparent structure (in contrast to Hecke eigensheaves), and this simplifies our analysis considerably.

We then interpret the structures that we observe in the category of $A$-branes (on the $A$-model side) in terms of $\mathcal{D}$-modules (for curves over $\mathbb{C}$ ) or perverse sheaves (for curves over $\mathbb{C}$ or over $\mathbb{F}_{q}$ ) on $\operatorname{Bun}_{G}$, which are the more standard objects in the geometric Langlands correspondence. ${ }^{5}$ Finally, for curves over $\mathbb{F}_{q}$, we study the automorphic functions associated to these perverse sheaves (see diagram (1.1)). This results in a series of concrete predictions:

- Our first prediction is that the Hecke eigensheaves corresponding to an elliptic endoscopic ${ }^{\mathrm{L}} G$-local system $\mathcal{E}$ with the finite group of automorphisms $\Gamma$ splits into a direct sum of irreducible sheaves $\mathcal{F}_{R}$ labeled

\footnotetext{
${ }^{5}$ Alternatively, one may look at it from the point of view of a non-abelian version of the Fourier-Mukai transform [51,66], suggested by Beilinson and Drinfeld.
} 
by irreducible representations $R$ of $\Gamma$, with the multiplicity of $\mathcal{F}_{R}$ equal to $\operatorname{dim} R$.

- Our second prediction is that the sheaves $\mathcal{F}_{R}$ satisfy a fractional Hecke property described in Section 6. This prediction is confirmed in the case of curves over $\mathbb{F}_{q}$ : we check that the functions assigned to our sheaves and satisfying the function-theoretic analog of the fractional Hecke property do exist. Moreover, we express them as linear combinations of the ordinary Hecke eigenfunctions by a kind of Fourier transform over $\Gamma{ }^{6}$ Thus, it turns out that in the endoscopic case the functions assigned to irreducible perverse sheaves are not Hecke eigenfunctions, but linear combinations thereof.

- Our third prediction is that if the image $b$ of $\mathcal{E}$ in the Hitchin base $\boldsymbol{B}$ is a generically regular semi-simple point, then the group $\Gamma$ is abelian and is isomorphic to a subgroup of the dual group of $\pi_{0}\left(\boldsymbol{P}_{b}\right)$, where $\boldsymbol{P}_{b}$ is the generalized Prym of $b$. Furthermore, there exists a local system in the dual Hitchin fiber ${ }^{\mathrm{L}} \boldsymbol{F}_{b}$ for which $\Gamma$ is isomorphic to the dual group of $\pi_{0}\left(\boldsymbol{P}_{b}\right)$ (note that $\pi_{0}\left(\boldsymbol{P}_{b}\right)$ acts simply transitively on an open dense subset of the Hitchin fiber $\boldsymbol{F}_{b}$ over $b$ in $\mathcal{M}_{\mathrm{H}}(G)$, which is reduced in this case $[65]) .^{7}$

We hope that proper understanding of these phenomena will lead to better understanding of endoscopy and related subjects such as the fundamental lemma.

\subsection{Connection with the work of Ngô}

A link between our analysis and the classical endoscopy comes from the fact that the geometry we use is similar to what is exploited in the recent work of Ngô [64,65] (see also the excellent survey [14]). Ngô has discovered a striking connection between the orbital integrals appearing on the geometric side of the trace formula (1.2) and the cohomology of the Hitchin fibers in moduli space $\mathcal{M}_{\mathrm{H}}(G)$ (more precisely, in generalized versions of $\mathcal{M}_{\mathrm{H}}(G)$ which parametrize meromorphic Higgs fields with the divisor of poles $D$ which is sufficiently large). He used it to prove the fundamental lemma, in the Lie algebra setting, for function fields (for unitary groups, he had done it earlier

\footnotetext{
${ }^{6}$ This is somewhat reminiscent of the Fourier transform observed by Lusztig [55] in the theory of character sheaves.

${ }^{7}$ In the course of writing this paper, we were informed by Ngô that he was also aware of this statement.
} 
together with Laumon [53]). He achieved that by interpreting the orbital integrals as numbers of points of the Hitchin fibers $\boldsymbol{F}_{b}$ in the moduli stack of Higgs bundles $\mathcal{M}_{\mathrm{H}}(G)$ defined for a curve over a finite field. These numbers are in turn interpreted as traces of the Frobenius acting on the ( $\ell$-adic) cohomology of $\boldsymbol{F}_{b}$. The crucial step in Ngô's construction is the decomposition of this cohomology with respect to the action of the finite abelian group $\pi_{0}\left(\boldsymbol{P}_{b}\right)$, where $\boldsymbol{P}_{b}$ is the generalized Prym variety associated to an elliptic point $b \in \boldsymbol{B}$. He identified the $\kappa$-isotypic part of this decomposition, where $\kappa$ is a character of $\pi_{0}\left(\boldsymbol{P}_{b}\right)$, with the $\kappa$-part of the decomposition of the cohomology of the corresponding Hitchin fiber of the endoscopic group $H_{\kappa}$. Taking traces of the Frobenius over these subspaces, he obtained the fundamental lemma. (Here we should mention the earlier works $[29,52]$ in which closely related geometric interpretations of the fundamental lemma had been given.)

Thus, Ngô uses geometry that seems very close to the geometry we are using. Indeed, we consider the fractional $A$-branes on $\mathcal{M}_{\mathrm{H}}(G)$, supported on the Hitchin fiber $\boldsymbol{F}_{b}$, which are essentially labeled by $\pi_{0}\left(\boldsymbol{P}_{b}\right)$, and Ngô considers the cohomology of similarly defined Hitchin fibers and their decomposition under $\pi_{0}\left(\boldsymbol{P}_{b}\right)$. However, there are important differences.

First of all, we work over $\mathbb{C}$, whereas Ngô works over $\mathbb{F}_{q}$. In the latter setting, there is no obvious analog of the homological mirror symmetry between $\mathcal{M}_{\mathrm{H}}(G)$ and $\mathcal{M}_{\mathrm{H}}\left({ }^{\mathrm{L}} G\right)$ that is crucial to our approach. (In fact, neither the dual group ${ }^{\mathrm{L}} G$ nor the dual Hitchin moduli space $\mathcal{M}_{\mathrm{H}}\left({ }^{\mathrm{L}} G\right)$ plays a role in Ngô's work.) Second, Ngô works with generalized moduli spaces of Higgs bundles labeled by effective divisors $D$ on the curve $C$. Our moduli space $\mathcal{M}_{\mathrm{H}}(G)$ corresponds to a divisor of the canonical line bundle on $C{ }^{8}$ Third, and most importantly, the objects we assign to the connected components of the singular Hitchin fiber $\boldsymbol{F}_{a}$, the $A$-branes, are objects of automorphic nature; we hope to relate them to Hecke eigensheaves and ultimately to the automorphic functions in the classical theory. Thus, these objects should live on the spectral side of the trace formula (1.2). On the other hand, Ngô relates the numbers of points of the Hitchin fibers (and their cohomology) to the orbital integrals appearing on the geometric side of the trace formula (more precisely, its Lie algebra version). A priori, this has nothing to do with automorphic representations (or automorphic functions). The connection between orbital integrals and automorphic representations

\footnotetext{
${ }^{8}$ Technically, this case is outside of the scope of Ngô's work, since he imposes the condition $\operatorname{deg}(D)>2 g-2$. However, as he explained to us, most of his results remain true when $D$ is a divisor of the canonical line bundle.
} 
is provided by the trace formula, but in a rather indirect, combinatorial way. It arises only when we sum up all orbital integrals on the geometric side, and over all representations on the spectral side of the trace formula.

This raises the following question: could there be a direct link between individual Hitchin fibers in the moduli space $\mathcal{M}_{\mathrm{H}}(G)$ over $\mathbb{F}_{q}$ and individual automorphic representations? After all, we have a natural forgetful map from $\mathcal{M}_{\mathrm{H}}(G)$ to $\operatorname{Bun}_{G}$, where unramified automorphic functions live. Could it be that the passage from $A$-branes to Hecke eigensheaves discussed above has an analog in the classical theory as a passage from orbital integrals to Hecke eigenfunctions?

In any case, we find it remarkable that the same geometry of the Hitchin fibration that Ngô has used to understand the geometric side of the trace formula is also used in our study of the geometric endoscopy via mirror symmetry and therefore appears to be pertinent to automorphic representations (via the correspondence (1.1)). This connection could potentially be significant as it could shed new light on the trace formula and the theory of automorphic representations in general. ${ }^{9}$

\subsection{Quantum field theory}

Finally, we wish to relate our computation to various issues in quantum field theory. Recall that the Strominger-Yau-Zaslow picture [73] relates homological mirror symmetry of two manifolds $X$ and $Y$ to the $T$-duality of dual special Lagrangian fibrations in $X$ and $Y$. This works especially nicely for the generic fibers, which are smooth. The $T$-duality of these fibers may be thought of as a kind of abelian version of mirror symmetry (closely related to the Fourier-Mukai transform). Therefore, important "non-abelian" information about the mirror symmetry of $X$ and $Y$ is hidden in the duality of the singular fibers. Special Lagrangian fibrations are difficult to understand in general, but in the case of Hitchin fibrations, the hyper-Kahler structure leads to a drastic simplification [32] which we will exploit to analyze certain cases of singular fibers. In particular, we show that under mirror symmetry, the orbifold singularities on $X$ correspond to reducible Lagrangian fibers in $Y$; we believe that this is a fairly general phenomenon. From this point of view, the geometric endoscopy appears as a special case of mirror symmetry in the presence of orbifold singularities.

\footnotetext{
${ }^{9}$ This seems to resonate with the views expressed by R. Langlands [47] in his recent Shaw Prize lecture.
} 
Geometric endoscopy might have a natural realization physically by means of a supersymmetric domain wall, with $\mathcal{N}=4$ supersymmetric YangMills theory of gauge group ${ }^{\mathrm{L}} H$ on one side of the domain wall, and the same theory with gauge group ${ }^{\mathrm{L}} G$ on the other side (with $H$ being an endoscopic group of $G$ ). This domain wall should in particular give rise after duality to a functor from the category of $A$-branes on $\mathcal{M}_{\mathrm{H}}(H)$ to the category of $A$-branes on $\mathcal{M}_{\mathrm{H}}(G)$. This is a geometric analog of the notion of transfer, or the functoriality principle, in the Langlands Program (see Section 5.2.6).

Finally, as we explain in Section 3.11, the Hitchin fibrations studied in this paper also appear in Seiberg-Witten theory. In that context, the important four-manifold invariants arise as contributions from the endoscopic points. The appearance of the same Hitchin fibrations in the two different problems can be traced to an underlying six-dimensional quantum field theory that can be compactified to four dimensions in two different ways.

\subsection{Plan of the paper}

In Section 2, we give an overview of the connection between the homological mirror symmetry of the dual Hitchin fibrations and the geometric Langlands program. In Section 3, we take up our main example: the moduli spaces of $\mathrm{SL}_{2}$ and $\mathrm{SO}_{3}$ Higgs bundles on an elliptic curve with tame ramification at one point. We describe in detail these moduli spaces, their singularities and the corresponding categories of branes. This example will serve as the prototype for the general picture developed in this paper.

In Section 4, we discuss in more detail the passage from $A$-branes to $\mathcal{D}$-modules. Although we focus largely on the genus 1 example developed in the preceding section, many aspects of this discussion apply in a more general setting. Section 5 describes the generalization of our results to curves of higher genus. We also compute explicitly the action of the Wilson and 't Hooft/Hecke operators on the electric and magnetic branes relevant to geometric endoscopy. In Section 6, we explain how these results fit in a general categorical formalism. In particular, we introduce the notion of "fractional Hecke eigensheaves" and conjecture that the $\mathcal{D}$-modules associated to the fractional $A$-branes found in Section 5 are objects of this type.

Our next task is to describe the analogs of these conjectures in the case when our curve $C$ is defined over a finite field and to link them to the theory of automorphic functions. In Section 7, we recall the set-up of endoscopy and $L$-packets in the classical theory of automorphic forms. We focus in particular on the unramified case for $G=S L_{2}$ analyzed first by 
Labesse and Langlands [42]. We then discuss in Section 8 the potential implications for the classical theory of the geometric picture outlined in the earlier sections. In particular, we compute the automorphic functions associated to the fractional Hecke eigensheaves. We generalize our results and conjectures to other groups in Section 9. Finally, in Section 10, we discuss the tricky point that the two fractional eigenbranes that we have found in the case of $S L_{2}$ are indistinguishable. We trace this phenomenon to a certain $\mathbb{Z}_{2}$-gerbe that appears to be a subtle, but important, ingredient of the mirror symmetry of the Hitchin fibrations. In Appendix, we explain the structure of the unramified global $L$-packets for $S L_{2}$ in concrete terms using the Whittaker functions.

\section{Duality, branes and endoscopy}

\subsection{Geometric Langlands duality and mirror symmetry}

In the gauge theory approach, the geometric Langlands correspondence is understood as a mirror symmetry.

Let $\mathcal{Y}(C ; G)$ be the moduli space of flat $G$-bundles ${ }^{10}$ over an oriented two-manifold $C$. Here $G$ is a complex reductive Lie group. Then $\mathcal{Y}(C ; G)$ is in a natural way a complex symplectic manifold. The complex structure of $\mathcal{Y}(C ; G)$ comes simply from the complex structure of $G$, and its holomorphic two-form $\Omega$ is defined using the intersection pairing on the tangent space to $\mathcal{Y}(C ; G)$, which is $H^{1}(C, \operatorname{ad}(Y))$.

Using the complex structure of $\mathcal{Y}(C ; G)$ (and the triviality of its canonical bundle, which follows from the fact that $\mathcal{Y}(C ; G)$ is complex symplectic), one can define a $B$-model of $\mathcal{Y}(C ; G)$. Similarly, viewing $\mathcal{Y}(C ; G)$ as a real symplectic manifold, with symplectic structure $\omega=\operatorname{Im} \Omega$, one can define an $A$-model. ${ }^{11}$

${ }^{10}$ The complex form of a Lie group is always meant unless otherwise specified. This is in keeping with most literature on the geometric Langlands program, but in contrast to most literature on gauge theory including $[30,39]$.

${ }^{11}$ The definition of $\Omega$ is such that $\operatorname{Im} \Omega$ is cohomologically trivial, though $\operatorname{Re} \Omega$ is not. The definition of $\Omega$ depends on the choice of a nondegenerate quadratic form on $\mathfrak{g}$, the Lie algebra of $G$. However, the $A$-model and therefore the geometric Langlands duality derived from it is independent of this choice, up to a natural isomorphism. Once the relation between flat bundles and Higgs bundles [33] is incorporated, this follows from the $\mathbb{C}^{*}$ action on the moduli space of Higgs bundles. In the gauge theory approach [39], it is clear a priori that this choice is inessential, since the dependence of the action on the gauge coupling is of the form $\{Q, \cdot\}$. 
Now let ${ }^{L} G$ and $G$ be a dual pair of complex reductive Lie groups. It turns out that there is a mirror symmetry between the $B$-model of $\mathcal{Y}\left(C ;{ }^{\mathrm{L}} G\right)$ and the $A$-model of $\mathcal{Y}(C ; G)$. This instance of mirror symmetry was first studied by Hausel and Thaddeus [32]. (A closely related duality has been studied by Donagi and Pantev [20], Hitchin [35] and Arinkin [2]. The relation between the two is explained in section 5.3.) It was deduced from electric-magnetic duality of four-dimensional supersymmetric gauge theory in [39], following earlier arguments [9,31], and shown to underlie geometric Langlands duality. ${ }^{12}$

To establish mirror symmetry between $\mathcal{Y}\left(C ;{ }^{\mathrm{L}} G\right)$ and $\mathcal{Y}(C ; G)$, one needs the fact that these spaces have another interpretation as moduli spaces of Higgs bundles. This comes from Hitchin's equations. Unlike the complex symplectic structure of $\mathcal{Y}(C ; G)$, which we have considered hitherto, Hitchin's equations require a choice of conformal structure on $C$. So henceforth $C$ is a complex Riemann surface, not just an oriented two-manifold.

2.1.1. Hitchin's equations Hitchin's equations [33] are nonlinear equations for a pair $(A, \phi)$. $A$ is a connection on a $G$-bundle $E \rightarrow C$ with structure group the compact form of $G$. And $\phi$ is a one-form on $C$ that is valued in $\mathfrak{g}$, the Lie algebra of this compact form. Hitchin's equations read

$$
\begin{array}{r}
F-\phi \wedge \phi=0 \\
d_{A} \phi=d_{A} \star \phi=0 .
\end{array}
$$

Here $d_{A}$ is the gauge-covariant exterior derivative and $\star$ is the Hodge star operator.

If $(A, \phi)$ is a solution of Hitchin's equations, then $\mathcal{A}=A+i \phi$ is a complex-valued flat connection, and thus endows $E$ (or its complexification) with the structure of a local system. In particular, a solution of Hitchin's equations determines a point in $\mathcal{Y}(C ; G)$, the moduli space of local systems with structure group $G$.

Alternatively, the $\bar{\partial}$ operator given by the $(0,1)$ part of the operator $d_{A}$ endows $E$ with a holomorphic structure. And if we write $\varphi$ for the $(1,0)$ part of $\phi$ and $K$ for the canonical bundle of $C$, then $\varphi$ is a section of $K \otimes \operatorname{ad}(E)$ and is holomorphic according to Hitchin's equations. The pair

\footnotetext{
${ }^{12}$ We will restrict ourselves to the most basic form of the geometric Langlands duality. In [39], it is explained how one can incorporate in the gauge theory approach an additional complex parameter, leading to what in the mathematical literature is sometimes called quantum geometric Langlands.
} 
$(E, \varphi)$ then defines what is known from a holomorphic point of view as a Higgs bundle.

A basic result about Hitchin's equations is that the moduli space $\mathcal{M}_{\mathrm{H}}=$ $\mathcal{M}_{\mathrm{H}}(C ; G)$ of solutions of these equations is a hyper-Kahler manifold. In one complex structure, called $I$ in [33], $\mathcal{M}_{\mathrm{H}}$ is the moduli space of stable Higgs bundles, while in another complex structure, denoted as $J$, it is the moduli space of stable local systems and coincides with what we have earlier called $\mathcal{Y}$. The fact that the same space has these dual interpretations is one of the reasons that it is possible to say something about geometric Langlands by studying Higgs bundles.

As a hyper-Kahler manifold, $\mathcal{M}_{\mathrm{H}}$ has a distinguished triple $\omega_{I}, \omega_{J}$ and $\omega_{K}$ of real symplectic forms, which are Kahler, respectively, in complex structures $I, J$ and $K=I J$. Similarly, there are complex two-forms $\Omega_{I}=\omega_{J}+i \omega_{K}$ and $\Omega_{J}=\omega_{K}+i \omega_{I}$ and $\Omega_{K}=\omega_{I}+i \omega_{J}$ that are holomorphic symplectic forms, respectively, in complex structures $I, J$ and $K$. When $\mathcal{Y}$ is identified with $\mathcal{M}_{\mathrm{H}}$, its complex structure corresponds to $J$. Moreover, its holomorphic symplectic form is $\Omega=i \Omega_{J}$, and $\omega=\operatorname{Im} \Omega$ is equal to $\omega_{K}$.

2.1.2. The Hitchin fibration The Hitchin fibration is the map $\Pi: \mathcal{M}_{\mathrm{H}} \rightarrow \boldsymbol{B}$ that takes a Higgs bundle $(E, \varphi)$ to the characteristic polynomial of $\varphi$. ( $\boldsymbol{B}$ is a linear space that parametrizes the possible values of the characteristic polynomial.) The Hitchin fibration is holomorphic in complex structure $I$ (in which $\mathcal{M}_{\mathrm{H}}$ is the moduli space of Higgs bundles). The fibers of the fibration are Lagrangian submanifolds from the point of view of the holomorphic symplectic structure $\Omega_{I}$, and hence also from the point of view of $\omega_{K}=\operatorname{Im} \Omega_{I}$.

Thus, from the point of view of the $A$-model that is relevant in the geometric Langlands program, the Hitchin fibration is a fibration by Lagrangian submanifolds. These fibers generically are smooth tori (holomorphic in complex structure $I$ ). This fact is related to the interpretation of $\mathcal{M}_{\mathrm{H}}$ as a completely integrable Hamiltonian system [34] and can be seen explicitly using spectral curves, as we will explain in Section 5.

From the standpoint of the $A$-model, the fibers of the Hitchin fibration are not merely Lagrangian tori; they are special Lagrangian. Indeed, since they are holomorphic in one of the complex structures (namely $I$ ), they minimize the volume (computed using the hyper-Kahler metric of $\mathcal{M}_{\mathrm{H}}$ ) in their cohomology class. So [32] this is an example of a fibration by special Lagrangian tori, a geometric structure that has been proposed [73] to describe mirror symmetry. 
More specifically, if $G$ is simply laced, the bases of the Hitchin fibrations for $G$ and ${ }^{\mathrm{L}} G$ are the same. (For example, in Section 3, we will parametrize $\boldsymbol{B}$ by the same complex variable $w$ both for $G=S L_{2}$ and for ${ }^{\mathrm{L}} G=S O_{3}$.) For any $G$, there are natural isomorphisms between these bases. So we get a picture:

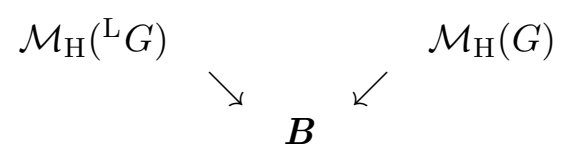

The fibers of the two fibrations over a generic point $b \in \boldsymbol{B}$ are dual tori, as first argued in [32] for $G=S L_{n}$. (For other groups, see [20,35].) This is the usual SYZ picture associated with mirror symmetry.

This particular example has several advantages. It is usually very difficult to concretely describe a special Lagrangian fibration, but in the case of $\mathcal{M}_{\mathrm{H}}$, the fact that the fibration is holomorphic in complex structure $I$ makes it accessible, as we will see in the examples discussed in Sections 3 and 5.3. Also, the relation between mirror symmetry and a special Lagrangian fibration is typically affected by what physicists call quantum corrections by disc instantons. However, the hyper-Kahler nature of $\mathcal{M}_{\mathrm{H}}$ ensures the absence of such corrections and makes more straightforward the application of the Hitchin fibration to this particular example of mirror symmetry.

\subsection{Branes and their duals}

To make contact with geometric Langlands duality, we must consider $B$-branes and $A$-branes on $\mathcal{M}_{\mathrm{H}}$.

A simple example of a $B$-brane $\mathcal{B}$ is a brane of rank 1 supported at a smooth point $r \in \mathcal{M}_{\mathrm{H}}\left(C ;{ }^{\mathrm{L}} G\right)$. Such a point corresponds to an irreducible ${ }^{\mathrm{L}} G$ local system $\mathcal{E}_{r}$ over $C$ (that is, one whose automorphism group reduces to the center of ${ }^{\mathrm{L}} G$; for the moment, assume that the center is trivial). It is contained in a fiber ${ }^{\mathrm{L}} \boldsymbol{F}_{b}$ of the Hitchin fibration of ${ }^{\mathrm{L}} G$ and lies over a point $b$ in the base $\boldsymbol{B}$ of the fibration. We let $\boldsymbol{F}_{b}$ denote the fiber over $b$ of the dual Hitchin fibration of $\mathcal{M}_{\mathrm{H}}(C ; G)$.

Mirror symmetry is understood as a $T$-duality on the fibers of the Hitchin fibration. So it maps $\mathcal{B}$ to an $A$-brane $\mathcal{A}$ whose support is $\boldsymbol{F}_{b}$, endowed with a flat unitary line bundle $\mathcal{L}_{r}$ that depends on $r$. This makes sense since the generic fibers ${ }^{\mathrm{L}} \boldsymbol{F}_{b}$ and $\boldsymbol{F}_{b}$ of the dual Hitchin fibrations are dual tori. So a point $r \in{ }^{\mathrm{L}} \boldsymbol{F}_{b}$ determines a flat unitary line bundle $\mathcal{L}_{r} \rightarrow \boldsymbol{F}_{b}$. 
What has just been described is the usual picture. What happens if $r$ is a singular point in $\mathcal{M}_{\mathrm{H}}\left(C ;{ }^{\mathrm{L}} G\right)$, corresponding to an ${ }^{\mathrm{L}} G$ local system that has a nontrivial automorphism group? The category of branes supported at a smooth point is equivalent to the category of vector spaces, via the map that takes a brane to its space of sections. The category of branes supported at a singular point is generally more complicated and cannot be reduced to a single object. Mirror symmetry or geometric Langlands duality must be applied to this whole category.

Suppose now that the automorphism group of the local system $\mathcal{E}_{r}$ is a nontrivial finite group $\Gamma$. In this case, the moduli space of ${ }^{\mathrm{L}} G$ local systems can be modeled near $r$ by a finite quotient $\mathbb{C}^{2 n} / \Gamma$ for some $n$, with a linear action of $\Gamma$ on $\mathbb{C}^{2 n}$ coming from a homomorphism $\Gamma \rightarrow S p_{2 n} \subset U_{2 n}$. ( $\Gamma$ acts via a subgroup of the symplectic group since $\mathcal{M}_{\mathrm{H}}$ is complex symplectic and in fact hyper-Kahler.) We identify $r$ with the origin in $\mathbb{C}^{2 n} / \Gamma$. The space of sections of a brane supported at $r$ is now a finite-dimensional vector space with an action of $\Gamma$. To describe a brane, we have to say how $\Gamma$ acts on this vector space. An irreducible brane corresponds to an irreducible representation. (For a more complete description, see Section 10.)

It makes sense to use this formalism even if $\Gamma$ is simply the center of ${ }^{\mathrm{L}} G$, in which case $r$ is a smooth point since the center acts trivially on the fields entering in Hitchin's equations. An irreducible representation of the center is one-dimensional, given by a character which in [39], section 7, was called $\boldsymbol{e}_{0}$. Thus the description of an irreducible brane at a smooth point can be refined to include specifying the character $\boldsymbol{e}_{0}$. Under duality, $\boldsymbol{e}_{0}$ maps to a characteristic class $\boldsymbol{m}_{0} \in H^{2}(C, G)$ that determines the topology of a $G$-bundle over $C$. A brane on $\mathcal{M}_{\mathrm{H}}\left(C ;{ }^{\mathrm{L}} G\right)$ of specified $e_{0}$ has a dual that is supported on an irreducible component of $\mathcal{M}_{\mathrm{H}}(C ; G)$ with definite $\boldsymbol{m}_{0}$.

However, endoscopy arises when the automorphism group is not simply the center, and we will illustrate the ideas assuming that the center of ${ }^{\mathrm{L}} G$ is trivial. For instance, in the example in Section 3, we will have ${ }^{\mathrm{L}} G=$ $S_{3}$, of trivial center. This being so, $\Gamma$ acts effectively on $\mathbb{C}^{2 n}$ and if $\Gamma$ is nontrivial, then $r$ is an orbifold singularity of $\mathcal{M}_{\mathrm{H}}\left(C ;{ }^{\mathrm{L}} G\right)$. In this case, for each isomorphism class of irreducible representation $R_{i}$ of $\Gamma$, there is a corresponding irreducible brane $\mathcal{B}_{i}$ supported at the point $r$.

Let $r^{*}$ be a smooth point of $\mathcal{M}_{\mathrm{H}}\left(C ;{ }^{\mathrm{L}} G\right)$, with irreducible brane $\mathcal{B}^{*}$. Now consider what happens as $r^{*}$ approaches an orbifold point $r$. In the limit, the irreducible brane $\mathcal{B}^{*}$ degenerates to the brane supported at $r$ and associated with the regular representation of $\Gamma$. (The reason for this is that a smooth point $r^{*} \in \mathbb{C}^{2 n} / \Gamma$ corresponds to a free $\Gamma$ orbit on $\mathbb{C}^{2 n}$; the functions on a free orbit furnish a copy of the regular representation.) The regular 
representation can be decomposed as $\bigoplus_{i} n_{i} R_{i}$, where the sum runs over all irreducible representations $R_{i}$ of $\Gamma$, and $n_{i}$ is the dimension of $R_{i}$. So the decomposition of $\mathcal{B}^{*}$ is

$$
\mathcal{B}^{*} \rightarrow \bigoplus_{i} n_{i} \mathcal{B}_{i}
$$

In the physics literature, this decomposition was first analyzed in [21], and the branes $\mathcal{B}_{i}$ are usually called fractional branes.

What can this decomposition mean for mirror symmetry or geometric Langlands duality? For $r^{*}$ a smooth point, the brane $\mathcal{B}^{*}$ is irreducible and is mapped by duality to an $A$-brane $\mathcal{A}^{*}$ supported on the appropriate fiber $\boldsymbol{F}^{*}$ of the Hitchin fibration of $G . \boldsymbol{F}^{*}$ is irreducible as a Lagrangian submanifold, so the corresponding brane is irreducible as an $A$-brane. When $r^{*}$ is set to $r$, the brane $\mathcal{B}^{*}$ decomposes as a sum of $B$-branes, so the dual $A$-brane $\mathcal{A}^{*}$ must also decompose as a sum of $A$-branes,

$$
\mathcal{A}^{*} \rightarrow \bigoplus_{i} n_{i} \mathcal{A}_{i} \text {. }
$$

It is attractive if this decomposition occurs geometrically. (In fact, we do not know of any other way that it might occur.) In Sections 3 and 5, we will show in detail how a geometrical decomposition occurs for ${ }^{\mathrm{L}} \mathrm{G}=\mathrm{SO}_{3}$. In this example, $\mathcal{M}_{\mathrm{H}}\left(C ;{ }^{\mathrm{L}} G\right)$ contains $A_{1}$ singularities, corresponding to local systems with automorphism group $\mathbb{Z}_{2}$. (These are the simplest examples of endoscopic local systems.) Let $r$ be one of those singularities. Up to isomorphism, there are two irreducible branes supported at $r$, say $\mathcal{B}_{+}$and $\mathcal{B}_{-}$, corresponding to one-dimensional representations of $\mathbb{Z}_{2}$ in which the nontrivial element acts by +1 or -1 . The decomposition (2.3) reads

$$
\mathcal{B}^{*} \rightarrow \mathcal{B}_{+} \oplus \mathcal{B}_{-}
$$

Correspondingly, the relevant Hitchin fiber for $G=S L_{2}$ should decompose as a sum of two components.

We will see this explicitly for $G=S L_{2}$ in Section 3 in genus 1 and in Section 5 in higher genus. Above just those points in the base $\boldsymbol{B}$ of the Hitchin fibration at which the ${ }^{\mathrm{L}} \mathrm{G}=\mathrm{SO}_{3}$ moduli space contains a singularity, the fiber of the Hitchin fibration for $G=S L_{2}$ decomposes as a union of two components $\boldsymbol{F}_{1}$ and $\boldsymbol{F}_{2}$, meeting at two double points. In the example studied in Section 3 (genus 1 with one ramification point), $\boldsymbol{F}_{1}$ and $\boldsymbol{F}_{2}$ are both isomorphic to $\mathbb{C P}^{1}$. They are smooth and are smoothly embedded in 

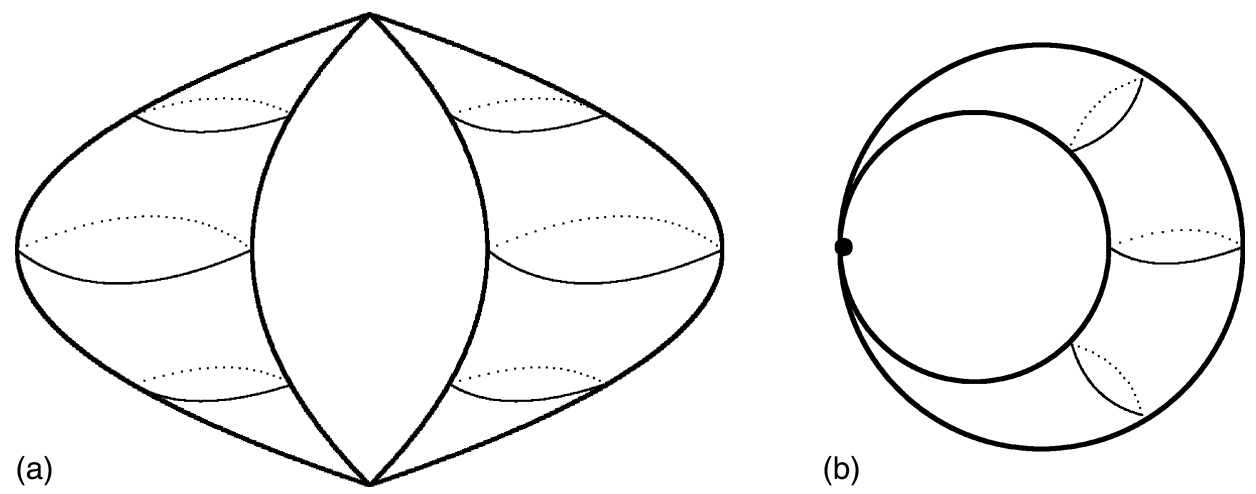

(b)

Figure 1: (a) Singular Hitchin fiber in the $A$-model, $G=S L_{2}$. (b) Singular Hitchin fiber in the $\mathrm{B}$-model, ${ }^{\mathrm{L}} \mathrm{G}=\mathrm{SO}_{3}$.

$\mathcal{M}_{\mathrm{H}}(C ; G)$ and are each $A$-branes in their own right. Our proposal is that the two fractional branes $\mathcal{B}_{+}$and $\mathcal{B}_{-}$supported at the orbifold singularity $r \in \mathcal{M}_{\mathrm{H}}\left(C ;{ }^{\mathrm{L}} G\right)$ map under duality to the $A$-branes $\mathcal{A}_{1}$ and $\mathcal{A}_{2}$ supported on $\boldsymbol{F}_{1}$ and $\boldsymbol{F}_{2}$. (Which of $\mathcal{B}_{+}$and $\mathcal{B}_{-}$maps to $\mathcal{A}_{1}$ and which to $\mathcal{A}_{2}$ is a slightly subtle question that will be discussed in Section 10.)

Though the detailed analysis in this paper will be limited to ${ }^{\mathrm{L}} \mathrm{G}=\mathrm{SO}_{3}$, $G=S L_{2}$, our conjecture is that a similar geometric description of endoscopy holds for all groups (see Section 9).

\subsection{From $A$-branes to $\mathcal{D}$-modules}

What we have discussed so far are $A$-branes on $\mathcal{M}_{\mathrm{H}}(C ; G)$ that are dual to $B$-branes on $\mathcal{M}_{\mathrm{H}}\left(C ;{ }^{\mathrm{L}} G\right)$. However, the geometric Langlands dual of a $B$-brane is usually described not as an $A$-brane but as a twisted $\mathcal{D}$-module on $\mathcal{M}$, the moduli space of stable $G$-bundles on the curve $C$ (and, more generally, on $\operatorname{Bun}_{G}$, the moduli stack of $G$-bundles on $C$ ).

The two viewpoints were reconciled in Section 11 of [39]. The most familiar $A$-branes are branes supported on a Lagrangian submanifold (such as a fiber of the Hitchin fibration), endowed with a flat unitary connection. However, the $A$-model on a symplectic manifold $X$ may in general [38] have additional branes, supported on coisotropic submanifolds whose dimension may exceed half the dimension of $X$. In particular, let $X=T^{*} Y$ be the cotangent bundle of a complex manifold $Y$, with the natural holomorphic two-form $\Omega$. Consider the $A$-model of $X$ with symplectic form $\omega=\operatorname{Im} \Omega$. The $A$-model admits a special brane, the canonical coisotropic $A$-brane $\mathcal{A}_{\text {cc }}$, whose support is all of $X$ and whose existence bridges the gap between 
$A$-branes and $\mathcal{D}$-modules. The endomorphisms of $\mathcal{A}_{\mathrm{cc}}$ (in physical terms, the $\mathcal{A}_{\mathrm{cc}}-\mathcal{A}_{\mathrm{cc}}$ strings) can be sheafified along $Y$ to give a sheaf of rings. This sheaf of rings is the sheaf of differential operators acting on $K_{Y}^{1 / 2}$, where $K_{Y}$ is the canonical bundle of $Y$. We write $\mathcal{D}^{*}$ for the sheaf of such differential operators, and refer to a sheaf of modules for this sheaf of rings (or its generalization introduced below) as a twisted $\mathcal{D}$-module. Now if $\mathcal{A}$ is any other $A$-brane, then $\operatorname{Hom}\left(\mathcal{A}_{\mathrm{cc}}, \mathcal{A}\right)$ can be sheafified along $\mathcal{M}$ to give a twisted $\mathcal{D}$-module. The association $\mathcal{A} \rightarrow \operatorname{Hom}\left(\mathcal{A}_{\mathrm{cc}}, \mathcal{A}\right)$ gives a functor from the category of $A$-branes to the category of twisted $\mathcal{D}$-modules.

To apply this to $A$-branes on $\mathcal{M}_{\mathrm{H}}$, we note that although $\mathcal{M}_{\mathrm{H}}$ is not quite a cotangent bundle, it has a Zariski open set that can be identified with $T^{*} \mathcal{M}$, where $\mathcal{M}$ is the moduli space of stable bundles. An $A$-brane $\mathcal{A}$ on $\mathcal{M}_{\mathrm{H}}$ can be restricted to $T^{*} \mathcal{M}$, and this restriction is non-empty for dimensional reasons. Then we can apply the above construction and associate to $\mathcal{A}$ a twisted $\mathcal{D}$-module on $\mathcal{M}$.

This construction has an analog in which $X$ is not the cotangent bundle of $Y$ but an affine symplectic deformation of one. This means that $X \rightarrow Y$ is a bundle of affine spaces, with a holomorphic symplectic form $\Omega$, such that locally along $Y, X$ is equivalent to $T^{*} Y$. Such an $X$ is obtained by twisting $T^{*} Y$ by an element ${ }^{13} \chi \in H^{1}\left(Y, \Omega^{1, \mathrm{cl}}\right)$, where $\Omega^{1, \mathrm{cl}}$ is the sheaf of closed one-forms on $Y$. In this situation, one can still define ${ }^{14}$ the canonical coisotropic brane $\mathcal{A}_{\mathrm{cc}}$, and the endomorphisms of $\mathcal{A}_{\mathrm{cc}}$ can still be sheafified along $Y$. The sheaf of rings we get is now the sheaf of differential operators acting on $K_{Y}^{1 / 2} \otimes \mathcal{L}$, where ${ }^{15} \mathcal{L}$ is a "line bundle" with $c_{1}(\mathcal{L})=\chi$. Hence now we get a functor from $A$-branes on $Y$ to modules for $\mathcal{D}^{*}(\mathcal{L})$, the sheaf of differential operators acting on $K_{Y}^{1 / 2} \otimes \mathcal{L}$.

This construction was applied in [39] to what mathematically is known as quantum geometric Langlands. (Here it is necessary to consider $X=\mathcal{M}_{\mathrm{H}}$ in a complex structure obtained by a hyper-Kahler rotation of $I$.) More

${ }^{13}$ To construct $X$, one pulls back $\chi$ to $H^{1}\left(T^{*} Y, \Omega^{1, \mathrm{cl}}\left(T^{*} Y\right)\right)$ and uses the symplectic form of $T^{*} Y$ to map this pullback to $H^{1}\left(T^{*} Y, T\left(T^{*} Y\right)\right)$, which classifies deformations of $T^{*} Y$. The resulting deformation is symplectic because we start with $\Omega^{1, \mathrm{cl}}$.

${ }^{14}$ It is necessary to require that the cohomology class of $\operatorname{Re} \chi$ is equal to a quantum parameter called $\eta$ in [30]. Except in Section 4.7, we emphasize the classical picture and suppress the role of $\eta$.

${ }^{15}$ In general, the cohomology class of $\chi$ is not integral, so $\mathcal{L}$ may be the complex power of a line bundle (or a tensor product of such) rather than an ordinary complex line bundle. But the sheaf of differential operators acting on $\mathcal{L}$, or on $K_{Y}^{1 / 2} \otimes \mathcal{L}$, still makes sense. 
relevant for our purposes, it was applied in [30] to the ramified case of geometric Langlands. Here one uses the fact that in the ramified case, a Zariski open set in $\mathcal{M}_{\mathrm{H}}$ can be identified with an affine symplectic deformation of $T^{*} \mathcal{M}$. (This is described in detail for our example in Section 3.9.) For the ramified case of geometric Langlands, this construction leads to the following statement: there is a natural functor from $A$-branes of $\mathcal{M}_{\mathrm{H}}(C ; G)$ to $\mathcal{D}^{*}(\mathcal{L})$-modules on $\mathcal{M}$, where $\mathcal{M}$ is now the moduli space of stable parabolic $G$-bundles on $C$ and the first Chern class of $\mathcal{L}$ is the logarithm of the monodromy of the dual ${ }^{\mathrm{L}} G$ local system.

2.3.1. Relation to a local system Now let us discuss how the twisted $\mathcal{D}$-modules arising from $A$-branes in this situation may be related to local systems. We let $X$ be $T^{*} Y$ or an affine deformation thereof, and let $\pi: X \rightarrow Y$ be the projection. Let $L$ be a compact (complex) Lagrangian submanifold of $X$ such that the map $\pi: L \rightarrow Y$ is an $n$-fold cover. Then the functor from $A$-branes to twisted $\mathcal{D}$-modules is expected to map a rank $1 A$-brane supported on $L$ to a local system on $Y$ of rank $n$ (twisted by $K_{Y}^{1 / 2}$ ), or in other words to a rank $n$ complex vector bundle $V \rightarrow Y$ with a flat connection (or a connection of central curvature in case of an affine deformation).

This has an important generalization if $L$ is closed in $X$ but not compact, and the map $\pi: L \rightarrow Y$ is generically $n$ to 1 , but is of lower degree on a divisor. This happens if, intuitively, some branches of $L$ go to infinity over the divisor in question. For simplicity, suppose that $Y$ is a curve, as is actually the case in the example of Section 3. Let $u$ be a local parameter on $Y$, and let $s$ be a function linear on the fibers of $X \rightarrow Y$ such that locally along $Y$ the symplectic form of $X$ is $\Omega=d u \wedge d s$. Then $L$ can be described locally by an $n$-valued function $s(u)$ and the situation that we are interested in is that some branches of this function are singular at a point $r \in Y$ corresponding to, say, $u=u_{0}$. We assume that the $n$ branches look like

$$
s_{i}(u) \sim c_{i}\left(u-u_{0}\right)^{-d_{i}}, \quad i=1, \ldots, n .
$$

(The $d_{i}$ are not necessarily integers, since the map $\pi: L \rightarrow Y$ may be ramified at $u=u_{0}$.) In this situation, an $A$-brane supported on $L$ will map to a twisted $\mathcal{D}$-module on $Y$ that is represented by a local system $V \rightarrow Y \backslash\{r\}$ with a singularity at $r$. The nature of the singularity is largely determined by the $d_{i}$ and $c_{i}$. For example, the condition for a regular singularity is that $d_{i} \leq 1$, and the monodromies at a regular singularity are then largely determined by the $c_{i}$. For this reason, we compute the $c_{i}$ and $d_{i}$ for our 
example in Equation (3.49). In Section 4, we explain how these coefficients are expected to be related to the singularities of the local system. We also describe the generalization to higher dimensions.

2.3.2. Eigenbranes and eigensheaves Mirror symmetry of $\mathcal{M}_{\mathrm{H}}$ has many special properties related to its origin in four dimensions. For example, the Wilson and 't Hooft line operators of four-dimensional gauge theory can be reinterpreted in two-dimensional terms [39] and are essential for understanding the Hecke operators of the geometric Langlands program.

The correspondence from $A$-branes to $\mathcal{D}$-modules should map an $A$-brane which is an eigenbrane of the 't Hooft operators to a $\mathcal{D}$-module on $\mathcal{M}$ (and more generally, on $\operatorname{Bun}_{G}$, the moduli stack of $G$-bundles on our curve $C$ ) which is a Hecke eigensheaf. These Hecke eigensheaves are the main objects of interest in the geometric Langlands correspondence (in its usual formulation). The geometric Langlands conjecture predicts that to each ${ }^{\mathrm{L}} G$-local system $\mathcal{E}$ on $C$, one may associate a category $\mathcal{A} \mathrm{ut}_{E}$ of Hecke eigensheaves on $\operatorname{Bun}_{G}$. In particular, if the group of automorphisms of the local system is trivial, then it is expected that this category is equivalent to the category of vector spaces. In other words, it contains a unique irreducible object, and all other objects are direct sums of copies of this object. The challenge is to describe what happens for local systems with nontrivial groups of automorphisms.

However, this is rather difficult to do using the language of $\mathcal{D}$-modules. In those cases in which Hecke eigensheaves have been constructed explicitly (for instance, for $G=S L_{n}$ ), their structure is notoriously complicated. This makes it difficult to extract useful information. The language of $A$-branes, on the other hand, is much better adapted to analyzing the structure of the corresponding categories of eigenbranes. As discussed above, the generic eigenbranes are unitary flat local systems on the smooth Hitchin fibers, and the special eigenbranes associated with endoscopy are supported on the singular Hitchin fibers. It turns out that one can describe these singular fibers, and hence the corresponding eigenbranes, very explicitly. For instance, we observe that the eigenbranes supported on the singular fibers break into pieces, the "fractional branes" discussed earlier. Furthermore, these fractional branes satisfy a certain modification of the eigenbrane property discussed in Section 5.3 (the fractional eigenbrane property).

We then translate these results to the language of $\mathcal{D}$-modules. Thus, if an eigenbrane $\mathcal{A}$ decomposes into two irreducible branes $\mathcal{A}_{1}$ and $\mathcal{A}_{2}$, then it is natural to predict that the corresponding Hecke eigensheaf $\mathcal{F}$ will also decompose as a direct sum of two $\mathcal{D}$-modules, $\mathcal{F}_{1}$ and $\mathcal{F}_{2}$, corresponding to 
$\mathcal{A}_{1}$ and $\mathcal{A}_{2}$, respectively. Furthermore, these two $\mathcal{D}$-modules should then satisfy a fractional Hecke eigensheaf property described in Section 6 .

The upshot of all this is that by analyzing the categories of $A$-branes supported on the singular Hitchin fibers, we gain a lot of insight into the geometric Langlands correspondence, and, hopefully, even into the classical Langlands correspondence for curves over finite fields. We will describe this in detail in explicit examples presented below.

\section{Explicit example in genus 1}

\subsection{Higgs bundles in genus 1}

To construct an explicit example in which we can see the geometric analog of endoscopy, we take $G=S L_{2}$, and we work on a Riemann surface $C$ of genus $g_{C}=1$, with a single point $p$ of ramification. One might think that ramification would bring an extra complication, but actually, the case of genus 1 with a single ramification point is particularly simple and has often been considered in the literature on Hitchin fibrations. For $g_{C} \geq 2$, the relevant moduli spaces have higher dimension and explicit computation is difficult; for $g_{C} \leq 1$, the fundamental group of $C$ is abelian (or trivial), which in the absence of ramification leads to complications, unrelated to endoscopy, that we prefer to avoid here.

As we explain in Section 5.3, for $g_{C}>1$, though explicit computation is difficult, the method of spectral curves is a powerful substitute. But we prefer to begin with the case of $g_{C}=1$ for which everything can be computed directly.

First we describe Higgs bundles without ramification on a Riemann surface $C$ of genus 1 . We begin with ordinary $S L_{2}$ Higgs bundles, that is, pairs $(E, \varphi)$ where $E$ is a rank 2 bundle of trivial determinant. If $E$ is semi-stable, it must have the form

$$
E=\mathcal{L} \oplus \mathcal{L}^{-1}
$$

where $\mathcal{L}$ is a complex line bundle of degree 0 . If $\mathcal{L}$ is nontrivial, $\phi$ must be in this basis

$$
\phi=\left(\begin{array}{cc}
a & 0 \\
0 & -a
\end{array}\right),
$$

where $a$ is an ordinary holomorphic differential on $C$. The choice of $\mathcal{L}$ is parametrized by a curve $C^{\prime}$ (the Jacobian of $C$ ) that is isomorphic to $C$, 
and the space of holomorphic differentials is one-dimensional. So we have constructed a family of semi-stable Higgs bundles parametrized by $C^{\prime} \times \mathbb{C}$, where the choice of $\mathcal{L}$ gives a point in $C^{\prime}$ and the choice of $a$ gives a point in $\mathbb{C}$. (We have implicitly picked a particular holomorphic differential on $C$ to identify the space of such differentials with $\mathbb{C}$.) However, replacing $\mathcal{L}$ by $\mathcal{L}^{-1}$ and changing the sign of $a$ gives back the same Higgs bundle, up to isomorphism. This operation can be understood as a gauge transformation

$$
\left(\begin{array}{cc}
0 & 1 \\
-1 & 0
\end{array}\right)
$$

So we can take the quotient by $\mathbb{Z}_{2}$ and we get a family of semi-stable Higgs bundles parametrized by $\left(C^{\prime} \times \mathbb{C}\right) / \mathbb{Z}_{2}$. This actually is the moduli space of rank 2 semi-stable Higgs bundles over $C$ of trivial determinant.

\subsection{Ramification}

Now let us incorporate ramification. In the context of Higgs bundles, ramification means [68] that $\varphi$ may have a pole at a prescribed point $p \in C$ (or more generally at several such points) and with a prescribed characteristic polynomial of the polar part. We will consider the case of a simple pole. In addition, in the fiber $E_{p}$ of the bundle $E$ at $p$, one is given a $\varphi$-invariant parabolic structure, that is, a flag that is invariant under the action of the polar part of $\varphi$. This flag, moreover, is endowed with parabolic weights. The whole structure can be described uniformly by adapting Hitchin's equations to incorporate singularities. The behavior near $p$ is determined by parameters $\alpha, \beta$ and $\gamma$ valued in the Lie algebra $\mathfrak{t}$ of a maximal torus of the compact form of $G$. Let $z$ be a local parameter near $p$ and write $z=r e^{i \theta}$. In the notation of [30], the local behavior near $p$ is

$$
\begin{aligned}
& A=\alpha d \theta+\cdots \\
& \phi=\beta \frac{d r}{r}-\gamma d \theta+\cdots .
\end{aligned}
$$

Since the Higgs field $\varphi$ is simply the $(1,0)$ part of $\phi$, these equations immediately determine its polar behavior, up to gauge-equivalence or conjugacy. One has $\varphi \sim \sigma d z / z$, where $\sigma=(\beta+i \gamma) / 2$. The $\varphi$-invariant parabolic structure is completely determined by $\varphi$ and a choice of Borel subgroup containing $\sigma$. If $\sigma$ is regular, as we will generally assume, there are only finitely many choices of Borel subgroup containing $\sigma$, and a choice can be made by ordering the eigenvalues of $\sigma$. Consequently, for studying ramified Higgs 
bundles with regular $\sigma$, the parabolic structure (or the choice of $\alpha$ ) need not be specified explicitly.

Similarly, we can determine the monodromy around $p$ of the local system with connection $\mathcal{A}=A+i \phi$. It is

$$
M=\exp (-2 \pi(\alpha-i \gamma))
$$

Now let us specialize to the case of a genus 1 curve $C$ with one ramification point. It is convenient to describe $C$ explicitly by an algebraic equation

$$
y^{2}=f(x)
$$

where we can take $f$ to be of the form

$$
f(x)=x^{3}+a x+b
$$

and interpret $p$ as the point at infinity. We assume that $f$ has distinct roots, so that $C$ is smooth.

To explicitly describe the ramified Higgs bundles $(E, \varphi)$, we first, as before, take $E=\mathcal{L} \oplus \mathcal{L}^{-1}$, where $\mathcal{L}$ is of degree zero. We can take $\mathcal{L}=$ $\mathcal{O}(p) \otimes \mathcal{O}(q)^{-1}$, where $q$ is some point in $C$, corresponding to $(x, y)=\left(x_{0}, y_{0}\right)$. A Higgs field will be of the form

$$
\varphi=\left(\begin{array}{cc}
h & k \\
g & -h
\end{array}\right),
$$

where $h$ is a section of $K \otimes \mathcal{O}(p), k$ is a section of $K \otimes \mathcal{O}(p) \otimes \mathcal{L}^{2}$, and $g$ is a section of $K \otimes \mathcal{O}(p) \otimes \mathcal{L}^{-2}$. Of course, for $C$ of genus $1, K$ is trivialized by the differential $d x / y$. The relevant line bundles all have one-dimensional spaces of holomorphic sections, and the general forms for $k, g$, and $h$ are

$$
\begin{aligned}
& h=\frac{d x}{y} h_{0} \\
& k=\frac{d x}{y} k_{0}\left(\left(y-y_{0}\right)-\frac{f^{\prime}\left(x_{0}\right)\left(x-x_{0}\right)}{2 y_{0}}\right) \\
& g=\frac{d x}{y} g_{0}\left(\left(y+y_{0}\right)+\frac{f^{\prime}\left(x_{0}\right)\left(x-x_{0}\right)}{2 y_{0}}\right) \frac{1}{\left(x-x_{0}\right)^{2}} .
\end{aligned}
$$

where $h_{0}, k_{0}$, and $g_{0}$ are complex constants, and the formulas were obtained as follows. The formula for $h$ requires no explanation. After trivializing $K$, $k$ is supposed to be a holomorphic section of $\mathcal{O}(p) \otimes \mathcal{L}^{2}=\mathcal{O}(p)^{3} \otimes \mathcal{O}(q)^{-2}$, 
so we have looked for a function that is holomorphic except for a triple pole at infinity, and moreover has a double zero at $q$. Such a function is $\left(y-y_{0}\right)-f^{\prime}\left(x_{0}\right)\left(x-x_{0}\right) / 2 y_{0}$. Similarly, $g$ is supposed to be a holomorphic section of $\mathcal{O}(p) \otimes \mathcal{L}^{-2}=\mathcal{O}(p)^{-1} \otimes \mathcal{O}(q)^{2}$, so we have looked for a function that is holomorphic except for a double pole at $q$ and vanishes at infinity. Such a function is $\left(\left(y+y_{0}\right)+f^{\prime}\left(x_{0}\right)\left(x-x_{0}\right) / 2 y_{0}\right) /\left(x-x_{0}\right)^{2}$.

The next step is to evaluate the characteristic polynomial of $\varphi$. For $G=S L_{2}$, this simply means that we should compute $\operatorname{Tr} \varphi^{2}$, which in the present case turns out to be

$$
\operatorname{Tr} \varphi^{2}=\left(\frac{d x}{y}\right)^{2}\left(2 h_{0}^{2}+2 k_{0} g_{0}\left(x+2 x_{0}-\frac{f^{\prime}\left(x_{0}\right)^{2}}{4 f\left(x_{0}\right)}\right)\right) .
$$

The polar part of $\operatorname{Tr} \varphi^{2}$ is simply $(d x / y)^{2} 2 x k_{0} g_{0}$, which has a double pole at infinity. If $z$ is a local parameter at infinity, we have $x \sim z^{-2}, y \sim z^{-3}$, and $(d x / y)^{2} x \sim 4(d z / z)^{2}$. The polar part of $\varphi$ is supposed to be conjugate to $\sigma d z / z$, so we want $\operatorname{Tr} \varphi^{2} \sim \operatorname{Tr} \sigma^{2}(d z / z)^{2}=2 \sigma_{0}^{2}(d z / z)^{2}$, where we denote the eigenvalues of $\sigma$ as $\pm \sigma_{0}$. So we set $k_{0} g_{0}=\sigma_{0}^{2} / 4$, and write

$$
\operatorname{Tr} \varphi^{2}=\left(\frac{d x}{y}\right)^{2}\left(2 h_{0}^{2}+\frac{\sigma_{0}^{2}}{2}\left(x+2 x_{0}-\frac{f^{\prime}\left(x_{0}\right)^{2}}{4 f\left(x_{0}\right)}\right)\right) .
$$

The reason that only the product $k_{0} g_{0}$ is determined is that the bundle $E=\mathcal{L} \oplus \mathcal{L}^{-1}$ has an automorphism group $\mathbb{C}^{\times}$, acting on the two summands as multiplication by $\lambda$ and $\lambda^{-1}$, respectively, and transforming $k_{0}$ and $g_{0}$ by $k_{0} \rightarrow \lambda^{2} k_{0}, g_{0} \rightarrow \lambda^{-2} g_{0}$.

Formula (3.9) breaks down if $y_{0}=0$ (because $y_{0}$ appears in the denominator in the formulas for $k$ and $g$ ) or $y_{0}=\infty$ (since the formulas for $k$ and $g$ also contain terms linear in $y_{0}$ ), or equivalently if $\mathcal{L}$ is of order 2 . This happens because when $\mathcal{L}$ is of order 2 , we have $\mathcal{L} \cong \mathcal{L}^{-1}$ and (if $\sigma \neq 0$ ) there does not exist a stable or semi-stable ramified Higgs bundle $(E, \varphi)$ with $E=\mathcal{L} \oplus \mathcal{L}^{-1}=\mathcal{L} \oplus \mathcal{L}$. Indeed, with that choice of $E$, a holomorphic section of $K \otimes \operatorname{ad}(E) \otimes \mathcal{O}(p) \cong K \otimes \mathcal{O}(p)^{\oplus 3}$ cannot have a pole at $p$, and hence the condition $\operatorname{Tr} \varphi^{2} \sim 2 \sigma_{0}^{2}(d z / z)^{2}$ cannot be obeyed. Instead, if $\mathcal{L}$ is of order 2, and $\sigma \neq 0$, one must take $E$ to be a nontrivial extension of $\mathcal{L}$ by $\mathcal{L}$.

\subsection{The moduli space}

For each choice of the parameter $\sigma_{0}^{2}$, we have constructed a family of ramified Higgs bundles. The underlying bundle is $E=\mathcal{L} \oplus \mathcal{L}^{-1}$, and the Higgs field $\varphi$ has been described above. The choice of $E$ depends on the point $q$ or 
equivalently the pair $\left(x_{0}, y_{0}\right)$ (with $y_{0}^{2}=f\left(x_{0}\right)$ ), and the choice of $\varphi$ depends additionally on the parameter $h_{0}$. To construct in this situation the moduli space $\mathcal{M}_{\mathrm{H}}$ of ramified Higgs bundles, we must take account of the exchange $\tau: \mathcal{L} \rightarrow \mathcal{L}^{-1}$, which acts by $\left(x_{0}, y_{0}, h_{0}\right) \rightarrow\left(x_{0},-y_{0},-h_{0}\right)$.

The pair $\left(q, h_{0}\right)$ or triple $\left(x_{0}, y_{0}, h_{0}\right)$ defines a point in $C \times \mathbb{C}$, and after allowing for the symmetry $\tau$, it seems that the moduli space is $(C \times \mathbb{C}) / \mathbb{Z}_{2}$, independent of $\sigma_{0}$. This is actually not quite correct, because of the point made at the end of Section 3.2. The space $(C \times \mathbb{C}) / \mathbb{Z}_{2}$ has four $A_{1}$ singularities, at points with $h_{0}=0$ and $q$ of order 2 . For $\sigma \neq 0$, the $A_{1}$ singularities are deformed and the moduli space becomes smooth. We have not seen the deformation because our analysis does not cover the case that $q$ is of order 2 . If $\sigma=0$ and $\alpha \neq 0$, the $A_{1}$ singularities are resolved rather than deformed; from a hyper-Kahler point of view, the phenomenon, for generic values of $\sigma$ and $\alpha$, is really a simultaneous deformation and resolution, as in [41]. In this paper, we will not describe this deformation or resolution directly, but in Equation (3.15), the deformed moduli space is described, with the aid of the Hitchin fibration.

Perhaps we should make a comment here on the role of ramification in genus 1. For $g_{C}>1$, a generic semi-stable bundle is actually stable, but for $g_{C}=1$ (and no ramification) and simply connected $G$, there are no strictly stable bundles. The closest one can come is a semi-stable bundle, such as $E=\mathcal{L} \oplus \mathcal{L}^{-1}$ for $G=S L_{2}$. Likewise, in general, $\mathcal{M}_{\mathrm{H}}$ parametrizes stable and semi-stable Higgs bundles. For $g_{C}=1$, unramified Higgs bundles are at best semi-stable, so $\mathcal{M}_{\mathrm{H}}$ actually parametrizes semi-stable Higgs bundles. The situation changes with ramification. Semi-stable Higgs bundles with one ramification point are actually stable (if $\sigma \neq 0$ ). The reason for this is that the potential destabilizing sheaves of $E=\mathcal{L} \oplus \mathcal{L}^{-1}$ (namely the summands $\mathcal{L}$ and $\mathcal{L}^{-1}$ ) are not $\varphi$-invariant, and so do not contradict stability of the Higgs bundle $(E, \varphi)$.

\subsection{The Hitchin fibration}

Next we need to understand the Hitchin fibration. The Hitchin fibration is simply the map that takes a Higgs bundle $(E, \varphi)$ to the characteristic polynomial of $\varphi$. For $G=S L_{2}$, this characteristic polynomial reduces to $\operatorname{Tr} \varphi^{2}$. In the present context, according to (3.11), that characteristic polynomial is a polynomial in $x$ of degree 1 (times $\left.(d x / y)^{2}\right)$. The coefficient of the linear term in $x$ is fixed, and the Hitchin fibration is the map that extracts the constant term, which we will call $w_{0}$. In other words, the Hitchin fibration 
maps the Higgs bundle $(E, \varphi)$ to

$$
w_{0}=2 h_{0}^{2}+\frac{\sigma_{0}^{2}}{2}\left(2 x_{0}-\frac{f^{\prime}\left(x_{0}\right)^{2}}{4 f\left(x_{0}\right)}\right) .
$$

The fiber of the Hitchin fibration is described by variables $x_{0}, y_{0}$ and $h_{0}$ obeying (3.12) and

$$
y_{0}^{2}=f\left(x_{0}\right)
$$

and subject also to the $\mathbb{Z}_{2}$ symmetry $\left(x_{0}, y_{0}, h_{0}\right) \rightarrow\left(x_{0},-y_{0},-h_{0}\right)$. Apart from $x_{0}$, the $\mathbb{Z}_{2}$ invariants are $y_{0}^{2}, h_{0}^{2}$ and

$$
\rho=\left(\frac{2}{\sigma_{0}}\right) y_{0} h_{0} .
$$

(The factor of $2 / \sigma_{0}$ has been included for convenience.) Of these, $y_{0}^{2}$ and $h_{0}^{2}$ are equivalent to rational functions of $x_{0}$ according to the last two equations, and so can be omitted, while $\rho$ obeys a quadratic equation. If henceforth we write $u$ for $x_{0}$, and set $w_{0}=-w \sigma_{0}^{2} / 2$, then the equation obeyed by $\rho$ is

$$
\rho^{2}=-(2 u+w) f(u)+\frac{f^{\prime}(u)^{2}}{4} .
$$

This equation for complex variables $\rho, u$ and $w$ describes a complex surface which is the moduli space $\mathcal{M}_{\mathrm{H}}$ of ramified $S L_{2}$ Higgs bundles. (Some points at $u, \rho=\infty$ are omitted in this way of writing the equation.) There is a simple explanation for why it does not depend on the parameters $\alpha, \beta$ and $\gamma$ that characterize ramification. Complex structure $I$ does not depend on $\alpha$, and as long as there is only one ramification point, $\sigma=(\beta+i \gamma) / 2$ can be eliminated by rescaling $\varphi$ (provided it is not zero), as we have done.

If we set $w$ to a fixed complex number, we get an algebraic curve $\boldsymbol{F}_{w}$ which is the fiber of the Hitchin fibration. The right-hand side is a quartic polynomial in $u$ and, for generic $w, \boldsymbol{F}_{w}$ is a smooth curve of genus 1 .

When is $\boldsymbol{F}_{w}$ singular? This occurs precisely if two roots of the polynomial $g(u)=(2 u+w) f(u)-f^{\prime}(u)^{2} / 4$ coincide, or in other words if its discriminant vanishes. Let $e_{1}, e_{2}$, and $e_{3}$ be the roots of the polynomial $f(u)=u^{3}+a u+b$. (Of course, $e_{1}+e_{2}+e_{3}=0$, and we assume that the $e_{i}$ are distinct so that $C$ is smooth.) The discriminant of $g$ is $\left(e_{1}-e_{2}\right)^{2}$ $\left(e_{2}-e_{3}\right)^{2}\left(e_{3}-e_{1}\right)^{2}\left(w-e_{1}\right)^{2}\left(w-e_{2}\right)^{2}\left(w-e_{3}\right)^{2}$, so $\boldsymbol{F}_{w}$ is singular precisely 
if $w$ is equal to one of the $e_{i}$. For example, if $w=e_{1}$, we find that

$$
-(2 u+w) f(u)+\frac{f^{\prime}(u)^{2}}{4}=\frac{1}{4}\left(\left(u-e_{1}\right)^{2}-\left(e_{1}-e_{2}\right)\left(e_{1}-e_{3}\right)\right)^{2} .
$$

Of course, there are similar formulas if $w=e_{2}$ or $w=e_{3}$. The fact that the left-hand side of (3.16) is a perfect square means that at $w=e_{1}$, the curve $\boldsymbol{F}_{w}$ splits as a union of two components $\boldsymbol{F}_{e_{1}}^{ \pm}$defined by

$$
\rho= \pm \frac{1}{2}\left(\left(u-e_{1}\right)^{2}-\left(e_{1}-e_{2}\right)\left(e_{1}-e_{3}\right)\right)
$$

The curves $\boldsymbol{F}_{e_{1}}^{ \pm}$are each of genus 0 . They meet at the two points given by

$$
\rho=0, \quad u=e_{1} \pm \sqrt{\left(e_{1}-e_{2}\right)\left(e_{1}-e_{3}\right)} .
$$

A pair of genus 0 curves meeting at two double points gives a curve of arithmetic genus 1. A smooth curve of genus 1 can degenerate to such a singular curve. This is the behavior of the Hitchin fibration in our example at the three fibers $w=e_{1}, e_{2}$ and $e_{3}$. These singular fibers are shown in Fig. 1a. The fact that there are two double points when $w$ equals one of the $e_{i}$ is the reason that the discriminant of $g$ has a double zero at those values of $w$.

Although the fiber $\boldsymbol{F}_{w}$ of the Hitchin fibration is singular when $w=e_{i}$, the moduli space $\mathcal{M}_{\mathrm{H}}$ of stable ramified $S L_{2}$ Higgs bundles over $C$ is actually smooth. Indeed, since $f \neq 0$ at the points $\rho=0, w=e_{i}$, the polynomial $\rho^{2}+$ $(2 u+w) f(u)-f^{\prime}(u)^{2} / 4$ whose vanishing characterizes $\mathcal{M}_{\mathrm{H}}$ has a nonzero differential at those points. So $\mathcal{M}_{\mathrm{H}}\left(C ; S L_{2}\right)$ is smooth even though the fibers of the Hitchin fibration are singular.

What we have found is precisely the behavior that was promised in Section 2.2. Certain fibers of the Hitchin fibration split up as the union of two components $\boldsymbol{F}^{ \pm}$, leading to a decomposition of $A$-branes. In Section 3.6, we explain how this is related to singularities of the moduli space for the dual group.

\subsection{Symmetry group}

An $S L_{2}$ Higgs bundle $(E, \varphi)$ can be twisted by a line bundle $\mathcal{N}$ of order 2 . To be more precise, this operation is $E \rightarrow E \otimes \mathcal{N}, \varphi \rightarrow \varphi$. For $C$ of genus 1 , the group of line bundles of order 2 is $Q=\mathbb{Z}_{2} \times \mathbb{Z}_{2}$, and this group must act on the moduli space $\mathcal{M}_{\mathrm{H}}$ of ramified stable Higgs bundles. 
The underlying bundle $E$ is $E=\mathcal{L} \oplus \mathcal{L}^{-1}$, where modulo the exchange $\mathcal{L} \leftrightarrow \mathcal{L}^{-1}, \mathcal{L}$ is parametrized by $u=x_{0}$. The group $Q$ acts on $E$ by holomorphic automorphisms of the moduli space of semi-stable bundles. Such an automorphism is a fractional linear transformation of the $u$-plane. Moreover, the condition for $\mathcal{L}$ to be of order 2 is invariant under the action of $Q$.

$\mathcal{L}$ is trivial if $u=\infty$ and is a nontrivial line bundle of order 2 if $u=e_{1}, e_{2}$ or $e_{3}$. Twisting with a line bundle of order 2 therefore exchanges $u=\infty$ with one of the values $u=e_{i}$ while also exchanging the other two $e$ 's. For example, there is an element $T_{1} \in Q$ that exchanges $\infty$ with $e_{1}$ and also exchanges $e_{2}$ with $e_{3}$. It acts by

$$
\begin{aligned}
u & \rightarrow \frac{e_{1} u+e_{2} e_{3}-e_{1} e_{3}-e_{1} e_{2}}{u-e_{1}} \\
w & \rightarrow w \\
\rho & \rightarrow-\frac{\left(e_{1}-e_{2}\right)\left(e_{1}-e_{3}\right)}{\left(u-e_{1}\right)^{2}} \rho .
\end{aligned}
$$

The fact that $w$ is invariant reflects the fact that $\varphi$, and therefore its characteristic polynomial, is invariant under twisting by a line bundle of order 2 . The sign in the transformation of $\rho$ is not obvious (Equation (3.15) that defines $\mathcal{M}_{\mathrm{H}}$ is invariant under $\rho \rightarrow-\rho$ ) and can be determined from the fact that the holomorphic two-form $\Omega_{I}$ of $\mathcal{M}_{\mathrm{H}}$ is $Q$-invariant. $Q$ also has elements $T_{2}$ and $T_{3}$ that are obtained by cyclic permutation of $e_{1}, e_{2}$ and $e_{3}$. These are the three nontrivial elements of $Q$.

Since $w$ is $Q$-invariant, the group $Q$ acts on each fiber $\boldsymbol{F}_{w}$ of the Hitchin fibration. Let us describe the action on the singular fiber at, say, $w=e_{1}$. A short calculation with the above formulas shows that $T_{1}$ maps each component $\boldsymbol{F}_{e_{1}}^{ \pm}$to itself and leaves fixed the two double points of Equation (3.18). The curves $\boldsymbol{F}_{e_{1}}^{ \pm}$have genus 0, and $T_{1}$ acts on each of these genus 0 curves as a fractional linear transformation with two fixed points, namely the double points. (For an involution of $\mathbb{C P}^{1}$ with two fixed points, consider the transformation $z \rightarrow-z$ of the complex $z$-plane, with fixed points at 0 and $\infty$.) On the other hand, $T_{2}$ and $T_{3}$ exchange the two components $\boldsymbol{F}_{e_{1}}^{ \pm}$and also exchange the two double points. This being the case, $T_{2}$ and $T_{3}$ act freely on the singular curve $\boldsymbol{F}_{e_{1}}$, and therefore also on all nearby fibers of the Hitchin fibration.

Remark 3.1. $Q$ acts on each fiber $\boldsymbol{F}_{w}$ of the Hitchin fibration by translation by the group of points of order 2 . This is clear in the spectral curve construction that is described in Section 5.3. Hence when $\boldsymbol{F}_{w}$ is smooth, $Q$ acts freely. When $\boldsymbol{F}_{w}$ is degenerating to a union of two components $\boldsymbol{F}_{w}^{ \pm}$, 
it has a "short" direction corresponding to a one-cycle that collapses at a double point and a complementary "long" direction. $T_{2}$ and $T_{3}$ are translations in the long direction; they act freely and exchange the two double points and the two components. $T_{1}$ is a translation in the short direction, maps each component to itself, and has the double points as fixed points.

\subsection{Langlands dual group}

The Langlands dual group of $G=S L_{2}$ is ${ }^{\mathrm{L}} G=S O_{3}$ or equivalently $P G L_{2}$. We would therefore also like to understand Higgs bundles on $C$ with structure group $S_{3}$. These are closely related to $S L_{2}$ Higgs bundles, because $S O_{3}=S L_{2} / \mathbb{Z}_{2}$ is the adjoint form of $S L_{2}$.

We will let $W$ denote a holomorphic $\mathrm{SO}_{3}$ bundle; that is, a rank 3 holomorphic bundle with a nondegenerate holomorphic quadratic form and volume form. Since the three-dimensional representation of $\mathrm{SO}_{3}$ is the adjoint representation, we need not distinguish between $W$ and the corresponding adjoint bundle.

The moduli space of $\mathrm{SO}_{3}$ Higgs bundles $(W, \varphi)$ over a Riemann surface $C$ has two components, distinguished ${ }^{16}$ by the second Stieffel-Whitney class $w_{2}(W)$ of the underlying bundle $W$. We denote these components as $\mathcal{M}_{\mathrm{H}}\left(C ; S O_{3}, w_{2}\right)$, where $w_{2}$ is either 0 or is the nonzero element of $H^{2}\left(C, \mathbb{Z}_{2}\right) \cong \mathbb{Z}_{2}$, which we call $\theta$. Let us first consider the case that $w_{2}(W)=0$.

The structure group of an $\mathrm{SO}_{3}$-bundle $\mathrm{W}$ with $w_{2}=0$ can be lifted to $S L_{2}$, and we can then form an associated rank two bundle $E$, with structure group $S L_{2} . \quad E$ is uniquely determined up to twisting by a line bundle of order 2. Consequently, there is a very simple relation between the moduli space $\mathcal{M}_{\mathrm{H}}\left(C ; S O_{3}, 0\right)$ of $S O(3)$ Higgs bundles with vanishing $w_{2}$ and the corresponding $S L_{2}$ moduli space $\mathcal{M}_{\mathrm{H}}\left(C ; S L_{2}\right)$ :

$$
\mathcal{M}_{\mathrm{H}}\left(C ; S O_{3}, 0\right)=\frac{\mathcal{M}_{\mathrm{H}}\left(C ; S L_{2}\right)}{Q} .
$$

We can immediately use our knowledge of the action of $Q$ to describe the fiber of the Hitchin fibration of $\mathcal{M}_{\mathrm{H}}\left(C ; S_{3}, 0\right)$ at the special points $w=e_{1}, e_{2}, e_{3}$. At, say, $w=e_{1}$, the effect of dividing by $T_{2}$ (or $T_{3}$ ) is to identify the two components $\boldsymbol{F}_{e_{1}}^{ \pm}$. So we can just focus on one of them, say $\boldsymbol{F}_{e_{1}}^{+}$. It is a curve of genus 0 with two points identified (namely the double points that are exchanged by $T_{2}$ ). We still must divide by $T_{1}$; the quotient

\footnotetext{
${ }^{16}$ See Remark 3.3 for a more precise statement.
} 
is again a curve of genus 0 with a double point. So that is the nature of the exceptional fibers of the Hitchin fibration for ${ }^{\mathrm{L}} \mathrm{G}=\mathrm{SO}_{3}$. They are shown in fig. 1b. In particular, the special Hitchin fibers for $\mathrm{SO}_{3}$ are irreducible, while those for $S L_{2}$ have two components.

Now we come to another crucial difference between $\mathrm{SL}_{2}$ and $\mathrm{SO}_{3}$. In the case of $S L_{2}$, though some fibers of the Hitchin fibration are singular, the singularities of the fibers are not singularities of $\mathcal{M}_{\mathrm{H}} ; \mathcal{M}_{\mathrm{H}}$ is smooth near the exceptional fibers (and in fact everywhere, for generic $\sigma$ ).

But dividing $\mathcal{M}_{\mathrm{H}}\left(C ; S L_{2}\right)$ by $Q=\mathbb{Z}_{2} \times \mathbb{Z}_{2}$ creates a singularity. $T_{2}$ acts freely on the fiber $\boldsymbol{F}_{e_{1}}$, and therefore on a small neighborhood of it. But $T_{1}$ leaves fixed the two double points of the fiber. Dividing by $T_{1}$ therefore creates two $A_{1}$ singularities. These are exchanged by the action of $T_{2}$, and therefore the quotient $\mathcal{M}_{\mathrm{H}}\left(C ; S O_{3}, 0\right)$ has one $A_{1}$ singularity on each exceptional fiber.

3.6.1. Relation to geometric endoscopy What we have just described is the picture promised in Section 2.2. For $\mathrm{SO}_{3}$, the special fiber of the Hitchin fibration has an $A_{1}$ singularity at a point $r$. A $B$-brane $\mathcal{B}^{*}$ supported at a generic point $r^{*}$ is irreducible, but for $r^{*}=r$, it can split up as a sum $\mathcal{B}=\mathcal{B}_{+} \oplus \mathcal{B}_{-}$. Dually, the special fiber of the Hitchin fibration for $S L_{2}$ has two components, so that an $A$-brane supported on this fiber can split up as a sum of two $A$-branes, each supported on one component. Since the components are simply connected, an $A$-brane of rank 1 supported on one of them has no moduli. This is dual to the fact that the fractional branes $\mathcal{B}_{+}$and $\mathcal{B}_{-}$have no moduli. But the brane $\mathcal{B}=\mathcal{B}_{+} \oplus \mathcal{B}_{-}$has moduli (since it can be deformed away from the singularity), and dually, the sum $\mathcal{A}$ of the two $A$-branes can similarly be deformed.

If we deform $\mathcal{B}$ to a skyscraper sheaf supported at a smooth point $r^{*}$ of a nearby Hitchin fiber, then $\mathcal{A}$ deforms to a rank $1 A$-brane supported on the dual Hitchin fiber, with a flat unitary line bundle determined by $r^{*}$. It is also instructive to see what happens if we deform $\mathcal{B}$ to a smooth point $r^{*}$ of the same singular fiber. Then the dual $A$-brane is a flat unitary line bundle on the same dual (singular) Hitchin fiber. However, this flat line bundle now has nontrivial monodromies linking the two irreducible components of the fiber, and therefore they can no longer be "pulled apart." In other words, a generic rank $1 A$-brane on the singular Hitchin fiber is actually irreducible, in agreement with the fact that it corresponds to a rank $1 B$-brane supported at a smooth point, which is also irreducible.

3.6.2. Relation between the two components As hyper-Kahler manifolds, the two components $\mathcal{M}_{\mathrm{H}}\left(\mathrm{SO}_{3}, 0\right)$ and $\mathcal{M}_{\mathrm{H}}\left(S O_{3}, \theta\right)$ are distinct. But if 
we view them purely as complex symplectic manifolds in complex structure $I$, then they are actually isomorphic as long as $\sigma \neq 0$.

One can map between them by making a $\varphi$-invariant Hecke modification at the point $p$. This concept is developed more fully in Section 5.3.3. In brief, decompose $E$ near $p$ as $\mathcal{L}_{1} \oplus \mathcal{L}_{2}$, where $\mathcal{L}_{1}$ and $\mathcal{L}_{2}$ are line bundles that are $\varphi$-invariant, in the sense that $\varphi: E \rightarrow E \otimes K \operatorname{maps} \mathcal{L}_{i} \rightarrow \mathcal{L}_{i} \otimes K$, for $i=1,2$. Order the $\mathcal{L}_{i}$ so that the fiber of $\mathcal{L}_{1}$ at $p$ is the eigenspace of $\sigma$ with eigenvalue $+\sigma_{0}$. (This is the step that requires $\sigma \neq 0$.) Consider the operation that leaves $(E, \varphi)$ unchanged away from $p$ and acts near $p$ as $\mathcal{L}_{1} \oplus \mathcal{L}_{2} \rightarrow \mathcal{L}_{1}(p) \oplus \mathcal{L}_{2}$. When viewed as a transformation of the $S O_{3}$ Higgs bundle $(W, \varphi)$ (with $W=\operatorname{ad}(E)$ ), this operation establishes the isomorphism between the two components of $\mathcal{M}_{\mathrm{H}}\left(\mathrm{SO}_{3}\right)$. This isomorphism commutes with the Hitchin fibration, since the Hecke modification does not change the characteristic polynomial of $\varphi$.

Going back to Hitchin's equations for ramified Higgs bundles, with the singularity postulated in Equation (3.4), the $\varphi$-invariant Hecke modification is equivalent to a shift of $\alpha$ by a lattice vector. (One can compensate for such a shift by a gauge transformation that is discontinuous at $r=0$ and has the effect of changing the natural extension of the bundle over that point. See [30, Section 2.1].) In general [63], $\mathcal{M}_{\mathrm{H}}$ when viewed as a complex symplectic manifold in complex structure $I$ is independent of $\alpha$ as long as $\sigma$ is regular. The equivalence between the two components of $\mathcal{M}_{\mathrm{H}}\left(\mathrm{SO}_{3}\right)$ is a special case of this. For $\sigma$ nonregular (which for $\mathrm{SO}_{3}$ means $\sigma=0$ ), the two components of $\mathcal{M}_{\mathrm{H}}\left(\mathrm{SO}_{3}\right)$ are birational but not isomorphic.

For unramified $\mathrm{SO}_{3}$ Higgs bundles, there is no such relation between the two components.

\section{7. $\mathrm{O}_{2}$-bundles}

Our next task is to interpret better the singularities that we have found in the $\mathrm{SO}_{3}$ moduli space.

In general, for generic ${ }^{17} \sigma$, singularities of the moduli space $\mathcal{M}_{\mathrm{H}}$ of stable ramified Higgs bundles come entirely from automorphisms. If a Higgs bundle $(E, \varphi)$ has a nontrivial finite automorphism group $\Gamma$, we should expect the corresponding point in $\mathcal{M}_{\mathrm{H}}$ to be a singular point. If $\Gamma$ is a finite group, the singularity will be an orbifold singularity, locally of the form $\mathbb{C}^{2 n} / \Gamma$ with some $n$ and some linear action of $\Gamma$ on $\mathbb{C}^{2 n}$. If $\Gamma$ has positive dimension,

\footnotetext{
${ }^{17}$ For nonregular $\sigma, \mathcal{M}_{\mathrm{H}}$ also has local singularities described in [30, Section 3.6].
} 
the singularity is typically (but not always) more severe than an orbifold singularity.

In the present case, we have encountered some $A_{1}$ orbifold singularities, and this strongly suggests that the corresponding $\mathrm{SO}_{3}$ Higgs bundles $(W, \varphi)$ have automorphism group $\Gamma=\mathbb{Z}_{2}$. To describe Higgs bundles with this automorphism group, we will use the correspondence between Higgs bundles and local systems given by Hitchin's equations. This correspondence preserves the automorphism group, so a Higgs bundle with automorphism group $\mathbb{Z}_{2}$ corresponds to a local system with automorphism group $\mathbb{Z}_{2}$.

The reason that it is possible to have an $\mathrm{SO}_{3}$ local system with automorphism group $\mathbb{Z}_{2}$ is that $\mathrm{SO}_{3}$ contains the subgroup $\mathrm{O}_{2}$, consisting of $\mathrm{SO}_{3}$ elements of the form

$$
\left(\begin{array}{rrr}
* & * & 0 \\
* & * & 0 \\
0 & 0 & \pm 1
\end{array}\right),
$$

where the upper left block is an element of $\mathrm{O}_{2}$, and the sign in the lower right corner is chosen so that the determinant equals +1 . $\mathrm{O}_{2}$ has two components topologically; the component that is connected to the identity consists of group elements of lower right matrix entry +1 , and the disconnected component consists of elements for which that entry is -1 . The subgroup of $\mathrm{SO}_{3}$ that commutes with $\mathrm{O}_{2}$ is $\mathbb{Z}_{2}$, generated by

$$
\left(\begin{array}{rrr}
-1 & 0 & 0 \\
0 & -1 & 0 \\
0 & 0 & 1
\end{array}\right)
$$

So an $\mathrm{SO}_{3}$ local system whose structure group reduces to $\mathrm{O}_{2}$ but which is otherwise generic will have automorphism group $\mathbb{Z}_{2}$.

Let us describe local systems on a Riemann surface $C$ of genus 1 , first in the unramified case. Such a local system is determined up to isomorphism by the monodromy elements $V_{1}$ and $V_{2}$ around two one-cycles in $C$. As the fundamental group of $C$ is abelian, they obey

$$
V_{1} V_{2} V_{1}^{-1} V_{2}^{-1}=1
$$

Now suppose that there is a single ramification point $p$, with a specified conjugacy class $M$ for the monodromy around $p$. Then (3.23) is modified to

$$
V_{1} V_{2} V_{1}^{-1} V_{2}^{-1}=M
$$


In our case, the monodromy is $M=\exp (-2 \pi(\alpha-i \gamma))$, as in in Equation (3.5). If $V_{1}$ and $V_{2}$ take values in $O_{2}$ and obey (3.24), then $M$ must take values in the connected component of $\mathrm{O}_{2}$ and has the form

$$
M=\left(\begin{array}{lll}
* & * & 0 \\
* & * & 0 \\
0 & 0 & 1
\end{array}\right) .
$$

For such an $M$, we want to find $V_{1}$ and $V_{2}$, taking values in $O_{2}$, and obeying (3.24).

An $\mathrm{SO}_{3}$ local system $W$ whose structure group actually reduces to $\mathrm{O}_{2}$ splits up as $U \oplus \mathcal{S}$, where $W$ is a rank 2 local system (with structure group $\left.\mathrm{O}_{2} \subset G L_{2}\right)$ and $\mathcal{S} \cong \operatorname{det} U$ is a line bundle of order 2 . If $\mathcal{S}$ is trivial, then $V_{1}$ and $V_{2}$ both take values in the connected component of $O_{2}$. Since this connected component is the abelian group $\mathrm{SO}_{2}$, Equation (3.24) is then impossible to obey for $M \neq 1$.

So we must take $\mathcal{S}$ to be a nontrivial line bundle of order 2. This means that either $V_{1}$ or $V_{2}$, or both, takes values in the disconnected component of $\mathrm{O}_{2}$. We can choose the two one-cycles with holonomies $V_{1}$ and $V_{2}$ so that $V_{1}$ takes values in the disconnected component and $V_{2}$ in the connected component. Any element of the disconnected component is conjugate to

$$
V_{1}=\left(\begin{array}{rrr}
1 & 0 & 0 \\
0 & -1 & 0 \\
0 & 0 & -1
\end{array}\right)
$$

With this choice of $V_{1}$, we have $V_{1} V_{2} V_{1}^{-1}=V_{2}^{-1}$, so (3.24) reduces to

$$
V_{2}^{2}=M^{-1}
$$

where $\mathrm{M}$ and $\mathrm{V}_{2}$ take values in $\mathrm{SO}_{2}$.

For each $M$, this last equation has precisely two solutions, differing (in $S_{2}$ ) by $V_{2} \rightarrow-V_{2}$. Hence, for each nontrivial line bundle $\mathcal{S}$ of order 2 , we can construct precisely two $\mathrm{SO}_{3}$ local systems each with a group of automorphisms $R \cong \mathbb{Z}_{2}$.

These two local systems, however, differ by the value of $w_{2}$. Hence, one of them corresponds to a $\mathbb{Z}_{2}$ orbifold singularity on the appropriate fiber of $\mathcal{M}_{\mathrm{H}}\left(C ; S O_{3}, 0\right)$, and one corresponds to such a singularity on the corresponding fiber of $\mathcal{M}_{\mathrm{H}}\left(C ; S O_{3}, \theta\right)$. 
To explain why the two choices of $V$ correspond to topologically inequivalent bundles, consider the special case $M=1$. In this case, the two possibilities for $V_{2}$ are

$$
V_{2}^{(1)}=1, V_{2}^{(2)}=\left(\begin{array}{rrr}
-1 & 0 & 0 \\
0 & -1 & 0 \\
0 & 0 & 1
\end{array}\right)
$$

In either case, $V_{1}$ and $V_{2}$ are diagonal, so $W$ splits as a direct sum of three rank 1 local systems $\mathcal{S}_{1}, \mathcal{S}_{2}, \mathcal{S}_{3}$. We therefore have

$$
w_{2}(W)=\sum_{1 \leq i<j \leq 3} w_{1}\left(\mathcal{S}_{i}\right) \cup w_{1}\left(\mathcal{S}_{j}\right)
$$

If $V_{2}=1$, then $\mathcal{S}_{1}$ is trivial and $\mathcal{S}_{2}$ and $\mathcal{S}_{3}$ are isomorphic. Hence $w_{2}(W)=w_{1}\left(\mathcal{S}_{2}\right)^{2}$, and this is zero since $x^{2}=0$ for any $x \in H^{1}\left(C, \mathbb{Z}_{2}\right)$.

If $V_{2}=V_{2}^{(2)}$, then $\mathcal{S}_{1}, \mathcal{S}_{2}$ and $\mathcal{S}_{3}$ are the three distinct nontrivial line bundles of order 2 over $C$. If $x=w_{1}\left(\mathcal{S}_{1}\right), y=w_{1}\left(\mathcal{S}_{2}\right)$, then we have $w_{1}\left(\mathcal{S}_{3}\right)=$ $x+y$, since $S_{3} \cong S_{1} \otimes S_{2} . \quad x$ and $y$ are a basis for $H^{1}\left(C, \mathbb{Z}_{2}\right) \cong \mathbb{Z}_{2} \oplus \mathbb{Z}_{2}$, and $\theta=x \cup y$ generates $H^{2}\left(C, \mathbb{Z}_{2}\right) \cong \mathbb{Z}_{2}$. Evaluation of (3.29) now gives $w_{2}(W)=x \cup y$, so in particular $w_{2}(W) \neq 0$.

Thus, for any choice of nontrivial line bundle $\mathcal{S}$ of order 2 , and any choice of $w_{2}(W)$, we find one $S_{3}$ local system with automorphism group $\mathbb{Z}_{2}$. This accounts for the singularities that we found in Section 3.6 for $w_{2}=0$, as well as the singularities that we will find for $w_{2} \neq 0$.

Remark 3.2. In the example with $w_{2} \neq 0$, instead of thinking of $W=\mathcal{S}_{1} \oplus$ $\mathcal{S}_{2} \oplus \mathcal{S}_{3}$ as an $S_{3}$ local system, we can understand it as a flat bundle with structure group the compact form of $\mathrm{SO}_{3}$, or equivalently, as a stable holomorphic $\mathrm{SO}_{3}$ bundle. As such, $W$ is the unique topologically nontrivial flat bundle or stable holomorphic $\mathrm{SO}_{3}$ bundle on a curve of genus 1 . In particular, it has no deformations; for instance, $H^{1}(C, W)=\oplus_{i=1}^{3} H^{1}\left(C, \mathcal{S}_{i}\right)=0$, as the cohomology of a nontrivial line bundle vanishes for $C$ of genus 1 .

Remark 3.3. We have been too cavalier in describing the distinction between the two components of $\mathcal{M}_{\mathrm{H}}\left(C ; S O_{3}\right)$. In the unramified case, $w_{2}(W)$ is a well-defined topological invariant. But in the ramified case, the definition of $w_{2}(W)$ depends on a choice of extension of $W$ (at least as a topological bundle) over the ramification point $p$. The moduli space $\mathcal{M}_{\mathrm{H}}\left(\mathrm{C} ; \mathrm{SO}_{3}\right)$ has two components, each of which has the singularities that we have described, but it is oversimplified to claim that one corresponds 
to $w_{2}(W)=0$ and one to $w_{2}(W) \neq 0$. Indeed, the two components are exchanged under monodromy of $\mathrm{M}$ in the group $\mathrm{SO}_{2}$, whose fundamental group is $\mathbb{Z}$. The monodromy in $M$ can be achieved by varying the parameter $\alpha$, and thus is equivalent to the operation described in Section 3.6.2.

3.7.1. Relation to geometric endoscopy What we have found is, again, fully in keeping with expectations from Section 2.2. An $\mathrm{SO}_{3}$ local system whose structure group reduces to $O_{2}$ has automorphism group $\mathbb{Z}_{2}$. It corresponds to an $A_{1}$ singularity of $\mathcal{M}_{\mathrm{H}}\left(C ; S O_{3}\right)$. So the category of $B$-branes associated with this local system is generated by two objects $\mathcal{B}_{+}$ and $\mathcal{B}_{-}$. In contrast, $\mathcal{M}_{\mathrm{H}}\left(C ; S L_{2}\right)$ is smooth; some exceptional fibers of the Hitchin fibration are singular, but the singularities of the fibers are smooth points of the total space.

\subsection{Second component}

In Section 3.7, we have constructed $\mathrm{SO}_{3}$ local systems with $w_{2} \neq 0$ whose structure group reduces to $\mathrm{O}_{2}$. These should correspond to $A_{1}$ singularities of $\mathcal{M}_{\mathrm{H}}\left(C ; \mathrm{SO}_{3}, \theta\right)$.

It will be useful to give a direct description of this space. $\mathcal{M}_{\mathrm{H}}\left(C ; S_{3}, \theta\right)$ parametrizes ramified Higgs bundles $(W, \varphi)$, where $W$ is an $S_{3}$-bundle with nonzero $w_{2}$. It will be sufficient for our purposes to construct a Zariski open set in this moduli space corresponding to Higgs bundles for which $W$ is stable. According to Remark 3.2, this means that $W=\mathcal{S}_{1} \oplus \mathcal{S}_{2} \oplus \mathcal{S}_{3}$ is the direct sum of the three nontrivial line bundles of order 2 . We can take $\mathcal{S}_{i}=\mathcal{O}(p) \otimes \mathcal{O}\left(q_{i}\right)^{-1}$, where $q_{i}$ is the point of order 2 given by $(x, y)=\left(e_{i}, 0\right)$. As an $\mathrm{SO}_{3}$ bundle, $W$ should have a nondegenerate quadratic form, which we will denote as a trace (thinking of $W$ as an adjoint bundle). This can be defined as follows: if $s=s_{1} \oplus s_{2} \oplus s_{3}$ is a section of $W=\mathcal{S}_{1} \oplus \mathcal{S}_{2} \oplus \mathcal{S}_{3}$, then the quadratic form is $\operatorname{Tr} s^{2}=\sum_{i} s_{i}^{2} /\left(x-e_{i}\right)$. The idea is that $s_{i}^{2}$ is a section of $\mathcal{O}(p)^{2} \otimes \mathcal{O}\left(q_{i}\right)^{-2}$, which can be trivialized by dividing by $x-e_{i}$.

The bundle $W$ has an automorphism group $Q=\mathbb{Z}_{2} \times \mathbb{Z}_{2}$. Indeed, as a flat bundle, $W$ has monodromies $V_{1}$ and $V_{2}^{(2)}$ presented in Equations (3.26) and (3.28) and $Q$ is the subgroup of $\mathrm{SO}_{3}$ consisting of diagonal matrices with diagonal entries \pm 1 .

To construct $\mathcal{M}_{\mathrm{H}}\left(C ; S_{3}, \theta\right)$ (or rather a Zariski open set corresponding to stable bundles $E$ ), we must consider all Higgs fields $\varphi \in H^{0}(C, K \otimes$ $\mathcal{O}(p) \otimes W)$, impose a suitable condition on the polar part of $\operatorname{Tr} \varphi^{2}$ and divide by $Q$. Before trying to do this, let us discuss what will happen if we do not divide by $Q$. 
From the point of view of complex algebraic geometry, instead of considering $S L_{2}$ Higgs bundles $(E, \varphi)$ where $E$ is a rank 2 holomorphic bundle with det $E$ trivial, it is natural to pick a fixed line bundle $\mathcal{L}$ and consider Higgs bundles $(E, \varphi)$ with $\operatorname{det} E=\mathcal{L}$. If one replaces $\mathcal{L}$ by $\mathcal{L} \otimes \mathcal{N}^{2}$, one can compensate for this by $E \rightarrow E \otimes \mathcal{N}$. Modulo this operation, all that really matters about $\mathcal{L}$ is its degree modulo 2. Apart from the familiar case of $\mathcal{L}=\mathcal{O}$, we can consider a second component with (say) $\operatorname{det} E=\mathcal{O}(p)$. We write $\mathcal{M}_{\mathrm{H}}\left(C ; S L_{2}^{*}\right)$ for the moduli space of stable Higgs bundles of this type. We sometimes refer to this as the improper component of the $S L_{2}$ moduli space (and we refer to the moduli space of ordinary $S L_{2}$ Higgs bundles $(E, \varphi)$ with $\operatorname{det} E$ trivial as the proper component). If $(E, \varphi)$ is a Higgs bundle with $\operatorname{det} E=\mathcal{O}(p)$, then, upon setting $W=\operatorname{ad}(E)$, we get an $S_{3}$ Higgs bundle $(W, \varphi)$ with $w_{2}(W) \neq 0$. All $(W, \varphi)$ can arise this way, and $(E, \varphi)$ is determined uniquely from $(W, \varphi)$ up to twisting $E$ by a line bundle of order 2 . So $\mathcal{M}_{\mathrm{H}}\left(C ; S L_{2}^{*}\right)$ can be related to $S_{3}$ Higgs bundles by analogy with (3.20):

$$
\mathcal{M}_{\mathrm{H}}\left(C ; S O_{3}, \theta\right)=\frac{\mathcal{M}_{\mathrm{H}}\left(C ; S L_{2}^{*}\right)}{Q} .
$$

From the point of view of differential geometry, $\mathcal{M}_{\mathrm{H}}\left(C ; S L_{2}^{*}\right)$ can be constructed by solving Hitchin's equations with gauge group the compact group $\mathrm{SO}_{3}$ on a bundle with $w_{2} \neq 0$ and dividing only by those gauge transformations that can be lifted to $S U(2)$.

Remark 3.4. The reasoning of Remark 3.3 may also be applied to the group $S L_{2}$ to show that $\mathcal{M}_{\mathrm{H}}\left(C ; S L_{2}\right)$ and $\mathcal{M}_{\mathrm{H}}\left(C ; S L_{2}^{*}\right)$ are isomorphic as complex symplectic manifolds in complex structure $I$.

Now let us give an explicit description. We consider a Higgs field $\varphi$ that is a section of $K \otimes \mathcal{O}(p) \otimes W=\oplus_{i=1}^{3} K \otimes \mathcal{O}(p)^{2} \otimes \mathcal{O}\left(q_{i}\right)^{-1}$. For each $i$, we can pick a section $u_{i}$ of $K \otimes \mathcal{O}(p)^{2} \otimes \mathcal{O}\left(q_{i}\right)^{-1}$, namely $u_{i}=(d x / y)\left(x-e_{i}\right)$, with $\operatorname{Tr} u_{i}^{2}=(d x / y)^{2}\left(x-e_{i}\right)$. The general form of the Higgs field is

$$
\varphi=\sum_{i=1}^{3} a_{i} u_{i}
$$

with complex constants $a_{i}$. This gives

$$
\operatorname{Tr} \varphi^{2}=\left(\frac{d x}{y}\right)^{2} \sum_{i=1}^{3} a_{i}^{2}\left(x-e_{i}\right)
$$


As in Equation (3.11), what multiplies $(d x / y)^{2}$ is a first-order polynomial in $x$. Letting $z \sim x^{-1 / 2}$ be a local parameter at infinity, the polar part of $\operatorname{Tr} \varphi^{2}$ is $4(d z / z)^{2} \sum_{i} a_{i}^{2}$. Setting this to $2 \sigma_{0}^{2}(d z / z)^{2}$, we require

$$
\sum_{i} a_{i}^{2}=\frac{\sigma_{0}^{2}}{2} .
$$

This affine quadric describes a Zariski open set in $\mathcal{M}_{\mathrm{H}}\left(C ; S L_{2}^{*}\right)$ (in Section 3.8.1, we divide by $Q=\mathbb{Z}_{2} \times \mathbb{Z}_{2}$ and give a similar description of $\left.\mathcal{M}_{\mathrm{H}}\left(C ; S O_{3}, \theta\right)\right)$.

The constant term multiplying $(d x / y)^{2}$ on the right-hand side of (3.32) is $-\sum_{i} e_{i} a_{i}^{2}$. This enables us to describe the Hitchin fibration; it is the map from $\left(a_{1}, a_{2}, a_{3}\right)$ to

$$
w_{0}=-\sum_{i} e_{i} a_{i}^{2}
$$

A fiber of the Hitchin fibration is given by the intersection of the two quadrics (3.33) and (3.34). For the same reasons as in our discussion of $S L_{2}$, the parameter $\sigma_{0}$ can be scaled out of these equations, assuming that it is nonzero. We set $w_{0}=-\sigma_{0}^{2} w / 2$, and $b_{i}=a_{i}\left(\sqrt{2} / \sigma_{0}\right)$ to put the two quadrics in the form

$$
\begin{aligned}
b_{1}^{2}+b_{2}^{2}+b_{3}^{2} & =1 \\
e_{1} b_{1}^{2}+e_{2} b_{2}^{2}+e_{3} b_{3}^{2} & =w .
\end{aligned}
$$

For generic $w$, this intersection is a smooth curve of genus 1 , with some points omitted because we have assumed $W$ to be stable. For what values of $w$ is the fiber singular? If $f=\sum_{i} b_{i}^{2}-1, g=\sum_{i} e_{i} b_{i}^{2}-w$, then a singularity of the fiber is a point with $f=g=d f \wedge d g=0$. A short calculation shows that $d f \wedge d g=0$ precisely if two of $b_{1}, b_{2}$ and $b_{3}$ vanish. If $b_{i}$ is nonvanishing for some $i$ and $b_{j}=0$ for $j \neq i$, then we must have

$$
w=e_{i}
$$

and

$$
b_{i}= \pm 1
$$

Thus, there are precisely three singular fibers, one for each choice of $i$, just as in Section 3.4. The singular fibers are at $w=e_{i}, i=1,2,3$, just as we found 
for the other component of the moduli space. These facts of course agree with the relation between the two components claimed in Section 3.6.2.

Moreover, each singular fiber $\boldsymbol{F}_{w}$ contains two singular points, given in Equation (3.37). The singular fibers consist of two components of genus 0 joined at two double points. To see this, take $i=1$. If $w=e_{1}$, then a linear combination of the equations $f=0$ and $g=0$ gives $\left(e_{2}-e_{1}\right) b_{2}^{2}+$ $\left(e_{3}-e_{1}\right) b_{3}^{2}=0$ or

$$
b_{2}= \pm b_{3} \sqrt{-\frac{e_{3}-e_{1}}{e_{2}-e_{1}}}
$$

This describes a curve $\boldsymbol{F}_{w, 0}$ that is a union of two genus 0 components meeting at one point, $b_{2}=b_{3}=0$. Now solving for $b_{1}$ via $b_{1}^{2}=1-b_{3}^{2}\left(e_{2}-\right.$ $\left.e_{3}\right) /\left(e_{2}-e_{1}\right)$ gives a double cover of $\boldsymbol{F}_{w, 0}$. The double cover, which is the fiber $\boldsymbol{F}_{w}$ of the Hitchin fibration, is branched over two points in each component of $\boldsymbol{F}_{w, 0}$. A double cover of a curve of genus 0 branched at two points is still of genus 0 . So $\boldsymbol{F}_{w}$ consists of two components of genus 0 , meeting at the two points $b_{2}=b_{3}=0, b_{1}= \pm 1$.

3.8.1. $\mathrm{SO}_{3}$ moduli space What we have constructed so far is a Zariski open set in $\mathcal{M}_{\mathrm{H}}\left(C ; S L_{2}^{*}\right)$, together with its Hitchin fibration. We can also divide by $Q$, which acts by pairwise sign changes of the $b_{i}$, and get a Zariski open set in $\mathcal{M}_{\mathrm{H}}\left(C ; S O_{3}, \theta\right)$.

The invariants under pairwise sign changes of the $b_{i}$ are $b_{1}^{2}, b_{2}^{2}, b_{3}^{2}$, and $z=b_{1} b_{2} b_{3}$. They obey $z^{2}=b_{1}^{2} b_{2}^{2} b_{3}^{2}$. Using the two equations (3.35), one can solve for $b_{i}^{2}, i=1,2,3$ as linear functions of $t=\sum_{i=1}^{3} e_{i}^{2} b_{i}^{2}$. The equation $z^{2}=b_{1}^{2} b_{2}^{2} b_{3}^{2}$ becomes

$$
z^{2}=-\prod_{1 \leq i<j \leq 3} \frac{\left(t-w\left(e_{i}+e_{j}\right)+e_{i} e_{j}\right)}{\left(e_{i}-e_{j}\right)^{2}} .
$$

This describes the $\mathrm{SO}_{3}$ moduli space (except that one must include points with $t, z=\infty$ for fixed $w)$.

Let us use this description to find the singular fibers of the Hitchin fibration for $\mathcal{M}_{\mathrm{H}}\left(C ; S O_{3}, \theta\right)$. The cubic polynomial on the right-hand side of (3.39) has distinct roots unless $w=e_{i}$ for some $i$, in which case precisely two of the roots coincide. So the only singular fibers are for $w=e_{i}$, and they are nodal cubic curves, that is, copies of $\mathbb{C P}^{1}$ with two points identified. By expanding the cubic equation (3.39) near a double point, it is not hard to verify that the double points of the fiber are actually $A_{1}$ singularities of $\mathcal{M}_{\mathrm{H}}\left(C ; S O_{3}, \theta\right)$. This is the same result that we found at the end of Section 
3.6 for the singular fibers of $\mathcal{M}_{\mathrm{H}}\left(C ; \mathrm{SO}_{3}, 0\right)$. This is, of course, completely in keeping with the claim in Section 3.6.2 that the two components of the moduli space are equivalent in complex structure $I$.

\subsection{Relation to the cotangent bundle}

One of the most important properties [33] of the moduli space $\mathcal{M}_{\mathrm{H}}$ of stable Higgs bundles (without ramification) is that it can be approximated as $T^{*} \mathcal{M}$, where $\mathcal{M}$ is the moduli space of stable bundles. $(\mathcal{M}$ is a Zariski open set the moduli space that parametrizes stable and semi-stable bundles.) The reason for this is that the cotangent space to $\mathcal{M}$, at a point corresponding to a stable bundle $E$, is $H^{0}(C, K \otimes \operatorname{ad}(E))$. So a point in $T^{*} \mathcal{M}$ is a pair $(E, \varphi)$, or in other words a Higgs bundle. This gives an embedding of $T^{*} \mathcal{M}$ as a Zariski open set in $\mathcal{M}_{\mathrm{H}}$. This map is not surjective because a Higgs bundle $(E, \varphi)$ may be stable or semi-stable, and so represent a point in $\mathcal{M}_{\mathrm{H}}$, even if the underlying bundle $E$ is not stable.

This has an analog for ramified Higgs bundles. In this case, one takes $\mathcal{M}$ to be the moduli space of stable bundles with parabolic structure at a point $p$, and $\mathcal{M}_{\mathrm{H}}$ to be the moduli space of stable ramified Higgs bundles. (For a generic choice of the parabolic weight - the parameter called $\alpha$ in (3.4) every semi-stable parabolic bundle is stable.) Then $\mathcal{M}_{\mathrm{H}}$ has a Zariski open set that is not quite $T^{*} \mathcal{M}$ but is an affine symplectic deformation of $T^{*} \mathcal{M}$, as described in [30, section 3.6]. We denote such an affine symplectic deformation as $\widetilde{T}^{*} \mathcal{M}$. Here $\widetilde{T}^{*} \mathcal{M}$ denotes a complex symplectic variety with a map to $\mathcal{M}$, such that locally in $\mathcal{M}, \widetilde{T}^{*} \mathcal{M}$ is symplectically isomorphic to $T^{*} \mathcal{M}$. For a detailed description of an example, see Section 3.9.1.

For applications to geometric Langlands, it is important to restrict the fibers of the Hitchin fibration from $\mathcal{M}_{\mathrm{H}}$ to $T^{*} \mathcal{M}$ or $\widetilde{T}^{*} \mathcal{M}$, since this is an essential step in interpreting $A$-branes in terms of $\mathcal{D}$-modules, as discussed in Section 2.3). So let us carry out this step for our example of $S L_{2}$ Higgs bundles on a curve $C$ of genus 1 with one point $p$ of ramification. Of course, one can divide by $Q$ and make a similar discussion for $\mathrm{SO}_{3}$.

3.9.1. Calculation These questions depend only on $\mathcal{M}_{\mathrm{H}}$ as a complex symplectic manifold in complex structure $I$. As such, the two components are equivalent, according to Section 3.6.2, so from now on, we will consider the improper component.

We have explicitly described the stable Higgs bundles $(E, \varphi)$, where $\operatorname{det} E=\mathcal{O}(p)$, and $E$ is stable. $E$ is uniquely determined up to isomorphism and we have found the space of Higgs bundles of this kind to be 
precisely the affine quadric

$$
b_{1}^{2}+b_{2}^{2}+b_{3}^{2}=1
$$

which we will denote for the moment as $\mathcal{M}_{\mathrm{H}}^{0}$. It differs slightly from $\mathcal{M}_{\mathrm{H}}$, which also parametrizes stable pairs $(E, \varphi)$, where the underlying bundle $E$ is unstable.

In this situation, what is the moduli space $\mathcal{M}$ of stable parabolic bundles? The bundle $E$ has no deformations, but its parabolic structure at the point $p$ can vary. The choice of parabolic structure is the choice of a complex line $\Psi$ in the two-dimensional vector space $E_{p}$, the fiber of $E$ at $p$. So $\mathcal{M}$ is a copy of $\mathbb{C P}^{1}$, parametrizing the choice of $\Psi$.

With $E$ as above, suppose that we are given a ramified Higgs bundle $(E, \varphi)$, where $\varphi$ has a pole at $p$ whose residue has eigenvalues $\pm \sigma_{0}$. This determines a parabolic structure on $E$, by setting $\Psi$ to be the eigenspace of the residue with eigenvalue $+\sigma_{0}$. So there is a natural map from $\mathcal{M}_{\mathrm{H}}^{0}$ to $\mathcal{M}$, taking $(E, \varphi)$ to the parabolic bundle with this $\Psi$. This map has no holomorphic section, since given the bundle $E$ and the choice of $\Psi$, there is no natural way to produce a $\varphi$ with the right pole. It is this that leads to the affine deformation.

Alternatively, we could get a second and equally natural parabolic structure and map $\mathcal{M}_{\mathrm{H}}^{0} \rightarrow \mathcal{M}$ by using the eigenspace with eigenvalue $-\sigma_{0}$. This would ultimately lead to a different functor from $A$-branes to twisted $\mathcal{D}$-modules. In general, in the ramified case of geometric Langlands, the mirror symmetry between the $B$-model and the $A$-model is uniquely determined, but the mapping from $A$-branes to twisted $\mathcal{D}$-modules depends on a choice of Borel subgroup containing the local monodromy. The different choices lead to $\mathcal{D}$-modules with different twistings, related by a Weyl transformation.

In the present context, the choice of Borel subgroup amounts to the choice of eigenvalue $+\sigma_{0}$ or $-\sigma_{0}$. (The isomorphism between the two components of $\mathcal{M}_{\mathrm{H}}$ also depends on this choice of sign.) For $S L_{n}$, the analog would be to pick an ordering of the eigenvalues of the polar part of $\varphi$.

The map from the affine quadric $b_{1}^{2}+b_{2}^{2}+b_{3}^{2}=1$ to $\mathbb{C P}^{1}$ can of course be described without talking about Higgs bundles. The equation $b_{1}^{2}+b_{2}^{2}+b_{3}^{2}=1$ is equivalent to the statement that the matrix

$$
M=\left(\begin{array}{cc}
b_{1} & b_{2}-i b_{3} \\
b_{2}+i b_{3} & -b_{1}
\end{array}\right)
$$


has eigenvalues \pm 1 . Taking $\Psi$ to be the space of solutions of the equation $M \psi=\psi$, we get a map from the affine quadric to $\mathbb{C P}^{1}$. If we set $\psi=\left({ }_{z}^{1}\right)$, we find that in affine coordinates, the map to $\mathbb{C P}^{1}$ can be described by $z=\left(1-b_{1}\right) /\left(b_{2}-i b_{3}\right)=\left(b_{2}+i b_{3}\right) /\left(1+b_{1}\right)$.

The fibers of the map from $\mathcal{M}_{\mathrm{H}}^{0}$ to $\mathbb{C P}^{1}$ are copies of $\mathbb{C}$. For example, the fiber for $z=0$ is given by $b_{1}=1, b_{2}+i b_{3}=0$, and is parametrized by $b_{2}$. However, $\mathcal{M}_{\mathrm{H}}^{0}$ is not a holomorphic line bundle over $\mathbb{C P}^{1}$, because the map from $\mathcal{M}_{\mathrm{H}}^{0}$ to $\mathbb{C P}^{1}$ has no holomorphic section. Rather, the structure group of the bundle $\mathcal{M}_{\mathrm{H}}^{0} \rightarrow \mathbb{C P}^{1}$ is the group of affine linear motions of $\mathbb{C}$, that is the group of transformations of the form $x \rightarrow a x+b, a \in \mathbb{C}^{\times}, b \in \mathbb{C}$. We might describe $\mathcal{M}_{\mathrm{H}}^{0}$ as an affine bundle of rank 1 .

The group of affine linear motions has a homomorphism to $\mathbb{C}^{\times}$, by forgetting $b$. So to an affine bundle of rank 1 , there is always a canonically associated fiber bundle whose structure group is $\mathbb{C}^{\times}$. In the case of the quadric, the associated variety is the cotangent bundle of $\mathbb{C P}^{1}$. The quadric itself admits a holomorphic symplectic form: ${ }^{18}$

$$
\Omega=i \sigma_{0} \frac{d b_{1} \wedge d b_{2}}{b_{3}}
$$

which is part of the hyper-Kahler structure on the moduli space. Locally on $\mathbb{C P}^{1}$, one can pick a section of the fibration $\mathcal{M}_{\mathrm{H}}^{0} \rightarrow \mathbb{C P}^{1}$ and identify $\mathcal{M}_{\mathrm{H}}^{0}$ with the cotangent bundle of $\mathbb{C P}^{1}$.

To do so explicitly on the Zariski open set with $b_{1} \neq-1$, where

$$
z=\frac{1-b_{1}}{b_{2}-i b_{3}}=\frac{b_{2}+i b_{3}}{1+b_{1}}
$$

is defined, let $v=-\sigma_{0}\left(b_{2}-i b_{3}\right)$. A small computation shows that $\Omega=d z \wedge d v$, so away from $b_{1}=-1$, we can embed $\mathbb{C P}^{1}$ in $\mathcal{M}_{\mathrm{H}}^{0}$ as the locus $v=0$ and identify $\mathcal{M}_{\mathrm{H}}^{0}$ with the cotangent bundle of the $z$-plane. This identification does not extend ${ }^{19}$ over $z=\infty$, and globally $\mathcal{M}_{\mathrm{H}}^{0}$ is what we call an affine deformation of the cotangent bundle. It is convenient to also introduce $\widetilde{v}=v / \sigma_{0}=-\left(b_{2}-i b_{3}\right)$.

Since $\mathcal{M}_{\mathrm{H}}^{0}$ is not the cotangent bundle to $\mathcal{M}$, but is an affine deformation thereof, we will denote it henceforth as $\widetilde{T}^{*} \mathcal{M}$.

\footnotetext{
${ }^{18}$ The multiplicative constant $\sigma_{0}$ was determined by integrating $\Omega$ over the twocycle characterized by $b_{1}, b_{2}$ and $b_{3}$ real and comparing to the result of [30, Equation (3.77)], for the cohomology class of $\Omega$.

${ }^{19}$ The fact that $\Omega$ has nonzero periods, as noted in the last footnote, implies that it cannot be globally the symplectic form of a cotangent bundle.
} 
Now let us consider the fibers of the Hitchin fibration. Their intersection with the quadric $\widetilde{T}^{*} \mathcal{M}$ is obtained by supplementing the defining equation of the quadric with the equation

$$
e_{1} b_{1}^{2}+e_{2} b_{2}^{2}+e_{3} b_{3}^{2}=w
$$

giving an algebraic curve $\boldsymbol{F}_{w}$. This curve, of course, can be projected to $\mathcal{M}$ and gives a double cover of $\mathcal{M}$. In general, however, the fibers of the Hitchin fibration are complete (and generically smooth) algebraic varieties. This is an important part of the theory in [33]. We will explain it, in the present context, in Section 5.3 using spectral covers. So the fiber $\boldsymbol{F}_{w}$ of the Hitchin fibration of $\mathcal{M}_{\mathrm{H}}$ is really, for generic $w$, the smooth projective curve that corresponds to the affine curve just described. A few points are missing in the description by affine quadrics.

We can describe $\boldsymbol{F}_{w}$ as a projective curve by simply adding another variable $b_{4}$, where $b_{1}, \ldots, b_{4}$ are understood as homogeneous coordinates on $\mathbb{C P}^{3}$ and obey

$$
\begin{gathered}
\sum_{i=1}^{3} b_{i}^{2}=b_{4}^{2} \\
\sum_{i=1}^{3} e_{i} b_{i}^{2}=w b_{4}^{2} .
\end{gathered}
$$

Missing when one approximates $\mathcal{M}_{\mathrm{H}}$ by an affine deformation of the cotangent bundle are the four points with $b_{4}=0$. These points correspond to stable Higgs bundles $(E, \varphi)$ where the parabolic bundle $E$ is unstable. ${ }^{20}$ They form a single orbit of the group $Q=\mathbb{Z}_{2} \times \mathbb{Z}_{2}$ of pairwise sign changes of $b_{1}, b_{2}, b_{3}$. Explicitly, the values of $z$ corresponding to these four points are

$$
z= \pm \sqrt{\frac{e_{2}-e_{1}}{e_{2}-e_{3}}} \pm \sqrt{\frac{e_{3}-e_{1}}{e_{2}-e_{3}}} .
$$

To gain some insight about the $\mathcal{D}$-modules arising in the geometric Langlands program, we must describe $\boldsymbol{F}_{w}$ as a curve in $\widetilde{T}^{*} \mathcal{M}$. For this, we use the coordinates $z, \widetilde{v}$, and find, after some algebra, that we can describe the

${ }^{20} E$ must have determinant $\mathcal{O}(p)$ and must be of the form $\mathcal{L} \otimes(\mathcal{O}(p) \oplus \mathcal{O})$, where $\mathcal{L}$ is of order 2 , in order for there to exist a stable ramified Higgs bundle $(E, \varphi)$. The four choices of $\mathcal{L}$ lead to the four missing points on each fiber of the Hitchin fibration. 
fiber $\boldsymbol{F}_{w}$ by an explicit quadratic equation for $\widetilde{v}$, of the form

$$
A(z) \widetilde{v}^{2}+B(z) \widetilde{v}+C(z)=0
$$

with

$$
\begin{aligned}
& A(z)=\left(e_{2}-e_{3}\right) z^{4}+\left(4 e_{1}-2 e_{2}-2 e_{3}\right) z^{2}+\left(e_{2}-e_{3}\right) \\
& B(z)=-4 z\left(\left(e_{2}-e_{3}\right) z^{2}+2 e_{1}-e_{2}-e_{3}\right) \\
& C(z)=4\left(\left(e_{2}-e_{3}\right) z^{2}+e_{1}-w\right)
\end{aligned}
$$

Note that

$$
B=-\frac{d A}{d z} .
$$

If we let $z$ approach one of the four values in Equation (3.45), then one of the roots of the quadratic equation for $\widetilde{v}$ goes to infinity. So at any of those values of $z$, a point in the Hitchin fiber is "missing," if we restrict to $\widetilde{T}^{*} \mathcal{M}$. In fact, the four critical values of $z$ are precisely the zeros of the polynomial $A(z)$. At those values of $z, B(z)$ is nonzero, which means that one of the two roots of the quadratic equation (3.46) for $\widetilde{v}$ has a pole, and one does not. It is convenient to express the result in terms of the variable $v=\sigma_{0} \widetilde{v}$ that puts the symplectic form $\Omega$ in a canonical form. Let $z^{*}$ be any one of the zeros of $A(z)$. The behavior of the polar branch of $v$ near $z^{*}$ is $v \sim-\sigma_{0} B(z) / A(z)$, which using Equation (3.48) reduces to

$$
v \sim \sigma_{0} \frac{1}{z-z^{*}}
$$

Remark 3.5. For the geometric endoscopy, we must examine in a similar way the singular fibers of the Hitchin fibration. For example, we take $w=e_{1}$, so that the fiber $\boldsymbol{F}_{e_{1}}$ splits into components $\boldsymbol{F}_{e_{1}}^{ \pm}$defined by the ratio of $b_{2} / b_{3}$, as in Equation (3.38). Compactifying the two components in projective space, we see that of the four points at $b_{4}=0$, two lie on $\boldsymbol{F}_{e_{1}}^{+}$and two on $\boldsymbol{F}_{e_{1}}^{-}$. (This actually follows just from the fact that the four points are an orbit of $Q$, and that $Q$ exchanges the two components.) If we restrict to $\widetilde{T}^{*} \mathcal{M}$, the two curves $\boldsymbol{F}_{e_{1}}^{ \pm}$behave near the two relevant critical values of $z$ precisely as found in the last paragraph. So each fractional $A$-brane has two points with this sort of behavior.

3.9.2. Nilpotent Higgs fields There is a simple way to characterize points in $\mathcal{M}$ over which a fiber $\boldsymbol{F}$ of the Hitchin fibration, when viewed 
as a curve in $T^{*} \mathcal{M}$, goes to infinity. They correspond to stable parabolic bundles $E$ that admit a nonzero nilpotent Higgs field (whose polar residue leaves fixed the parabolic structure of $E$ ). This is relevant for characterizing twisted $\mathcal{D}$-modules arising in geometric Langlands duality [49].

For example, consider the improper component of the $S L_{2}$ moduli space. The Higgs field $\varphi=\sum a_{i} u_{i}$ obeys $\operatorname{Tr} \varphi^{2}=\sum_{i} a_{i}^{2}\left(x-e_{i}\right)$, as we computed in Equation (3.32). So if $0=\sum_{i} a_{i}^{2}=\sum_{i} e_{i} a_{i}^{2}$, which is the condition defining a point in $\boldsymbol{F}$ that does not lie in $T^{*} \mathcal{M}$, then $\operatorname{Tr} \varphi^{2}$ identically vanishes and $\varphi$ is nilpotent.

For the proper component, if $E=\mathcal{L} \oplus \mathcal{L}^{-1}$, then the condition for the Hitchin fiber $\boldsymbol{F}$ to go to infinity in $\widetilde{T}^{*} \mathcal{M}$ is that $\mathcal{L}$ should be of order 2. (This is clear in the original coordinates used in Equation (3.12). $\mathcal{L}$ of order 2 means $x_{0}=\infty$ or $f\left(x_{0}\right)=0$; for such $x_{0}$, with $w_{0}$ fixed, we have $h_{0} \rightarrow \infty$.) On the other hand, if we endow $E$ with a parabolic structure that makes it stable, then a Higgs field whose pole at the point $p$ leaves fixed the parabolic structure cannot be nilpotent unless $\mathcal{L}$ is of order 2 . Indeed, if $\mathcal{L}$ is not of order 2, the general form of this Higgs field is given in Equations (3.8) and (3.9), which show that $\varphi$ can be everywhere nilpotent only if $h_{0}$ vanishes along with either $k_{0}$ or $g_{0}$; in that case, a parabolic structure at $p$ that is invariant under the polar part of $\varphi$ corresponds to a complex line $\Psi \subset E_{p}$ that lies in either $\mathcal{L}$ or $\mathcal{L}^{-1}$, and $E$ is unstable. Conversely, if $\mathcal{L}$ is of order 2 , we can make a stable parabolic bundle $E$ that is an extension of $\mathcal{L}$ by $\mathcal{L}$ (a direct sum $\mathcal{L} \oplus \mathcal{L}$ is unstable with any parabolic structure), and it admits a nilpotent Higgs field $\varphi$ whose polar part leaves fixed the parabolic structure.

Here is a brief explanation, in the context of $S L_{2}$ on a curve $C$ of genus $g$, of the behavior we have just seen. (Ramification can be incorporated in the following argument by allowing certain poles in the Higgs field and the quadratic differentials.) Let $\varphi_{1}, \ldots, \varphi_{3 g-3}$ be a basis of $H^{0}(C, K \otimes \operatorname{ad}(E))$. So a general Higgs field is $\varphi=\sum_{i} a_{i} \varphi_{i}$, for some complex numbers $a_{i}$. The fiber $U_{E}$ of $T^{*} \mathcal{M}$ at the point corresponding to $E$ is thus parametrized by $a_{1}, \ldots, a_{3 g-3}$. Similarly, let $w_{1}, \ldots, w_{3 g-3}$ be a basis of $H^{0}\left(C, K^{2}\right)$. A fiber $\boldsymbol{F}$ of the Hitchin fibration is specified by $\operatorname{Tr} \varphi^{2}=\sum t_{i} w_{i}$, for some complex numbers $t_{i}$. Explicitly, this gives a set of $3 g-3$ quadratic equations

$$
P_{j}\left(a_{1}, \ldots, a_{3 g-3}\right)=t_{j}, \quad j=1, \ldots, 3 g-3 .
$$

Generically, such a set of equations has precisely $2^{3 g-3}$ solutions, weighted by multiplicity. These are the intersection points of the fiber $U_{E}$ with $\boldsymbol{F}$. If 
we add one more variable $a_{0}$ to make the equations homogeneous

$$
P_{j}\left(a_{1}, \ldots, a_{3 g-3}\right)=t_{j} a_{0}^{2}, \quad j=1, \ldots, 3 g-3,
$$

then the number of solutions in $\mathbb{C P}^{3 g-3}$, weighted by multiplicity, is always precisely $2^{3 g-3}$ if it is finite. The number of solutions of the affine equations (3.50) is less than this precisely if the homogeneous equations (3.51) have solutions with $a_{0}=0$. But solutions of the homogeneous equations with $a_{0}=0$ correspond to Higgs fields $\varphi$ with $\operatorname{Tr} \varphi^{2}=0$, that is, to nilpotent Higgs fields. Thus, some of the intersections of $U_{E}$ with $\boldsymbol{F}$ go to infinity precisely when $E$ admits a nonzero nilpotent Higgs field, just as we found in our example. (The example involves a ramified case in which the polynomials $P_{j}$ in Equation (3.50) are not homogeneous, so that the homogeneous form of Equation (3.51) is slightly different.)

3.9.3. Comparison of the two components What we have learned makes it possible to find an explicit mapping between the two components of $\mathcal{M}_{\mathrm{H}}$, as predicted in Section 3.6.2.

We want to explicitly map between the description of $\mathcal{M}_{\mathrm{H}}\left(S L_{2}\right)$ given by the familiar equation $P=0$, where

$$
P=\rho^{2}+(2 u+w) f(u)-\frac{f^{\prime}(u)^{2}}{4}
$$

and its analog

$$
b_{1}^{2}+b_{2}^{2}+b_{3}^{2}=1
$$

for the improper component $\mathcal{M}_{\mathrm{H}}\left(S L_{2}^{*}\right)$.

We already know from Section 3.15 that the change of variables we want preserves $w$, which for the improper component is

$$
w=e_{1} b_{1}^{2}+e_{2} b_{2}^{2}+e_{3} b_{3}^{2} .
$$

In addition, the desired change of variables commutes with the projection to $\mathcal{M}$ of a Zariski open set in $\mathcal{M}_{\mathrm{H}}$. The reason for this is that the $\varphi$-invariant Hecke modification of a bundle $E$ that was used in Section 3.6.2 depends only on the parabolic structure on $E$ that is determined by $\varphi$, and not on $\varphi$ itself. So it commutes with the projection from $\mathcal{M}_{\mathrm{H}}^{0}$ to $\mathcal{M}$, the moduli space of stable parabolic bundles.

For the proper component, $\mathcal{M}$ is parametrized by the variable $u$, and for the improper component, it is similarly parametrized by 
$z=\left(1-b_{1}\right) /\left(b_{2}-i b_{3}\right)$. These parametrizations are unique up to a fractional linear transformation, so $z$ must map to $(a u+b) /(c u+d)$ for some constants $a, b, c$ and $d$. The constants can be determined from the fact that the condition for a parabolic bundle $E$ to admit a nilpotent Higgs field (with a possible pole at $p$ whose residue preserves the parabolic structure) is also preserved by the map between the two components. Indeed, that map preserves both the Hitchin fibration and the projection $\mathcal{M}_{\mathrm{H}}^{0} \rightarrow \mathcal{M}$, so it maps missing points on Hitchin fibers to missing points on Hitchin fibers.

So the map between the two components identifies the four points $u=e_{1}, e_{2}, e_{3}, \infty$ with the four values of $z$ given in Equation (3.45). Up to the action of $Q$, this determines the relation between $z$ and $u$ to be

$$
u=\frac{e_{2} \sqrt{e_{3}-e_{1}}+e_{3} \sqrt{e_{2}-e_{1}}+\left(e_{1}-\sqrt{e_{3}-e_{1}} \sqrt{e_{2}-e_{1}}\right) \sqrt{e_{2}-e_{3}} z}{\sqrt{e_{3}-e_{1}}+\sqrt{e_{2}-e_{1}}+\sqrt{e_{2}-e_{3}} z} .
$$

$\mathcal{M}_{\mathrm{H}}\left(S L_{2}\right)$ is a double cover of the $w-u$ plane given by the quadratic equation (3.52) for $\rho$, and $\mathcal{M}_{\mathrm{H}}\left(S L_{2}^{*}\right)$ is similarly a double cover of the $w-z$ plane given by the quadratic equation (3.46) for $\widetilde{v}$. An elementary computation shows that the two quadratic equations are equivalent under the change of variables

$$
\widetilde{v}=\rho \frac{d u}{d z} \frac{1}{f(u)}-\frac{1}{2 A} \frac{d A}{d z}
$$

where $A$ was defined in Equation (3.47).

This gives the map between the two spaces. Finally, it is straightforward to verify that the complex symplectic form of $\mathcal{M}_{\mathrm{H}}\left(S L_{2}^{*}\right)$, given in Equation (3.42), maps to the complex symplectic form of $\mathcal{M}_{\mathrm{H}}\left(S L_{2}\right)$, which is similarly given by

$$
\Omega=\sigma_{0} \frac{d u \wedge d \rho}{f(u)}=\sigma_{0} \frac{d u \wedge d \rho}{\partial P / \partial w} .
$$

Because of this isomorphism, in comparing $A$-branes to $\mathcal{D}$-modules, as we do in Section 4, it suffices to consider the improper component. Whatever we can learn about $A$-branes and their corresponding $\mathcal{D}$-modules from the geometry of $\mathcal{M}_{\mathrm{H}}$ will be the same for the two components.

\subsection{Mirror symmetry of orbifolds}

Here we will briefly place some of the issues that we have considered in a more general context of mirror symmetry. 
First of all, let us note the following simple fact about the classical geometry. $\mathcal{M}_{\mathrm{H}}\left(S_{3}\right)$ has an $A_{1}$ singularity contained in a fiber that consists of a single $\mathbb{P}^{1}$ intersecting at two points. (There are three such singular fibers, but we consider here just one of them.) If we blow up the $A_{1}$ singularity, we eliminate the singularity (of the total space) and create a second $\mathbb{P}^{1}$. The blowup creates a new elliptic fibration with a singular fiber that now contains two $\mathbb{P}^{1}$ 's, each intersecting at two points. The total space is now smooth, but has a singular fiber as just described. This is precisely the geometry of $\mathcal{M}_{\mathrm{H}}\left(S L_{2}\right)$ near its special fibers.

The conclusion therefore is that if we change what physicists would call the "Kahler modulus" by this blowup, we could convert the local geometry for $\mathrm{SO}_{3}$ to that for $\mathrm{SL}_{2}$. Let us think about this local geometry more systematically. From a hyper-Kahler point of view, the deformation/resolution of an $A_{1}$ orbifold singularity is controlled by three real parameters $\vec{\mu}$. If one picks one of the complex structures - it is natural to select the complex structure $I$ in which the Hitchin fibration is holomorphic - then $\vec{\mu}$ splits up as a real parameter $\mu_{\mathbb{R}}$ that controls the Kahler class and a complex parameter $\mu_{\mathbb{C}}$ that controls the complex structure. Varieties with the same $\mu_{\mathbb{C}}$ and different $\mu_{\mathbb{R}}$ are birationally equivalent. A singularity occurs only if $\vec{\mu}=0$.

In the context of two-dimensional sigma models, there is an additional parameter, a theta-like angle which in [30, section 6.2], where this geometry is reviewed, is called $\eta$; it takes values in $\mathbb{R} / \mathbb{Z}$. From the point of view of one of the complex structures, say $I, \eta$ combines with $\mu_{\mathbb{R}}$ to a second complex parameter $\mu_{\mathbb{C}}^{\prime}=\mu_{\mathbb{R}}+i \eta$. This parameter controls the complexified Kahler class associated with the singularity, while $\mu_{\mathbb{C}}$ controls the complex structure.

The sigma model is singular if and only if $\vec{\mu}=\eta=0$ or equivalently $\mu_{\mathbb{C}}=$ $\mu_{\mathbb{C}}^{\prime}=0$. Having a singularity of the sigma model is an intrinsic condition and hence is invariant under any duality. In the context of Type IIA superstring theory, the singularity of the sigma model at $\vec{\mu}=\eta=0$ leads to an $S U(2)$ or $A_{1}$ gauge symmetry in spacetime; this is possibly the most satisfying statement of the relation of the group $A_{1}$ to the $A_{1}$ singularity.

In geometric Langlands, we want to treat the $\mathbb{R}^{4} / \mathbb{Z}_{2}$ singularity in the sigma model of $\mathcal{M}_{\mathrm{H}}\left(\mathrm{SO}_{3}\right)$ as an orbifold point. The sigma model can be treated as an orbifold precisely [6] if $\vec{\mu}=0, \eta=1 / 2$. At this value, we have $\mu_{\mathbb{C}}=0, \mu_{\mathbb{C}}^{\prime} \neq 0$. This means that the deformation away from the point $\mu_{\mathbb{C}}=\mu_{\mathbb{C}}^{\prime}=0$ is best understood as a resolution, not a deformation, though it is a resolution in a nonclassical sense, involving $\eta=\operatorname{Im} \mu_{\mathbb{C}}^{\prime}$ rather than $\mu_{\mathbb{R}}=\operatorname{Re} \mu_{\mathbb{C}}^{\prime}$. In contrast, to get to the local geometry of $\mathcal{M}_{\mathrm{H}}\left(S L_{2}\right)$, 
we must make, as we explained at the outset, a classical resolution, with $\operatorname{Re} \mu_{\mathbb{C}}^{\prime} \neq 0$.

Thus, in this situation, the $T$-duality on the fibers of the Hitchin fibration exchanges a nonclassical resolution involving $\operatorname{Im} \mu_{\mathbb{C}}^{\prime}$ with a classical resolution involving $\operatorname{Re} \mu_{\mathbb{C}}^{\prime}$. This is consistent with the following: as the Hitchin fibration is holomorphic in complex structure $I, T$-duality on the fibers of this fibration map complex parameters (such as $\mu_{\mathbb{C}}$ ) to complex parameters and Kahler parameters (such as $\mu_{\mathbb{C}}^{\prime}$ ) to Kahler parameters.

A last comment is that in Section 5.2, we will encounter an analog of all this involving a singularity $\mathbb{C}^{2 n} / \mathbb{Z}_{2}, n>1$. In this case, in classical geometry, the singularity can be neither deformed nor resolved, and in the sigma model, it can only be treated as an orbifold. Thus there is no close analog of the above discussion.

\subsection{Relation to Seiberg-Witten theory}

The Hitchin fibrations that we have considered here are very similar to elliptic fibrations that appear in Seiberg-Witten theory. For example, modulo some elementary changes of variable, Equation (3.39) for the $\mathrm{SO}_{3}$ moduli space coincides with [71, Equation (16.24)]. The latter equation describes the Seiberg-Witten fibration for the four-dimensional $\mathcal{N}=2^{*}$ theory $^{21}$ with gauge group $S U(2)$. The same singular fibers of the Hitchin fibration that we are relating here to endoscopy also play a central role in Seiberg-Witten theory. Physically, they describe the appearance of massless charged hypermultiplets; mathematically, they give the main contribution in the application of Seiberg-Witten theory to four-manifolds.

The Seiberg-Witten fibration of the $\mathcal{N}=2^{*}$ theory was not originally understood as a Hitchin fibration, but this was later done [19] as a step toward understanding the $\mathcal{N}=2^{*}$ theory for $S U(N)$.

The fact that the same elliptic fibration appears in two different problems can be explained by reducing the six-dimensional world-volume of a certain $M$-theory five-brane from six to four dimensions in two different ways. We consider $M$-theory on the 11-manifold $X=\mathbb{R}^{2} \times \mathbb{T}^{2} \times T^{*} C \times \mathbb{R}^{3}$, where $C$ is an elliptic curve. We let $D \subset T^{*} C$ be a spectral curve ${ }^{22}$ associated with an $S L_{2}$ Higgs bundle on $C$ with one point of ramification (for some value of $w$ ).

\footnotetext{
${ }^{21}$ This is the $\mathcal{N}=2$ theory with a massive hypermultiplet in the adjoint representation. As the hypermultiplet mass becomes large, this theory can reduce to the minimal $\mathcal{N}=2$ theory, which is the basic case of Seiberg-Witten theory.

${ }^{22}$ This notion is reviewed in Section 4.1.
} 
And we consider an $M$-theory five-brane whose world-volume is $\mathbb{R}^{2} \times \mathbb{T}^{2} \times$ $D$. There are two ways to look at this situation, depending on whether $\mathbb{T}^{2}$ or $C$ is smaller.

(1) If $\mathbb{T}^{2}$ is smaller, the first step is to consider compactification of the five-brane on $\mathbb{T}^{2}$. This leads to $\mathcal{N}=4$ super Yang-Mills theory with gauge group $U(2)$ (because the projection $D \rightarrow C$ is generically a double cover, the gauge group is $U(2)$, or effectively $S U(2)$, since the center plays little role). Geometric Langlands is associated with subsequent compactification to two dimensions on $C$; the particular curve $D$ describes the behavior in the presence of a surface operator associated with ramification, as discussed in [30, Section 6.4].

(2) If $C$ is smaller, we consider first the compactification on $C$, which [80] leads to the $\mathcal{N}=2^{*}$ theory on $\mathbb{R}^{2} \times \mathbb{T}^{2}$. The elliptic fibration (3.39) describes this theory on $\mathbb{R}^{4}$. The result of further compactification from $\mathbb{R}^{4}$ to $\mathbb{R}^{2} \times \mathbb{T}^{2}$ is that the elliptic fibration must be endowed with a hyper-Kahler metric [72].

\section{A-branes and $\mathcal{D}$-modules}

As promised in Section 2.3.1, we will give more details on the relation between an $A$-brane on $\mathcal{M}_{\mathrm{H}}$ and the corresponding twisted $\mathcal{D}$-module on $\mathcal{M}$. The main goal is to explain the significance of Equation (3.49) that describes the behavior of a Hitchin fiber restricted to $\widetilde{T}^{*} \mathcal{M}$ near its "missing" divisors (which in that example are points, $\operatorname{since} \operatorname{dim} \mathcal{M}=1$ ).

For this, we need the theory of spectral curves, which will also be a primary tool in Section 5. So we begin with a very brief sketch of this theory.

Although the arguments in [39, Section 11] relating $A$-branes to twisted $\mathcal{D}$-modules are fairly clear from a physical point of view, they are completely conjectural mathematically. The following discussion involves an extra layer of conjecture, since although what we will describe is qualitatively in accord with what one would expect from [39], it is not yet based on equally clear-cut physics arguments.

For other approaches to these questions, see $[59,61,62]$.

\subsection{Spectral curves}

The idea is most simply explained for $G L_{n}$ or $S L_{n}$. Let $(E, \varphi)$ be a Higgs bundle, where $E \rightarrow C$ is a vector bundle of rank $n$ and $\varphi \in H^{0}(C, K \otimes$ 
$\operatorname{ad}(E))$ is the Higgs field. Associated to these data, one defines a curve $D$ in the cotangent bundle $T^{*} C$ as follows. Letting $z$ denote a linear function on the fibers of the cotangent bundle, the curve $D$, which is known as the spectral curve, is defined by the equation

$$
\operatorname{det}(z-\varphi)=0
$$

The projection $\pi: T^{*} C \rightarrow C$ restricts to an $n$-fold cover $\pi: D \rightarrow C$.

Generically, $D$ is smooth and irreducible and has genus $g^{\prime}=n^{2} g-n^{2}+1$, where $g$ is the genus of $C$. For ordinary Higgs bundles, $D \subset T^{*} C$ is a complete projective curve, but for ramified Higgs bundles, this is not so as $\varphi$ has poles. In that case, one completes $D$ to a projective curve by adding a few points that are not in $T^{*} C$.

One defines a line bundle $\mathcal{L} \rightarrow D$ as the cokernel of the map $\left(\pi^{*}(\varphi)-\right.$ $z): \pi^{*}(E) \rightarrow \pi^{*}(E) \otimes K$. To be more precise, $\mathcal{L}$ is a line bundle when $D$ is smooth but in general may be a torsion-free sheaf.

So to the Higgs bundle $(E, \varphi)$ on $C$, we associate the following data: the spectral curve $\pi: D \rightarrow C$ and the line bundle or torsion-free sheaf $\mathcal{L} \rightarrow D$. From these data, it is possible to reconstruct the original Higgs bundle. One recovers $E$ by $E=\pi_{*}(\mathcal{L})$. And the Higgs field $\varphi: E \rightarrow E \otimes K$ can similarly be recovered from $\pi: D \rightarrow C$ as $\pi_{*}(z)$, where here multiplication by $z$ is understood as a map from $\mathcal{L}$ to $\mathcal{L} \otimes \pi^{*}\left(T^{*} C\right)$. In this way, the Higgs bundle $(E, \varphi)$ can be recovered from the pair $(D, \mathcal{L})$.

In this description, the base $\boldsymbol{B}$ of the Hitchin fibration parametrizes the possible spectral curves $D$. For example, for $G=S L_{2}$, the equation defining $D$ reduces to $z^{2}+\operatorname{det} \varphi=0$, so $\boldsymbol{B}$ is parametrized by the quadratic differential $\operatorname{det} \varphi=-\operatorname{Tr} \varphi^{2} / 2$. The Hitchin fibration is the map that takes a pair $(D, \mathcal{L})$ to $D$. The fiber $\boldsymbol{F}$ of the Hitchin fibration is parametrized by the possible choices of $\mathcal{L}$ for a given spectral curve $D$.

What line bundles $\mathcal{L} \rightarrow D$ arise in this construction? For $G L_{n}$, if we allow all Higgs bundles $(E, \varphi)$, with no restriction on $\operatorname{det} E$, then all $\mathcal{L} \rightarrow D$ can arise. Thus, $\mathcal{L}$ is associated with an arbitrary point in $\operatorname{Pic} D$. If we wish to associate $(E, \varphi)$ to a local system by solving Hitchin's equations, then $\operatorname{det} E$ must have degree zero and we must restrict $\mathcal{L}$ to a particular component of Pic $D$. A small computation with Riemann-Roch shows that in order for $E=\pi_{*}(\mathcal{L})$ to be of degree zero, the degree of $\mathcal{L}$ must be

$$
\operatorname{deg} \mathcal{L}=n(n-1)(g-1) .
$$

(See Section 4.9 for a version of this computation.) 
For $S L_{n}$, we must further restrict $\mathcal{L}$ so that $\operatorname{det} E$ is trivial, not just of degree zero.

\subsection{Relation to $A$-branes}

Condition (4.2) on the degree of $\mathcal{L}$ can be usefully stated as follows. Let $K_{C}^{1 / 2}$ and $K_{D}^{1 / 2}$ denote the square roots of the canonical bundles of $C$ and $D$, respectively. Then $\mathcal{L}$ has the form $\mathcal{L}=\pi^{*}\left(K_{C}^{-1 / 2}\right) \otimes K_{D}^{1 / 2} \otimes \mathcal{N}$, where $\mathcal{N} \rightarrow D$ has degree 0 . An evocative way to restate this is to say that we can define $E$ by

$$
K_{C}^{1 / 2} \otimes E=\pi_{*}\left(K_{D}^{1 / 2} \otimes \mathcal{N}\right)
$$

The virtue of this last description is that it is closely related to the theory of $A$-branes. Let $X$ be a symplectic manifold and $L \subset X$ a Lagrangian submanifold. We begin with the general case of a real symplectic manifold with a real Lagrangian submanifold. To define an $A$-brane $\mathcal{B}$ supported on $L$, we choose roughly speaking a flat vector bundle $V \rightarrow L$ and a spin bundle $\mathcal{W}$ on $L$. Then the brane $\mathcal{B}$ is associated in differential geometry with the vector bundle $\mathcal{W} \otimes V \rightarrow L$.

Now specialize to the case that $X$ is a complex symplectic manifold and $L$ a complex Lagrangian submanifold of complex dimension $d$. Then, writing $K_{L}^{1 / 2}$ for a square root of the canonical bundle of $L$ and $\Omega^{0, i}$ for the sheaf of $(0, i)$-forms on $L$, a spin bundle of $L$ takes the form $\mathcal{W}=K_{L}^{1 / 2} \otimes$ $\left(\oplus_{i=0}^{d} \Omega^{0, i}\right)$. As a result, in algebraic geometry, one associates $\mathcal{B}$ with the sheaf of sections of $K_{L}^{1 / 2} \otimes V \rightarrow L$; the tensor product with $\oplus_{i=0}^{d} \Omega^{0, i}$ simply supplies the $\bar{\partial}$ resolution of this sheaf. So in Equation (4.3), the right-hand side involves precisely the defining data of an $A$-brane supported on the Lagrangian submanifold $D$, that is, the line bundle $K_{D}^{1 / 2} \otimes \mathcal{N}$. We will see shortly in what sense the left-hand side is also natural.

Remark 4.1. A more intrinsic formulation avoids choosing spin bundles over $D$ and $L$. In the most precise description, $V \rightarrow L$ is not a flat vector bundle, but a flat twisted vector bundle. The twisting is by a complex gerbe $\mathcal{G}$ of order 2 whose local trivializations correspond to spin structures on the normal bundle to $L$ in $X$ ( $\mathcal{G}$ is obtained from a $\mathbb{Z}_{2}$ gerbe via the embedding $\left.\{ \pm 1\} \subset \mathbb{C}^{\times}\right)$. The twisting by $\mathcal{G}$ is related to the fact that branes in $X$ actually have an interpretation in terms of the $K$-theory of $X$. For example, see [24]. The key point is that a submanifold $L \subset X$ equipped with a 
G-twisted vector bundle defines a class in $K(X)$, while $L$ equipped with an ordinary vector bundle does not define such a class.

The gerbe $\mathcal{G}$ will also appear in Section 10. Except in that section, we will not incorporate this extra layer of subtlety. Our main example of an $A$-brane in most of the paper is a brane supported on a fiber $\boldsymbol{F}$ of the Hitchin fibration. In this case, the determinant of the normal bundle is trivial and has a global square root that is also trivial, giving a canonical trivialization of $\mathcal{G}$.

A rank 1 bundle twisted by $\mathcal{G}$ determines what is called a $\operatorname{Spin}_{c}$ struc- $^{-}$ ture. A flat rank $1 \mathcal{G}$-twisted bundle corresponds to a flat $\mathrm{Spin}_{c}$ structure. In our present example that $L$ is a curve $D$, a line bundle of the form $K_{D}^{1 / 2} \otimes \mathcal{N} \rightarrow D$, where $\mathcal{N}$ has degree 0 , corresponds naturally to a flat $\mathrm{Spin}_{c}$ structure. That is an intrinsic way to characterize line bundles of the form $K_{D}^{1 / 2} \otimes \mathcal{N}$ without introducing separately either factor in the tensor product.

\subsection{Map from $A$-branes to twisted $\mathcal{D}$-modules}

Now we can explain the basic strategy for mapping $A$-branes to twisted $\mathcal{D}$-modules. We do this initially for $A$-branes in a complex symplectic manifold $X=T^{*} Y$ for the case that $Y$ is a curve.

We let $D \subset X$ be any smooth curve such that the projection $\pi: X \rightarrow Y$ restricts to a finite cover $\pi: D \rightarrow Y$. In Section 4.1, such a curve $D$ arose as the spectral curve of a Higgs bundle $(E, \varphi)$ over $C$. Though this is the motivating example, we have given the base curve a new name $Y$ because in our application, $Y$ will be in fact not the original curve $C$ but a moduli space $\mathcal{M}$ of stable parabolic bundles over $C$.

For dimensional reasons, $D$ is a Lagrangian submanifold with respect to the holomorphic symplectic structure of $X$. We define a rank $1 A$-brane supported on $D$ by picking a flat $\operatorname{Spin}_{c}$ structure on $D$ that we write not quite intrinsically as a choice of line bundle $K_{D}^{1 / 2} \otimes \mathcal{N} \rightarrow D$, where $\mathcal{N} \rightarrow D$ is a line bundle of degree 0 .

Then, roughly speaking, according to Equation (4.3), we define a vector bundle $E \rightarrow Y$ by $K_{Y}^{1 / 2} \otimes E=\pi_{*}\left(K_{D}^{1 / 2} \otimes \mathcal{N}\right)$. And we define a Higgs field $\varphi$ by $\varphi=\pi_{*}(z)$. So $(E, \varphi)$ is a Higgs bundle over $Y$.

The key step comes next. By solving Hitchin's equations, we relate the Higgs bundle $(E, \varphi)$ to a rank $n$ local system over $Y$ that we also call $E$. Hence instead of just thinking of $K_{Y}^{1 / 2} \otimes E$ as a vector bundle, we can think 
of its sheaf of sections as a twisted $\mathcal{D}$-module over $Y$, that is, a sheaf of modules for the sheaf $\mathcal{D}^{*}$ of differential operators acting on sections of $K_{Y}^{1 / 2}$.

An important detail here is that starting with an $A$-brane defined by a flat $\operatorname{Spin}_{c}$ bundle that we write loosely as $K_{D}^{1 / 2} \otimes \mathcal{N}$, we do not quite get intrinsically a degree 0 vector bundle $E \rightarrow Y$; rather we get a vector bundle of nonzero degree that we have written nonintrinsically as $K_{Y}^{1 / 2} \otimes E$. There is no canonical choice of $K_{Y}^{1 / 2}$, so there is also no canonical way to define $E$. Only the tensor product of the two is canonical, and this is why an $A$ brane in $T^{*} Y$ supported on the curve $D$ maps canonically not to an ordinary $\mathcal{D}$-module over $Y$ (such as the sheaf of sections of a local system $E$ ) but to a twisted $\mathcal{D}$-module (the sheaf of sections of $K_{Y}^{1 / 2} \otimes E$ ).

Though $E$ is only defined up to a twist by a line bundle of order 2, this twist does not affect Hitchin's equations, and the twisted $\mathcal{D}$-module that emerges from the construction depends only on the original $A$-brane, and not on any other choice. What is not quite canonical is to describe this twisted $\mathcal{D}$-module via a tensor product $K_{Y}^{1 / 2} \otimes E$.

\subsection{Poles}

For application to geometric Langlands, it is essential to extend this to the case that the curve $D \subset T^{*} Y$ is not compact and the projection $\pi: D \rightarrow Y$ is only generically an $n$-fold cover. As in Equation (2.6), we suppose that locally, near some point $u_{0} \in Y$, some branches of $\pi: D \rightarrow Y$ go to infinity. We let $u$ denote a local coordinate on $Y$ near $u_{0}$, and pick a second coordinate $s$ on $X=T^{*} Y$ so that the symplectic structure is $d u \wedge d s$. In the most basic case, we assume that the $n$ branches are described by functions $s_{i}(u)$ whose singularities at $u=u_{0}$ are simple poles. Thus,

$$
s_{i}(u) \sim \frac{c_{i}}{u-u_{0}}, \quad i=1, \ldots, n
$$

with complex constants $c_{i}$, some of which may vanish.

Let $\mathcal{U}$ be a small neighborhood of the point $u_{0} \in Y$ and trivialize $\mathcal{L}$ over $\pi^{-1}(\mathcal{U})$. Then $E=\pi_{*}(\mathcal{L})$ is naturally trivialized over $\mathcal{U}$ and relative to this trivialization, the Higgs field $\varphi$ is diagonal, $\varphi=\operatorname{diag}\left(\varphi_{1}, \ldots, \varphi_{n}\right)$, where the $\varphi_{i}$ are differentials with poles at $u=u_{0}$ :

$$
\varphi_{i} \sim c_{i} \frac{d u}{u-u_{0}} .
$$

Just as in the case that $D$ is compact, an $A$-brane supported on $D$ still gives rise to a Higgs bundle $(E, \varphi)$ over $Y$ (twisted as above by $K_{Y}^{1 / 2}$ ). 
However, $\varphi$ now has a simple pole at the points in $Y$, such as $u_{0}$, at which $\pi: D \rightarrow Y$ fails to be an $n$-fold cover.

We can still solve Hitchin's equations and associate to this data a (twisted) local system, since Hitchin's equations work nicely in the presence of prescribed poles [68]. We have already described in Equation (3.4) the appropriate polar behavior of the fields entering in Hitchin's equations. But now we write $\alpha^{*}, \beta^{*}$ and $\gamma^{*}$ for the parameters characterizing a ramified Higgs bundle on the curve $Y$ :

$$
\begin{aligned}
& A=\alpha^{*} d \theta+\cdots \\
& \phi=\beta^{*} \frac{d r}{r}-\gamma^{*} d \theta+\cdots
\end{aligned}
$$

(Here $u-u_{0}=r e^{i \theta}$.) In the rank $n$ case, the parameters $\alpha^{*}, \beta^{*}$ and $\gamma^{*}$ take values in the Lie algebra of a maximal torus in the compact form of $G L_{n}$; thus, they are imaginary ${ }^{23}$ diagonal matrices.

We reserve the names ${ }^{\mathrm{L}} \alpha,{ }^{\mathrm{L}} \beta$ and ${ }^{\mathrm{L}} \gamma$ for the ramification parameters on $C$ of the original ${ }^{\mathrm{L}} G$ local system, and $\alpha, \beta$ and $\gamma$ for their counterparts in the $A$-model. Until this point, we have not distinguished in the notation between the ramification parameters for ${ }^{\mathrm{L}} G$ and $G$. This is because we have been discussing classical geometry, and the same facts are relevant to both the $A$-model and the $B$-model. Henceforth, it will help to be more precise.

As explained in Section 2.1.1, a solution of Hitchin's equations with a singularity as in Equation (4.6) determines, depending on how we look at it, either a Higgs bundle with a singularity at $u_{0}$ or a local system with such a singularity. The Higgs bundle has a pole

$$
\varphi \sim \sigma^{*} \frac{d u}{u-u_{0}}, \quad \sigma^{*}=\frac{\beta^{*}+i \gamma^{*}}{2} .
$$

The local system has monodromy

$$
M^{*}=\exp \left(-2 \pi\left(\alpha^{*}-i \gamma^{*}\right)\right)
$$

It is useful to set $\sigma^{*}=\operatorname{diag}\left(\sigma_{1}^{*}, \ldots, \sigma_{n}^{*}\right)$ and $\sigma_{i}^{*}=\frac{1}{2}\left(\beta_{i}^{*}+i \gamma_{i}^{*}\right)$. Comparing Equations (4.5) and (4.7), we see that

$$
\sigma_{i}^{*}=c_{i}
$$

\footnotetext{
${ }^{23}$ The elements of the real Lie algebra of a compact group acts as anti-Hermitian
} matrices in a unitary representation. 
or in other words

$$
\beta_{i}^{*}+i \gamma_{i}^{*}=2 c_{i} .
$$

Thus, given a curve $D \subset T^{*} Y$ with poles of the type we have considered, $\beta^{*}$ and $\gamma^{*}$ are determined by the geometry. The eigenvalues of the monodromy $M^{*}$ have absolute values $\exp \left(2 \pi i \gamma_{i}^{*}\right)$, which are determined by $D$. The arguments of the eigenvalues depend on $\alpha_{i}^{*}$. The geometry of $D$ does not determine $\alpha_{i}^{*}$; we can solve Hitchin's equations and get a twisted $\mathcal{D}$-module over $Y$ for any choice at all of $\alpha_{i}^{*}$.

\subsection{Application to our example}

We can get some insight about $\alpha^{*}$ by considering the familiar example - the geometric Langlands program for $S L_{2}$ on a curve $C$ of genus 1 with 1 point of ramification. In this problem, we do not expect to be able to determine $\alpha^{*}$ uniquely from the data considered so far; it is perfectly natural that it should also depend on the underlying parameter ${ }^{\mathrm{L}} \alpha$ of the original ${ }^{\mathrm{L}} G$ local system. A physics-based method to determine $\alpha^{*}$ in this problem is not presently available, but considerable information comes from very simple arguments. (A conjectural description of $\alpha^{*}$ is given at the end of Section 4.7.)

The moduli space of parabolic $S L_{2}$ bundles over $C$ in this situation is a curve $\mathcal{M}$ of genus zero, as we have described. We take the curve $Y$ in the above discussion to be $\mathcal{M}$, and we take $D$ to be a fiber $\boldsymbol{F}$ of the Hitchin fibration, regarded as a curve in $\widetilde{T}^{*} Y \subset \mathcal{M}_{\mathrm{H}}$. In considering this example, we are jumping ahead of our story slightly, because here $\widetilde{T}^{*} Y$ is an affine deformation of the cotangent bundle of $Y$, while in Section 4.4, we considered a curve in the undeformed cotangent bundle. For the moment we overlook this difference, which will be incorporated in Section 4.6.

There are two ramified Higgs bundles in this example of the geometric Langlands program. The input is a ramified Higgs bundle $(E, \varphi)$ over the curve $C$, with parameters ${ }^{\mathrm{L}} \alpha,{ }^{\mathrm{L}} \beta$ and ${ }^{\mathrm{L}} \gamma$. The output is a ramified Higgs bundle over the curve $Y=\mathcal{M}$, with parameters $\alpha^{*}, \beta^{*}$ and $\gamma^{*}$. We would like to determine the output parameters as a function of the input parameters. (The output parameters may a priori be different at different points on $\mathcal{M}$ where the induced local system is singular.)

In geometric Langlands, one views the input Higgs bundle as determining a local system over $C$ with monodromy $M=\exp \left(-2 \pi\left({ }^{\mathrm{L}} \alpha-i^{\mathrm{L}} \gamma\right)\right)$. The output is a twisted $\mathcal{D}$-module over $\mathcal{M}$, represented by a local system with monodromy $M^{*}=\exp \left(-2 \pi\left(\alpha^{*}-i \gamma^{*}\right)\right)$. From this point of view, it is clear 
that if there is a geometric Langlands duality, then $\alpha^{*}-i \gamma^{*}$ varies holomorphically in ${ }^{\mathrm{L}} \alpha-i^{\mathrm{L}} \gamma$ and is independent of ${ }^{\mathrm{L}} \beta$. The last statement holds because the input local system does not depend on ${ }^{\mathrm{L}} \beta$, though its associated Higgs bundle does. So in particular, we have

$$
\frac{\partial \gamma^{*}}{\partial^{\mathrm{L}} \beta}=0
$$

A priori, it is not clear that $\alpha^{*}-i \gamma^{*}$ is a function only of ${ }^{\mathrm{L}} \alpha-i^{\mathrm{L}} \gamma$ (and not of the modulus of the input local system over $C$ ), but in a moment it will be clear that in our example, this is true for $\gamma^{*}$ and therefore by holomorphy also for $\alpha^{*}$.

Alternatively, Hitchin's equations identify the input local system with a Higgs bundle $(E, \varphi)$ and here the natural holomorphic parameter is ${ }^{\mathrm{L}} \sigma=$ $\left({ }^{\mathrm{L}} \beta+i^{\mathrm{L}} \gamma\right) / 2$. Duality maps this to a multiple of $\sigma=(\beta+i \gamma) / 2$, on which $\mathcal{M}_{\mathrm{H}}(G)$ depends holomorphically. So $\sigma^{*}=\left(\beta^{*}+i \gamma^{*}\right) / 2$ is holomorphic in ${ }^{\mathrm{L}} \sigma$ and is independent of ${ }^{\mathrm{L}} \alpha$; in particular,

$$
\frac{\partial \gamma^{*}}{\partial^{\mathrm{L}} \alpha}=0 \text {. }
$$

Taken together, the statements in the last two paragraphs mean that the output triple $\left(\alpha^{*}, \beta^{*}, \gamma^{*}\right)$ is a linear function of the input triple $\left({ }^{\mathrm{L}} \alpha,{ }^{\mathrm{L}} \beta,{ }^{\mathrm{L}} \gamma\right)$. One way to argue this begins with the Cauchy-Riemann equations asserting that $\beta^{*}+i \gamma^{*}$ is holomorphic in ${ }^{\mathrm{L}} \beta+i^{\mathrm{L}} \gamma$ :

$$
\begin{aligned}
& \frac{\partial \beta^{*}}{\partial^{\mathrm{L}} \beta}=\frac{\partial \gamma^{*}}{\partial^{\mathrm{L}} \gamma} \\
& \frac{\partial \gamma^{*}}{\partial^{\mathrm{L}} \beta}=-\frac{\partial \beta^{*}}{\partial^{\mathrm{L}} \gamma} .
\end{aligned}
$$

Using this and Equation (4.11), we see that $\partial^{2} \gamma^{*} / \partial^{\mathrm{L}} \gamma^{2}=\partial^{2} \beta^{*} / \partial^{\mathrm{L}} \beta \partial^{\mathrm{L}} \gamma=$ $-\partial^{2} \gamma^{*} / \partial^{\mathrm{L}} \beta^{2}=0$. Since $\gamma^{*}$ is independent of ${ }^{\mathrm{L}} \alpha$ and ${ }^{\mathrm{L}} \beta$, according to Equations (4.11) and (4.12), it follows that $\gamma^{*}=f\left({ }^{\mathrm{L}} \gamma\right)$, where $f$ is a possibly inhomogeneous linear function between the two Lie algebras. Then holomorphy of $\alpha^{*}-i \gamma^{*}$ in ${ }^{\mathrm{L}} \alpha-i^{\mathrm{L}} \gamma$ and of $\beta^{*}+i \gamma^{*}$ in ${ }^{\mathrm{L}} \beta+i^{\mathrm{L}} \gamma$ implies that

$$
\left(\alpha^{*}, \beta^{*}, \gamma^{*}\right)=\left(f\left({ }^{\mathrm{L}} \alpha\right)+d_{1}, f\left({ }^{\mathrm{L}} \beta\right)+d_{2}, f\left({ }^{\mathrm{L}} \gamma\right)\right)
$$

with some constants $d_{1}$ and $d_{2}$ and the same linear function $f$. In particular

$$
\sigma^{*}=f\left({ }^{\mathrm{L}} \sigma\right)+\frac{d_{2}}{2}
$$

must be a linear function of ${ }^{\mathrm{L}} \sigma$. 
We can compare this prediction of linearity to the results of Equations (3.49), where we computed $\sigma^{*}$ in our example. (As remarked in Section 3.9.1, we consider only the improper component, as the two are equivalent for these questions.) From Equation (3.49) we see that, up to permutation of the two eigenvalues of $\sigma^{*}$, we have

$$
\left(\sigma_{1}^{*}, \sigma_{2}^{*}\right)=\left(\sigma_{0}, 0\right)
$$

Here $\pm \sigma_{0}$ are the eigenvalues of ${ }^{\mathrm{L}} \sigma$. In particular, the linearity holds, and moreover the linear function $f$ is homogeneous and $d_{2}=0$. We would conjecture (without a solid physics-based argument) that also $d_{1}=0$, in which case $\varrho^{*}=\alpha^{*}-i \gamma^{*}$ is a homogeneous linear function of ${ }^{\mathrm{L}} \varrho={ }^{\mathrm{L}} \alpha-i^{\mathrm{L}} \gamma$. Then writing ${ }^{\mathrm{L}} \varrho=\operatorname{diag}\left({ }^{\mathrm{L}} \varrho_{0},-{ }^{\mathrm{L}} \varrho_{0}\right)$ ( $\varrho$ is traceless as it takes values in $\left.\mathfrak{s l}_{2}\right)$ and $\varrho^{*}=\left(\varrho_{1}^{*}, \varrho_{2}^{*}\right)$, Equation (4.16) implies that

$$
\left(\varrho_{1}^{*}, \varrho_{2}^{*}\right)=\left({ }^{\mathrm{L}} \varrho_{0}, 0\right)
$$

The Weyl symmetry between ${ }^{\mathrm{L}} \varrho_{0}$ and $-{ }^{\mathrm{L}} \varrho_{0}$ has been lost because in Section 3.9.1, we have chosen one of two possible maps $\mathcal{M}_{\mathrm{H}}^{0} \rightarrow \mathcal{M}$ as the starting point in associating a twisted $\mathcal{D}$-module on $\mathcal{M}$ to an $A$-brane on $\mathcal{M}_{\mathrm{H}}^{0}$.

Now go back to the ramified $S_{3}$ local system $E \rightarrow C \backslash p$ with semisimple monodromy around $p$. If we pick an extension over $p$ of the holomorphic (or topological) structure of $E$, then the monodromy $M$ of $E$ around $p$ makes sense as an element of $S L_{2}$ (not just $S O_{3}$ ), and is conjugate to $\operatorname{diag}\left(\lambda, \lambda^{-1}\right)$ with $\lambda=\exp \left(-2 \pi^{\mathrm{L}} \varrho\right)$. The analogous monodromy $M^{*}$ of the local system on $\mathcal{M}$ is conjugate to

$$
\operatorname{diag}\left(\exp \left(-2 \pi \varrho_{1}^{*}\right), \exp \left(-2 \pi \varrho_{2}^{*}\right)\right)
$$

So according to Equation (4.17), up to conjugacy the monodromies of the local system on $\mathcal{M}$ are

$$
M^{*}=\operatorname{diag}(\lambda, 1)
$$

What has been described so far applies to the generic case of an $A$-brane on $\mathcal{M}_{\mathrm{H}}$ supported on a smooth fiber $\boldsymbol{F}$ of the Hitchin fibration. Such an $A$-brane is associated with a rank 2 twisted local system on $\mathcal{M}$ with singularities at a few points, and the monodromies of the singularities are as just described. They are actually the same at each singularity.

For geometric endoscopy, we want the analogous description for the case that $\boldsymbol{F}$ is a union of two components $\boldsymbol{F}_{1}$ and $\boldsymbol{F}_{2}$. The fractional $A$-branes 
supported on just one component are associated with rank 1 twisted local systems over a Zariski open set in $\mathcal{M}$. It is explained in Remark 3.5 that each Lagrangian submanifold $\boldsymbol{F}_{1}$ or $\boldsymbol{F}_{2}$ has a simple pole analogous to that in Equation (3.49) at precisely two of the four points $z^{*}$. Hence the rank 1 local system on $\mathcal{M}$ corresponding to either of these branes has precisely 2 singularities, at each of which the monodromy is $\lambda$.

This may seem to entail a contradiction. The structure group of a rank 1 local system is the abelian group $G L_{1}$, so the product of its monodromies must equal 1, rather than $\lambda^{2}$ as we seem to get in the last paragraph. The resolution of this question entails a point that we have been omitting: $\mathcal{M}_{\mathrm{H}}^{0}$ is not the cotangent bundle of $\mathcal{M}$ but an affine deformation of this cotangent bundle. As we discuss next, this leads to a twist of the local system such that the product of monodromies at its singularities is central but is not simply equal to 1 .

\subsection{The central twist}

Our next task will be to understand $A$-branes in $X=\widetilde{T}^{*} Y$, where $X$ is an affine deformation of $T^{*} Y$. The deformation is classified by a class $\zeta \in$ $H^{1}\left(Y, \Omega^{1, c l}(Y)\right)$. Viewing $\zeta$ as a closed $(1,1)$-form, the integral $\int_{Y} \zeta / 2 \pi i$ is a complex number that we call $\zeta_{\mathbb{C}}$.

$A$-branes in $\widetilde{T}^{*} Y$ are related to twisted $\mathcal{D}$-modules on $Y$ that are twisted in a more interesting way than we have discussed so far in this paper. The relevant $\mathcal{D}$-modules are modules not for the sheaf of differential operators acting on $K_{Y}^{1 / 2}$, but for the sheaf of such operators acting on $K_{Y}^{1 / 2} \otimes \mathcal{T}_{\chi}$, where $\mathcal{T}_{\chi}$ is a "line bundle" of first Chern class $\chi$. ( $\chi$ is a class whose relation to $\zeta$ will be described.) $\mathcal{T}_{\chi}$ does not really exist as a line bundle unless the class $\chi$ is integral, but the sheaf $\mathcal{D}_{\chi}^{*}$ of differential operators acting on $K_{Y}^{1 / 2} \otimes \mathcal{T}_{\chi}$ does exist in any case.

In the ramified case of geometric Langlands, this can be deduced using representations of affine Kac-Moody algebras at the critical level $[26,27]$ or by analyzing the canonical coisotropic brane $\mathcal{A}_{c c}$, as in [30, Section 4.4]. Aiming to recover this result from the viewpoint adopted here, we let $D$ be a curve in $X$ such that the projection $\pi: X \rightarrow Y$ restricts to a map $\pi: D \rightarrow Y$ that is generically a finite cover. However, we allow $D$ to have "poles" over finitely many points in $Y$, as in Section 4.4. $D$ is also endowed with a suitable line bundle $\mathcal{L}$. It is instructive to first consider the case that $\pi: D \rightarrow Y$ is generically of degree 1 , so that $D$ is a section of $\pi: X \rightarrow Y$ over a Zariski open set in $Y$. In this case, there must be poles, if $\zeta \neq 0$. 
Otherwise, $D$ would be a global holomorphic section of $\pi$, whose existence would trivialize the class $\zeta$.

In the case that $X$ actually is globally the cotangent bundle $T^{*} Y$, the curve $D$ corresponds to a meromorphic differential on $X$. This differential is the Higgs field $\varphi$ of the rank 1 Higgs bundle that corresponds to $D$. At each point $u_{i} \in Y$ at which $\varphi$ has a pole, one can define its residue, called $\sigma_{i}^{*}$ in Equation (4.7). According to the residue theorem, the sum of residues vanishes:

$$
\sum_{i} \sigma_{i}^{*}=0
$$

When we use Hitchin's equations (which in this rank 1 case reduce to ordinary Hodge theory) to relate the pair $(D, \mathcal{L})$ to a rank 1 local system $E \rightarrow Y$, the absolute value of the monodromy at $u_{i}$ is $\exp \left(-2 \pi \operatorname{Im} \sigma_{i}^{*}\right)$. The vanishing of the sum of the residues maps to the fact that for a $G L_{1}$ local system, the product of these absolute values is 1 . (The phases of the monodromies are determined by $\alpha_{i}^{*}$.)

Now let us consider the case that $X=\widetilde{T}^{*} Y$ is actually an affine deformation of $T^{*} Y$. In this case, at each singular point $u_{i}$, we can still define a residue $\sigma_{i}^{*}$; indeed, the affine deformation is trivial locally, and though there is no canonical way to trivialize it, a change in the trivialization does not affect the residue. What is new in the case of an affine deformation is that the sum of residues no longer vanishes. Instead, we have

$$
\sum_{i} \sigma_{i}^{*}-\zeta_{\mathbb{C}}=0
$$

(One way to derive this result is to observe that we could trivialize $\zeta$ away from an arbitrarily chosen point $r \in Y$. Relative to this trivialization, $D$ has one more singular point, with residue $-\zeta_{\mathbb{C}}$, and Equation (4.20) is simply the vanishing of the sum of all residues.) The fact that the sum of the residues is a nonzero constant corresponds to the fact that the product of the absolute values of the monodromies is a constant not equal to 1 , in fact equal to $\exp \left(2 \pi \operatorname{Im} \zeta_{\mathbb{C}}\right)$.

This resolves the puzzle mentioned at the end of Section 4.5. In that computation, we used coordinates $z$ on $Y=\mathcal{M}$ and $v$ on the fiber of $\widetilde{T}^{*} \mathcal{M}$. The choice of these coordinates trivialized the affine deformation except at $z=\infty$. In particular, the deformation was trivialized near the poles of $D$ and hence did not affect the residues of those poles. With this trivialization, $D$ has an additional pole at $z=\infty$, and of course, the sum of all residues, including that last one, vanishes. 
In differential geometry, one can represent $\zeta$ as a closed ${ }^{24}(1,1)$-form and describe this situation as follows. A meromorphic section $D$ of $\pi: \widetilde{T}^{*} Y \rightarrow Y$ does not correspond to a meromorphic differential $\omega$. Rather it corresponds to a $(1,0)$-form on $Y$, with poles as above, and obeying

$$
\bar{\partial} \varphi=\zeta
$$

Integration of this formula over $C$ leads back to Equation (4.20); one integrates the left-hand side using the poles of $\varphi$ and expresses the integral of the right-hand side in terms of $\zeta_{\mathbb{C}}$.

Now let us consider the rank $n$ case of this. In other words, we suppose that $D \subset \widetilde{T}^{*} Y$ is a curve such that $\pi: D \rightarrow Y$ is generically an $n$-fold cover. $D$ moreover is endowed with a line bundle $\mathcal{L}$. Can we associate a $G L_{n}$ Higgs bundle to the pair $(D, \mathcal{L})$ ? We can define the bundle $E$ as usual by $E=\pi_{*}(\mathcal{L})$, and we can also imitate the usual definition of the Higgs field, $\varphi=\pi^{*}(z)$. Now, however, $\varphi$ is not a holomorphic map $E \rightarrow E \otimes K_{Y}$. Rather, each branch of $\varphi$, or more exactly the contribution to $\varphi$ from each branch of $\pi: D \rightarrow Y$, is twisted exactly as in Equation (4.21). Consequently, the equation obeyed by $\varphi$ is

$$
\bar{\partial}_{E} \varphi=1 \otimes \zeta
$$

where $\bar{\partial}_{E}$ is the $\bar{\partial}$ operator of the bundle $E$, and $1: E \rightarrow E$ is the identity.

This type of twisting makes sense for Hitchin's Equations (2.1), which we can slightly generalize to

$$
\begin{aligned}
F-\phi \wedge \phi & =1 \otimes i \zeta^{\prime} \\
d_{A} \star \phi & =1 \otimes i \operatorname{Im} \zeta \\
d_{A} \phi & =1 \otimes i \operatorname{Re} \zeta .
\end{aligned}
$$

Along with the deformation by $\zeta$ seen in the last paragraph, which has been rewritten in terms of real differential geometry, we have included an additional real ${ }^{25}$ closed $(1,1)$-form $\zeta^{\prime}$ in the first equation. Up to trivial equivalence, the equations depend only on the cohomology classes of $\zeta^{\prime}, \operatorname{Im} \zeta$,

\footnotetext{
${ }^{24}$ Of course, on a curve, every $(1,1)$-form is closed, but the construction is actually meaningful in higher dimensions, as we discuss in Section 4.9.

${ }^{25}$ The reason for the factors of $i$ on the right-hand sides of these equations is that $\phi$ and $F$ are forms valued in the Lie algebra of $U_{n}$, the compact form of $G L_{n}$; we represent this Lie algebra by anti-Hermitian $n \times n$ matrices.
} 
$\operatorname{Re} \zeta$. Those classes are a triple of real parameters, like the usual parameters $(\alpha, \beta, \gamma)$ of ramified Higgs bundles.

We expect that $A$-branes in $\widetilde{T}^{*} Y$ should be mapped to twisted $\mathcal{D}$ modules on $Y$ by solving this twisted form of Hitchin's equations. The right choice of $\zeta^{\prime}$ is not determined by the geometry of the $A$-brane, somewhat like the value of $\alpha^{*}$ in Section 4.4. This issue will be discussed in Section 4.7 .

After solving the twisted equations (4.23), we form the complex-valued connection $\mathcal{A}=A+i \phi$. Usually, Hitchin's equations imply that $\mathcal{A}$ is flat. In the present case, however, $\mathcal{A}$ is not flat, but rather has central curvature $\mathcal{F}_{0}=i\left(\zeta^{\prime}+i \operatorname{Re} \zeta\right)$. A connection with central curvature $\mathcal{F}_{0}$ describes not a $\mathcal{D}$-module but a twisted $\mathcal{D}$-module, twisted by $\mathcal{T}_{\chi}$, a "line bundle" of first Chern class $\chi=\mathcal{F}_{0} / 2 \pi i$. In a more intrinsic description, the twisting is really by $K_{Y}^{1 / 2} \otimes \mathcal{T}_{\chi}$, where the role of $K_{Y}^{1 / 2}$ has been described earlier.

\subsection{The $B$-field}

At two points we have run into important parameters that are not determined by the geometry of an $A$-brane, namely the ramification parameter $\alpha^{*}$ at a pole of $D \subset \widetilde{T}^{*} Y$, and the twisting class $\zeta^{\prime}$ of Equation (4.23). In the abstract, any values of these parameters make sense and can be used to construct twisted $\mathcal{D}$-modules by solving the twisted version of Hitchin's equations with singularities. We would like to know what values are relevant in the context of geometric Langlands.

For the twisting parameter, we can make an argument based on holomorphy that is very similar to what we have already said for the ramification parameter. The cohomology class $\chi$, like the twisting parameter $\varrho^{*}$, must be holomorphic in ${ }^{\mathrm{L}} \alpha-i^{\mathrm{L}} \gamma$. As we have just seen, $\operatorname{Im} \chi$ is determined by the affine deformation by which a Zariski open set in $\mathcal{M}_{\mathrm{H}}$ differs from the cotangent bundle $T^{*} \mathcal{M}$. In [30], by analyzing the geometry of $\mathcal{M}_{\mathrm{H}}$, it was shown that this is linear in ${ }^{\mathrm{L}} \gamma$, and hence $\operatorname{Re} \chi$ is similarly linear in ${ }^{\mathrm{L}} \alpha$.

However, we would like to explain more directly how $\zeta^{\prime}=\operatorname{Re} \chi$ originates in the quantum field theory. Up until this point, in our study of $A$-branes on a symplectic manifold such as $X=\widetilde{T}^{*} Y$, we have omitted an important aspect of the $A$-model, namely the $B$-field. Thus, we have defined the $A$-model purely in terms of the symplectic form $\omega$ of $X$. In general, the $A$-model depends holomorphically on the complexified symplectic form $\widehat{\omega}=\omega+i B$.

In the absence of the $B$-field, $A$-branes on a Lagrangian submanifold $X$ are characterized by a rank $n$ vector bundle $V \rightarrow L$ with a flat unitary 
connection $A$. (For brevity, we omit here the twisting by $K_{L}^{1 / 2}$.) Thus, writing $F$ for the curvature of $A$, the condition is $F=0$. For $B \neq 0$, however, the condition is modified to include a central curvature: ${ }^{26}$

$$
F=1 \otimes i B
$$

Thus, the twisting field $\zeta^{\prime}$ of Equation (4.23) is precisely $B . B$ is really only defined modulo the addition of an exact two-form.

As analyzed in [30], under duality from the $B$-model of ${ }^{\mathrm{L}} G$ to the $A$-model of $G,{ }^{\mathrm{L}} \alpha$ maps to a quantum parameter $\eta$ of the $A$-model, and in turn the $B$-field on $\mathcal{M}_{\mathrm{H}}(G)$ is linear in $\eta$, in the following sense. $\eta$ takes values in $\mathfrak{t}$, the Lie algebra of the compact form of $G$. The cohomology class $[B]$ of $B$ is an element of $H^{2}\left(\mathcal{M}_{\mathrm{H}}, \mathbb{R}\right)$, which has a natural identification as $\mathbb{R} \oplus \mathbf{t}$. (For simplicity, we consider the case of only one ramification point; otherwise, one has a similar analysis with more parameters.) Relative to this, $[B]=c \oplus \eta$, where $c$ is a constant that vanishes in the usual case of geometric Langlands (and is nonzero in a generalization known as quantum geometric Langlands).

Consider first the case that $\mathcal{M}_{\mathrm{H}}^{0}$ reduces to $T^{*} \mathcal{M}$. This actually occurs if ${ }^{\mathrm{L}} \beta={ }^{\mathrm{L}} \gamma=0$, as shown in [30]. Then $Y=\mathcal{M}$ can be embedded as a Lagrangian submanifold. Consider the special case of Equation (4.23) with $\varphi=0$. If we set $\zeta=0$ and $\zeta^{\prime}=B$, this reduces to Equation (4.24) and therefore is the right equation to describe a rank $n A$-brane supported on $\mathcal{M} \subset T^{*} \mathcal{M}$. Therefore the twisting parameter $\zeta^{\prime}$ of the category of $A$-branes is precisely $B$, or more precisely, its cohomology class; hence, $\zeta^{\prime}$ coincides with the ramification parameter ${ }^{\mathrm{L}} \alpha$ of the underlying ${ }^{\mathrm{L}} G$ ramified local system. Solutions with $\varphi \neq 0$ describe the possible deformation of the $A$ brane from a rank $n$ brane supported on $\mathcal{M}$ to a rank 1 brane supported on a more general Lagrangian submanifold $D \subset T^{*} \mathcal{M}(D$ is an $n$-fold cover of $\mathcal{M}$ ). A-branes supported on $D$ are twisted in the same way, since the twisting applies to the whole $A$-category.

In geometric Langlands, we are actually interested in a case in which $D \subset T^{*} \mathcal{M}$ has poles. However, $B$ when restricted to $T^{*} \mathcal{M}$ is a pullback from $\mathcal{M}$ (modulo addition of an irrelevant exact two-form) and the twisting is just as in the last paragraph. Turning on ${ }^{\mathrm{L}} \beta$ and ${ }^{\mathrm{L}} \gamma$, which are multiples of $\beta$ and $\gamma$, deforms the classical geometry from $T^{*} \mathcal{M}$ to $\widetilde{T}^{*} \mathcal{M}$, but (as

\footnotetext{
${ }^{26}$ Physicists usually omit the factor of $i$ on the right-hand side because of defining $F$ to take values in hermitian rather than anti-Hermitian $n \times n$ matrices.
} 
analyzed in [30]) does not change the cohomology class of $B$ and so does not affect this contribution to the twisting.

In summary, in the standard case of geometric Langlands, with one ramification point, $\zeta^{\prime}$ is equal to $0 \oplus^{\mathrm{L}} \alpha$. With $k$ ramification points, $H^{2}\left(\mathcal{M}_{\mathrm{H}}, \mathbb{R}\right)$ $=\mathbb{R} \oplus\left(\oplus_{i=1}^{k} \mathfrak{t}^{(i)}\right)$, where the $\mathfrak{t}^{(i)}$ are copies of $\mathfrak{t}$. Each ramification point has a corresponding parameter $\mathrm{L}^{(i)}$ and a similar argument shows that $\zeta^{\prime}=0 \oplus\left(\oplus_{i=1}^{k} \mathrm{~L}_{\alpha}^{(i)}\right)$.

4.7.1. Conjectural description of $\boldsymbol{\alpha}_{i}^{*}$ Now that we have determined $\zeta^{\prime}$, we discuss the parameters $\alpha_{i}^{*}$, where $i$, as before, labels the divisors in $\mathcal{M}$ where the Hitchin fiber "goes to infinity."

Here some general remarks on $B$-fields might be helpful. A general $B$-field of the type that we consider describes mathematically a $U(1)$-gerbe with a connection (similar objects will also be discussed in Section 10). A gauge transformation of such an object is made by taking the tensor product with a line bundle $\mathcal{L}$ with a unitary connection $\nabla$. In our situation, the gerbe is actually trivial, though not canonically so, which is why its connection may be represented by the globally defined two-form $B$ that was discussed above. Under a gauge transformation, this two-form gets shifted by the curvature of $\nabla$.

Now let $\boldsymbol{F}$ be a compact fiber of the Hitchin fibration, and $\boldsymbol{F}_{0}$ its restriction to $\widetilde{T}^{*} \mathcal{M}$. The cohomology class of $B$ restricted to $\boldsymbol{F}$ actually vanishes. This can be seen from the analysis in [30] and ensures that a rank 1 $A$-brane supported on $\boldsymbol{F}$ can exist. Such an $A$-brane is defined by giving a flat trivialization $\mathcal{T}$ of the gerbe, that is, a trivialization relative to which the connection form vanishes. (Often, it is assumed that the gerbe has an a priori trivialization, and then the $A$-brane is defined by a flat line bundle over $\boldsymbol{F}$. However, the intrinsic formulation is that the $A$-brane is defined by a flat trivialization of the gerbe.) On the other hand, upon restricting $B$ to $\boldsymbol{F}_{0}$, we may use a gauge in which $B$ is a pullback from $\mathcal{M}$. As such, $B$ may well be nonzero, but we can take it to be smooth and hence bounded in norm. The difference between the two gauges is given by a line bundle $\mathcal{L} \rightarrow \boldsymbol{F}_{0}$ with a connection $\nabla$. The connection is not flat, but its curvature is uniformly bounded, since $B$ is bounded on $\mathcal{M}$. Therefore, the monodromies of $\mathcal{L}$ around the divisors in $\boldsymbol{F} \backslash \boldsymbol{F}_{0}$ are well defined. We conjecture that these are actually responsible for the ramification parameters $\alpha_{i}^{*}$.

More precisely, in the neighborhood of each divisor $D_{i}$ in $\mathcal{M}$ over which the Hitchin fiber $\boldsymbol{F}$ goes to infinity, $\boldsymbol{F}$ has $n$ branches. Therefore, the connection $\nabla$ gives rise to a collection of monodromies $\lambda_{i}^{j} \in U(1), j=1, \ldots, n$, one for each branch. We conjecture that $\exp \left(-2 \pi \alpha_{i}^{*}\right)$ is in the conjugacy 
class of $\operatorname{diag}\left(\lambda_{i}^{1}, \ldots, \lambda_{i}^{n}\right)$. This completes our (conjectural) description of the $\alpha_{i}^{*}$.

Finally, we obtain the following description of the $\mathcal{D}$-module $\mathcal{F}$ associated to the $A$-brane $(\boldsymbol{F}, \mathcal{T})$. The curvature of $\mathcal{L} \rightarrow \boldsymbol{F}_{0}$ is of type $(1,1)$, since $B$ has this property, so the connection $\nabla$ endows $\mathcal{L}$ with a holomorphic structure. We extend $\mathcal{L}$ as a holomorphic line bundle over $\boldsymbol{F}$ by saying that a holomorphic section $\psi$ of $\mathcal{L} \rightarrow \boldsymbol{F}_{0}$ is holomorphic on $\boldsymbol{F}$ if its magnitude $|\psi|$, computed using the unitary structure of $\mathcal{L}$, is bounded. Moreover, we say that $\psi$ vanishes to order $\alpha$ near an irreducible component $D^{\prime}$ of $\boldsymbol{F} \backslash \boldsymbol{F}_{0}$ if near $D^{\prime}$ one has $|\psi| \sim|z|^{\alpha}$, with $z$ a normal coordinate near $D^{\prime}$. This characterization of the order of vanishing of a section is well defined because the curvature of $\mathcal{L}$ is bounded. It endows $\mathcal{L} \rightarrow \boldsymbol{F}$ with parabolic structure along $\boldsymbol{F} \backslash \boldsymbol{F}_{0}$.

Then $E=\pi_{*}(\mathcal{L})$, where $\pi: \boldsymbol{F}_{0} \rightarrow \mathcal{M}$ is the projection, has a natural parabolic structure at the divisors $D_{i}$. It is also equipped in the usual way with a natural Higgs field $\varphi$.

Our proposal is that the restriction of $\mathcal{F}$ to the complement of the divisors $D_{i}$ is a local system obtained by the non-abelian Hodge transformation of the parabolic Higgs bundle $(E, \varphi)$.

\subsection{Tame and irregular singularities}

So far we have considered only the case that the exponents $d_{i}$ describing the singular behavior of the Lagrangian curve $D \subset T^{*} Y$

$$
s_{i}(u) \sim c_{i}\left(u-u_{0}\right)^{-d_{i}}, \quad i=1, \ldots, n
$$

are equal to 1 . Here we will briefly relax this assumption.

One case is that the exponents are positive (so $D$ does go to infinity) but less than 1 . This is only possible if $D$, upon being completed by adding a point or points at infinity, is ramified over $u_{0}$. For example, taking $u_{0}=0$, let us consider a spectral curve $\operatorname{det}(s-\varphi)=0$ with $\operatorname{det} \varphi=1 / u$. The equation for the spectral curve is then $s^{2}+u^{-1}=0$, with branches $s= \pm(-u)^{1 / 2}$, so the exponents $d_{i}$ are equal to $1 / 2$. A suitable choice of $\varphi$ is

$$
\varphi=d u\left(\begin{array}{rc}
0 & u^{-1} \\
-1 & 0
\end{array}\right)
$$

$\varphi$ has a pole, but the polar residue is nilpotent, so det $\varphi$ has only a single pole, not a double one. The analysis of [68] applies to this situation; it corresponds to $\beta^{*}=\gamma^{*}=0$, as is reviewed in [30, Section 3.3]. Since $\gamma^{*}=0$, 
monodromies of the local system on $Y$ arising from an $A$-brane supported on $D$ will have modulus 1 .

An opposite case is that the exponents $d_{i}$ are greater than 1 . In this case, $\varphi$ has a pole at $u_{0}$ of greater than first order. Hitchin's equations for Higgs bundles in which $\varphi$ has such an irregular pole have been analyzed in [11]. The corresponding local system has an irregular singularity at $u_{0}$. The irregular part of the connection can be read off directly from the singularity of the Higgs field, in contrast to the subtle interplay of parameters $\alpha, \beta$ and $\gamma$ that arises in the regular case. For an informal explanation, see [79].

\subsection{The multi-dimensional case}

For geometric Langlands, we require a generalization of all this to $A$-branes in $\widetilde{T}^{*} Y$, where $Y$ has dimension greater than 1 . The correspondence between Higgs bundles and local systems has an extension to higher dimensions [69, $70]$, and this also generalizes to Higgs bundles with poles [10,59]. The generalization to allow poles is crucial for geometric Langlands, because a fiber $\boldsymbol{F}$ of the Hitchin fibration, when restricted to $T^{*} \mathcal{M}$, essentially always has poles. However, here we will consider only the case without poles, which will suffice for explaining the basic idea.

A Higgs bundle on a complex manifold $Y$ of dimension $n$ is defined to be a pair $(E, \varphi)$, where $E \rightarrow Y$ is a holomorphic vector bundle, and $\varphi: E \rightarrow E \otimes \Omega^{1}(Y)$ is a holomorphic map such that $\varphi^{2}=0\left(\varphi^{2}\right.$ is defined by composing endomorphisms of $E$ and using the cup product $\left.\wedge^{2} \Omega^{1} \rightarrow \Omega^{2}\right)$. The last condition is trivial in the case of a curve. The basic result is that if $Y$ is Kahler, then there is a natural correspondence between Higgs bundles on $Y$ such that the rational Chern classes of $E$ vanish and local systems on $Y$. The correspondence is made by solving a multi-dimensional generalization of Hitchin's equation. (If $Y$ is projective, this operation is equivalent to solving Hitchin's equations simultaneously on every curve in $Y$.)

There is also a natural correspondence between a Higgs bundle $(E, \varphi)$ and a spectral variety $D \subset T^{*} Y$ endowed with a line bundle $\mathcal{L}$. Letting $\pi: T^{*} Y \rightarrow Y$ denote the projection, the correspondence is made in one direction by setting $E=\pi_{*}(\mathcal{L})$ and $\varphi=\pi_{*}(z)$, where $z$ is linear on the fiber of the cotangent bundle.

We want to state this correspondence for a rank $1 A$-brane supported on a smoothly embedded compact Lagrangian submanifold $D \subset T^{*} Y$. (Examples of this situation for $\operatorname{dim} Y>1$ are actually extremely scarce, and this is another reason that the extension to allow poles of $D$ is essential.) Such 
a rank $1 A$-brane exists if and only if the canonical bundle of $D$ admits a square root (this is related to Remark 4.1). To specify such a brane, we pick a flat $\operatorname{Spin}_{c}$ structure over $D$ that we describe somewhat nonintrinsically by a choice of line bundle $K_{D}^{1 / 2} \otimes \mathcal{N}$, where $\mathcal{N}$ has zero first Chern class. Then we define ${ }^{27}$ a vector bundle $E \rightarrow Y$ by $K_{Y}^{1 / 2} \otimes E=\pi_{*}\left(K_{D}^{1 / 2} \otimes \mathcal{N}\right)$, or in other words $E=\pi_{*}(\mathcal{L})$ with $\mathcal{L}=K_{D}^{1 / 2} \otimes \pi^{*}\left(K_{Y}^{-1 / 2}\right) \otimes \mathcal{N}$. This extends to a Higgs bundle $(E, \varphi)$ with $\varphi=\pi_{*}(z)$. The rank of $E$ coincides with the degree $n$ of the cover $\pi: D \rightarrow Y$.

The Higgs bundle $(E, \varphi)$ will correspond to a local system over $Y$ if the Chern character of $E$, which we denote as $\operatorname{ch}(E)$, is equal to $n$. An argument via Riemann-Roch shows that this is always the case. In stating this argument, we use the $\widehat{A}$ class, which is related to the Todd class of a complex manifold $X$ by $\widehat{A}(X)=\operatorname{Td}(X) \operatorname{ch}\left(K_{X}^{1 / 2}\right)$. The $\widehat{A}$ class is defined for any real vector bundle $V$ and has the property that $\widehat{A}(V)=\widehat{A}\left(V^{*}\right)$, where $V^{*}$ is the dual to $V$.

The Riemann-Roch formula says in this situation that

$$
\pi_{*}\left(\operatorname{Td}(D) \operatorname{ch}\left(K_{D}^{1 / 2}\right)\right)=\operatorname{Td}(Y) \operatorname{ch}\left(K_{Y}^{1 / 2}\right) \operatorname{ch}(E)
$$

We have used the fact that as $c_{1}(\mathcal{N})=0, \operatorname{ch}(\mathcal{N})=1$. In terms of $\widehat{A}$ classes, this says that

$$
\pi_{*}(\widehat{A}(D))=\widehat{A}(Y) \operatorname{ch}(E) .
$$

Hence $\operatorname{ch}(E)$ equals $n$ if and only if $\pi_{*}(\widehat{A}(D))=n \widehat{A}(Y)$. Since this is a statement in rational cohomology, it is equivalent to show that

$$
2 \pi_{*}(\widehat{A}(D))=2 n \widehat{A}(Y) .
$$

Now by definition, $\widehat{A}(D)$ is the $\widehat{A}$ class of the tangent bundle $T D$ of $D$. So $2 \widehat{A}(D)=\widehat{A}(T D \oplus T D)$. As $D \subset T^{*} Y$ is Lagrangian, the normal bundle and tangent bundle to $D$ are isomorphic (as real vector bundles). Their direct sum is $\left.T\left(T^{*} Y\right)\right|_{D}$, that is, the restriction to $D$ of the tangent bundle

\footnotetext{
${ }^{27}$ If $K_{Y}$ does not have a global square root, then $E$ must be understood as a twisted vector bundle, twisted by a certain gerbe. In any event, that is the most intrinsic formulation.
} 
$T\left(T^{*} Y\right)$ to $T^{*} Y$. So we can replace $T D \oplus T D$ by $\left.T\left(T^{*} Y\right)\right|_{D}$, and interpret $2 \pi_{*}(\widehat{A}(D))$ as $\pi_{*}\left(\widehat{A}\left(\left.T\left(T^{*} Y\right)\right|_{D}\right)\right)$. As $Y \subset T^{*} Y$ is also Lagrangian, we can similarly interpret $2 \widehat{A}(Y)$ as $\widehat{A}\left(\left.T\left(T^{*} Y\right)\right|_{Y}\right)$. So the formula we want is that

$$
\pi_{*}\left(\widehat{A}\left(\left.T\left(T^{*} Y\right)\right|_{D}\right)\right)=n \widehat{A}\left(\left.T\left(T^{*} Y\right)\right|_{Y}\right)
$$

Since $T^{*} Y$ is contractible to $Y$, any vector bundle $V \rightarrow T^{*} Y$ is isomorphic to the pullback from $Y$ of its restriction to $Y$, that is, $V \cong \pi^{*}\left(\left.V\right|_{Y}\right)$. Setting $V=T\left(T^{*} Y\right)$, we have $T\left(T^{*} Y\right)=\pi^{*}\left(\left.T\left(T^{*} Y\right)\right|_{Y}\right)$. Hence in particular $\left.T\left(T^{*} Y\right)\right|_{D}$ is the restriction to $D$ of $\pi^{*}\left(\left.T\left(T^{*} Y\right)\right|_{Y}\right)$. The left-hand side of Equation (4.30) is therefore $\pi_{*}\left(\widehat{A}\left(\pi^{*}\left(\left.T\left(T^{*} Y\right)\right|_{Y}\right)\right)\right)$. In general, for any map $\pi: X \rightarrow Y$ and real vector bundle $V \rightarrow Y$, one has $\widehat{A}\left(\pi^{*}(V)\right)=\pi^{*}(\widehat{A}(V))$. So the left-hand side of $(4.30)$ is $\pi_{*}\left(\pi^{*}\left(\widehat{A}\left(\left.T\left(T^{*} Y\right)\right|_{Y}\right)\right)\right)$. For a map $\pi: D \rightarrow Y$ of degree $n$, the composition $\pi_{*} \pi^{*}$ acting on cohomology is multiplication by $n$, and now the validity of Equation (4.30) is clear.

The local system on $Y$ corresponding to the Higgs bundle $(E, \varphi)$ is then the desired $\mathcal{D}$-module corresponding to our starting point, the rank $1 A$ brane supported on the Lagrangian submanifold $D \subset T^{*} Y$. This is a very special case since we have assumed $D$ to be compact. In most of the examples relevant to the geometric Langlands program (such as the ones considered earlier in this section), this is not so, and the corresponding $\mathcal{D}$-modules are not represented by local systems on the entire $Y$, only on an open subset of $Y$. We have explained in Sections 4.5 to 4.7 how to construct the corresponding local systems on an open subset of $Y$ in the case when $\operatorname{dim} Y=1$. A similar picture hopefully holds in the multi-dimensional case. For some of the necessary analysis, see $[10,59]$.

\section{Spectral covers, Hecke operators and higher genus}

The analysis of Section 3 was primarily based on direct computations. One can learn much more using the technique of spectral curves, which was briefly introduced in Section 4.1. In Section 5.1, we reconsider the genus 1 example from this point of view; in Section 5.2, we apply similar ideas to the case of genus greater than 1 , and in Section 5.3, we use these methods to analyze the action of Hecke operators on $A$-branes arising in the geometric Langlands program. 


\subsection{Genus 1 revisited}

We return first to the example of Section 3, involving a curve $C$ of genus 1 , described by a cubic equation

$$
y^{2}=f(x)
$$

where $f(x)=\left(x-e_{1}\right)\left(x-e_{2}\right)\left(x-e_{3}\right)$. We consider $S L_{2} \operatorname{Higgs}$ bundles $(E, \varphi)$ ramified at the point $p$ defined by $x=y=\infty$. Ramification means that near $p$, the eigenvalues of $\varphi$ behave as $\pm \sigma_{0} d x / 2 x$, and hence $\operatorname{det} \varphi \sim-\sigma_{0}^{2} d x^{2} /$ $4 x^{2} \sim-\left(\sigma_{0}^{2} / 4\right) x(d x / y)^{2}$. Suppose that $\sigma_{0} \neq 0$. Then the general form of a quadratic differential on $C$ that is holomorphic except for this behavior near $p$ is $\operatorname{det} \varphi=-\left(\sigma_{0}^{2} / 4\right)(x-a)(d x / y)^{2}$, for some complex constant $a$. So after absorbing in $z$ a factor of $(d x / y) \sigma_{0} / 2$, Equation (4.1) of the spectral curve $D$ becomes

$$
z^{2}=x-a
$$

$D$ is, therefore, a cover of the $x$-plane described by the pair of Equations (5.1) and (5.2). Eliminating $x$ by means of the second equation, the spectral curve can be described by the equation

$$
y^{2}=\prod_{i=1}^{3}\left(z^{2}+a-e_{i}\right) .
$$

$D$ is a smooth curve of genus 2 , unless $a$ is equal to $e_{1}, e_{2}$ or $e_{3}$, in which case $D$ reduces to a curve of genus 1 with two points identified. For example, if $a=e_{1}$, we let $w=y / z$, obeying

$$
w^{2}=\left(z^{2}+e_{1}-e_{2}\right)\left(z^{2}+e_{1}-e_{3}\right) .
$$

This equation describes a smooth curve $D^{\prime}$ of genus 1. $D$ is obtained from $D^{\prime}$ by identifying the two points with $z=0, w= \pm \sqrt{\left(e_{1}-e_{2}\right)\left(e_{1}-e_{3}\right)}$. These two points, which we will call $q^{\prime}$ and $q^{\prime \prime}$, correspond to just one point $q$ in $D$, as they are both characterized by $y=z=0$.

$D^{\prime}$ is an unramified double cover of $C$. Indeed, $D^{\prime}$ has the freely acting symmetry $\tau: w \rightarrow-w, z \rightarrow-z$. The invariants are $x=z^{2}+e_{1}$, as well as $w^{2}$, which can be expressed in terms of $x$ via Equation (5.4), and $y=z w$, which obeys $y^{2}=\prod_{i=1}^{3}\left(x-e_{i}\right)$, the defining equation of $C$. So $C$ is the quotient $D^{\prime} /\{1, \tau\}$. 
We write $p^{\prime}$ and $p^{\prime \prime}$ for the two points in $D^{\prime}$ with $z=\infty$; they lie above the point $p$ at infinity in $C$. The symmetry $\tau$ of $D^{\prime}$ acts freely and is of order 2 , so if we regard $D^{\prime}$ as an elliptic curve with, say, $p^{\prime}$ as the origin, then $\tau$ is the shift by an element of order 2 . This element is simply $p^{\prime \prime}$, since $\tau$ exchanges $p^{\prime}$ and $p^{\prime \prime}$. If $r$ is any point in $C$ and $r^{\prime}, r^{\prime \prime}$ are the points in $D^{\prime}$ lying above $r$, then $\tau$ exchanges $r^{\prime}, r^{\prime \prime}$ so

$$
r^{\prime \prime}-r^{\prime}=p^{\prime \prime}-p^{\prime}
$$

The divisor $p^{\prime}+p^{\prime \prime}-q^{\prime}-q^{\prime \prime}$ is the divisor of the function $z$, so its divisor class is trivial. Together with Equation (5.5), this implies that the points $q^{\prime}, q^{\prime \prime}$ are of order 2 .

5.1.1. Fiber of the Hitchin fibration If $D$ is smooth, the fiber of the Hitchin fibration is a smooth curve of genus 1, the Prym variety of the double cover $\pi: D \rightarrow C$. Let us discuss what happens when $D$ is singular, for example at $a=e_{1}$.

We want to find the line bundles, or more generally torsion-free sheaves, on $D$ that push down to vector bundles $E \rightarrow C$ of trivial determinant. We will describe our line bundles and sheaves in terms of data on the smooth curve $D^{\prime}$. So let us begin by "pushing down" the trivial line bundle $\mathcal{O}_{D^{\prime}}$, using the projection $\rho: D^{\prime} \rightarrow C$. We compute the pushdown using the fact that the quotient $D^{\prime} /\{1, \tau\}$ is $C$. We can decompose $\rho_{*}\left(\mathcal{O}_{D^{\prime}}\right)$ in subsheaves that are even or odd under $\tau$. The even part is $\mathcal{O}_{C}$, and the odd part is a locally free sheaf $\mathcal{S} \rightarrow C$. $\mathcal{S}$ is uniquely determined by the fact that it is nontrivial and $\rho^{*}\left(\mathcal{S}^{-1}\right)$ has a global section over $D^{\prime}$. We can describe $\mathcal{S}$ via the divisor $-p+q$, since this pulls back on $D^{\prime}$ to the trivial divisor $-p^{\prime}-p^{\prime \prime}+q^{\prime}+q^{\prime \prime}$

Thus, $\mathcal{O}_{D^{\prime}}$ does not have the property that $\rho_{*}\left(\mathcal{O}_{D^{\prime}}\right)$ has trivial determinant. Its determinant is $\mathcal{O}(p)^{-1} \otimes \mathcal{O}(q)$. However, let $p^{*}$ be either of the points $p^{\prime}, p^{\prime \prime}$ and let $q^{*}$ be either of the points $q^{\prime}, q^{\prime \prime}$. Let $\mathcal{T}_{0}=\mathcal{O}\left(p^{*}\right) \otimes$ $\mathcal{O}\left(q^{*}\right)^{-1}$. Then $\operatorname{det}\left(\rho_{*}\left(\mathcal{T}_{0}\right)\right)=\mathcal{O}_{C}$. All we need here is that $p^{*}$ pushes down to $p$ and $q^{*}$ to $q$; this implies that $\operatorname{det}\left(\rho_{*}\left(\mathcal{T}_{0}\right)\right)=\mathcal{O}(p) \otimes \mathcal{O}(q)^{-1} \otimes$ $\operatorname{det}\left(\rho_{*}\left(\mathcal{O}_{D^{\prime}}\right)\right)=\mathcal{O}_{C}$. It looks like we have 4 choices of $\mathcal{T}$, but up to isomorphism there are only 2; in view of Equation (5.5), if we reverse the choice of $q^{*}$ while also reversing the choice of $p^{*}, \mathcal{T}$ is unchanged.

So we have found two line bundles $\mathcal{T}_{0} \rightarrow D^{\prime}$ that push down to $S L_{2^{-}}$ bundles $E \rightarrow C$. They are the only ones. But if we work on the singular curve $D$ rather than its normalization $D^{\prime}$, there are more choices. We first replace the degree zero line bundle $\mathcal{T}_{0}$ with the degree 1 line bundle $\mathcal{T}_{1}\left(p^{*}\right)=$ $\mathcal{O}\left(p^{*}\right)$. We think of $\mathcal{T}_{1}\left(p^{*}\right)$ as a line bundle on $D^{\prime}$ that is trivialized away 
from $p^{*}$. We cannot interpret $\mathcal{T}_{1}\left(p^{*}\right)$ as a line bundle on $D$ (as opposed to $\left.D^{\prime}\right)$ since in its definition, we have not taken account of the identification of the two points $q^{\prime}$ and $q^{\prime \prime}$ on $D^{\prime}$ to a single point $q \in D$. To do this, we pick $\lambda \in \mathbb{C}^{\times}$and define a line bundle $\mathcal{T}\left(\lambda ; p^{*}\right)$ over $D$ by saying that a section of $\mathcal{T}\left(\lambda ; p^{*}\right)$ is a section $f$ of $\mathcal{T}_{1}$ such that $f\left(q^{\prime}\right)=\lambda f\left(q^{\prime \prime}\right)$. This gives (since there are two choices of $\left.p^{*}\right)$ two families of complex line bundles over $D$, each parametrized by $\mathbb{C}^{\times}$.

To compactify these families, we set $\lambda=-v / u$, where $u$ and $v$ will be understood as homogeneous coordinates for $\mathbb{C P}^{1}$, and replace the condition $f\left(q^{\prime}\right)=\lambda f\left(q^{\prime \prime}\right)$ by

$$
u f\left(q^{\prime}\right)+v f\left(q^{\prime \prime}\right)=0 .
$$

For any $u, v$, the sheaf of sections of $\mathcal{T}_{1}\left(p^{*}\right)$ that obey this condition is a torsion-free sheaf $\mathcal{R}\left(u, v ; p^{*}\right)$ on $D$ whose pushdown to $C$ is a rank two vector bundle of trivial determinant. If $u, v \neq 0$, this sheaf is locally free and is the sheaf of sections of the line bundle $\mathcal{T}\left(-v / u ; p^{*}\right)$. If $u$ or $v$ vanishes, we get a torsion-free but not locally free sheaf on $D$. This torsion-free sheaf is the pullback from $D^{\prime}$ of the line bundle $\mathcal{O}\left(p^{*}\right) \otimes \mathcal{O}\left(q^{*}\right)^{-1}$, where $q^{*}$ is $q^{\prime}$ if $v=0$ (and Equation (5.6) reduces to $f\left(q^{\prime}\right)=0$ ), or $q^{\prime \prime}$ if $u=0$.

The four line bundles $\mathcal{O}\left(p^{*}\right) \otimes \mathcal{O}\left(q^{*}\right)^{-1} \rightarrow D^{\prime}$, with the different choices of $p^{*}$ and $q^{*}$, are isomorphic in pairs, because of the relation (5.5), with $r=q$. Consequently, $\mathcal{R}\left(0,1 ; p^{\prime}\right)$ is isomorphic to $\mathcal{R}\left(1,0 ; p^{\prime \prime}\right)$, and similarly with $p^{\prime}, p^{\prime \prime}$ exchanged.

This construction gives two families $\mathcal{R}\left(u, v ; p^{*}\right)$ of torsion-free sheaves on $D$. Each family is parametrized by $\mathbb{C P}^{1}$, with homogeneous coordinates $u, v$. The two $\mathbb{C P}^{1}$ 's meet at two points, because of the remark in the last paragraph. The fiber of the Hitchin fibration for $S L_{2}$ is the union of these $\mathbb{C P}^{1}$ 's. This agrees with the picture that we developed in Section 3.4 by direct computation.

5.1.2. The improper component We can similarly use spectral curves to describe the Hitchin fibration for the improper component of the $S L_{2}$ moduli space, introduced in Section 3.8. This component parametrizes Higgs bundles $(E, \varphi)$ with $\operatorname{det} E=\mathcal{O}(r), r$ being a point in $C$. Here we will treat an issue that was omitted in Section 3.8: the dependence on $r$. So we refer to the improper component as $\mathcal{M}_{\mathrm{H}}\left(S L_{2}^{*} ; r\right)$.

This space is independent of $r$ up to a not quite canonical isomorphism; given another point $\widetilde{r}$, we pick a line bundle $\mathcal{N}$ whose square is isomorphic to $\mathcal{O}(\widetilde{r}) \otimes \mathcal{O}(r)^{-1}$, and then tensoring with $\mathcal{N}$ gives a map from $\mathcal{M}_{\mathrm{H}}\left(S L_{2}^{*} ; r\right)$ to $\mathcal{M}_{\mathrm{H}}\left(S L_{2}^{*} ; \widetilde{r}\right)$. The choice of $\mathcal{N}$ is unique modulo tensoring with a line 
bundle of order 2 , so the identification of $\mathcal{M}_{\mathrm{H}}\left(S L_{2}^{*} ; r\right)$ with $\mathcal{M}_{\mathrm{H}}\left(S L_{2}^{*} ; \widetilde{r}\right)$ is unique modulo the action of the group $Q$ of line bundles of order 2. (Hence $\mathcal{M}_{\mathrm{H}}\left(S L_{2}^{*} ; r\right)$ becomes naturally independent of $r$ if one divides by $Q$; this gives the moduli space $\mathcal{M}_{\mathrm{H}}\left(\mathrm{SO}_{3} ; \theta\right)$ of $\mathrm{SO}_{3}$ Higgs bundles with nonzero second Stieffel-Whitney class, whose definition requires no choice of $r$.) If $\widetilde{r}$ is close to $r$, we can resolve the ambiguity by asking that $\mathcal{N}$ should be near the identity (in the Picard group of $C$ ), so locally there is a natural identification of $\mathcal{M}_{\mathrm{H}}\left(S L_{2}^{*} ; r\right)$ with $\mathcal{M}_{\mathrm{H}}\left(S L_{2}^{*} ; \widetilde{r}\right)$. Hence there is a natural monodromy action, the group of monodromies being simply $Q$.

Regardless of $\operatorname{det} E$, the spectral curve for a Higgs bundle $(E, \varphi)$ is defined by the equation $\operatorname{det}(z-\varphi)=0$. Hence, the relevant spectral curves $D$ for the improper component of the Hitchin fibration are the same as for the proper component. The difference is only that now the fiber of the Hitchin fibration parametrizes line bundles $\mathcal{R} \rightarrow D$, or more generally torsion-free sheaves, such that $\operatorname{det}\left(\pi_{*}(\mathcal{R})\right)=\mathcal{O}(r)\left(\right.$ rather than $\left.\operatorname{det}\left(\pi_{*}(\mathcal{R})\right)=\mathcal{O}\right)$. Just as before, the fiber of the Hitchin fibration is smooth if $D$ is smooth; we want to consider the special fibers for which $D$ is not smooth, but has for normalization a smooth genus 1 curve $D^{\prime}$.

To construct those special fibers, we repeat the previous construction, now beginning not with the line bundle $\mathcal{O}\left(p^{*}\right) \rightarrow D^{\prime}$, but with $\mathcal{T}_{1}=\mathcal{O}\left(p^{*}\right) \otimes$ $\mathcal{O}\left(r^{*}\right)$, where $r^{*}$ is either of $r^{\prime}, r^{\prime \prime}$. There are seemingly four choices of $\mathcal{T}_{1}$, involving the choices of $p^{*}$ and $r^{*}$, but since $r^{\prime \prime}-r^{\prime}=p^{\prime \prime}-p^{\prime}$ on the elliptic curve $D^{\prime}$, there are only two choices up to isomorphism. Just as for the proper component of the moduli space, we associate to either of these line bundles over $D^{\prime}$ a family $\mathcal{T}\left(u, v ; p^{*}, r^{*}\right)$ of torsion-free sheaves on $D$, by imposing Equation (5.6). Each family is parametrized by $\mathbb{C P}^{1}$, and, as before, $\mathcal{T}\left(0,1 ; p^{*}, r^{\prime}\right)$ is isomorphic to $\mathcal{T}\left(1,0 ; p^{*}, r^{\prime \prime}\right)$, and vice versa. So the two $\mathbb{C P}^{1}$ 's meet at two points. Their union is the fiber of the Hitchin fibration.

Now we can consider monodromies when $r$ varies in $C$. These will exchange the two choices of $r^{*}$, and so will exchange the two components of the Hitchin fiber. This agrees with the fact that the monodromy group is the group $Q$ of line bundles of order 2, and that the action of $Q$ exchanges the two components of the Hitchin fiber, as we saw in Section 3.5.

\subsection{Extension to higher genus}

Our next goal is to apply the same methods to the case that $C$ is a smooth curve of genus $g>1$. We will see that the results are similar. For simplicity, 
we omit ramification. For $g=1$, the unramified case is rather degenerate, but that is not so for $g>1$.

5.2.1. The spectral curves What sort of Higgs bundles over $C$ will be related to endoscopy? We consider endoscopic $\mathrm{SO}_{3}$ local systems whose structure group reduces to $\mathrm{O}_{2}$, the subgroup consisting of elements of the form

$$
\left(\begin{array}{rrr}
* & * & 0 \\
* & * & 0 \\
0 & 0 & \pm 1
\end{array}\right)
$$

but not to a proper subgroup. For such local systems, the automorphism group is equal to $\mathbb{Z}_{2}$. The correspondence between local systems and Higgs bundles given by Hitchin's equations is compatible with any reduction of the structure group. So $\mathrm{SO}_{3}$ local systems with structure group reducing to $\mathrm{O}_{2}$ correspond to Higgs bundles with the same structure group. Those which do not further reduce to a proper subgroup have the group of automorphisms equal to $\mathbb{Z}_{2}$. From now on, we will restrict ourselves to these Higgs bundles.

If we lift such a Higgs bundle to $S L_{2}$, the structure group lifts to what we will call $\mathrm{O}_{2}^{*}$, a double cover of $\mathrm{O}_{2}$ generated by the diagonal elements of $S L_{2}$

$$
\left(\begin{array}{ll}
* & 0 \\
0 & *
\end{array}\right)
$$

together with the element

$$
\left(\begin{array}{rr}
0 & 1 \\
-1 & 0
\end{array}\right)
$$

An $S L_{2}$ spectral curve $D$ is defined by an equation

$$
z^{2}+\operatorname{det} \varphi=0
$$

where $\operatorname{det} \varphi$ is a quadratic differential on $C$. For $C$ of genus $g$, a quadratic differential has $4 g-4$ zeros. $D$ is smooth if and only if the zeros of $\operatorname{det} \varphi$ are distinct, in which case the genus of $D$ is $4 g-3$.

What happens when the structure group reduces to $O_{2}^{*}$ ? The Lie algebra of $O_{2}^{*}$ is simply the abelian algebra of traceless diagonal matrices. So if $(E, \varphi)$ is an $S L_{2}$ Higgs bundle whose structure group reduces to $O_{2}^{*}$, then $\varphi$ locally 
takes the form

$$
\varphi=\left(\begin{array}{cc}
\omega & 0 \\
0 & -\omega
\end{array}\right),
$$

where $\omega$ is a holomorphic differential on $C$. This leads to $\operatorname{det} \varphi=-\omega^{2}$, as a result of which any zeros of $\operatorname{det} \varphi$ are double zeros. Near a double zero at, say, $x=0$, with $x$ a local parameter on $C$, the equation for $D$ looks something like $z^{2}-x^{2}=0$; the point $z=x=0$ is a double point. Each double point reduces by 1 the genus of the normalization of $D$. For an $\mathrm{O}_{2}^{*}$ Higgs bundle, the zeros are all double zeros, so there are $2 g-2$ double points and the normalization of $D$ is a curve $D^{\prime}$ of genus $2 g-1$.

The fact that the zeros of $\operatorname{det} \varphi$ are double zeros does not imply that globally $\operatorname{det} \varphi=-\omega^{2}$ for a holomorphic section $\omega$ of the canonical bundle $K$. Rather, it implies that $\operatorname{det} \varphi=-\omega^{2}$, where $\omega$ is a holomorphic section of $K \otimes \mathcal{V}$, for some line bundle $\mathcal{V} \rightarrow C$ of order 2 . The case of interest to us is that $\mathcal{V}$ is nontrivial. (The case that $\mathcal{V}$ is trivial is related to Higgs bundles whose structure group reduces to $G L_{1}$ rather than $O_{2}^{*}$.) Associated with the choice of $\mathcal{V}$ is an unramified double cover $\pi^{\prime}: D^{\prime} \rightarrow C$. This is a curve of genus $2 g-1$ and is the normalization of $D$. Let $\tau: D^{\prime} \rightarrow D^{\prime}$ be the covering map that commutes with $\pi^{\prime}$. Then $C=D^{\prime} /\{1, \tau\}$. The element $\omega \in H^{0}(C, K \otimes \mathcal{V})$ vanishes at $2 g-2$ points $p_{1}, \ldots, p_{2 g-2}$. Above each such point $p_{i}$, there are two points $p_{i}^{\prime}, p_{i}^{\prime \prime}$ in $D^{\prime}$, exchanged by $\tau$, but only a single point $\widehat{p}_{i} \in D$. $D$ is obtained from $D^{\prime}$ by gluing together the pairs of points $p_{i}^{\prime}$ and $p_{i}^{\prime \prime}$.

What we have just described is a natural analog of the result of Section 5.1 for a genus 1 curve $C$ with one point of ramification. A curve of genus 1 has precisely three nontrivial unramified double covers; these are the normalizations $D^{\prime}$ of the three singular spectral curves $D$.

5.2.2. The Prym An open dense subset of the Hitchin fiber $\boldsymbol{F}$ for $S L_{2}$ consists of line bundles $\mathcal{L} \rightarrow D$ such that $\operatorname{det} \pi_{*}(\mathcal{L})=\mathcal{O}$. The full Hitchin fiber parametrizes certain torsion-free sheaves as well as these line bundles.

Given such a line bundle $\mathcal{L}$, let $\mathcal{L}^{\prime}$ be its pullback to $D^{\prime}$. Then $\operatorname{det} \pi_{*}^{\prime}\left(\mathcal{L}^{\prime}\right)$

$=\mathcal{O}\left(p_{1}+\cdots+p_{2 g-2}\right)$. (When $\mathcal{L}$ is lifted to $D^{\prime}$, we drop the requirement that a section must have equal values at the points $p_{i}^{\prime}$ and $p_{i}^{\prime \prime}$ lying above $p_{i}$, and this leads to the claimed result.) The space of such line bundles is ${ }^{28}$ a

\footnotetext{
${ }^{28}$ This statement means that if $\mathcal{L}^{\prime}$ and $\mathcal{L}^{\prime \prime}$ are two line bundles with $\operatorname{det} \pi_{*}^{\prime}\left(\mathcal{L}^{\prime}\right)=\operatorname{det} \pi_{*}^{\prime}\left(\mathcal{L}^{\prime \prime}\right)=\mathcal{O}\left(p_{1}+\cdots+p_{2 g-2}\right)$, then $\mathcal{L}^{\prime \prime}=\mathcal{L}^{\prime} \otimes \mathcal{N}$ for a unique $\mathcal{N}$ with $\operatorname{det} \pi_{*}^{\prime}(\mathcal{N})=\mathcal{O}$.
} 
"torsor" for the group of line bundles $\mathcal{N} \rightarrow D^{\prime}$ with $\operatorname{det} \pi_{*}^{\prime}(\mathcal{N})=\mathcal{O}$. Since $\pi^{\prime}: D^{\prime} \rightarrow C$ is unramified, $\operatorname{det} \pi_{*}^{\prime}(\mathcal{N})$ is the same as $\operatorname{Nm}(\mathcal{N})$, the 29 "norm" of $\mathcal{N}$. The group of line bundles of trivial norm is called the Prym variety of $\pi^{\prime}: D^{\prime} \rightarrow C$. (Sometimes the term "Prym variety" is taken to refer to the connected component of this group.) We write $\boldsymbol{P}$ for the Prym and $\boldsymbol{T}$ for its torsor of line bundles $\mathcal{L}^{\prime} \rightarrow D^{\prime}$ obeying $\operatorname{det} \pi_{*}^{\prime}\left(\mathcal{L}^{\prime}\right)=\mathcal{O}\left(p_{1}+\cdots+p_{2 g-2}\right)$.

We can easily construct a large family of line bundles over $D^{\prime}$ of trivial norm. We take any points $s_{i} \in C, i=1, \ldots, k$ (allowing some of the points to coincide), denote as $s_{i}^{\prime}$ and $s_{i}^{\prime \prime}$ the points in $D^{\prime}$ lying above $s_{i}$ (with any choice of which one is which), and define a line bundle $\mathcal{N}$ by

$$
\mathcal{N}=\bigotimes_{i=1}^{k} \mathcal{O}\left(s_{i}^{\prime}-s_{i}^{\prime \prime}\right)
$$

Conversely, one can show that every point of $\boldsymbol{P}$ can be represented by a line bundle of this form. To see that (we thank T. Pantev for showing us this argument), let $E$ be a divisor on $D^{\prime}$ such that $\mathcal{N}=\mathcal{O}(E)$. Then $\operatorname{Nm}(\mathcal{N})=\mathcal{O}_{C}\left(\pi^{\prime}(E)\right)$. If $\mathcal{N} \in \boldsymbol{P}$, then $\pi^{\prime}(E)=(f)$, the divisor of a rational function $f$ on $C$. But it follows from Tsen's theorem that the norm map from the field of rational functions on $D^{\prime}$ to the field of rational functions on $C$ is surjective. ${ }^{30}$ Hence there exists a rational function $g$ on $D^{\prime}$ whose norm is $f$. Then $\pi^{\prime}((g))=(f)$. Let $E^{\prime}=D-(g)$. Then $\pi^{\prime}\left(E^{\prime}\right)=0$ and therefore $E^{\prime}$ has the form $\sum_{i=1}^{k}\left(s_{i}^{\prime}-s_{i}^{\prime \prime}\right)$. Thus, we obtain Equation (5.12).

Now let us find the connected components of the Prym. Obviously, the part of $\boldsymbol{P}$ that we can construct for fixed $k$ is connected, since $C$ itself is connected and the points $s_{i}$ may move freely. Line bundles that differ by an exchange $s_{i}^{\prime} \leftrightarrow s_{i}^{\prime \prime}$ lie in the same connected component of $\boldsymbol{P}$, since these two points are exchanged under monodromy of $s_{i}$. Line bundles that differ by changing $k$ by a multiple of 2 are also in the same connected component; $k$ is reduced by 2 if we take 2 of the $s_{i}$ to be equal, with the points labeled $s_{i}^{\prime}$ and $s_{i}^{\prime \prime}$ chosen properly, and use the identity $\left(s_{i}^{\prime}-s_{i}^{\prime \prime}\right)+\left(s_{i}^{\prime \prime}-s_{i}^{\prime}\right)=0$.

On the other hand, $\boldsymbol{P}$ actually has two connected components. This is shown in [60], and also follows by a purely topological argument (see the discussion of Equation (5.15)). ${ }^{31}$ The argument in the last paragraph shows

\footnotetext{
${ }^{29}$ For any map of curves $\pi: D \rightarrow C$, the norm is a map from line bundles over $D$ to line bundles over $C$ defined as follows. The norm of $\mathcal{N}=\mathcal{O}\left(\sum_{i} n_{i} p_{i}\right) \rightarrow D$, for integers $n_{i}$ and points $p_{i} \in D$, is defined as $\operatorname{Nm}(\mathcal{N})=\mathcal{O}\left(\sum_{i} n_{i} \pi\left(p_{i}\right)\right)$.

${ }^{30}$ The norm of a rational function $g$ on $D^{\prime}$ is by definition the product of $g$ and $\tau(g)$, where $\tau$ is the involution on $D^{\prime}$ corresponding to the cover $D^{\prime} \rightarrow C$.

${ }^{31}$ Another proof is presented in [64, Section 11].
} 
that the only possible invariant is the value of $k$ modulo 2 . So it must be that the component of $\boldsymbol{P}$ connected to the identity is characterized by even $k$, while the disconnected component is characterized by odd $k$.

The torsor $\boldsymbol{T}$ is noncanonically isomorphic to $\boldsymbol{P}$, so it likewise has two components. We proceed as in Section 5.1.1 to construct the fiber $\boldsymbol{F}$ of the Hitchin fibration from $\boldsymbol{T}$. Let $\mathcal{L}^{\prime}$ be any line bundle representing a point in $\boldsymbol{T}$. For $i=1, \ldots, 2 g-2$, pick a pair of homogeneous coordinates $\left(u_{i}, v_{i}\right)$, and define a line bundle $\mathcal{L}^{\prime}\left(u_{1}, v_{1} ; \ldots ; u_{2 g-2}, v_{2 g-2}\right) \rightarrow D$ by saying that a section of this line bundle is a section $f$ of $\mathcal{L}^{\prime} \rightarrow D^{\prime}$ that obeys

$$
u_{i} f\left(p_{i}^{\prime}\right)+v_{i} f\left(p_{i}^{\prime \prime}\right)=0, \quad i=1, \ldots, 2 g-2 .
$$

If, for all $i, u_{i}$ and $v_{i}$ are both nonzero, this construction gives a family of line bundles over $D$, representing (if we also let $\mathcal{L}^{\prime}$ vary in $\boldsymbol{T}$ ) a Zariski open set of the Hitchin fiber $\boldsymbol{F}$. This open set has two connected components $\widetilde{\boldsymbol{F}}_{1}$ and $\widetilde{\boldsymbol{F}}_{2}$, because $\mathcal{L}^{\prime}$ may lie in either component of $\boldsymbol{T}$. They are isomorphic to $\left(\mathbb{C}^{\times}\right)^{2 g-2}$-bundles over the two components of $\boldsymbol{T}$.

As in Section 5.1.1, to get the full Hitchin fiber, we must compactify by including torsion-free sheaves that are obtained by allowing $u_{i}$ or $v_{i}$ to vanish, for each $i$. The compactified fiber has two irreducible components, which we call $\boldsymbol{F}_{1}$ and $\boldsymbol{F}_{2}$. They are isomorphic to $\left(\mathbb{C P}^{1}\right)^{2 g-2}$-bundles over the two components of $\boldsymbol{T}$. However, just as in the genus 1 example, $\boldsymbol{F}_{1}$ and $\boldsymbol{F}_{2}$ intersect over the divisors on which $u_{i}$ or $v_{i}$ vanish. The reason for this is that starting with a line bundle $\mathcal{L}^{\prime} \rightarrow D^{\prime}$ and taking $u_{i}=0$ in the construction of the last paragraph is equivalent to starting with a different line bundle $\mathcal{L}^{\prime \prime}=$ $\mathcal{L}^{\prime} \otimes \mathcal{O}\left(p_{i}^{\prime}-p_{i}^{\prime \prime}\right)$ and taking $v_{i}=0$. But the operation $\mathcal{L}^{\prime} \rightarrow \mathcal{L}^{\prime \prime}$ exchanges the two connected components of $\boldsymbol{T}$.

5.2.3. The improper component We also want to understand the Higgs bundles $(E, \varphi)$ where $\operatorname{det} E=\mathcal{O}(r), r$ being a specified point in $C$. The Hitchin fiber in this case can be analyzed via the same methods. We simply have to start with a different torsor $\boldsymbol{T}(r)$ for the same Prym variety $\boldsymbol{P}$. $\boldsymbol{T}(r)$ parametrizes line bundles $\mathcal{L}^{\prime} \rightarrow D^{\prime}$ with $\operatorname{det} \pi_{*}^{\prime}\left(\mathcal{L}^{\prime}\right)=\mathcal{O}\left(p_{1}+\right.$ $\left.\cdots+p_{2 g-2}+r\right) . \boldsymbol{T}(r)$ again has two connected components, exchanged by tensor product with $\mathcal{O}\left(s^{\prime}-s^{\prime \prime}\right)$ for any $s \in C$. Correspondingly, the fiber of the Hitchin fibration has two components, meeting along the divisors that parametrize torsion-free sheaves that are not locally free.

Let $\boldsymbol{F}$ and $\boldsymbol{F}^{*}(r)$ be the Hitchin fibers for Higgs bundles $(E, \varphi)$ with, respectively, $\operatorname{det} E=\mathcal{O}$ and $\operatorname{det} E=\mathcal{O}(r)$. Then $\boldsymbol{F}$ and $\boldsymbol{F}^{*}(r)$ are noncanonically isomorphic. To make an isomorphism, we simply pick a point 
$r^{\prime} \in D^{\prime}$ lying above $r$. Then, for $\mathcal{L}^{\prime} \in \boldsymbol{T}$, we define a line bundle $\mathcal{L}_{r^{\prime}}^{\prime} \in \boldsymbol{T}(r)$ by $\mathcal{L}_{r^{\prime}}^{\prime}=\mathcal{L}^{\prime} \otimes \mathcal{O}\left(r^{\prime}\right)$. We map $\boldsymbol{F}$ to $\boldsymbol{F}^{*}(r)$ by $\mathcal{L}^{\prime}\left(u_{i}, v_{i}\right) \rightarrow \mathcal{L}_{r^{\prime}}^{\prime}\left(u_{i}, v_{i}\right)$. This is an isomorphism between $\boldsymbol{F}$ and $\boldsymbol{F}^{*}(r)$, but it is not quite canonical since it depends on the choice of $r^{\prime}$.

Now consider the effect of a monodromy in $r$ that exchanges the two points $r^{\prime}$ and $r^{\prime \prime}$. The effect of this is to map $\mathcal{L}_{r^{\prime}}^{\prime}$ to $\mathcal{L}_{r^{\prime \prime}}^{\prime}=\mathcal{L}_{r^{\prime}}^{\prime} \otimes \mathcal{O}\left(r^{\prime \prime}-r^{\prime}\right)$. This operation exchanges the two components of $\boldsymbol{F}^{*}(r)$. Thus, monodromy in $r$ exchanges the two components of $\boldsymbol{F}^{*}(r)$, just as we saw in the genus 1 example at the end of Section 5.1.2.

5.2.4. Topological point of view Here we will explain from a topological point of view the fact that the Prym $\boldsymbol{P}$ for an unramified (but connected) double cover $D^{\prime} \rightarrow C$ has two components.

This Prym is the fiber of the Hitchin fibration for certain $O_{2}^{*}$ local systems. We recall that $O_{2}^{*}$ is the subgroup of $S L_{2}$ generated by the diagonal matrices together with the element

$$
\left(\begin{array}{rr}
0 & 1 \\
-1 & 0
\end{array}\right) \text {. }
$$

Under the double cover $\mathrm{SL}_{2} \rightarrow \mathrm{SO}_{3}, \mathrm{O}_{2}^{*}$ projects to $\mathrm{O}_{2} \subset \mathrm{SO}_{3}$. Topologically, $\mathrm{O}_{2}^{*}$ has two components; the component containing the identity consists of diagonal elements, and the other component consists of elements of $\mathrm{O}_{2}^{*}$ that are not diagonal.

The statement that the Prym has two components is equivalent to the statement that even after we pick an unramified double cover $D^{\prime} \rightarrow C$, the corresponding component of $\mathcal{M}_{\mathrm{H}}\left(O_{2}^{*}\right)$ actually has two components. (The base of the Hitchin fibration for a given $D^{\prime}$ is connected, so the components of $\mathcal{M}_{\mathrm{H}}\left(O_{2}^{*}\right)$ are simply the components of the Prym.) Using the relation of $\mathrm{O}_{2}^{*}$ Higgs bundles to $\mathrm{O}_{2}^{*}$ local systems, the question of determining the components of $\mathcal{M}_{\mathrm{H}}\left(\mathrm{O}_{2}^{*}\right)$ is equivalent to the analogous question about $O_{2}^{*}$ local systems and can be answered topologically.

Picking suitable generators of the fundamental group of $C$, and writing $A_{i}, B_{j}, i, j=1, \ldots, g$ for the monodromies of an $O_{2}^{*}$ local system, we have

$$
\left[A_{1}, B_{1}\right]\left[A_{2}, B_{2}\right] \cdots\left[A_{g}, B_{g}\right]=1
$$

where $[A, B]=A B A^{-1} B^{-1}$. We specify $\mathcal{V}$ by saying, for example, that $B_{g}$ lies in the disconnected component of $O_{2}^{*}$ and all other $A_{i}$ and $B_{j}$ in the connected component. Since the connected component is abelian, Equation (5.15) reduces to $A_{g} B_{g} A_{g}^{-1} B_{g}^{-1}=1$, which implies (for $B_{g}$ in the 
disconnected component) that $A_{g}$ is one of the two central elements of $O_{2}^{*}$. The two components of $\boldsymbol{P}$ are distinguished by the choice of $A_{g}$.

5.2.5. Analog for $\mathrm{SO}_{3}$ To understand endoscopy, we must consider the fiber of the Hitchin fibration for $S O_{3}$ rather than $S L_{2}$. Just as in Equation (3.20), the moduli space of $\mathrm{SO}_{3}$ Higgs bundles is obtained from the moduli space of $S L_{2}$ Higgs bundles by dividing by the group $Q=H^{1}\left(C, \mathbb{Z}_{2}\right)$ of line bundles of order 2. $Q$ acts on a Higgs bundle $(E, \varphi)$ by $E \rightarrow E \otimes \mathcal{R}$, where $\mathcal{R}$ is a line bundle of order 2 .

This operation does not affect $\operatorname{det} \varphi$, so the action of $Q$ commutes with the Hitchin fibration. The action on a fiber of the Hitchin fibration is easily described; if $E=\pi_{*}(\mathcal{L})$ for a line bundle $\mathcal{L}$ over the spectral cover, then $E \otimes \mathcal{R}=\pi_{*}\left(\mathcal{L} \otimes \pi^{*}(\mathcal{R})\right)$. So the action of $Q$ on the fiber of the Hitchin fibration is by $\mathcal{L} \rightarrow \mathcal{L} \otimes \pi^{*}(\mathcal{R})$.

In the case of a smooth spectral curve $D$, the fiber $\boldsymbol{F}$ of the Hitchin fibration is a complex torus, and the operation $\mathcal{L} \rightarrow \mathcal{L} \otimes \pi^{*}(\mathcal{R})$ is a translation on this torus. It acts without fixed points. Now let us see what happens in the case related to endoscopy, when the normalization of $D$ is an unramified double cover $D^{\prime} \rightarrow C$. $\boldsymbol{F}$ is usefully described, ${ }^{32}$ as we have seen, in terms of the torsor $\boldsymbol{T}$ that parametrizes line bundles $\mathcal{L}^{\prime} \rightarrow D^{\prime}$ with $\operatorname{det} \pi_{*}\left(\mathcal{L}^{\prime}\right)=\mathcal{O}\left(p_{1}+\cdots+p_{2 g-2}\right) . \quad Q$ acts on this torsor by $\mathcal{L}^{\prime} \rightarrow \mathcal{L}^{\prime} \otimes \mathcal{R}$. $Q$ also acts on the gluing data of Equation (5.13), as we will discuss momentarily.

A first basic fact about this endoscopic case is that [60] the action of $Q$ exchanges the two components of the Prym $\boldsymbol{P}$, and hence of the torsor $\boldsymbol{T}$. (Of course, a subgroup of $Q$ of index 2 maps a given component to itself.) This fact has a simple topological explanation using Equation (5.15). $Q$ acts by independent sign changes on all $A_{i}$ and $B_{j}$; the two components of $\boldsymbol{P}$ are exchanged by any element of $Q$ that changes the sign of $A_{g}$.

So after dividing by $Q$, the Hitchin fiber for $\mathrm{SO}_{3}$ has only one component, in contrast to the situation for $S L_{2}$. This is familiar from Section 3.6.

Second, and again familiar from Section 3.6, the moduli space of $\mathrm{SO}_{3}$ Higgs bundles has singularities that arise because the action of $Q$ is not quite free. How can this occur? If $\mathcal{R} \rightarrow C$ is a line bundle of order 2 whose pullback to $D^{\prime}$ is nontrivial, then the operation $\mathcal{L} \rightarrow \mathcal{L} \otimes \mathcal{R}$ acts freely on $\boldsymbol{T}$ and hence on $\boldsymbol{F}$.

However, $\pi^{\prime}: D^{\prime} \rightarrow C$ is an unramified double cover associated with a line bundle $\mathcal{V} \rightarrow C$ of order 2 , and tautologically the pullback $\left(\pi^{\prime}\right)^{*}(\mathcal{V})$ of $\mathcal{V}$ to $D^{\prime}$ is trivial. Hence the element of $Q$ corresponding to $\mathcal{V}$ acts trivially

${ }^{32}$ The action of $Q$ on the improper fiber $\boldsymbol{F}^{*}(r)$ can be considered similarly. 
on $\boldsymbol{T}$. But it acts nontrivially on the gluing condition of Equation (5.13). Triviality of $\left(\pi^{\prime}\right)^{*}(\mathcal{V})$ means that this line bundle has an everywhere nonzero global section $w$. This section is odd, rather than even, under the covering map $\tau: D^{\prime} \rightarrow D^{\prime}$. (Otherwise $w$ would descend to a section of $\mathcal{V} \rightarrow C$, contradicting the fact that $\mathcal{V}$ is nontrivial.) The action of $\mathcal{V}$ modifies the gluing condition of Equation (5.13) to $u_{i} \widetilde{f}\left(p_{i}^{\prime}\right)+v_{i} \tilde{f}\left(p_{i}^{\prime \prime}\right)=0$, where $\tilde{f}=f w$. Since $w$ is odd under the covering map, which exchanges $p_{i}^{\prime}$ and $p_{i}^{\prime \prime}$ for all $i$, the effect of this is to transform the gluing data by

$$
\left(u_{i}, v_{i}\right) \longrightarrow\left(u_{i},-v_{i}\right), i=1, \ldots, 2 g-2 .
$$

Bearing in mind that the pair $u_{i}$ and $v_{i}$ are homogeneous coordinates for a copy of $\mathbb{C P}^{1}$, the condition for a fixed point of $\mathcal{V}$ is that $u_{i}$ or $v_{i}$ must vanish for all $i$. This is $2 g-2$ conditions.

We also adjusted $2 g-2$ parameters so that the spectral curve $D$ has for its normalization a double cover $D^{\prime} \rightarrow C$. As a check, and also a confirmation that fixed points of this kind only occur for the sort of spectral curves that we have assumed, we observe that the fact that the $Q$ action preserves the complex symplectic structure of the moduli space of Higgs bundles, together with the Lagrangian nature of the Hitchin fibers, implies that the number of parameters on the fiber of the Hitchin fibration that must be adjusted to get a fixed point equals the number of parameters that must be adjusted on the base.

So altogether, the $\mathbb{Z}_{2}$ fixed points that we have found occur in codimension $4 g-4$. The local structure is $\mathbb{C}^{4 g-4} / \mathbb{Z}_{2}$. These singularities are not $A_{1}$ singularities, since they arise in complex codimension greater than 2 . But the generalities of Section 2.2 still apply. There are two inequivalent $B$-branes $\mathcal{B}_{+}$and $\mathcal{B}_{-}$supported at a $\mathbb{Z}_{2}$ orbifold singularity of any codimension; our basic proposal is that mirror symmetry maps this fact to the fact that the corresponding Hitchin fiber for $S L_{2}$ has two components, $\boldsymbol{F}_{1}$ and $\boldsymbol{F}_{2}$. Thus, two $B$-branes $\mathcal{B}_{+}$and $\mathcal{B}_{-}$give rise to two $A$-branes $\mathcal{A}_{1}$ and $\mathcal{A}_{2}$ supported on these two components. Which one of them corresponds to $\mathcal{B}_{+}$ and which to $\mathcal{B}_{-}$is a subtle issue, which will be discussed in Section 10 (this is the reason for the choice of notation, $\mathcal{A}_{1}, \mathcal{A}_{2}$, and not $\mathcal{A}_{+}, \mathcal{A}_{-}$).

The locus of $\mathbb{Z}_{2}$ singularities has dimension $2 g-2$. There are $g-1$ parameters in picking a spectral curve $z^{2}+\operatorname{det} \varphi=0$, where $\operatorname{det} \varphi$ has only double zeros, and $g-1$ more parameters in picking an appropriate line bundle $\mathcal{L}^{\prime} \rightarrow D^{\prime}$.

5.2.6. The transfer Let us now consider this result from the point of view of $\mathrm{SO}_{3}$ local systems. An $\mathrm{SO}_{3}$ local system should represent a $\mathbb{Z}_{2}$ 
orbifold singularity of $\mathcal{M}_{\mathrm{H}}$ if its structure group reduces to $\mathrm{O}_{2}$, but not to a proper subgroup thereof. The moduli space of $\mathrm{O}_{2}$ Higgs bundles has dimension $2 g-2$, which agrees with the dimension of the above-described family of orbifold singularities.

More specifically, the Higgs bundles representing the singularities are precisely $\mathrm{O}_{2}$ Higgs bundles. $\mathcal{M}_{\mathrm{H}}\left(\mathrm{O}_{2}\right)$ is simply the quotient by $Q=H^{1}\left(C, \mathbb{Z}_{2}\right)$ of $\mathcal{M}_{\mathrm{H}}\left(\mathrm{O}_{2}^{*}\right)$. So its Hitchin fibration is easily understood: the base is the same as it is for $O_{2}^{*}$, and the fiber is the quotient of the Prym by $Q$. This is the same as the singular locus of $\mathcal{M}_{\mathrm{H}}\left(\mathrm{SO}_{3}\right)$ that we have just described.

An argument just like that surrounding Equation (5.15) shows that $\mathcal{M}_{\mathrm{H}}\left(\mathrm{O}_{2}\right)$ has two components, even after an unramified double cover $D^{\prime} \rightarrow C$ is specified. Indeed, from the point of view of an $\mathrm{O}_{2}$ local system $U$, the choice of unramified double cover specifies the Stieffel-Whitney class $w_{1}(U)$, and the two components for a given choice of $w_{1}(U)$ differ by the value of $w_{2}(U)$. The map $U \rightarrow U \oplus \operatorname{det} U$ from an $\mathrm{O}_{2}$ local system to an $\mathrm{SO}_{3}$ local system kills $w_{1}$ and leaves $w_{2}$ unchanged. So all components of $\mathcal{M}_{\mathrm{H}}\left(O_{2}\right)$ with a given value of $w_{2}$ appear as $\mathbb{Z}_{2}$ orbifold singularities in the component of $\mathcal{M}_{\mathrm{H}}\left(S_{3}\right)$ labeled by the same value of $w_{2}$.

The fact that $\mathcal{M}_{\mathrm{H}}\left(\mathrm{O}_{2}\right)$ appears as a locus of singularities in $\mathcal{M}_{\mathrm{H}}\left(\mathrm{SO}_{3}\right)$ is not special to the pair $\mathrm{O}_{2}$ and $\mathrm{SO}_{3}$. For any reductive Lie group ${ }^{\mathrm{L}} \mathrm{G}$ and reductive subgroup ${ }^{\mathrm{L}} H$, one has a natural embedding $\mathcal{M}_{\mathrm{H}}\left({ }^{\mathrm{L}} H\right) \subset \mathcal{M}_{\mathrm{H}}\left({ }^{\mathrm{L}} G\right)$. If the centralizer of ${ }^{\mathrm{L}} H$ in ${ }^{\mathrm{L}} G$ is nontrivial, then $\mathcal{M}_{\mathrm{H}}\left({ }^{\mathrm{L}} H\right)$ will be a locus of singularities. This embedding leads to a natural functor (direct image) from the category of $B$-branes on $\mathcal{M}_{\mathrm{H}}\left({ }^{\mathrm{L}} H\right)$ to the category of $B$-branes on $\mathcal{M}_{\mathrm{H}}\left({ }^{\mathrm{L}} G\right)$, and this will have to give rise to a functor from the category of $A$-branes on $\mathcal{M}_{\mathrm{H}}(H)$ to the category of $A$-branes on $\mathcal{M}_{\mathrm{H}}(G)$, as shown on the following diagram:

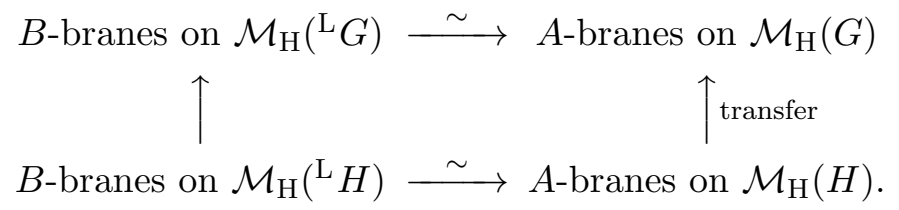

This is closely related to what in the Langlands program is called the transfer or the functoriality principle $[4,44]$. In the classical setting (discussed in more detail in Section 7), this means that for any homomorphism of the dual groups ${ }^{\mathrm{L}} H \rightarrow{ }^{\mathrm{L}} G$ one expects to have a map (the "transfer") from the set of equivalences classes of irreducible automorphic representations of $H(\mathbb{A})$ (more precisely, their $L$-packets) to those of $G(\mathbb{A})$. In the geometric setting, automorphic representations are replaced by $\mathcal{D}$-modules on 
$\operatorname{Bun}_{G}$, or $A$-branes on $\mathcal{M}_{\mathrm{H}}(G)$, and the transfer becomes a functor between appropriate categories associated to $H$ and $G$, as in the above diagram. This functor should be compatible with the action of the Hecke/'t Hooft operators (discussed in the next section) on the two categories.

Mirror symmetry of the Hitchin fibrations provides a natural setup for constructing such a functor. In physical terms, one might hope to study this situation by introducing a supersymmetric domain wall, with $\mathcal{N}=4$ supersymmetric Yang-Mills theory of gauge group ${ }^{\mathrm{L}} H_{\mathrm{c}}$ (the compact form of ${ }^{\mathrm{L}} H$ ) on one side of the domain wall, and the same theory with gauge group ${ }^{\mathrm{L}} G_{\mathrm{c}}$ on the other side. For some examples of string-theoretic constructions of such domain walls, see [78].

In our example ${ }^{\mathrm{L}} \mathrm{H}=\mathrm{O}_{2}$ and ${ }^{\mathrm{L}} \mathrm{G}=\mathrm{SO}_{3}$. On the dual side, we have $\mathrm{G}=$ $S L_{2}$ and $H(F)$ is a twisted torus in $S L_{2}(F)$, where $F$ is the field of rational functions on $C$, which is defined as follows. Consider the moduli space $\mathcal{M}_{\mathrm{H}}\left(\mathrm{O}_{2}, 0\right)$ of $\mathrm{O}_{2}$-Higgs bundles with $w_{2}=0$, embedded into $\mathcal{M}_{\mathrm{H}}\left(\mathrm{SO}_{3}, 0\right)$. It has components parametrized by the set $J_{2}$ of unramified double covers $D^{\prime} \rightarrow C$. For each $\psi \in J_{2}^{\times}$corresponding to an unramified cover, we define $H_{\psi}(F)$ as the group of nonzero rational functions $f$ on $D^{\prime}$ such that $\tau(f)=$ $f^{-1}$, where $\tau$ is the involution of the cover. It is naturally realized as a subgroup of $S L_{2}(F)$. The transfer functor of the diagram above is then constructed as follows.

Each component $\mathcal{M}_{\mathrm{H}}\left(\mathrm{O}_{2}, 0\right)_{\psi}$ of $\mathcal{M}_{\mathrm{H}}\left(\mathrm{O}_{2}, 0\right)$ corresponding to $\psi \in J_{2}^{\times}$is a toric fibration over the corresponding locus $\boldsymbol{B}_{\psi}$ in the Hitchin base $\boldsymbol{B}$. We have $\boldsymbol{B}_{\psi}=H^{0}\left(C, K \otimes \mathcal{L}_{\psi}\right)$, where $\mathcal{L}_{\psi}$ is the line bundle on $C$ corresponding to $\psi$, and the map $\boldsymbol{B}_{\psi} \rightarrow \boldsymbol{B}=H^{0}\left(C, K^{2}\right)$ is given by $\omega \mapsto \omega^{2}$. Since we wish to avoid the local systems that reduce to proper subgroups of $\mathrm{O}_{2}$, we consider the complement $\mathcal{M}_{\mathrm{H}}\left(\mathrm{O}_{2}, 0\right)_{\psi}^{\circ}$ of the zero fiber in $\mathcal{M}_{\mathrm{H}}\left(\mathrm{O}_{2}, 0\right)_{\psi}$. This is a subvariety in $\mathcal{M}_{\mathrm{H}}\left(S O_{3}, 0\right)$ projecting onto $\boldsymbol{B}_{\psi}^{\circ}=\left(\boldsymbol{B}_{\psi} \backslash 0\right) \subset \boldsymbol{B}$. The union of these varieties is precisely the "elliptic endoscopic locus" of $\mathcal{M}_{\mathrm{H}}\left(\mathrm{SO}_{3}, 0\right)$ (see [64] and Section 9). For each point $b \in \boldsymbol{B}_{\psi}^{\circ}$, the Hitchin fiber $\boldsymbol{F}_{b}\left(O_{2}\right)_{\psi} \subset$ $\mathcal{M}_{\mathrm{H}}\left(\mathrm{O}_{2}, 0\right)_{\psi}$ is identified with the moduli space of rank one unitary local systems on each of the two components, $\boldsymbol{F}_{b, 1}$ and $\boldsymbol{F}_{b, 2}$, of the corresponding singular Hitchin fiber $\boldsymbol{F}_{b}$ in the dual moduli space $\mathcal{M}_{\mathrm{H}}\left(S L_{2}\right)$. Indeed, as explained in Section 5.2.2, each $\boldsymbol{F}_{b, i}$ is isomorphic to a $\left(\mathbb{C P}^{1}\right)^{2 g-2}$-bundle over an abelian variety $\boldsymbol{F}_{b}\left(O_{2}\right)_{\psi}^{\vee}$, which is dual to $\boldsymbol{F}_{b}\left(O_{2}\right)_{\psi}$. The transfer (outside of the zero Hitchin fiber) is then implemented via the fiberwise $T$-duality of the toric fibration $\mathcal{M}_{\mathrm{H}}\left(O_{2}, 0\right)_{\psi}^{\circ}$. In particular, the skyscraper $B$-brane supported at a point $\mathcal{E} \in \boldsymbol{F}_{b}\left(O_{2}\right)_{\psi} \subset \mathcal{M}_{\mathrm{H}}\left(O_{2}, 0\right)_{\psi}^{\circ}$ gives rise to a magnetic eigenbrane on $\boldsymbol{F}_{b} \subset \mathcal{M}_{\mathrm{H}}\left(S L_{2}\right)$, which is the sum of the pullbacks of the corresponding rank one unitary local system on $\boldsymbol{F}_{b}\left(O_{2}\right)_{\psi}^{\vee}$ to $\boldsymbol{F}_{b, 1}$ and $\boldsymbol{F}_{b, 2}$. 
In addition, $\mathcal{M}_{\mathrm{H}}\left(\mathrm{O}_{2}\right)_{\psi}^{\circ}$ is embedded into $\mathcal{M}_{\mathrm{H}}\left(\mathrm{SO}_{3}\right)$ as the locus of $\mathbb{Z}_{2^{-}}$ orbifold singularities. This leads to a "doubling" of the category of $B$-branes supported on this component. On the dual side, this is reflected in the fact that the dual Hitchin fibers have two components, $\boldsymbol{F}_{b, 1}$ and $\boldsymbol{F}_{b, 2}$, also leading to a "doubling" of the corresponding category of $A$-branes.

More general examples will be considered in Section 9.

\section{3. 't Hooft/Hecke operators}

Much of the richness of the geometric Langlands program comes from the fact that the $\mathcal{D}$-modules dual to local systems are eigensheaves for the geometric Hecke operators. In the quantum field theory approach, this arises from a duality between line operators that are known as Wilson and 't Hooft operators.

We want to describe the refinement of this picture associated with endoscopy. For simplicity, we focus on our usual example with ${ }^{\mathrm{L}} \mathrm{G}=\mathrm{SO}_{3}$ and a local system with automorphism group $\mathbb{Z}_{2}$.

5.3.1. Review of Wilson operators First we describe the action of Wilson operators in general [30, Section 8]. We let $(\widehat{A}, \widehat{\phi})$ be the universal solution of the $\mathrm{SO}_{3}$ Hitchin equations over $\mathcal{M}_{\mathrm{H}}\left(\mathrm{SO}_{3}\right) \times C$. Thus, $\widehat{A}$ is a unitary connection on an $\mathrm{SO}_{3}$ bundle $\mathrm{W} \rightarrow \mathcal{M}_{\mathrm{H}}\left(S_{3}\right) \times C$, and $\widehat{\phi}$ a section of $\operatorname{ad}(\mathrm{W}) \otimes \Omega_{C}^{1} \rightarrow \mathcal{M}_{\mathrm{H}}\left(S O_{3}\right) \times C$, such that if we restrict to $m \times C$ for a point $m \in \mathcal{M}_{\mathrm{H}}\left(S O_{3}\right)$, then $(\widehat{A}, \widehat{\phi})$ is gauge-equivalent to the solution of Hitchin's equations determined by $m$. As usual, the restriction of $(\widehat{A}, \widehat{\phi})$ to $m \times C$ determines either an $\mathrm{SO}_{3}$ local system over $\mathrm{C}$ or an $\mathrm{SO}_{3}$ Higgs bundle.

A Wilson operator in ${ }^{\mathrm{L}} G$ gauge theory is associated to the choice of a point $p \in C$ and a representation ${ }^{\mathrm{L}} R$ of ${ }^{\mathrm{L}} G$. For simplicity, we will take ${ }^{\mathrm{L}} R$ to be the three-dimensional representation of ${ }^{\mathrm{L}} \mathrm{G}=\mathrm{SO}_{3}$, and we write $W_{p}$ for the corresponding Wilson operator. The action of $W_{p}$ on $B$-branes can be described as follows. Let $\mathcal{B}$ be a $B$-brane associated with a coherent sheaf (or a complex of coherent sheaves) $\mathcal{K} \rightarrow \mathcal{M}_{\mathrm{H}}$. Then $W_{p} \cdot \mathcal{B}$ is the $B$-brane associated with the sheaf $\left.\mathcal{K} \otimes W\right|_{p}$, where $\left.W\right|_{p}$ is the restriction of $W$ to $\mathcal{M}_{\mathrm{H}} \times p$. (We understand $W$ as a rank 3 vector bundle with structure group $\mathrm{SO}_{3}$.) Thus, the action of $W_{p}$ on coherent sheaves is

$$
\left.\mathcal{K} \rightarrow \mathcal{K} \otimes W\right|_{p}
$$

This formula makes sense for any of the complex structures that make up the hyper-Kahler structure of $\mathcal{M}_{\mathrm{H}}$, since $\mathrm{W}$, when restricted to $\mathcal{M}_{\mathrm{H}} \times p$, 
is holomorphic in any complex structure. (It carries a natural connection whose curvature is of type $(1,1)$ for each complex structure.) In geometric Langlands, one is interested primarily in complex structure $J$, in which $\mathcal{M}_{\mathrm{H}}\left(\mathrm{SO}_{3}\right)$ parametrizes $\mathrm{SO}_{3}$ local systems, but the existence of the other complex structures simplifies some computations, as we will see.

A very important special case is that the brane $\mathcal{B}$ is simultaneously a $B$-brane for each of the complex structures of $\mathcal{M}_{\mathrm{H}}$. We then call $\mathcal{B}$ a brane of type $(B, B, B)$ - a $\mathcal{B}$-brane in complex structures $I, J$ or $K$ (or any linear combination). Examples are a brane supported at a point in $\mathcal{M}_{\mathrm{H}}$ - the case that we consider momentarily - and a brane defined by an inclusion $\mathcal{M}_{\mathrm{H}}\left({ }^{\mathrm{L}} G^{\prime}\right) \subset \mathcal{M}_{\mathrm{H}}\left({ }^{\mathrm{L}} G\right)$, for some subgroup ${ }^{\mathrm{L}} G^{\prime} \subset{ }^{\mathrm{L}} G$. In this case, $W_{r} \cdot \mathcal{B}$ is again a brane of type $(B, B, B)$. Thus, the action of $W_{r}$ preserves the full topological symmetry of type $(B, B, B)$ (that is, of the $B$-model in any complex structure).

Of particular interest are the eigenbranes of the Wilson operators, also called electric eigenbranes. One defines the tensor product of a brane with a vector space $V$ as follows: if $\mathcal{B}$ is defined by a sheaf $\mathcal{K}$, then $\mathcal{B} \otimes V$ is defined by the sheaf $\mathcal{K} \otimes V$. A brane $\mathcal{B}$ is called an eigenbrane of $W_{p}$ if

$$
W_{p} \cdot \mathcal{B}=\mathcal{B} \otimes V_{p}
$$

for some vector space $V_{p}$. We will call $V_{p}$ the multiplier. In complex structure $J$, the $B$-model of $\mathcal{M}_{\mathrm{H}}$ is related to a four-dimensional topological field theory and general arguments can be used [30, Section 6.4] to show that if Equation (5.18) holds for one value of $p$, then it holds for all $p$ and $V_{p}$ varies as the fiber of a local system.

Comparing to (5.17), we see that for a brane $\mathcal{B}$ to be an electric eigenbrane, the bundle $\left.\mathrm{W}\right|_{p}$ must be trivial - equivalent to a constant vector space - when restricted to the support of $\mathcal{B}$. This is so if the support of $\mathcal{B}$ is a smooth point $m \in \mathcal{M}_{\mathrm{H}}\left(S_{3}\right)$. More specifically, we take $\mathcal{B}$ to be the brane (known in the physics literature as a zero-brane) associated with a skyscraper sheaf supported at $m$. Such a brane is an electric eigenbrane with multiplier the vector space $\left.W\right|_{m \times p}$, that is, the restriction of $W \rightarrow \mathcal{M}_{\mathrm{H}} \times C$ to the point $m \times p \in \mathcal{M}_{\mathrm{H}} \times C$ :

$$
W_{p} \cdot \mathcal{B}=\left.\mathcal{B} \otimes W\right|_{m \times p}
$$

This statement holds in any complex structure, so we can think of $\mathcal{B}$ as an eigenbrane of type $(B, B, B)$. In other words, it is a $B$-brane in complex structure $I, J$ or $K$ on $\mathcal{M}_{\mathrm{H}}$ (or any combination thereof), and furthermore is an eigenbrane in any complex structure. In the geometric 
Langlands program, one cares primarily about complex structure $J$, but the fact that the zero-brane is simultaneously an eigenbrane in all three complex structures facilitates computations, as will become clear.

5.3.2. $\boldsymbol{B}$-branes at an orbifold singularity We want to repeat this analysis for the case of a brane supported at a $\mathbb{Z}_{2}$ orbifold singularity $r \in \mathcal{M}_{\mathrm{H}}$. Such a singularity is associated with an $\mathrm{SO}_{3}$ local system whose structure group reduces to $\mathrm{O}_{2}$. We recall that $\mathrm{O}_{2}$ is embedded in $\mathrm{SO}_{3}$ as the subgroup

$$
\left(\begin{array}{rrr}
* & * & 0 \\
* & * & 0 \\
0 & 0 & \pm 1
\end{array}\right)
$$

and that any $\mathrm{SO}_{3}$ local system whose structure group reduces to $\mathrm{O}_{2}$ has symmetry group $\mathbb{Z}_{2}$, generated by the central element of $\mathrm{O}_{2}$ :

$$
\left(\begin{array}{rrr}
-1 & 0 & 0 \\
0 & -1 & 0 \\
0 & 0 & 1
\end{array}\right) \text {. }
$$

As usual, we will consider a generic local systems of this type whose group of automorphisms is precisely this $\mathbb{Z}_{2}$. In the present context, $\left.W\right|_{r}$, the restriction of $\mathrm{W}$ to $r \times C$, is a local system whose structure group reduces to $\mathrm{O}_{2}$, so it has a decomposition

$$
\left.\mathrm{W}\right|_{r}=U \oplus \operatorname{det} U,
$$

where $U$ is a rank 2 local system, with structure group $O_{2}$, and $\operatorname{det} U$ is its determinant. The central generator of $\mathbb{Z}_{2}$ acts as -1 on $U$ and as +1 on $\operatorname{det} U$.

As explained in Section 2.2, the category of branes supported at the orbifold singularity $r$ is generated by two irreducible objects $\mathcal{B}_{+}$and $\mathcal{B}_{-}$. Each is associated with a skyscraper sheaf supported at $r$. They differ by whether the nontrivial element of $\mathbb{Z}_{2}$ acts on this sheaf as multiplication by +1 or by -1 .

What happens when we act on $\mathcal{B}_{+}$or $\mathcal{B}_{-}$by the Wilson operator $W_{p}$ ? Since $\mathcal{B}_{+}$and $\mathcal{B}_{-}$both have skyscraper support at $r, W_{p}$ acts on either of them by tensor product with the three-dimensional vector space $\left.W\right|_{r \times p}$, the fiber of $W$ at $r \times p$. However, we should be more precise to keep track of the $\mathbb{Z}_{2}$ action. In view of Equation (5.22), there is a decomposition 
$\left.\mathrm{W}\right|_{r \times p}=\left.\left.U\right|_{p} \oplus \operatorname{det} U\right|_{p}$, where the nontrivial element of $\mathbb{Z}_{2}$ acts as -1 on the first summand and as +1 on the second summand. So we have

$$
\begin{aligned}
& W_{p} \cdot \mathcal{B}_{+}=\left(\left.\mathcal{B}_{-} \otimes U\right|_{p}\right) \oplus\left(\left.\mathcal{B}_{+} \otimes \operatorname{det} U\right|_{p}\right) \\
& W_{p} \cdot \mathcal{B}_{-}=\left(\left.\mathcal{B}_{+} \otimes U\right|_{p}\right) \oplus\left(\left.\mathcal{B}_{-} \otimes \operatorname{det} U\right|_{p}\right)
\end{aligned}
$$

A less precise but illuminating way to rewrite this is as follows. $\left.\operatorname{det} U\right|_{p}$ is a one-dimensional vector space on which $\mathbb{Z}_{2}$ acts trivially, so $\left.\mathcal{B}_{ \pm} \otimes \operatorname{det} U\right|_{p}$ is isomorphic, noncanonically, to $\mathcal{B}_{ \pm}$. And $\left.U\right|_{p}$ is a two-dimensional vector space on which the nontrivial element of $\mathbb{Z}_{2}$ acts as multiplication by -1 . So $\left.\mathcal{B}_{ \pm} \otimes U\right|_{p}$ is isomorphic, noncanonically, to the sum of two copies of $\mathcal{B}_{\mp}$. Thus up to isomorphism, we have

$$
\begin{aligned}
& W_{p} \cdot \mathcal{B}_{+}=\mathcal{B}_{+}+2 \mathcal{B}_{-} \\
& W_{p} \cdot \mathcal{B}_{-}=\mathcal{B}_{-}+2 \mathcal{B}_{+}
\end{aligned}
$$

We want to understand the magnetic dual of these statements. In Sections 5.3.3 and 5.3.4, we review the geometric Langlands duality for generic Hitchin fibers, and then in Section 5.3.5, we consider the behavior for special Hitchin fibers related to endoscopy.

5.3.3. $\varphi$-Invariant Hecke modifications The magnetic dual of a Wilson operator $W_{p}$ is an 't Hooft operator $T_{p}$. For the definition of 't Hooft operators and their relation to the usual Hecke operators of the geometric Langlands program, see Sections 9 and 10 of [39]. An $A$-brane $\mathcal{A}$ that is an eigenbrane for the 't Hooft operators, in the sense that, for every 't Hooft operator $T_{p}$,

$$
T_{p} \cdot \mathcal{A}=\mathcal{A} \otimes V_{p}
$$

for some vector space $V_{p}$, is known as a magnetic eigenbrane. Wilson operators of ${ }^{\mathrm{L}} G$ gauge theory are classified by a choice of representation of ${ }^{\mathrm{L}} G$, and 't Hooft operators of $G$ gauge theory are likewise classified by representations of ${ }^{\mathrm{L}} G$. Electric-magnetic duality is expected to map Wilson operators to 't Hooft operators and electric eigenbranes to magnetic eigenbranes.

Let us review the action of a 't Hooft operator $T_{p}$ on a Higgs bundle $(E, \varphi)$. In case $\varphi=0$, the possible Hecke modifications are the usual ones considered in the geometric Langlands program; they are parametrized by a subvariety of the affine Grassmannian known as a Schubert variety $\mathcal{V}$, which depends on a choice of representation ${ }^{\mathrm{L}} R$ of the dual group ${ }^{\mathrm{L}} G$. For instance, continuing with our example, if $G=S L_{2}$ and ${ }^{\mathrm{L}} R$ is the three-dimensional 
representation of ${ }^{\mathrm{L}} \mathrm{G}=\mathrm{SO}_{3}$, then a generic point in $\mathcal{V}$ corresponds to a Hecke modification of an $S L_{2}$ bundle $E$ of the following sort: for some local decomposition of $E$ as a sum of line bundles $\mathcal{N}_{1} \oplus \mathcal{N}_{2}, E$ is mapped to $\mathcal{N}_{1}(p) \oplus \mathcal{N}_{2}(-p)$. Letting $\mathcal{N}_{1}$ and $\mathcal{N}_{2}$ vary, this gives a two-parameter family of Hecke modifications of $E$. A family of modifications of $E$ of this type can degenerate to a trivial modification, and $\mathcal{V}$ contains a point corresponding to the trivial Hecke transformation.

What we have just described, for this example, is the possible action of the 't Hooft operator in the most degenerate case that $\varphi=0$. If instead $\varphi \neq 0$, one must restrict to Hecke modifications that are in a certain sense $\varphi$-invariant. For $G=S L_{2}$, and assuming $\varphi$ to be regular semi-simple at the point $p, \varphi$-invariance means that the decomposition $E=\mathcal{N}_{1} \oplus \mathcal{N}_{2}$ must be compatible with the action of $\varphi$, in the sense that $\varphi: E \rightarrow E \otimes K$ maps $\mathcal{N}_{1}$ to $\mathcal{N}_{1} \otimes K$ and $\mathcal{N}_{2}$ to $\mathcal{N}_{2} \otimes K$. There are precisely two possible choices of $\mathcal{N}_{1}$ and $\mathcal{N}_{2}$ : locally, as $\varphi(p)$ is regular semi-simple, we can diagonalize $\varphi$

$$
\varphi=\left(\begin{array}{rr}
a & 0 \\
0 & -a
\end{array}\right),
$$

and $\mathcal{N}_{1}$ and $\mathcal{N}_{2}$ must equal, up to permutation, the two "eigenspaces."

In addition to these two nontrivial $\varphi$-invariant Hecke modifications, we must remember to include the trivial Hecke modification (since it corresponds to a point in $\mathcal{V}$ ), which is also $\varphi$-invariant. Altogether then there are in this example three $\varphi$-invariant Hecke modifications, namely a trivial one and two nontrivial ones, a statement that, as we will see, is dual to the fact that the representation of ${ }^{\mathrm{L}} \mathrm{G}=\mathrm{SO}_{3}$ that we started with is three-dimensional.

Now let us see what the $\varphi$-invariant Hecke modifications look like from the point of view of the spectral curve $\pi: D \rightarrow C$. We consider first the case of a generic spectral curve, given by an equation $\operatorname{det}(z-\varphi)=0$. A $\varphi$-invariant Hecke modification leaves fixed the characteristic polynomial of $\varphi$ and hence maps each fiber $\boldsymbol{F}$ of the Hitchin fibration to itself. How does it act on $\boldsymbol{F}$ ?

A point $p \in C$ at which $\varphi$ is regular semi-simple lies under two distinct points $p^{\prime}, p^{\prime \prime} \in D$. The bundle $E$ is $\pi_{*}(\mathcal{L})$ for some line bundle $\mathcal{L} \rightarrow D$, and $\varphi=\pi_{*}(z)$. The latter condition means that the eigenspaces of $\varphi(p)$ correspond to the two distinct values of $z$ lying above $p$, or in other words to the two points $p^{\prime}$ and $p^{\prime \prime}$. This being so, a nontrivial $\varphi$-invariant Hecke 
modification of $(E, \varphi)$ at the point $p$ comes from a transformation of $\mathcal{L}$ of the specific form

$$
\mathcal{L} \rightarrow \mathcal{L} \otimes \mathcal{O}\left(p^{\prime}-p^{\prime \prime}\right)
$$

for one or another of the two possible labelings of the two points $p^{\prime}$ and $p^{\prime \prime}$ lying above $p$. (This notion of a Hecke modification of a Higgs bundle $(E, \varphi)$ is mathematically natural and was taken as the starting point in [20].) When this is pushed down to $C$, it modifies $E$ in the desired fashion.

Now we can see why an $A$-brane $\mathcal{A}_{\boldsymbol{F}}$ supported on a fiber $\boldsymbol{F}$ of the Hitchin fibration and endowed with a flat line bundle $\mathcal{R}$ is a magnetic eigenbrane, that is an eigenbrane for the 't Hooft operator $T_{p}$. First of all, $T_{p}$ maps $\boldsymbol{F}$ to itself, since it preserves the characteristic polynomial of $\varphi$. Since $T_{p}$ preserves the support of $\mathcal{A}_{\boldsymbol{F}}$, it is conceivable for $\mathcal{A}_{\boldsymbol{F}}$ to be an eigenbrane for $T_{p}$.

Now, assuming that we choose $p$ so that $\varphi(p)$ is regular semi-simple (and we will only treat this case), the evaluation of $T_{p} \cdot \mathcal{A}_{\boldsymbol{F}}$ comes from a sum of contributions from the three $\varphi$-invariant Hecke modifications that were just described. One of them is the trivial Hecke modification, and this leaves $\mathcal{A}_{\boldsymbol{F}}$ invariant. The other two come from transformations $\mathcal{L} \rightarrow$ $\mathcal{L} \otimes \mathcal{O}\left(p^{\prime}-p^{\prime \prime}\right)$ (for some labeling of the two points). Such a transformation can be interpreted as an isomorphism $\Phi: \boldsymbol{F} \rightarrow \boldsymbol{F}$ of the Hitchin fiber. If the labeling of the two points $p^{\prime}$ and $p^{\prime \prime}$ is reversed, then $\Phi$ is replaced by $\Phi^{-1}$.

$\boldsymbol{F}$ is a complex torus, and $\Phi$ is a "translation" of $\boldsymbol{F}$ by a constant vector. In general, if $\mathcal{R} \rightarrow \boldsymbol{F}$ is a flat line bundle over a complex torus and $\Phi: \boldsymbol{F} \rightarrow \boldsymbol{F}$ is a translation, then $\Phi^{*}(\mathcal{R})=\mathcal{R} \otimes \mathcal{V}$ for some one-dimensional vector space $\mathcal{V}$. From this, it follows that $\mathcal{A}$ is an eigenbrane for $T_{p}$. In fact, we have

$$
T_{p} \cdot \mathcal{A}_{\boldsymbol{F}}=\mathcal{A}_{\boldsymbol{F}} \otimes\left(\mathbb{C} \oplus \mathcal{V} \oplus \mathcal{V}^{-1}\right),
$$

where the three contributions on the right come, respectively, from the trivial Hecke modification and the nontrivial modifications that involve $\Phi$ and $\Phi^{-1}$.

Let us compare this to what we had on the electric side. There, we considered a brane $\mathcal{B}$ whose support in $\mathcal{M}_{\mathrm{H}}\left(\mathrm{SO}_{3}\right)$ corresponds to a Higgs bundle $(E, \varphi)$. It obeyed $W_{p} \cdot \mathcal{B}=\mathcal{B} \otimes E_{p}$, where $E_{p}$ is the fiber at $p$ of $E$. Since we assume that $\varphi(p)$ is regular semi-simple (a property that is invariant under duality), we can decompose $E_{p}$ according to the eigenspaces of $\varphi(p) . \varphi(p)$ has two eigenspaces with nonzero eigenvalues; they are dual to each other because of the quadratic form on $E_{p}$, so we call them $\mathcal{X}$ and $\mathcal{X}^{-1}$. They have a natural isomorphism to $\mathcal{V}$ and $\mathcal{V}^{-1}$; this can be established by arguments similar to those used in demonstrating the geometric Langlands 
duality for $G L_{1}$. See [20] for a version of this calculation. The kernel of $\varphi(p)$ corresponds to the summand $\mathbb{C}$.

5.3.4. Interpretation What perhaps most needs clarification is the interpretation of the result just described.

That computation was made using the Hitchin fibration and other tools appropriate for the $B$-model in complex structure $I$. However, for the geometric Langlands program, we are really interested in $A$-branes for the $A$-model in symplectic structure $\omega_{K}$.

We observed earlier that the electric eigenbrane supported at a point is a brane of type $(B, B, B)$ - that is, it is a $B$-brane in each of complex structures $I, J$ and $K$. The dual statement is that the dual magnetic eigenbrane is a brane of type $(B, A, A)$. A brane of type $(B, A, A)$ is a brane that is simultaneously a $B$-brane in complex structure $I$ and an $A$-brane for the $A$-models of symplectic structure $\omega_{J}$ and $\omega_{K}$.

To explicitly see that a brane $\mathcal{A}_{\boldsymbol{F}}$ supported on a fiber $\boldsymbol{F}$ of the Hitchin fibration and endowed with a unitary flat line bundle $\mathcal{L}$ is of type $(B, A, A)$, we reason as follows. $\boldsymbol{F}$ is a complex Lagrangian submanifold in complex structure $I . \mathcal{A}_{\boldsymbol{F}}$ is a $B$-brane in complex structure $I$ because $\boldsymbol{F}$ is holomorphic in that complex structure, and a flat line bundle such as $\mathcal{L}$ is also holomorphic. $\boldsymbol{F}$ is Lagrangian for $\Omega_{I}=\omega_{J}+i \omega_{K}$, and hence is Lagrangian for $\omega_{J}$ and $\omega_{K}$. The most standard type of $A$-brane is a Lagrangian submanifold endowed with a unitary flat line bundle; $\mathcal{A}_{\boldsymbol{F}}$ qualifies whether we take the symplectic structure to be $\omega_{J}$ or $\omega_{K}$.

The dual of the fact that the Wilson operator $W_{p}$ preserves supersymmetry of type $(B, B, B)$ is that the 't Hooft operator $T_{p}$ preserves supersymmetry of type $(B, A, A)$. This statement means that if $\widetilde{\mathcal{A}}$ is a brane of type $(B, A, A)$ then so is $T_{p} \cdot \widetilde{\mathcal{A}}$. (More generally, if $\widetilde{\mathcal{A}}$ preserves any linear combination of these supersymmetries, then so does $T_{p} \cdot \widetilde{\mathcal{A}}$.) To actually identify which brane of type $(B, A, A)$ is $T_{p} \cdot \widetilde{\mathcal{A}}$, we can compute using whatever one of the supersymmetries is most convenient. Algebraic geometry is powerful, so it is likely to be convenient to compute $T_{p} \cdot \widetilde{\mathcal{A}}$ viewed as a $B$-brane in complex structure $I$. This is enough to determine $T_{p} \cdot \widetilde{\mathcal{A}}$ as a brane of type $(B, A, A)$, and in particular, as an $A$-brane of type $K$, which is what one wants for geometric Langlands.

The reason for the last statement is that given a $B$-brane of type $I$, there is at most one way to endow it with the structure of a brane of type $(B, A, A)$. Let us spell this out concretely in the present context. In Section 5.3.3, we used spectral curves and algebraic geometry to construct the brane $T_{p} \cdot \mathcal{A}_{\boldsymbol{F}}$ in terms of a holomorphic subvariety $\boldsymbol{F} \subset \mathcal{M}_{\mathrm{H}}\left(S L_{2}\right)$ and a holomorphic vector 
bundle $\mathcal{K} \rightarrow \boldsymbol{F}$. These data determine a $B$-brane in complex structure $I$. Endowing this brane with a structure of type $(B, A, A)$ means endowing $\mathcal{K}$ with a hermitian metric such that the induced unitary connection is flat; $\boldsymbol{F}$ with such a flat bundle is a brane of type $(B, A, A)$. The flat unitary connection with which a holomorphic bundle $\mathcal{K}$ can be so endowed is unique up to isomorphism if it exists. In the present context, because $T_{p}$ preserves supersymmetry of type $(B, A, A)$, we expect such a flat connection to exist, and the computation in Section 5.3.3 shows that it does.

A final comment concerns the "multiplier" $V_{p}$ in the formula expressing the fact that $\mathcal{A}_{\boldsymbol{F}}$ is a magnetic eigenbrane:

$$
T_{p} \cdot \mathcal{A}_{\boldsymbol{F}}=\mathcal{A}_{\boldsymbol{F}} \otimes V_{p}
$$

We want to discuss what happens when $p$ varies. In geometric Langlands, we expect that $V_{p}$ will be the fiber of a local system that is a flat vector bundle. That is not what we get in the most obvious way from the description based on the spectral curve and complex geometry in complex structure $I$. From that point of view, we obtain $V_{p}$ as the fiber of a rank 3 holomorphic vector bundle $V \rightarrow C$ (defined to begin with where $\varphi$ is regular semi-simple) that does not have any obvious flat structure. However, multiplication by the coordinate $z$ of the spectral curve gives a $K$-valued endomorphism that we will call $\theta$, so the multiplier is actually a Higgs bundle $(V, \theta)$. By solving Hitchin's equations, we associate with this Higgs bundle a rank 3 local system, and this we expect will be isomorphic to the $\mathrm{SO}_{3}$ local system with which we began.

5.3.5. Reducible fibers Now we are ready to consider the situation related to endoscopy. We consider a special fiber $\boldsymbol{F}$ of the Hitchin fibration that is a union of two irreducible components $\boldsymbol{F}_{1}$ and $\boldsymbol{F}_{2}$ that intersect each other on a divisor. This being so, we can construct rank $1 A$-branes $\mathcal{A}_{1}$ and $\mathcal{A}_{2}$ supported on $\boldsymbol{F}_{1}$ or $\boldsymbol{F}_{2}$. These branes are unique if $\boldsymbol{F}_{1}$ and $\boldsymbol{F}_{2}$ are simply connected, as in the case of a curve of genus 1 with 1 point of ramification; otherwise, they depend on parameters that we are not indicating explicitly.

In the derivation of Equation (5.28) describing the action of $T_{p}$, a key ingredient was the map $\Phi: \boldsymbol{F} \rightarrow \boldsymbol{F}$ by $\mathcal{L} \rightarrow \mathcal{L} \otimes \mathcal{O}\left(p^{\prime}-p^{\prime \prime}\right)$. The essential new fact in the case that $\boldsymbol{F}$ is reducible is simply that $\Phi$ exchanges the two component of $\boldsymbol{F}$. This was how we characterized the two components in Section 5.2.1. Likewise $\Phi^{-1}$ exchanges the two components. Hence $\Phi$ or $\Phi^{-1}$ exchange $\mathcal{A}_{1}$ and $\mathcal{A}_{2}$. Since $T_{p}$ acts by $1+\Phi+\Phi^{-1}$, it follows that we 
have up to isomorphism

$$
\begin{aligned}
& T_{p} \cdot \mathcal{A}_{1}=\mathcal{A}_{1}+2 \mathcal{A}_{2} \\
& T_{p} \cdot \mathcal{A}_{2}=\mathcal{A}_{2}+2 \mathcal{A}_{1} .
\end{aligned}
$$

This is in perfect parallel with formula (5.24) for the electric case.

If $\mathcal{A}_{1}$ and $\mathcal{A}_{2}$ have moduli, this should be described a little more precisely. $\mathcal{A}_{1}$ depends on the choice of a suitable line bundle $\mathcal{L} \rightarrow \boldsymbol{F}_{1}$, and we should take $\mathcal{A}_{2}$ to be the brane associated with the line bundle $\Phi^{*}(\mathcal{L}) \rightarrow \boldsymbol{F}_{2}$. Note that $\Phi^{*}(\mathcal{L})$ and $\left(\Phi^{-1}\right)^{*}(\mathcal{L})$ are isomorphic, though not canonically so.

One expects to get the more precise result analogous to (5.23) via the procedure of Section 5.3.4. One uses the standard methods of algebraic geometry to construct $T_{p} \cdot \mathcal{A}_{1}$ and $T_{p} \cdot \mathcal{A}_{2}$ as $B$-branes in complex structure I. This will give a result more precise than (5.30):

$$
\begin{aligned}
& T_{p} \cdot \mathcal{A}_{1}=\left(\mathcal{A}_{1} \otimes \mathcal{J}_{1}\right) \oplus\left(\mathcal{A}_{2} \otimes \mathcal{J}_{2}\right) \\
& T_{p} \cdot \mathcal{A}_{2}=\left(\mathcal{A}_{2} \otimes \mathcal{J}_{1}^{\prime}\right) \oplus\left(\mathcal{A}_{1} \otimes \mathcal{J}_{2}^{\prime}\right)
\end{aligned}
$$

with vector spaces $\mathcal{J}_{1}, \mathcal{J}_{2}$, etc., of dimensions indicated by the subscripts. All these admit natural $K$-valued endomorphisms $\theta_{1}, \theta_{2}$, etc., coming from the Higgs field (that is, multiplication by the coordinate $z$ of the spectral curve), and $\left(\mathcal{J}_{1}, \theta_{1}\right)$, etc., are Higgs bundles over $C$. Relating these Higgs bundles to local systems via Hitchin's equations, one expects to arrive at the analog of (5.23),

$$
\begin{aligned}
& T_{p} \cdot \mathcal{A}_{1}=\left(\left.\mathcal{A}_{2} \otimes U\right|_{p}\right) \oplus\left(\left.\mathcal{A}_{1} \otimes \operatorname{det} U\right|_{p}\right) \\
& T_{p} \cdot \mathcal{A}_{2}=\left(\left.\mathcal{A}_{1} \otimes U\right|_{p}\right) \oplus\left(\left.\mathcal{A}_{2} \otimes \operatorname{det} U\right|_{p}\right)
\end{aligned}
$$

5.3.6. The reciprocal case We can apply similar techniques to the reciprocal case ${ }^{\mathrm{L}} \mathrm{G}=\mathrm{SL}_{2}, \mathrm{G}=\mathrm{SO}_{3}$.

For gauge group $S L_{2}$, the basic Wilson operator to consider is the operator $\widetilde{W}_{p}$ associated with the two-dimensional representation. Roughly speaking, it acts by the obvious analog of Equation (5.17). Letting $(E, \widehat{\varphi})$ denote the universal Higgs bundle over $\mathcal{M}_{\mathrm{H}}\left(S L_{2}\right) \times C, \widetilde{W_{p}}$ acts on the sheaf $\mathcal{K}$ defining a $B$-brane $\mathcal{B}$ by

$$
\left.\mathcal{K} \rightarrow \mathcal{K} \otimes \mathrm{E}\right|_{p}
$$


where $\left.\mathrm{E}\right|_{p}$ is the restriction to $\mathcal{M}_{\mathrm{H}} \times p$ of the universal rank two bundle $\mathrm{E} \rightarrow \mathcal{M}_{\mathrm{H}} \times C . \widetilde{W}_{p}$ obeys

$$
\widetilde{W}_{p}^{2}=1+W_{p},
$$

expressing the fact that the tensor product of the two-dimensional representation with itself is a direct sum of the trivial representation and the three-dimensional representation; they correspond to the terms 1 and $W_{p}$ on the right-hand side of Equation (5.34).

An important subtlety reflects the fact that the center of $S L_{2}$ acts nontrivially in the two-dimensional representation. The universal bundle $E$ does not exist as a vector bundle in the usual sense. Rather, it must be understood as a twisted vector bundle, twisted by a certain $\mathbb{C}^{\times}$gerbe over $\mathcal{M}_{\mathrm{H}}$. The gerbe in question is induced from a $\mathbb{Z}_{2}$ gerbe $\left(\mathbb{Z}_{2}\right.$ being here the center of $S L_{2}$ ) by the inclusion $\mathbb{Z}_{2} \subset \mathbb{C}^{\times}$. As a result of the fact that $\left.\mathrm{E}\right|_{p}$ is a twisted vector bundle, the tensor product with it maps ordinary sheaves over $\mathcal{M}_{\mathrm{H}}$ to twisted ones, and vice versa. This means, in the language of [39], that the action of $\widetilde{W}_{p}$ on a brane shifts the discrete electric flux $e_{0}$, which is a character of the center of $S L_{2}$. The dual statement is that the dual 't Hooft operator $\widetilde{T}_{p}$ shifts the discrete magnetic flux $\boldsymbol{m}_{0}$, which is the second Stieffel-Whitney class $w_{2}$ of an $\mathrm{SO}_{3}$ bundle.

Roughly speaking, a skyscraper sheaf supported at a smooth point $r \in \mathcal{M}_{\mathrm{H}}\left(S L_{2}\right)$ gives an electric eigenbrane $\mathcal{B}$, just as in our earlier discussion for ${ }^{\mathrm{L}} \mathrm{G}=\mathrm{SO}_{3}$. But this is slightly oversimplified. The skyscraper sheaf supported at the point $r$ makes sense as either an ordinary sheaf or a twisted one, since the twisting involves a gerbe that is trivial when restricted to a smooth point. The tensor product with $E$ exchanges the two cases. So if we write $\mathcal{B}$ and $\mathcal{B}^{\prime}$ for the ordinary and twisted versions of the brane related to the skyscraper sheaf, then the action of the Wilson operator is

$$
\begin{aligned}
\widetilde{W}_{p} \cdot \mathcal{B} & =\left.\mathcal{B}^{\prime} \otimes \mathrm{E}\right|_{r \times p} \\
\widetilde{W}_{p} \cdot \mathcal{B}^{\prime} & =\left.\mathcal{B} \otimes \mathrm{E}\right|_{r \times p} .
\end{aligned}
$$

The sum $\widehat{\mathcal{B}}=\mathcal{B} \oplus \mathcal{B}^{\prime}$ is therefore an electric eigenbrane in the usual sense:

$$
\widetilde{W}_{p} \cdot \widehat{\mathcal{B}}=\left.\widehat{B} \otimes \mathrm{E}\right|_{r \times p} .
$$

The action of the dual 't Hooft operator $\widetilde{T}_{p}$ on an $S_{3}$ bundle $W$ or $\mathrm{SO}_{3}$ Higgs bundle $(W, \varphi)$ is quite similar to what has been described in Section 5.3.3. It is convenient to describe the action in terms of an $S L_{2}$ 
Higgs bundle $(E, \varphi)$ such that $W=\operatorname{ad}(E)$, where the degree of det $E$ is congruent mod 2 to $w_{2}(W)$. $E$ is not quite uniquely determined, but the following statements, when expressed in terms of $W=\operatorname{ad}(E)$, do not depend on the choice of $E$. Relative to some local decomposition of $E$ as a sum of line bundles $E=\mathcal{N}_{1} \oplus \mathcal{N}_{2}, \widetilde{T}_{p}$ acts by $E \rightarrow \mathcal{N}_{1}(p) \oplus \mathcal{N}_{2}$. This operation reverses the reduction mod 2 of the degree of det $E$, so it reverses $\boldsymbol{m}_{0}$, as expected.

If $\varphi=0$, the freedom to choose $\mathcal{N}_{1}$ leads to a family of possible Hecke modifications parametrized by $\mathbb{C P}^{1}$. This parameter space is compact (reflecting the fact that the two-dimensional representation of $S L_{2}$ is minuscule) so we do not need to add anything to compactify it. If $\varphi \neq 0, \widetilde{T}_{p}$ acts by a $\varphi$-invariant Hecke modification. If $\varphi(p)$ is regular semi-simple, this means that, in the last paragraph, we must take $\mathcal{N}_{1}$ to be one of the two eigenspaces of $\varphi$. The existence of two choices is dual to the fact that the representation of $S L_{2}$ that we started with is two-dimensional.

In terms of spectral curves, the action of a $\varphi$-invariant Hecke modification can be described very similar to Equation (5.27). The line bundle $\mathcal{L} \rightarrow D$ such that $E=\pi_{*}(\mathcal{L})$ transforms by

$$
\mathcal{L} \rightarrow \mathcal{L}\left(p^{*}\right),
$$

where $p^{*}$ may be either of the two points $p^{\prime}$ and $p^{\prime \prime}$ lying above $p$.

Now $\mathcal{M}_{\mathrm{H}}\left(S_{3}\right)$ has two components, classified by $w_{2}(W)$. We write $\boldsymbol{F}$ and $\boldsymbol{F}_{\theta}$ for the fibers of the Hitchin fibration for these two components. For $p^{*}$ equal to $p^{\prime}$ or $p^{\prime \prime},(5.37)$ corresponds to two maps $\Phi^{\prime}: \boldsymbol{F} \leftrightarrow \boldsymbol{F}_{\theta}$ and $\Phi^{\prime \prime}: \boldsymbol{F} \leftrightarrow \boldsymbol{F}_{\theta}$, each of which exchanges these two components. Acting on a brane supported on $\boldsymbol{F}$ with a flat bundle $\mathcal{L}, \widetilde{T}_{p}$ therefore gives a brane supported on $\boldsymbol{F}_{\theta}$ with flat bundle $\left(\Phi^{\prime}\right)^{*}(\mathcal{L}) \oplus\left(\Phi^{\prime \prime}\right)^{*}(\mathcal{L})$, and similarly with $\boldsymbol{F}$ and $\boldsymbol{F}_{\theta}$ exchanged. These are all branes of type $(B, A, A)$ and can be analyzed by arguments similar to those that we have already described. This leads to formulas dual to (5.35) if we consider branes supported on only one component, or to (5.36), if we form ordinary magnetic eigenbranes with support on both components.

5.3.7. The improper 't Hooft operator None of this gives a good example of geometric endoscopy, because an $S L_{2}$ local system cannot have a finite automorphism group that does not merely reduce to the center of $S L_{2}$. However, we can see endoscopy at work if we take $\widetilde{T}_{p}$ to act on the $A$-model not of $\mathcal{M}_{\mathrm{H}}\left(\mathrm{SO}_{3}\right)$ but of its cover. We recall that $\mathcal{M}_{\mathrm{H}}\left(\mathrm{SO}_{3}, 0\right)$ and $\mathcal{M}_{\mathrm{H}}\left(S_{3}, \theta\right)$ have covers (with covering group the finite abelian group $Q=$ $\left.H^{1}\left(C, \mathbb{Z}_{2}\right)\right)$ that are the proper and improper components of the $S L_{2}$ moduli 
space, $\mathcal{M}_{\mathrm{H}}\left(S L_{2}\right)$ and $\mathcal{M}_{\mathrm{H}}\left(S L_{2}^{*} ; p\right)$. The improper component depends on the choice of a basepoint $p \in C$, as explained in Section 5.2.3; it is convenient to take this to be the point $p$ at which we will apply the 't Hooft operator.

After lifting to the these covering spaces, the action of the 't Hooft operator $\widetilde{T}_{p}$ is much as we have already described. Mapping from $\mathcal{M}_{\mathrm{H}}\left(S L_{2}\right)$ to $\mathcal{M}_{\mathrm{H}}\left(S L_{2}^{*} ; p\right), \widetilde{T}_{p}$ tensors the line bundle on the spectral curve by $\mathcal{O}\left(p^{\prime}\right) \oplus$ $\mathcal{O}\left(p^{\prime \prime}\right)$; mapping from $\mathcal{M}_{\mathrm{H}}\left(S L_{2}^{*} ; p\right)$ to $\mathcal{M}_{\mathrm{H}}\left(S L_{2}\right)$, it tensors that line bundle by $\pi^{*}(\mathcal{O}(-p)) \otimes\left(\mathcal{O}\left(p^{\prime}\right) \oplus \mathcal{O}\left(p^{\prime \prime}\right)\right)=\mathcal{O}\left(-p^{\prime}\right) \oplus \mathcal{O}\left(-p^{\prime \prime}\right)$. These formulas are compatible with the fact that the two components parametrize, respectively, Higgs bundles $(E, \varphi)$ with $\operatorname{det} E=\mathcal{O}$ and $\operatorname{det} E=\mathcal{O}(p)$. Ensuring this has necessitated the prefactor $\pi^{*}(\mathcal{O}(-p))$ in one of the formulas.

As long as the spectral curve is smooth, the action of $\widetilde{T}_{p}$ on $A$-branes of $S L_{2}$, exchanging the two components, is similar to what we described earlier for $\mathrm{SO}_{3}$. Now, however, we can consider the case that the Hitchin fiber has two irreducible components. We write $\boldsymbol{F}_{1}$ and $\boldsymbol{F}_{2}$ for the two components of the special Hitchin fiber of $\mathcal{M}_{\mathrm{H}}\left(S L_{2}\right)$, and $\boldsymbol{F}_{1}^{*}$ and $\boldsymbol{F}_{2}^{*}$ for the two components of the special Hitchin fiber of $\mathcal{M}_{\mathrm{H}}\left(S L_{2}^{*} ; p\right)$. We likewise write $\mathcal{A}_{1}, \mathcal{A}_{2}$ and $\mathcal{A}_{1}^{*}, \mathcal{A}_{2}^{*}$ for $A$-branes of the usual type supported on these fibers.

In the action of the 't Hooft operator, the two operations of tensoring the line bundle on the spectral cover by $\mathcal{O}\left(p^{\prime}\right)$ and by $\mathcal{O}\left(p^{\prime \prime}\right)$ differ by the tensor product with $\mathcal{O}\left(p^{\prime}-p^{\prime \prime}\right)$. This is the basic operation that exchanges the two components. We can label the points to that $\mathcal{O}\left(p^{\prime}\right)$ maps $\boldsymbol{F}_{1}$ to $\boldsymbol{F}_{1}^{*}$ and $\boldsymbol{F}_{2}$ to $\boldsymbol{F}_{2}^{*}$, while $\mathcal{O}\left(p^{\prime \prime}\right)$ maps $\boldsymbol{F}_{1}$ to $\boldsymbol{F}_{2}^{*}$ and $\boldsymbol{F}_{2}$ to $\boldsymbol{F}_{1}^{*}$. (The tensor product with $\mathcal{O}\left(p^{\prime}\right)^{-1}$ or $\mathcal{O}\left(p^{\prime \prime}\right)^{-1}$ is of course the inverse operation.) There is no natural way to say which is which, since $p^{\prime}$ and $p^{\prime \prime}$ are exchanged by monodromy in $p$, and this monodromy similarly exchanges $\boldsymbol{F}_{1}^{*}$ and $\boldsymbol{F}_{2}^{*}$, as we noted at the end of Section 5.2.3.

The action of the 't Hooft operator $\widetilde{T}_{p}$ on branes $\mathcal{A}_{1,2}$ and $\mathcal{A}_{1,2}^{*}$ is schematically

$$
\begin{aligned}
& \widetilde{T}_{p} \cdot \mathcal{A}_{1}=\mathcal{A}_{1}^{*}+\mathcal{A}_{2}^{*} \\
& \widetilde{T}_{p} \cdot \mathcal{A}_{2}=\mathcal{A}_{1}^{*}+\mathcal{A}_{2}^{*},
\end{aligned}
$$

and similar formulas with $\mathcal{A}_{i}$ and $\mathcal{A}_{i}^{*}$ exchanged. These formulas and the analogous ones for the action of the 't Hooft operator $T_{p}$ dual to the threedimensional representation are compatible with the relation

$$
\widetilde{T}_{p}^{2}=1+T_{p}
$$

This relation is dual to Equation (5.34). 
Finally, let us discuss how natural is the operator $\widetilde{T}_{p}$. As an operator acting on branes on $\mathcal{M}_{\mathrm{H}}\left(S O_{3}\right)$, it is completely natural, being dual to the two-dimensional representation of the dual group ${ }^{\mathrm{L}} G=S L_{2}$. However, as an operation acting on branes on $\mathcal{M}_{\mathrm{H}}\left(S L_{2}\right)$, we cannot expect $\widetilde{T}_{p}$ to be entirely natural, since it is supposed to be dual to the two-dimensional representation, which does not exist as a representation of ${ }^{\mathrm{L}} \mathrm{G}=\mathrm{SO}_{3}$. The unnaturalness shows up in the fact that if we want $\widetilde{T}_{p}$ to act on branes on $\mathcal{M}_{\mathrm{H}}\left(S L_{2}\right)$, it maps them to branes on $\mathcal{M}_{\mathrm{H}}\left(S L_{2}^{*} ; p\right)$, a space whose definition depends on $p$, albeit relatively weakly. In contrast, for any reductive group $G$, 't Hooft operators associated with representations of ${ }^{\mathrm{L}} G$ always have a completely natural action on branes on $\mathcal{M}_{\mathrm{H}}(G)$.

As an operator on branes on the $S L_{2}$ moduli spaces, we call $\widetilde{T}_{p}$ the improper 't Hooft operator.

\section{Categories of eigensheaves}

In the previous sections, we have constructed $A$-branes on the moduli space of Higgs bundles which satisfy a property very similar to, but not quite the same, as the usual Hecke property. As explained in Section 4, to each of these $A$-branes, we should be able to attach a $\mathcal{D}$-module on $\operatorname{Bun}_{G}$. These $\mathcal{D}$-modules should then satisfy the same Hecke property. In this section, we explain the general framework in which we can interpret this property as a natural generalization of the standard notion of Hecke eigensheaf.

\subsection{Generalities on categories}

Let $H$ be a reductive algebraic group over $\mathbb{C}$ and $\operatorname{Rep}(H)$ the tensor category of finite-dimensional representations of $H$. We consider below two types of abelian categories associated to $H$.

(1) Categories equipped with an action of $H$. This means that any $h \in H$ gives rise to a functor $F_{h}$ on the category $\mathcal{C}$ sending each object $M$ of $\mathcal{C}$ to an object $F_{h}(M)$, and each morphism $f: M \rightarrow M^{\prime}$ to a morphism $F_{h}(f): F_{h}(M) \rightarrow F_{h}\left(M^{\prime}\right)$.

These functors have to satisfy natural compatibilities; in particular, for any $h, h^{\prime} \in H$ there is an isomorphism $i_{h, h^{\prime}}: F_{h h^{\prime}} \simeq F_{h} \circ F_{h^{\prime}}$, and we have $i_{h h^{\prime}, h^{\prime \prime}} i_{h, h^{\prime}}=i_{h, h^{\prime} h^{\prime \prime}} i_{h^{\prime}, h^{\prime \prime}}$.

Example: the category $\operatorname{Coh}(X)$ of coherent sheaves (or $B$-branes) on an algebraic variety $X$ equipped with an action of $H$. 
(2) Categories with a monoidal action of the tensor category $\operatorname{Rep}(H)$. This means that any object $V \in \operatorname{Rep}(H)$ defines a functor on $\mathcal{C}, M \mapsto$ $V \star M$, and these functors are compatible with the tensor structure on $\operatorname{Rep}(H)$.

Example: the category $\operatorname{Coh}(X / H)$ of coherent sheaves on the quotient $X / H$, where $X$ is as in point (1) above. ${ }^{33}$ Then for each $V \in$ $\operatorname{Rep}(H)$ we have a vector bundle $\mathcal{V}$ on $X / H$ associated with the principal $H$-bundle $X \rightarrow X / H$,

$$
\mathcal{V}=X \underset{H}{\times} V
$$

Its sheaf of sections (also denoted by $\mathcal{V}$ ) is an object of $\operatorname{Coh}(X / H)$. For any other object $M$ of $\operatorname{Coh}(X / H)$ we set

$$
V \star M:=\mathcal{V} \underset{\mathcal{O}_{X / H}}{\otimes} M
$$

One can pass between the categories of these two types:

The passage $(1) \longrightarrow(2)$ is called equivariantization;

The passage $(2) \longrightarrow(1)$ is called de-equivariantization.

These two procedures are inverse to each other. Here we only give a brief sketch as this material is fairly well-known [3].

Equivariantization is done as follows: given a category $\mathcal{C}$ of type $(1)$, we construct a new category $\mathcal{C}^{\prime}$ of type $(2)$. Its objects are the data $\left(M,\left(\mu_{h}\right)_{h \in H}\right)$, where $M \in \mathcal{C}$ and

$$
\mu_{h}: F_{h}(M) \stackrel{\sim}{\longrightarrow} M, \quad h \in H,
$$

is a collection of isomorphisms such that $\mu_{h h^{\prime}}=\mu_{h} \circ \mu_{h^{\prime}}$. Given $V \in \operatorname{Rep}(H)$, we define a new object of $\mathcal{C}^{\prime}$,

$$
V \star\left(M,\left(\mu_{h}\right)_{h \in H}\right)=\left(\underline{V} \otimes_{\mathbb{C}} M,\left(\rho(h) \otimes \mu_{h}\right)_{h \in H}\right),
$$

where $\underline{V}$ is the vector space underlying $V$, and $\rho: H \rightarrow \operatorname{End}(\underline{V})$ is the representation of $H$ on $V$. Thus, we see that $\operatorname{Rep}(H)$ acts on $\mathcal{C}^{\prime}$.

Example: if $\mathcal{C}=\operatorname{Coh}(X)$, then $\mathcal{C}^{\prime}$ is the category of $H$-equivariant coherent sheaves on $X$ which is the same as the category $\operatorname{Coh}(X / H)$ of coherent sheaves on $X / H$.

\footnotetext{
${ }^{33}$ Here we may suppose for simplicity that this action is free, but this is not necessary if we are willing to consider $X / H$ as an algebraic stack - or an orbifold, in the case of a finite group $H$.
} 
De-equivariantization is similar, and this is where the notion of "Hecke eigenobject" naturally appears.

Given a category $\mathcal{C}^{\prime}$ of type (2), we define a new category $\mathcal{C}$ of type $(1)$. Its objects are the data $\left(M,\left(\alpha_{V}\right)_{V \in \operatorname{Rep}(H)}\right)$, where $M \in \mathcal{C}^{\prime}$ and

$$
\alpha_{V}: V \star M \stackrel{\sim}{\longrightarrow} \underline{V} \otimes_{\mathbb{C}} M, V \in \operatorname{Rep}(H),
$$

is a collection of isomorphisms compatible with the tensor product structure on $\operatorname{Rep}(H)$. We will call $\left(M,\left(\alpha_{V}\right)_{V \in \operatorname{Rep}(H)}\right)$ a Hecke eigenobject of the category $\mathcal{C}^{\prime}$.

The group $H$ naturally acts on $\mathcal{C}$ : for $h \in H$, we define

$$
F_{h}\left(\left(M,\left(\alpha_{V}\right)_{V \in \operatorname{Rep}(H)}\right)\right)=\left(M,\left((\rho(h) \otimes \mathrm{id}) \circ \alpha_{V}\right)_{V \in \operatorname{Rep}(H)}\right) .
$$

In other words, $M$ stays the same, but we twist the isomorphism $\alpha_{V}$ by $h$. Thus, $\mathcal{C}$ is indeed a category of type (1).

\subsection{Examples}

The simplest example of a category of type (2) is the category $\operatorname{Rep}(H)$ equipped with the natural monoidal action on itself. Consider the corresponding de-equivariantized category $\mathcal{C}^{\prime}$. Let $\mathcal{O}_{H}$ be the algebra of functions on $H$, that is, the regular representation of $H$. We have an isomorphism

$$
\mathcal{O}_{H}=\bigoplus_{V \in \operatorname{Irrep}(H)} V \otimes V^{*}
$$

respecting both left and right actions of $H$ on the two sides (here $\operatorname{Irrep}(H)$ is the set of equivalence classes of irreducible representations of $H$, and for each representation $V$, we denote by $V^{*}$ the dual representation).

The data of the isomorphisms $\alpha_{V}$ in Equation (6.1) and the compatibilities between them may be neatly summarized as the structure of an $\mathcal{O}_{H}$-module on $M$, compatible with the left action of $H$. Thus, $\mathcal{C}^{\prime}$ is the category of $H$-equivariant coherent sheaves on $H$. This category is equivalent to the category Vect of vector spaces. Indeed, we have a functor $G$ : Vect $\rightarrow \mathcal{C}^{\prime}$ sending $U \in$ Vect to $U \otimes \mathcal{O}_{H}$. Its quasi-inverse functor $\mathcal{C}^{\prime} \rightarrow$ Vect is defined by sending $\mathcal{M} \in \mathcal{C}^{\prime}$ to its fiber at $1 \in H$. The natural action of $H$ on $\mathcal{C}^{\prime}$ described above becomes the trivial action on Vect. The corresponding $H$-equivariant category of Vect has as its objects vector spaces equipped with an action of $H$. Thus, by equivariantizing $\mathcal{C}^{\prime}$, we recover the category $\operatorname{Rep}(H)$ we started with, as promised. 
It is useful to consider the case when $H=\mathbb{Z}_{2}=\{1,-1\}$ in more concrete terms. In this case $\operatorname{Rep}(H)$ has two irreducible one-dimensional representations: the trivial one, $I$, and the sign representation, $S$.

Let $\mathcal{C}$ be the following category of type (2): it has two irreducible objects $A_{+}$and $A_{-}$, and the $\operatorname{Rep}\left(\mathbb{Z}_{2}\right)$ acts as follows:

$$
I \star A_{ \pm}=A_{ \pm}, \quad S \star A_{ \pm}=A_{\mp} .
$$

All other objects of $\mathcal{C}$ are direct sums of copies of $A_{+}$and $A_{-}$.

Let us assume first that there is a preferred object among $A_{+}$and $A_{-}$ corresponding to the trivial representation, say, $A_{+}$.

What does the corresponding category $\mathcal{C}^{\prime}$ of type (1) look like? By definition, its objects have the form $\left(M,\left(\alpha_{I}, \alpha_{S}\right)\right)$, where $M \in \mathcal{C}$ and

$$
\alpha_{I}: I \star M \simeq M, \quad \alpha_{S}: S \star M \simeq M
$$

are isomorphisms satisfying the following conditions. First of all, $I \star M$ is $M$ and $\alpha_{I}$ is the identity map $M \rightarrow M$. Second,

$$
\left(\alpha_{S}\right)^{2}: M=I \star M=(S \otimes S) \star M=S \star(S \star M) \rightarrow S \star M \rightarrow M
$$

is the identity. Thus, our data may be recorded as pairs $(M, \alpha)$, where $M \in \mathcal{C}$ and $\alpha=\alpha_{S}$ is an isomorphism $S \star M \rightarrow M$ such that $\alpha^{2}=$ id.

It is easy to see that if $M$ is an object of $\mathcal{C}$ such that there exists $\alpha: S \star$ $M \simeq M$ satisfying the above property, then $M$ is a direct sum of copies of

$$
A:=A_{+} \oplus A_{-} .
$$

Let us look at objects of $\mathcal{C}^{\prime}$ of the form $(A, \alpha)$. We have $S \star A=S\left(A_{+}\right) \oplus$ $S\left(A_{-}\right)=A$. Therefore, $\alpha: A \rightarrow A$ is determined by two nonzero scalars $\epsilon_{ \pm}$ corresponding to the action of $\alpha$ on $A_{ \pm}$(we have assumed that both $A_{+}$and $A_{-}$are irreducible and nonisomorphic). The condition that $\alpha^{2}=$ id implies that $\epsilon_{+} \epsilon_{-}=1$. Given two such objects, $\left(A,\left(\epsilon_{+}, \epsilon_{-}\right)\right)$and $\left(A,\left(\epsilon_{+}^{\prime}, \epsilon_{-}^{\prime}\right)\right)$, an isomorphism between them is a pair of nonzero scalars $\left(\lambda_{+}, \lambda_{-}\right)$acting on $A_{+}$and $A_{-}$such that $\epsilon_{+} / \epsilon_{+}^{\prime}=\lambda_{+} / \lambda_{-}$. Thus, $\lambda_{+}$is determined by $\lambda_{-}$and vice versa.

Thus, the category $\mathcal{C}^{\prime}$ is very simple: up to an isomorphism there is a unique irreducible object, $A$, and all other objects are isomorphic to a direct sum of copies of this object. In fact, $\mathcal{C}^{\prime}$ is equivalent to the category Vect of vector spaces. The functor $\mathcal{C}^{\prime} \rightarrow$ Vect sends $M \in \mathcal{C}^{\prime}$ to $\operatorname{Hom}\left(A_{+}, M\right)$.

In the study of geometric endoscopy for $S L_{2}$, we encounter a category of $A$-branes similar to the category $\mathcal{C}$. It also has two irreducible objects, 
$\mathcal{A}_{+}$and $\mathcal{A}_{-}$, and $\operatorname{Rep}\left(\mathbb{Z}_{2}\right)$ acts on it as in formula (6.3). However, there is no preferred object among $\mathcal{A}_{+}$and $\mathcal{A}_{-}$; in other words, there is no canonical equivalence between $\mathcal{C}$ and $\operatorname{Rep}\left(\mathbb{Z}_{2}\right)$. It is natural to ask how this ambiguity is reflected in the category $\mathcal{C}^{\prime}$.

The answer is clear: the category $\mathcal{C}^{\prime}$ is still equivalent to Vect, but there are two such equivalences and there is no way to choose one of them over the other. The corresponding functors $\mathcal{C}^{\prime} \rightarrow \operatorname{Vect}$ send $M \in \mathcal{C}^{\prime}$ to $\operatorname{Hom}\left(\mathcal{A}_{ \pm}, M\right)$. Under both of these equivalences all objects $\left(A,\left(\epsilon_{+}, \epsilon_{-}\right)\right)$, with $\epsilon_{+} \epsilon_{-}=1$, of $\mathcal{C}^{\prime}$ go to the one-dimensional vector space $\mathbb{C}$ (viewed as an object of Vect), but the isomorphism

$$
\left(A,\left(\epsilon_{+}, \epsilon_{-}\right)\right) \rightarrow\left(A,\left(\epsilon_{+}^{\prime}, \epsilon_{-}^{\prime}\right)\right)
$$

given by $\left(\lambda_{+}, \lambda_{-}\right)$, as above, goes either to the isomorphism $\mathbb{C} \rightarrow \mathbb{C}$ given multiplication by $\lambda_{+}$or by $\lambda_{-}$.

Thus, to each of the two objects, $\mathcal{A}_{+}$and $\mathcal{A}_{-}$, corresponds a particular equivalence $\mathcal{C}^{\prime} \simeq$ Vect, but inasmuch as we cannot choose between $\mathcal{A}_{+}$and $\mathcal{A}_{-}$, we cannot choose one of these equivalences over the other (it is easy to see that these equivalences are not isomorphic to each other as functors $\mathcal{C}^{\prime} \rightarrow$ Vect). This is what replaces the ambiguity between $\mathcal{A}_{+}$and $\mathcal{A}_{-}$in the category $\mathcal{C}$ of type $(2)$ when we pass to the category $\mathcal{C}^{\prime}$ of type $(1)$.

The existence of two different equivalences of categories $\mathcal{C}^{\prime} \simeq$ Vect certainly looks like a more subtle and esoteric notion than the more concrete notion that the category $\mathcal{C}$ has two indistinguishable objects. This is a good illustration of why, from the practical point of view, it is often better to work with a category of type (2) than with a category of type (1).

The point is, however, that the two descriptions are equivalent to each other. To convince ourselves of that it is instructive to see how we can recover $\mathcal{C}$ from $\mathcal{C}^{\prime}$. Let us apply the equivariantization procedure to $\mathcal{C}^{\prime}$. Hence we define a new category $\mathcal{C}^{\prime \prime}$, whose objects are pairs $(M, \mu)$, where $M$ is an object of $\mathcal{C}^{\prime}$ and $\mu=\mu_{-1}$ is an isomorphism between $M$ and the new object $M^{\prime}$ obtained by applying the functor corresponding to $-1 \in \mathbb{Z}_{2}$ to $M$. Note that $\mu_{1}=\mathrm{id}: M \rightarrow M$ and so we must have

$$
\mu^{2}=\mathrm{id}
$$

to satisfy the relations of $\mathbb{Z}_{2}$. If $M=\left(\mathcal{A},\left(\epsilon_{+}, \epsilon_{-}\right)\right)$, then $M^{\prime}=\left(\mathcal{A},\left(-\epsilon_{+},-\epsilon_{-}\right)\right)$, and we have exactly two isomorphisms $\mu_{ \pm}$between $M$ and $M^{\prime}$, satisfying $\mu^{2}=\mathrm{id}$ : one acts by \pm 1 on $\mathcal{A}_{ \pm} \subset \mathcal{A}$, and the other acts as $\mp 1$. Thus, we see that there are two nonisomorphic objects in $\mathcal{C}^{\prime \prime}$, which correspond under 
a canonical equivalence $\mathcal{C}^{\prime \prime} \simeq \mathcal{C}$ to $\mathcal{A}_{+}$and $\mathcal{A}_{-}$, respectively. In fact, it is instructive to think of $\frac{1}{2}\left(1+\mu_{ \pm}\right)$as a projector onto $\mathcal{A}_{ \pm}$in $A$.

\subsection{Hecke eigensheaves}

The reason why we have discussed all these subtleties in such great detail is that the category that most closely matches the usual notion of Hecke eigenfunctions of the classical theory of automorphic forms is a category of type (1), so to understand fully the connection to the classical theory of automorphic forms we have to go through a category of type (1). However, the category of $A$-branes considered in Section 5 is naturally a category of type (2). Therefore, we have to make a link between the two types of categories.

Let us recall the traditional definition of Hecke eigensheaves used in the geometric Langlands program (see, e.g., [8] or [25, Section 6.1]). These are $\mathcal{D}$-modules on $\operatorname{Bun}_{G}$, the moduli stack of $G$-bundles on a curve $C$, satisfying the Hecke eigenobject property. To explain this more precisely, recall that for each finite-dimensional representation $V$ of the dual group ${ }^{\mathrm{L}} G$, we have a Hecke functor $H_{V}$ acting from the category of $\mathcal{D}$-modules on $\operatorname{Bun}_{G}$ to the category of $\mathcal{D}$-modules on $C \times \operatorname{Bun}_{G}$. We will not recall the definition of these functors here, referring the reader to $[8,25]$. We note that these functors are closely related to the 't Hooft operators discussed in Section 5.3, as explained in [39].

Let $\mathcal{E}$ be a flat ${ }^{\mathrm{L}} G$-bundle on $C .{ }^{34}$ A Hecke eigensheaf with "eigenvalue" $\mathcal{E}$ is by definition a collection of data

$$
\left(\mathcal{F},\left(\alpha_{V}\right)_{V \in \operatorname{Rep}\left({ }^{\mathrm{L}} G\right)}\right),
$$

where $\mathcal{F}$ is a $\mathcal{D}$-module on $\operatorname{Bun}_{G}$ and $\left(\alpha_{V}\right)$ is a collection of isomorphisms

$$
\alpha_{V}: H_{V}(\mathcal{F}) \stackrel{\sim}{\longrightarrow} V_{\mathcal{E}} \otimes \mathcal{F}
$$

where

$$
V_{\mathcal{E}}=\mathcal{E} \underset{{ }{ }_{G}}{\times} V
$$

is the flat vector bundle on $C$ associated to $V$.

These isomorphisms must satisfy natural compatibility conditions with respect to the composition of the Hecke functors $H_{V}$ on the LHS and the

\footnotetext{
${ }^{34}$ Here we consider for simplicity the unramified case, but the definition is easily generalized to the ramified case; we simply omit the points of ramification of $\mathcal{E}$.
} 
tensor product of representation on the RHS of (6.5), as well as the natural associativity condition. ${ }^{35}$

Let $\mathcal{A} u t_{\mathcal{E}}$ be the category of all Hecke eigensheaves with eigenvalue $\mathcal{E}$. To make contact with the categories of type (1) studied in the previous section, let us fix a point $x \in C$. Then the restriction of the Hecke operator $H_{V}$ to $x$ is a functor $H_{V, x}$ from the category of $\mathcal{D}$-modules on $\operatorname{Bun}_{G}$ to itself. For a Hecke eigensheaf (6.4), by restricting the isomorphisms $\alpha_{V}$ to $x$, we obtain a compatible collection of isomorphisms

$$
\alpha_{V, x}: H_{V, x}(\mathcal{F}) \stackrel{\sim}{\longrightarrow} V_{\mathcal{E}, x} \otimes \mathcal{F}
$$

Here

$$
V_{\mathcal{E}, x}=\mathcal{E}_{x} \underset{\mathrm{L} G}{\times} V,
$$

where $\mathcal{E}_{x}$ is the fiber of $\mathcal{E}$ at $x$, is a vector space isomorphic to $V$. The data of $\mathcal{F}$ and $\left.\left(\alpha_{V, x}\right)_{V \in \operatorname{Rep}\left({ }^{\mathrm{L}} G\right)}\right)$ are precisely the kind of data that we used above in the definition of the de-equivariantized category. A Hecke eigensheaf (6.4) is therefore an object of this type, except that instead of just one collection of Hecke isomorphisms (6.1) we have an entire family of such collections parametrized by points of the curve $C$. The condition (6.5) actually contains much more information than the data of the isomorphisms (6.6) for all $x \in C$, because formula (6.5) also describes the dependence of the "eigenvalues" $V_{\mathcal{E}, x}$ of the Hecke operators on $x$ : they "vary" according to the local system $\mathcal{E}$.

If we only impose condition (6.6) (for a particular $x \in C$ ), then the corresponding category carries an action of the group ${ }^{\mathrm{L}} G$, or, more precisely, its twist by the ${ }^{\mathrm{L}} G$-torsor $\mathcal{E}_{x}$, that is, ${ }^{\mathrm{L}} G_{\mathcal{E}_{x}}=\mathcal{E}_{x}{ }_{{ }^{\mathrm{L}} G}{ }^{\mathrm{L}} G$. This action is defined as in formula (6.2). If, on the other hand, we impose the full Hecke condition (6.1), then the group ${ }^{\mathrm{L}} G_{\mathcal{E}_{x}}$ no longer acts. Rather, we have an action of the (global) group of automorphisms of $\mathcal{E}$, that is, $\Gamma\left(C,{ }^{\mathrm{L}} G_{\mathcal{E}}\right)$, where

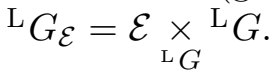

Suppose that the group of automorphisms of our local system $\mathcal{E}$ is trivial. This means, in particular, that the center of ${ }^{\mathrm{L}} G$ is trivial, so that ${ }^{\mathrm{L}} G$ is a semi-simple group of adjoint type. In this case, it is expected that there is a unique irreducible $\mathcal{D}$-module $\mathcal{F}$ satisfying the Hecke property $(6.1)$, and all other $\mathcal{D}$-modules satisfying this property are direct sums of its copies. If so, then there is essentially a unique way to construct the isomorphisms $\alpha_{V}$

\footnotetext{
${ }^{35}$ We will see in Section 8.2 that an additional commutativity condition needs to be imposed on the isomorphisms $\alpha_{V}$.
} 
in (6.5) for the irreducible $\mathcal{D}$-modules $\mathcal{F}$, and this is why in this case one usually suppresses the data of $\left(\alpha_{V}\right)$.

However, these data become important in the case when $\mathcal{E}$ has a nontrivial group of automorphisms

$$
\Gamma:=\Gamma\left(C,{ }^{\mathrm{L}} G_{\mathcal{E}}\right)
$$

Then we define an action of the group $\Gamma$ on the category $\mathcal{A} u t_{\mathcal{E}}$ as in formula (6.2). Namely, given an object $\left(\mathcal{F},\left(\alpha_{V}\right)\right)$ of the category $\mathcal{A} u t_{\mathcal{E}}$ as in formula (6.4), we construct a new object $\left(\mathcal{F},\left(\alpha_{V}^{s}\right)\right)$, where

$$
\alpha_{V}^{s}=(s \otimes 1) \circ \alpha_{V} .
$$

This new object may not be isomorphic to the old one (and even if it is, it may be isomorphic to it in different ways).

\subsection{Category of Hecke eigensheaves in the endoscopic example}

Let us discuss the category of Hecke eigensheaves in our endoscopic example, when $G=S L_{2},{ }^{\mathrm{L}} G=S O_{3}$ and $\Gamma=\mathbb{Z}_{2}$. We expect that in this case any $\mathcal{D}$-module satisfying the Hecke eigensheaf property is a direct sum of copies of a $\mathcal{D}$-module, which we will denote by $\mathcal{F}$. As explained in Sections 2.3 and 4 , the $\mathcal{D}$-module $\mathcal{F}$ corresponds to an $A$-brane $\mathcal{A}$ on a singular fiber of $\mathcal{M}_{\mathrm{H}}(G)$, which is a magnetic eigenbrane with respect to the 't Hooft operators. As we explained in Section 5, this $A$-branes is reducible:

$$
\mathcal{A}=\mathcal{A}_{+} \oplus \mathcal{A}_{-},
$$

where the $A$-branes $\mathcal{A}_{ \pm}$are irreducible. Furthermore, there is not a preferred one among them (see Section 10 for a more detailed discussion of this point). Actually, because of this ambiguity, we previously used the notation $\mathcal{A}_{1}$ and $\mathcal{A}_{2}$ for these $A$-branes, in order to emphasize that they do not correspond canonically to the $B$-branes $\mathcal{B}_{+}$and $\mathcal{B}_{-}$. But from now on we will use the notation $\mathcal{A}_{ \pm}$.

Therefore, we expect that the $\mathcal{D}$-module $\mathcal{F}$ is also reducible:

$$
\mathcal{F}=\mathcal{F}_{+} \oplus \mathcal{F}_{-}
$$

and each $\mathcal{F}_{ \pm}$is irreducible. We also expect that neither of them is preferred over the other one. 
Recall that the notion of an eigensheaf, as defined above, includes the isomorphisms $\alpha_{V}$ for all representations $V$ of $S_{3}$. By using the compatibility with the tensor product structure, we find that everything is determined by the adjoint representation of $\mathrm{SO}_{3}$, which we denote by $W$, as before. A Hecke eigensheaf may therefore be viewed as a pair $(\mathcal{F}, \alpha)$, where

$$
\alpha: H_{W}(\mathcal{F}) \stackrel{\sim}{\longrightarrow} W_{\mathcal{E}} \otimes \mathcal{F}
$$

In the endoscopic case, the structure group of our $\mathrm{SO}_{3}$-local system $\mathcal{E}$ is reduced to the subgroup

$$
O_{2}=\mathbb{Z}_{2} \ltimes \mathbb{C}^{\times}
$$

(but not to a smaller subgroup). Denote by $U$ the defining two-dimensional representation of $\mathrm{O}_{2}$. Then $\operatorname{det} U$ is the one-dimensional sign representation induced by the homomorphism $\mathrm{O}_{2} \rightarrow \mathbb{Z}_{2}$. We have

$$
W=(\operatorname{det} U \otimes I) \oplus(U \otimes S),
$$

as a representation of $\mathrm{O}_{2} \times \mathbb{Z}_{2}$, where $\mathbb{Z}_{2}$ is the centralizer of $\mathrm{O}_{2}$ in $\mathrm{SO}_{3}$ (which coincides with the center of $\mathrm{O}_{2}$ ), and, as before, $S$ is the sign representation of $\mathbb{Z}_{2}$, and $I$ is the trivial representation of $\mathbb{Z}_{2}$. Therefore we have the following decomposition of the corresponding local system:

$$
W_{\mathcal{E}}=\left(\operatorname{det} U_{\mathcal{E}} \otimes I\right) \oplus\left(U_{\mathcal{E}} \otimes S\right)
$$

We may twist the isomorphism $\alpha$ by the action of the nontrivial element $\tau \in \mathbb{Z}_{2}=\Gamma$, which is in the group of automorphisms of our local system. This way we obtain a new Hecke eigensheaf, that is, a pair $\left(\mathcal{F}, \alpha^{\prime}\right)$, where $\alpha^{\prime}=(\tau \otimes 1) \circ \alpha$. The objects $(\mathcal{F}, \alpha)$ and $\left(\mathcal{F}, \alpha^{\prime}\right)$ of $\mathcal{A} u t_{\mathcal{E}}$ are isomorphic, but noncanonically. There are in fact two natural isomorphisms, equal to \pm 1 on $\mathcal{F}_{ \pm}$or $\mp 1$ on $\mathcal{F}_{ \pm}$, and there is no natural way to choose between them.

We expect that any object of the category $\mathcal{A} u t_{\mathcal{E}}$ of Hecke eigensheaves is isomorphic to a direct sum of copies of $(\mathcal{F}, \alpha)$. As in the toy model discussed in Section 6.2, this means that the category $\mathcal{A} u t_{\mathcal{E}}$ is equivalent to the category of vector spaces, but in two different ways, corresponding to the choice of $\mathcal{F}_{+}$and $\mathcal{F}_{-}$.

\subsection{Fractional Hecke eigensheaves}

Next, we introduce a category of Hecke eigensheaves of type (2). 
Suppose again that we are given an ${ }^{\mathrm{L}} G$-local system $\mathcal{E}$ on a curve $C$, and let $\Gamma$ be the group of its automorphisms. To simplify our discussion below, we will identify $\Gamma$ with a subgroup of ${ }^{\mathrm{L}} G$ by picking a point $x \in C$ and choosing a trivialization of the fiber $\mathcal{E}_{x}$ of $\mathcal{E}$ at $x$. (This allows us to assign to $\mathcal{E}$ a homomorphism $\pi_{1}(C, x) \rightarrow{ }^{\mathrm{L}} G$. The group $\Gamma$ may then be defined as the centralizer of its image.)

Suppose that we are given an abelian subcategory $\mathcal{C}$ of the category of $\mathcal{D}$-modules on $\operatorname{Bun}_{G}$ equipped with an action of the tensor category $\operatorname{Rep}(\Gamma)$. In other words, for each $R \in \operatorname{Rep}(\Gamma)$, we have a functor

$$
M \mapsto R \star M,
$$

and these functors compose in a way compatible with the tensor product structure on $\operatorname{Rep}(\Gamma)$. The category of Hecke eigensheaves of type (2) with "eigenvalue" $\mathcal{E}$ will have as objects the following data:

$$
\left(\mathcal{F},\left(\alpha_{V}\right)_{V \in \operatorname{Rep}\left({ }^{\mathrm{L}} G\right)}\right),
$$

where $\mathcal{F}$ is an object of $\mathcal{C}$, and the $\alpha_{V}$ are isomorphisms defined below.

Denote by $\operatorname{Res}_{\Gamma}(V)$ the restriction of a representation $V$ of ${ }^{\mathrm{L}} G$ to $\Gamma$. If $\operatorname{Rep}(\Gamma)$ is a semi-simple category (which is the case, for example, if $\Gamma$ is a finite group), then we obtain a decomposition

$$
\operatorname{Res}_{\Gamma}(V)=\bigoplus_{i} F_{i} \otimes R_{i}
$$

where the $R_{i}$ are irreducible representations of $\Gamma$ and $F_{i}$ is the corresponding representation of the centralizer of $\Gamma$ in ${ }^{\mathrm{L}} G$. Twisting by $\mathcal{E}$, we obtain a local system $\left(\operatorname{Res}_{\Gamma}(V)\right)_{\mathcal{E}}$ on $C$ with a commuting action of $\Gamma$, which decomposes as follows:

$$
\left(\operatorname{Res}_{\Gamma}(V)\right)_{\mathcal{E}}=\bigoplus_{i}\left(F_{i}\right)_{\mathcal{E}} \otimes R_{i} .
$$

Note that since $\Gamma$ is the group of automorphisms of $\mathcal{E}$, the structure group of $\mathcal{E}$ is reduced to the centralizer of $\Gamma$ in $G$, and $F_{i}$ is a representation of this centralizer. Therefore, $F_{i}$ may be twisted by $\mathcal{E}$, and the resulting local system (or flat vector bundle) on $C$ is denoted by $\left(F_{i}\right)_{\mathcal{E}}$.

The isomorphisms $\alpha_{V}$ have the form

$$
\alpha_{V}: H_{V}(M) \stackrel{\sim}{\longrightarrow}\left(\operatorname{Res}_{\Gamma}(V)\right)_{\mathcal{E}} \star M=\bigoplus_{i}\left(F_{i}\right)_{\mathcal{E}} \otimes\left(R_{i} \star M\right), \quad M \in \mathcal{C}
$$


and they have to be compatible in the obvious sense. We will denote the category with objects (6.9) satisfying the above conditions by $\mathcal{A} u t_{\mathcal{E}}^{\prime} \cdot{ }^{36}$

The category of Hecke eigensheaves of type (2) has the advantage of being more concrete than the category of type (1), and it matches more closely the structure of the categories of $A$ - and $B$-branes that we have found in Section 5 .

However, the category $\mathcal{A} u t_{\mathcal{E}}$ may be reconstructed from $\mathcal{A} u t_{\mathcal{E}}^{\prime}$ by the procedure of de-equivariantization along the lines of Section 6.1. Conversely, applying the procedure of equivariantization to $\mathcal{A} u t_{\mathcal{E}}$, we obtain a category that is equivalent to $\mathcal{A} u t_{\mathcal{E}}^{\prime}$.

What does the category $\mathcal{A} u t_{\mathcal{E}}^{\prime}$ look like in our main example of geometric endoscopy? In this case, the category $\mathcal{C}$ should have two irreducible objects, $\mathcal{F}_{+}$and $\mathcal{F}_{-}$, which are the $\mathcal{D}$-modules on $\operatorname{Bun}_{G}$ corresponding to the fractional $A$-branes $\mathcal{A}_{+}$and $\mathcal{A}_{-}$. The category $\operatorname{Rep}\left(\mathbb{Z}_{2}\right)$ acts on them as follows: the sign representation $S$ of $\Gamma=\mathbb{Z}_{2}$ permutes them,

$$
S \star \mathcal{F}_{ \pm}=\mathcal{F}_{\mp}
$$

while the trivial representation $I$ acts identically.

Since the category of representations of $\mathrm{SO}_{3}$ is generated by the adjoint representation $W$, it is sufficient to formulate the Hecke property (6.10) only for the adjoint representation $\mathrm{W}$ of $\mathrm{SO}_{3}$. It reads

$$
\begin{aligned}
& H_{W}\left(\mathcal{F}_{+}\right) \simeq\left(\operatorname{det} U_{\mathcal{E}} \otimes \mathcal{F}_{+}\right) \oplus\left(U_{\mathcal{E}} \otimes \mathcal{F}_{-}\right), \\
& H_{W}\left(\mathcal{F}_{-}\right) \simeq\left(\operatorname{det} U_{\mathcal{E}} \otimes \mathcal{F}_{-}\right) \oplus\left(U_{\mathcal{E}} \otimes \mathcal{F}_{+}\right),
\end{aligned}
$$

where $\operatorname{det} U_{\mathcal{E}}$ and $U_{\mathcal{E}}$ are the summands of $W_{\mathcal{E}}$ defined in formula (6.8). This matches the action of the 't Hooft operators on the $A$-branes given by formula (5.32). Since that formula describes the behavior of the fractional branes $\mathcal{A}_{ \pm}$, we will call the property expressed by formulas (6.10) and (6.11) the fractional Hecke property, and the corresponding $\mathcal{D}$-modules fractional Hecke eigensheaves. On the other hand, we will call the ordinary Hecke property (6.5) the regular Hecke property and the $\mathcal{D}$-modules satisfying it regular Hecke eigensheaves (the reason for this terminology is that such an eigensheaf corresponds to the regular representation of the group $\Gamma$ ).

\footnotetext{
${ }^{36}$ As we will see in Section 8.2, we also need to impose an additional commutativity condition on the isomorphisms $\alpha_{V}$.
} 


\subsection{Other examples}

In this section, we look at other examples of categories of fractional Hecke eigensheaves. First, we consider the abelian case, when $G=\mathbb{C}^{\times}$or an arbitrary torus. Then we consider the case of ${ }^{\mathrm{L}} G$-local systems (where $G$ is an arbitrary reductive group), whose group of automorphisms is the center of ${ }^{\mathrm{L}} G$. Finally, we discuss the geometric Eisenstein series.

6.6.1. The case of $\mathbb{C}^{\times}$Let $G=\mathbb{C}^{\times}$, so that ${ }^{\mathrm{L}} G=\mathbb{C}^{\times}$as well. Let $\mathcal{E}$ be a $\mathbb{C}^{\times}$-local system on $C$, which we will view as a rank 1 local system. The objects of the category $\mathcal{A} u t_{\mathcal{E}}$ are the data $(\mathcal{F}, \alpha)$, where $\mathcal{F}$ is a $\mathcal{D}$-module on Pic, the Picard scheme of $C$ and

$$
\alpha: H_{1}(\mathcal{F}) \stackrel{\sim}{\longrightarrow} \mathcal{E} \otimes \mathcal{F}
$$

Here $H_{1}$ is the Hecke functor corresponding to the identity character $\mathbb{C}^{\times} \rightarrow \mathbb{C}^{\times}$

$$
H_{1}(\mathcal{F})=p^{*}(\mathcal{F})
$$

where

$$
p: C \times \mathrm{Pic} \rightarrow \mathrm{Pic}, \quad(x, \mathcal{L}) \mapsto \mathcal{L}(-x) .
$$

This Hecke property automatically implies the Hecke property for the Hecke functors $H_{n}, n \in \mathbb{Z}$, corresponding to other characters of $\mathbb{C}^{\times}$. The $\mathcal{D}$-module Fourier-Mukai equivalence, due to $[51,66]$, implies that $\mathcal{F}$ is a direct sum of copies of a particular flat line bundle on Pic. From now on, we will denote by $\mathcal{F}$ this flat line bundle.

Since Pic is the disjoint union of its components $\operatorname{Pic}_{n}, n \in \mathbb{Z}$, corresponding to line bundles of degree $n$, each $\mathcal{D}$-module $\mathcal{F}$ on Pic decomposes into a direct sum

$$
\mathcal{F}=\bigoplus_{n \in \mathbb{Z}} \mathcal{F}_{n}
$$

The automorphism group of any rank one local system $\mathcal{E}$ is $\mathbb{C}^{\times}$. Hence we have an action of $\mathbb{C}^{\times}$on the category $\mathcal{A} u t_{\mathcal{E}}$. It is given by the formula

$$
\lambda \cdot(\mathcal{F}, \alpha) \mapsto(\mathcal{F}, \lambda \alpha), \lambda \in \mathbb{C}^{\times} .
$$

Now let us describe the corresponding category of fractional Hecke eigensheaves. Let $\mathcal{C}$ be the category whose objects are the direct sums of the $\mathcal{D}$-modules $\mathcal{F}_{n}, n \in \mathbb{Z}$, appearing in the decomposition (6.13) of the Hecke 
eigensheaf $\mathcal{F}$. These are flat vector bundles on the components $\mathrm{Pic}_{n} \subset \mathrm{Pic}$. The action of the category $\operatorname{Rep}\left(\mathbb{C}^{\times}\right)$on $\mathcal{C}$ is given by the formula

$$
[m] \star \mathcal{F}_{n}=\mathcal{F}_{n+m}
$$

where $[m]$ denotes the one-dimensional representation corresponding to the character $\mathbb{C}^{\times} \rightarrow \mathbb{C}^{\times}, a \mapsto a^{m}$.

An object of the category $\mathcal{A} u t_{\mathcal{E}}^{\prime}$ of fractional Hecke eigensheaves is then given by the data $(\mathcal{K}, \alpha)$, where $\mathcal{K} \in \mathcal{C}$ and

$$
\alpha: H_{1}(\mathcal{K}) \stackrel{\sim}{\longrightarrow} \mathcal{E} \otimes([1] \star \mathcal{K}) .
$$

The isomorphism $\alpha$ gives rise to a unique isomorphism

$$
\alpha_{n}: H_{n}(\mathcal{K}) \stackrel{\sim}{\longrightarrow} \mathcal{E}^{\otimes n} \otimes([n] \star \mathcal{K}), \quad n \in \mathbb{Z},
$$

satisfying the required properties.

Note that the regular Hecke property of $\mathcal{F}$ given by formula (6.12) is equivalent to the fractional Hecke property for $\mathcal{F}_{n}, n \in \mathbb{Z}$. Therefore, we obtain an isomorphism (6.14) for $\mathcal{K}=\mathcal{F}_{m}$ and hence for all objects of $\mathcal{C}$.

This example of a category of fractional Hecke eigensheaves should be contrasted with our main example for $G=S L_{2}$ coming from the endoscopy. In the latter case, the group of automorphisms is a finite group $\mathbb{Z}_{2}$. Hence we have a finite decomposition of a regular Hecke eigensheaf, $\mathcal{F}=\mathcal{F}_{+} \oplus \mathcal{F}_{-}$, with the summands that are labeled, albeit noncanonically, by irreducible representations of $\mathbb{Z}_{2}$. In the other example, the group of automorphisms is the Lie group $\mathbb{C}^{\times}$. Hence a regular Hecke eigensheaf decomposes into a direct sum (6.13) of infinitely many summands that are labeled, this time canonically, by irreducible representations of $\mathbb{C}^{\times}$.

An important difference is that in the endoscopic example there is no canonical equivalence between the category $\mathcal{C}$ of fractional Hecke eigensheaves and the category $\operatorname{Rep}\left(\mathbb{Z}_{2}\right)$ (in other words, we cannot distinguish between $\mathcal{F}_{+}$and $\mathcal{F}_{-}$), whereas in the other example, we identify it canonically with $\operatorname{Rep}\left(\mathbb{C}^{\times}\right)$(indeed, there is a canonical object $\mathcal{F}_{0}$ supported on the component $\mathrm{Pic}_{0}$ of line bundles of degree 0 ). The reason for this will be discussed in Section 10.

6.6.2. Arbitrary torus This example of category of fractional Hecke eigensheaves generalizes in a straightforward way to the case when $G$ is an arbitrary torus. In this case, the corresponding moduli space $\mathrm{Bun}_{T}$ decomposes into a disjoint union of components $\operatorname{Bun}_{T, \chi}$, where $\chi$ runs over the 
lattice $\check{P}$ of characters of ${ }^{\mathrm{L}} T$. The regular Hecke eigensheaf $\mathcal{F}$ therefore decomposes into a direct sum

$$
\mathcal{F}=\bigoplus_{\chi \in \check{P}} \mathcal{F}_{\chi}
$$

where $\mathcal{F}_{\chi}$ is supported on $\operatorname{Bun}_{T, \chi}$. The $\mathcal{D}$-modules $\mathcal{F}_{\chi}$ are then the building blocks of the category of fractional Hecke eigensheaves. They satisfy the fractional Hecke property

$$
H_{\lambda}\left(\mathcal{F}_{\chi}\right) \simeq \mathcal{F}_{\lambda+\chi}=[\lambda] \star \mathcal{F}_{\chi}
$$

where $H_{\lambda}$ is the Hecke functor corresponding to a character $\lambda \in \check{P}$. The objects of the category $\mathcal{A} u t_{\mathcal{E}}^{\prime}$ are collections $\left(\mathcal{K},\left(\alpha_{\lambda}\right)_{\lambda \in \check{P}}\right)$, where $\mathcal{K}$ is a direct sum of copies of $\mathcal{F}_{\chi}$ with some multiplicities and $\alpha_{\lambda}$ form a system of compatible isomorphisms (6.16).

6.6.3. The center as the automorphism group This has an analog for an arbitrary reductive group $G$. Namely, let ${ }^{\mathrm{L}} Z$ be the center of the dual group ${ }^{\mathrm{L}} G$. Suppose that $\mathcal{E}$ is an ${ }^{\mathrm{L}} G$-local system on $C$ whose group of automorphisms is precisely ${ }^{\mathrm{L}} Z$. A generic local system has this property. In this case, we expect that there is a unique regular Hecke eigensheaf $\mathcal{F}$ with the eigenvalue $\mathcal{E}$ which is irreducible on each connected component of $\operatorname{Bun}_{G}$ (and any other Hecke eigensheaf is a direct sum of copies of $\mathcal{F}$ ). The connected components of $\operatorname{Bun}_{G}$ are labeled precisely by the lattice $\check{P}$ of characters of ${ }^{\mathrm{L}} Z$, so we are in the same situation as above: the sheaf $\mathcal{F}$ decomposes

$$
\mathcal{F}=\bigoplus_{\chi \in \check{P}} \mathcal{F}_{\chi},
$$

where $\mathcal{F}_{\chi}$ is supported on the component corresponding to $\chi$. The $\mathcal{D}$-modules $\mathcal{F}_{\chi}$ are then the building blocks of the category of fractional Hecke eigensheaves, satisfying property (6.16). The objects of the category $\mathcal{A} u t_{\mathcal{E}}^{\prime}$ are then defined as in the case when $G$ is a torus. We expect that this category is equivalent to the category $\operatorname{Rep}\left({ }^{\mathrm{L}} Z\right)$. This matches the structure of the category of $B$-branes at the point of the moduli stack of ${ }^{\mathrm{L}} G$-local systems corresponding to $\mathcal{E}$, which is also equivalent to $\operatorname{Rep}\left({ }^{\mathrm{L}} Z\right)$, because ${ }^{\mathrm{L}} Z$ is assumed to be the group of automorphisms of $\mathcal{E}$ (see Section 2.2).

6.6.4. Geometric Eisenstein series As our last example, we describe the category of fractional Hecke eigensheaves for non-abelian groups using 
the construction of geometric Eisenstein series from $[12,50]$. More precisely, let $G$ be a reductive algebraic group and $T$ its maximal torus. Then ${ }^{\mathrm{L}} T$ is a maximal torus in ${ }^{\mathrm{L}} G$. Let $\mathcal{E}$ be an ${ }^{\mathrm{L}} T$-local system, which we view as an ${ }^{\mathrm{L}} G$-local system. Suppose that $\mathcal{E}$ is generic, in the sense that the rank 1 local systems corresponding to the roots of ${ }^{\mathrm{L}} G$ are all nontrivial. Then the group of automorphisms of $\mathcal{E}$ is equal to ${ }^{\mathrm{L}} T$. In this case, there is a geometric construction of a Hecke eigensheaf $\mathcal{F}_{G}$ on $\operatorname{Bun}_{G}$ with the eigenvalue $\mathcal{E}[12,50]$, starting from a Hecke eigensheaf $\mathcal{F}_{T}$ with respect to $\mathcal{E}$, considered as an ${ }^{\mathrm{L}} T$-local system.

By construction, decomposition (6.15) gives rise to a decomposition of $\mathcal{F}_{G}$,

$$
\mathcal{F}_{G}=\bigoplus_{\chi \in \check{P}} \mathcal{F}_{G, \chi}
$$

The $\mathcal{D}$-modules $\mathcal{F}_{G, \chi}$ are then the building blocks of the category of fractional Hecke eigensheaves corresponding to $\mathcal{E}$. We define the category $\mathcal{C}$ as the category whose objects are direct sums of the $\mathcal{D}$-modules $\mathcal{F}_{G, \chi}, \chi \in \check{P}$. The category $\operatorname{Rep}\left({ }^{\mathrm{L}} T\right)$ acts on it by the formula

$$
[\lambda] \star \mathcal{F}_{G, \chi}=\mathcal{F}_{G, \chi+\lambda}
$$

The objects of the corresponding category $\mathcal{A} u t_{\mathcal{E}}^{\prime}$ are collections $\left(\mathcal{K},\left(\alpha_{\lambda}\right)_{\lambda \in \check{P}}\right)$, where $\mathcal{K} \in \mathcal{C}$ and the $\alpha_{\lambda}$ 's form a system of compatible isomorphisms (see formula $(6.10))$

$$
\alpha_{\lambda}: H_{V}\left(\mathcal{F}_{G, \chi}\right) \stackrel{\sim}{\longrightarrow} \bigoplus_{\mu} V(\mu) \otimes\left([\lambda] \star \mathcal{F}_{G, \chi}\right)=\bigoplus_{\mu} V(\mu) \otimes \mathcal{F}_{G, \chi+\mu}
$$

Here $V$ is a representation of ${ }^{\mathrm{L}} G$, and $V(\mu)$ is the subspace of $V$ of weight $\mu$, so that we have

$$
\operatorname{Res}_{T}(V)=\bigoplus_{\mu} V(\mu)
$$

Note that in the abelian case when $G=T$, decomposition (6.15) has geometric origin: it corresponds to the splitting of $\mathrm{Bun}_{T}$ into a union of connected components. However, for non-abelian $G$, decomposition (6.17) of the corresponding Eisenstein sheaves is not directly linked in any obvious way to the geometry of the underlying moduli stack of $G$-bundles. This is similar to what happens in the endoscopic case.

It would be interesting to construct explicitly the $A$-branes corresponding to the Eisenstein sheaves $\mathcal{F}_{G}$ and $\mathcal{F}_{G, \chi}$ using the mirror symmetry of 
the Hitchin fibrations, by analogy with the endoscopic example. On the $B$-model side, this corresponds to a more complicated singularity, with a continuous group of automorphisms (the maximal torus of ${ }^{\mathrm{L}} G$ ) rather than a finite group, as in the endoscopic examples (this is reflected in particular in the fact that the Hitchin fibers now have infinitely many irreducible components). Because of that, the analysis of the mirror symmetry becomes more subtle in this case. We plan to discuss this in more detail elsewhere.

\section{The classical story}

In this section, we recall the setup of endoscopy and $L$-packets in the classical theory of automorphic forms, discovered by Labesse and Langlands [42]. We will discuss potential implications of the geometric picture outlined above for the classical theory in the next section.

\subsection{Local and global Langlands correspondence}

Let us first recall the general setup of the classical and the geometric Langlands correspondence. For simplicity, we will restrict ourselves here to the unramified situation.

The geometric Langlands correspondence predicts, in the first approximation, that for each irreducible ${ }^{\mathrm{L}} G$-local system $\mathcal{E}$ on $C$ there exists a unique (up to an isomorphism) Hecke eigensheaf $\left(\mathcal{F}_{\mathcal{E}},\left(\alpha_{V}\right)\right)$ on $\operatorname{Bun}_{G}$ with eigenvalue $\mathcal{E}$, a notion discussed in detail in Section 6.3.

We wish to recall the relation between the geometric Langlands correspondence and the classical Langlands correspondence, in the case when the curve $C$ is defined over a finite field $k=\mathbb{F}_{q}$. So let $C$ be such a curve and $F$ the field of rational functions of $C$. For example, if $C=\mathbb{P}^{1}$, then $F$ consists of fractions $f(z) / g(z)$, where $f(z)$ and $g(z)$ are polynomials (in variable $z$ ) over $k$ which do not have common factors (and $g(z)=z^{m}+\cdots$ is monic). For each closed point $x$ of $C$, we have the local field $F_{x} \simeq k_{x}((t))$, where $t$ is a local coordinate at $x$, and its ring of integers $\mathcal{O}_{x} \simeq k_{x}[[t]]$. Here $k_{x}$ is the residue field of $x$, which is in general a finite extension of $k$, and hence is isomorphic to $\mathbb{F}_{q^{m}}$ for some $m \geq 1$. This number is called the degree of $x$ and is denoted by $\operatorname{deg}(x)$. For example, in the case when $C=\mathbb{P}^{1}$, closed points correspond to irreducible monic polynomials in $k[z]$, and in addition there is the point $\infty$. The points with residue field $k$ correspond to polynomials of degree one, $z-a, a \in k$. A general irreducible monic polynomial $P(z)$ of degree $n$ corresponds to a closed point of $C$ of degree $n$. The field $k_{x}$ is just the quotient of $k[z]$ by the principal ideal generated by $P(z)$. 
The ring $\mathbb{A}_{F}$ of adéles of $F$ is by definition the restricted product

$$
\mathbb{A}_{F}=\prod_{x \in C}{ }^{\prime} F_{x}
$$

where the word "restricted" (and the prime in the notation) refers to the fact that elements of $\mathbb{A}_{F}$ are collections $\left(f_{x}\right)_{x \in C}$, where $f_{x} \in \mathcal{O}_{x}$ for all but finitely many $x \in C$.

Let $\operatorname{Gal}(\bar{F} / F)$ be the Galois group of $F$, the group of automorphisms of the separable closure $\bar{F}$ of $F$ (obtained by adjoining to $F$ the roots of all separable polynomials with coefficients in $F$ ), which preserve $F$ pointwise. We have a natural homomorphism $\operatorname{Gal}(\bar{F} / F) \rightarrow \operatorname{Gal}(\bar{k} / k)$. The group $\operatorname{Gal}(\bar{k} / k)$ is topologically generated by the Frobenius automorphism $\mathrm{Fr}: y \mapsto y^{q}$, and is isomorphic to the pro-finite completion $\widehat{\mathbb{Z}}$ of the group of integers $\mathbb{Z}$. The preimage of $\mathbb{Z} \subset \widehat{\mathbb{Z}}$ in $\operatorname{Gal}(\bar{F} / F)$ is the Weil group $W_{F}$ of $F$.

The Weil group (or, more precisely, its unramified quotient) is the arithmetic analog of the fundamental group of $C$. Therefore, the arithmetic analogue of a ${ }^{\mathrm{L}} G$-local system on $C$ is a homomorphism ${ }^{37}$

$$
\sigma: W_{F} \rightarrow{ }^{\mathrm{L}} G
$$

The global Langlands conjecture predicts, roughly speaking, that to each $\sigma$ corresponds an automorphic representation $\pi(\sigma)$ of the group $G\left(\mathbb{A}_{F}\right)$. This means that it may be realized in a certain space of functions on the quotient $G(F) \backslash G\left(\mathbb{A}_{F}\right) .{ }^{38}$

It is useful to relate this global Langlands conjecture to the local ones. Recall that for each closed point $x$ of $C$ (we will write $x \in C$ ) we have the local field $F_{x} \simeq k_{x}((t))$, which is a completion of $F$. We define its Weil group $W_{F_{x}}$ in the same way as above, as the preimage of $\mathbb{Z} \subset \widehat{\mathbb{Z}}=\operatorname{Gal}\left(\bar{k}_{x} / k_{x}\right)$ in $\operatorname{Gal}\left(\bar{F}_{x} / F_{x}\right)$ under the homomorphism $\operatorname{Gal}\left(\bar{F}_{x} / F_{x}\right) \rightarrow \operatorname{Gal}\left(\bar{k}_{x} / k_{x}\right)$. The group $W_{F_{x}}$ may be realized as a subgroup of the global Weil group $W_{F}$, but noncanonically. However, its conjugacy class in $W_{F}$ is canonical. Hence the equivalence class of $\sigma: W_{F} \rightarrow{ }^{\mathrm{L}} G$ as above gives rise to an equivalence class

\footnotetext{
${ }^{37}$ Here we need to consider ${ }^{\mathrm{L}} G$ over the field $\overline{\mathbb{Q}}_{\ell}$, where $\ell$ does not divide $q$, and the so-called $\ell$-adic homomorphisms (see, e.g., [25, Section 2.2]).

${ }^{38}$ This requires some explanation if $\pi(\sigma)$ does not appear in the "discrete spectrum," but we will ignore this technical issue here.
} 
of homomorphisms

$$
\sigma_{x}: W_{F_{x}} \rightarrow{ }^{\mathrm{L}} G \text {. }
$$

The local Langlands conjecture predicts, roughly speaking, that to each $\sigma_{x}$ we can associate a (smooth) irreducible representation $\pi_{x}$ of $G\left(F_{x}\right)$. Taking their restricted tensor product (see below), we obtain an irreducible representation

$$
\pi=\pi(\sigma)=\bigotimes_{x \in C}^{\prime} \pi_{x}
$$

of the adèlic group $G\left(\mathbb{A}_{F}\right)$. The compatibility between the global and local Langlands conjectures is the statement that this $\pi$ is automorphic. $\pi$ is then the automorphic representation corresponding to the global homomorphism $\sigma$. Schematically,

$$
\begin{aligned}
& \sigma_{x} \stackrel{\text { local }}{\longrightarrow} \pi_{x} \\
& \sigma \stackrel{\text { global }}{\longrightarrow} \pi=\bigotimes_{x \in C}^{\prime} \pi_{x} .
\end{aligned}
$$

Here the homomorphism $\sigma$ is assumed to be unramified at all but finitely many points of $C$. This means for all but finitely many $x \in C$ the homomorphism $\sigma_{x}: W_{F_{x}} \rightarrow{ }^{\mathrm{L}} G$ factors through the quotient $W_{F_{x}} \rightarrow \mathbb{Z}$. The generator of this quotient $\mathbb{Z} \subset \operatorname{Gal}\left(\bar{k}_{x} / k_{x}\right)$ is called the Frobenius element associated to $x$ and is denoted by $\mathrm{Fr}_{x}$. Since $\sigma_{x}$ is only well defined up to conjugation, we obtain that $\sigma_{x}\left(\mathrm{Fr}_{x}\right)$ gives rise to a well-defined conjugacy class in ${ }^{\mathrm{L}} G$. It is believed that the conjugacy classes obtained this way are always semisimple. Thus, we obtain a semi-simple conjugacy class $\sigma_{x}\left(\mathrm{Fr}_{x}\right)$ in ${ }^{\mathrm{L}} G$ for all but finitely many $x \in C$.

The irreducible representation $\pi_{x}$ corresponding to an unramified $\sigma_{x}$ is also unramified, which means that its subspace of invariant vectors $\left(\pi_{x}\right)^{G\left(\mathcal{O}_{x}\right)} \subset \pi_{x}$ under the maximal compact subgroup $G\left(\mathcal{O}_{x}\right) \subset G\left(F_{x}\right)$ is one-dimensional. We will fix, once and for all, a nonzero $G\left(\mathcal{O}_{x}\right)$-invariant vector $v_{x} \in \pi_{x}$ for all such $x \in C$. Then the prime in formula (7.1) (indicating the "restricted tensor product") means that this space is spanned by vectors of the form $\bigotimes_{x \in C} w_{x}$, where for all but finitely many points $x$ we have $w_{x}=v_{x}$.

Since the vector $v_{x}$ is $G\left(\mathcal{O}_{x}\right)$-invariant, it is an eigenvector of the spherical Hecke algebra, defined as the algebra of compactly supported $G\left(\mathcal{O}_{x}\right)$ biinvariant functions on $G\left(F_{x}\right)$. By the Satake correspondence, this algebra is isomorphic to the representation $\operatorname{ring} \operatorname{Rep}\left({ }^{\mathrm{L}} G\right)$ (see, e.g., [25, Section 5.2]). 
Therefore, to each $V \in \operatorname{Rep}\left({ }^{\mathrm{L}} G\right)$ corresponds an element of the spherical Hecke algebra, which we denote by $T_{V, x}$. These operators, which are function theoretic analog of the Hecke functors $H_{V, x}$ discussed in Section 6.3, act on $\pi_{x}$. The vector $v_{x} \in \pi_{x}$ is a joint eigenvector with respect to this action, and the eigenvalues are recorded by the conjugacy class of $\sigma_{x}\left(\operatorname{Fr}_{x}\right)$. Namely, we have ${ }^{39}$

$$
T_{V, x} \cdot v_{x}=\operatorname{Tr}\left(\sigma\left(\operatorname{Fr}_{x}\right), V\right) v_{x}
$$

Actually, this property determines $\pi_{x}$ uniquely.

\section{2. $L$-packets}

The picture of the local and global Langlands correspondences outlined above is correct (and has been proved) for $G=G L_{n}$. But for other groups, one needs to make some adjustments. The most important adjustment is that in general the local Langlands correspondence assigns to each $\sigma_{x}$ not a single equivalence class of irreducible representations $\pi_{x}$, but a collection $\left\{\pi_{x, \alpha}\right\}_{\alpha \in A_{x}}$, called a (local) L-packet. Usually, this happens for infinitely many points $x \in C$, and so picking a particular representation in each of these $L$-packets, we obtain infinitely many representations of $G\left(\mathbb{A}_{F}\right)$. It turns out that in general not all of them are automorphic, and those which are automorphic may occur in the space of functions on $G(F) \backslash G\left(\mathbb{A}_{F}\right)$ with different multiplicities. There is a beautiful combinatorial formula for this multiplicity for those homomorphisms $\sigma: W_{F} \rightarrow{ }^{\mathrm{L}} G$ which factor through the groups ${ }^{\mathrm{L}} H$ dual to the endoscopic group of $G[40,42]$.

This phenomenon was first discovered in the case of $G=S L_{2}$ by Labesse and Langlands in [42]. Let us briefly summarize their results in the unramified case.

In this case ${ }^{\mathrm{L}} G=S O_{3}=P G L_{2}$. Let $E$ be an unramified quadratic extension of $F$. This is the field of functions $k\left(C^{\prime}\right)$ of a degree 2 unramified covering $C^{\prime}$ of our curve $C$. Let $\mu$ be a character, that is, a one-dimensional ( $\ell$-adic) representation of the Weil group $W_{E}$. The group $W_{F}$ contains $W_{E}$ as a normal subgroup, and the quotient is isomorphic to $\operatorname{Gal}(E / F)=\{1, \tau\}$. Let

$$
\sigma: W_{F} \rightarrow P G L_{2}
$$

\footnotetext{
${ }^{39}$ Here and below, we skip factors of the form $q_{x}^{m}$ on the right-hand sides of this and other similar formulas.
} 
be the projectivization of the two-dimensional representation $\tilde{\sigma}$ of $W_{F}$ induced from $\mu$,

$$
\widetilde{\sigma}=\operatorname{Ind}_{W_{E}}^{W_{F}} \mu .
$$

It is clear that the image of $\sigma$ belongs to the subgroup $O_{2} \subset S O_{3}=P G L_{2}$. We will assume in what follows that the image does not belong to the subgroup $\mathbb{Z}_{2} \times \mathbb{Z}_{2} \subset \mathrm{O}_{2}$ or to the connected component $\mathrm{SO}_{2}$ of $\mathrm{O}_{2}$. In this case, the centralizer of the image of $\sigma$ is equal to $\mathbb{Z}_{2}$. The corresponding homomorphisms $\sigma: W_{F} \rightarrow P G L_{2}$ are the arithmetic analogues of the $S_{3}=P G L_{2}$-local systems that we have considered above in the geometric setting.

Let us now look at the local homomorphisms

$$
\sigma_{x}: W_{F_{x}} \rightarrow P G L_{2}
$$

corresponding to $\sigma$. There are two possibilities:

- The point $x$ is split in $E / F$. This means that the preimage of $x$ in $C^{\prime}$ consists of two closed points, which we will denote by $y_{1}(x)$ and $y_{2}(x)$, and their residue fields are both equal to the residue field of $x$. In this case, $W_{F_{x}}$ is isomorphic to both $W_{E_{y_{1}(x)}}$ and $W_{E_{y_{2}(x)}}$, which, when realized as subgroups of $W_{F}$, are conjugate to each other:

$$
\widetilde{\tau} W_{E_{y_{1}(x)}} \widetilde{\tau}^{-1}=W_{E_{y_{2}(x)}}
$$

where $\widetilde{\tau}$ projects onto the nontrivial element $\tau \in \operatorname{Gal}(E / F)$ under the homomorphism $W_{F} \rightarrow \operatorname{Gal}(E / F)$.

- The point $x$ is not split. Then the preimage of $x$ in $C^{\prime}$ consists of one closed point, which we will denote by $y(x)$, and its residue field $k_{y(x)}$ is a quadratic extension of the residue field $k_{x}$ of $x$. Thus, if $k_{x} \simeq$ $\mathbb{F}_{q^{n}}$, then $k_{y(x)} \simeq \mathbb{F}_{q^{2 n}}$. In this case, $W_{F_{x}}$ contains $W_{E_{y(x)}}$ as a normal subgroup, and the quotient is isomorphic to $\operatorname{Gal}\left(E_{y(x)} / F_{x}\right) \simeq \mathbb{Z}_{2}$.

In the first case, $\sigma_{x}$ is equivalent to the projectivization of the twodimensional representation $\widetilde{\sigma}_{x}$ of $W_{F_{x}}$ defined by the formula (here we identify $W_{F_{x}}$ with $\left.W_{E_{y_{1}(x)}}\right)$ :

$$
g \mapsto\left(\begin{array}{cc}
\mu_{y_{1}(x)}(g) & 0 \\
0 & \mu_{y_{2}(x)}\left(\widetilde{\tau} g \widetilde{\tau}^{-1}\right)
\end{array}\right), g \in W_{E_{y_{1}(x)}}
$$

where $\mu_{y_{i}(x)}$ denotes the restriction of $\mu$ to $W_{E_{y_{i}(x)}}$. 
In the second case, $\sigma_{x}$ is isomorphic to the projectivization of the twodimensional induced representation

$$
\widetilde{\sigma}_{x}=\operatorname{Ind}_{W_{E_{y(x)}}^{W_{F_{x}}}}^{\mu_{y}(x)} \cdot
$$

As shown by Labesse-Langlands, the local $L$-packet contains either one or two irreducible representations of $S L_{2}\left(F_{x}\right)$. In both cases, they appear as irreducible summands of a single irreducible representation of $G L_{2}\left(F_{x}\right)$.

From now on, we will focus on the unramified homomorphisms from the Weil group $W_{F}$ to $P G L_{2}$. We have already assumed that the covering $C^{\prime} \rightarrow C$ is unramified, and from now on we will assume that the character $\mu$ of $W_{E}$ is unramified as well. Then our $\sigma$ is unramified, and hence so are the local homomorphisms $\sigma_{x}: W_{F_{x}} \rightarrow P G L_{2} \cdot{ }^{40}$ Hence $\sigma_{x}$ is determined by the image $\sigma_{x}\left(\mathrm{Fr}_{x}\right)$ in $P G L_{2}$ of the Frobenius conjugacy class $\mathrm{Fr}_{x}$. There are two possibilities: if $\sigma_{x}\left(\mathrm{Fr}_{x}\right)$ is the conjugacy class of the element

$$
\left(\begin{array}{ll}
0 & 1 \\
1 & 0
\end{array}\right) \in P G L_{2}
$$

whose centralizer has two connected components, then the $L$-packet consists of two irreducible representations of $S L_{2}\left(F_{x}\right)$; otherwise, the centralizer is connected, and the $L$-packet consists of a single irreducible representation.

The former scenario may occur in two ways. First, suppose that the point $x$ is nonsplit. Choose the basis $\left\{1, \mathrm{Fr}_{x}\right\}$ in the induced representation (7.4). Then we have

$$
\widetilde{\sigma}_{x}\left(\operatorname{Fr}_{x}\right)=\left(\begin{array}{cc}
0 & \mu\left(\operatorname{Fr}_{y(x)}\right) \\
1 & 0
\end{array}\right) .
$$

Therefore, we obtain that $\sigma_{x}\left(\mathrm{Fr}_{x}\right)$ is the conjugacy class of (7.5). The second possibility is that $x$ is split, and the values of $\mu$ on $\operatorname{Fr}_{y_{1}(x)}$ and $\operatorname{Fr}_{y_{2}(x)}$ differ by the minus sign. In this case $\widetilde{\sigma}_{x}\left(\mathrm{Fr}_{x}\right)$ is conjugate to

$$
\mu\left(\operatorname{Fr}_{y_{i}(x)}\right)\left(\begin{array}{rr}
1 & 0 \\
0 & -1
\end{array}\right) \sim\left(\begin{array}{rr}
1 & 0 \\
0 & -1
\end{array}\right) \in P G L_{2}, \quad i=1,2
$$

Therefore, $\sigma_{x}\left(\mathrm{Fr}_{x}\right)$ is again the conjugacy class of (7.5) in $P G L_{2}$. However, we will see below that the second case does not play an important role in the endoscopy.

\footnotetext{
${ }^{40}$ Note, however, that there are ramified characters $\mu$ such that the corresponding homomorphism $\sigma$ is unramified. Imposing the condition that $\mu$ be unramified is similar to considering $P G L_{2}$-local systems with $w_{2}=0$ in the geometric theory.
} 
In both of these cases, the corresponding $L$-packet consists of two irreducible representations, $\pi_{x}^{\prime}$ and $\pi_{x}^{\prime \prime}$, of $S L_{2}\left(F_{x}\right)$. We will discuss them in more detail in the next section.

Thus, we see that at infinitely many points of $C$ we have a binary choice between the two possible irreducible representations $\pi_{x}$ of $S L_{2}\left(F_{x}\right)$ in the tensor product (7.1). However, it turns out that only about one half of those choices, at the nonsplit points of $C$ (with respect to the covering $C^{\prime} \rightarrow C$ ), gives rise to automorphic representations of $S L_{2}\left(\mathbb{A}_{F}\right)$. More precisely, if we choose particular representations $\pi_{x}$ in the local $L$-packets at all but one nonsplit point of $C$, say $y$, then only one of the two representations $\left\{\pi_{y}^{\prime}, \pi_{y}^{\prime \prime}\right\}$ of the local $L$-packet at the remaining point $y$ will complete the tensor product $\bigotimes_{x \neq y}^{\prime} \pi_{x}$ to an automorphic representation $\pi$ of $S L_{2}\left(\mathbb{A}_{F}\right)$.

The precise description of the collections of irreducible representations whose tensor products are automorphic will be presented in the next section. This description was first given by Labesse and Langlands [42] using the trace formula. In Appendix, we will give an alternative derivation of this description using an explicit formula for the Hecke eigenfunctions due to Weil [77] and Jacquet-Langlands [37] using the Fourier transform of the Whittaker functions.

\subsection{Spaces of invariant vectors}

Our goal in what follows is to pass from the classical to the geometric setting. The first step in this direction is to cut down the infinite-dimensional representation $\pi=\bigotimes_{x \in C}^{\prime} \pi_{x}$ of $G\left(\mathbb{A}_{F}\right)$ to a finite-dimensional vector space $\pi^{K}$ of $K$-fixed vectors, where $K$ is a compact subgroup of $G\left(\mathbb{A}_{F}\right)$. We take $K$ to be the product

$$
K=\prod_{x \in C} K_{x}
$$

of compact subgroups $K_{x} \subset G\left(F_{x}\right) \simeq G((t))$. A typical example is the subgroup $G\left(\mathcal{O}_{x}\right)=G[[t]]$. As we discussed in Section 7.1, any vector in $\pi$ is invariant under the subgroup that is the product of $G\left(\mathcal{O}_{x}\right)$ for all but finitely many $x$. If $\pi$ is automorphic, then $\pi^{K}$ is realized in the space of functions on the double quotient $G(F) \backslash G\left(\mathbb{A}_{F}\right) / K$, which are Hecke eigenfunctions for all $x \in C$ for which $K_{x}=G\left(\mathcal{O}_{x}\right)$. This double quotient has a geometric interpretation as the set of $\mathbb{F}_{q}$-points of a moduli stack of $G$-bundles on $C$ with parabolic (or level) structures at those points of $C$ where $K_{x}$ is not maximal compact (e.g., choosing $K_{x}$ to be the Iwahori subgroup corresponds to fixing a Borel reduction in the fibers of the $G$-bundles at $x$, etc.), and this fact will 
be used below in order to relate the classical and the geometric Langlands conjectures.

In the unramified case, the existence of nontrivial $L$-packets is related to the fact that there are inequivalent choices for a maximal compact subgroup of $G\left(F_{x}\right)$. For example, for $G=S L_{2}$, there are two inequivalent choices: one of them is $S L_{2}[[t]]$, and the other one is

$$
\left(\begin{array}{ll}
t & 0 \\
0 & 1
\end{array}\right) S L_{2}[[t]]\left(\begin{array}{cc}
t^{-1} & 0 \\
0 & 1
\end{array}\right)
$$

(they are conjugate in $G L_{2}((t))$, but not in $S L_{2}((t))$ ). We will denote these two subgroups by $K^{\prime}$ and $K^{\prime \prime}$, respectively. Naively, it looks like $K^{\prime}$ is a preferred choice, but that is because we have tacitly chosen as our initial datum the "constant" group scheme $S L_{2}$ over the curve $C$. However, our initial datum should really be the group $S L_{2}$ over the field $F$ of rational functions on $C$, or, in other words, a group scheme over the generic point of $C$. There are many ways to extend it to a group scheme over the entire $C$, and we have the freedom of extending it in such a way that at $x \in C$ we get as the group of sections over the disc $D_{x}$, a subgroup of $S L_{2}\left(F_{x}\right)=S L_{2}((t))$ that is conjugate to either $K^{\prime}$ or $K^{\prime \prime} .{ }^{41}$ This makes it clear that there is in a priori no canonical choice between the subgroups $K^{\prime}$ and $K^{\prime \prime}$.

However, in what follows, we will fix a particular extension of the group scheme $S L_{2}$ from the generic point of $C$ to the entire $C$; namely, the "constant" group scheme $C \times S L_{2}$. Thus, we will have a preferred compact subgroup $K_{x}^{\prime}=S L_{2}[[t]]$ at each point $x \in C$.

This ambiguity in the choice of a maximal compact subgroup of $S L_{2}\left(F_{x}\right)$ is closely related to the structure of the unramified local $L$-packets $\left\{\pi_{x}^{\prime}, \pi_{x}^{\prime \prime}\right\}$. Namely, choosing appropriate notation, the spaces of invariant vectors $\left(\pi_{x}^{\prime}\right)^{K_{x}^{\prime}}$ and $\left(\pi_{x}^{\prime \prime}\right)^{K_{x}^{\prime \prime}}$ are one-dimensional, whereas $\left(\pi_{x}^{\prime}\right)^{K_{x}^{\prime \prime}}=\left(\pi_{x}^{\prime \prime}\right)^{K_{x}^{\prime}}=0$.

Let us return to the setting of the previous section. Thus, we are given an unramified degree 2 covering $C^{\prime}$ of $C$ and an unramified character $\mu$ of $W_{E}$, where $E$ is the field of functions on $C^{\prime}$. We let $\sigma$ be a homomorphism $W_{F} \rightarrow P G L_{2}$ defined as above. Now let us fix one of the two maximal compact subgroups $K_{x}$ at each point of $x$ at which $\sigma_{x}\left(\operatorname{Fr}_{x}\right)$ is conjugate to (7.5). We will assume that $K_{x}=K_{x}^{\prime}=S L_{2}[[t]]$ at all but finitely many points.

Thus, we have an irreducible representation $\pi_{x}$ of $S L_{2}\left(F_{x}\right)$ such that $\left(\pi_{x}\right)^{K_{x}}$ is one-dimensional at all of those points. Denote by $S$ the finite set

\footnotetext{
${ }^{41}$ As an analogy, consider the datum of a line bundle over $C \backslash\left\{x_{1}, \ldots, x_{m}\right\}$ - it
} can be extended to a line bundle on the entire curve $C$ in many different ways. 
of the nonsplit points $x \in C$ (with respect to the covering $C^{\prime} \rightarrow C$ ) such that $K_{x}=K_{x}^{\prime \prime}$. Let $\# S$ be its cardinality. The following statement is due to [42] (for an alternative proof, see Appendix).

Theorem 7.1. The representation

$$
\bigotimes_{x \in C}^{\prime} \pi_{x}
$$

of $S L_{2}\left(\mathbb{A}_{F}\right)$ is an automorphic representation if and only if $\# S$ is even.

Labesse and Langlands formulate this condition in the following neat form, which allows a generalization to other groups [40]. Denote by $S_{\sigma}$ the group of automorphisms of our homomorphism $\sigma: W_{F} \rightarrow P G L_{2}$, that is, the centralizer of the image of $\sigma$ in $P G L_{2} \cdot{ }^{42}$ Let $S_{\sigma}^{0}$ be its connected component. Likewise, for each $x \in C$, let $S_{\sigma_{x}}$ be the group of automorphisms of $\sigma_{x}: W_{F} \rightarrow P G L_{2}$ and $S_{\sigma_{x}}^{0}$ its connected component. We have natural homomorphisms $S_{\sigma} \rightarrow S_{\sigma_{x}}$ and $S_{\sigma}^{0} \rightarrow S_{\sigma_{x}}^{0}$, and hence a homomorphism

$$
\frac{S_{\sigma}}{S_{\sigma}^{0}} \rightarrow \frac{S_{\sigma_{x}}}{S_{\sigma_{x}}^{0}} .
$$

In our case, for generic $\sigma$ in the class that we are considering here we have $S_{\sigma}=S_{\sigma} / S_{\sigma}^{0}=\mathbb{Z}_{2}$, generated (with respect to the natural basis in the induced representation) by the element

$$
\left(\begin{array}{rr}
1 & 0 \\
0 & -1
\end{array}\right)
$$

of $P G L_{2}$ (this is the centralizer of $\mathrm{O}_{2} \subset P G L_{2}$ ).

Now consider the local groups. If $x$ is a split point of $C$ and the ratio of the eigenvalues of $\sigma_{x}\left(\mathrm{Fr}_{x}\right)$ is not equal to -1 , then $S_{\sigma_{x}}$ is a connected torus, and so $S_{\sigma_{x}} / S_{\sigma_{x}}^{0}$ is trivial. Next, consider the case of split points for which the ratio of the eigenvalues of $\sigma_{x}\left(\mathrm{Fr}_{x}\right)$ is equal to -1 . Then the group $S_{\sigma_{x}}$ is the subgroup $\mathrm{O}_{2}$ of $P G L_{2}$, which is the centralizer of (7.10) (see formula (7.6)), and so $S_{\sigma} / S_{\sigma}^{0}=\mathbb{Z}_{2}$. But the global automorphism (7.10) lands in the connected component $S_{\sigma_{x}}^{0}$ of $S_{\sigma_{x}}$, so the homomorphism (7.9) is trivial in this case. Finally, if $x$ is nonsplit, we find that $S_{\sigma_{x}}$ is the centralizer of (7.5), which is also isomorphic to $\mathrm{O}_{2}$. However, now the element (7.10)

${ }^{42}$ This is the arithmetic analog of the group $\Gamma$ discussed in the previous section. We denote it by $S_{\sigma}$ in order to follow the standard notation. 
lands in the other connected component, and so the homomorphism (7.9) is nontrivial in this case.

The idea of [42] is that irreducible representations of $S L_{2}\left(F_{x}\right)$ from the local $L$-packet should be labeled by irreducible representations of $S_{\sigma_{x}} / S_{\sigma_{x}}^{0}$. Thus, if this group is trivial, there is only one irreducible representation in the $L$-packet. If this group is isomorphic to $\mathbb{Z}_{2}$, then there are two. According to our conventions, the representation with nontrivial space of invariants of $K_{x}^{\prime}$ will correspond to the trivial representation of $\mathbb{Z}_{2}$, and the one with nontrivial invariants of $K_{x}^{\prime \prime}$ will correspond to the sign representation of $\mathbb{Z}_{2}$. Now the tensor product (7.8) gives rise to an irreducible representation of the group $\prod_{x \in C} S_{\sigma_{x}} / S_{\sigma_{x}}^{0}$, on which all but finitely many factors act trivially.

Theorem 7.1 may then be reformulated as saying that (7.8) is automorphic if and only if $S_{\sigma} / S_{\sigma}^{0}$ acts trivially on the corresponding representation of $\prod_{x \in C} S_{\sigma_{x}} / S_{\sigma_{x}}^{0}$, via the diagonal homomorphism

$$
\frac{S_{\sigma}}{S_{\sigma}^{0}} \rightarrow \prod_{x \in C} \frac{S_{\sigma_{x}}}{S_{\sigma_{x}}^{0}} .
$$

Note that, according to the above discussion, if $x$ is split, then the homomorphism (7.9) has trivial image, even if the group $S_{\sigma_{x}} / S_{\sigma_{x}}^{0}$ is nontrivial. Therefore, we may choose either of the two irreducible representations of $S L_{2}\left(F_{x}\right)$ from the local $L$-packet associated to such a point as $\pi_{x}$, and in both cases the corresponding representations (7.8) will simultaneously be automorphic or not. In this sense, the split points do not affect the automorphy of the representation (7.8), unlike the nonsplit points, for which it is crucial which one of the two members of the $L$-packet we choose as the local factor of (7.8).

Suppose now that \#S is even and so the representation (7.8) is automorphic. Then the one-dimensional vector space

$$
\bigotimes_{x \in C}\left(\pi_{x}\right)^{K_{x}}
$$

may be realized in the space of functions on

$$
S L_{2}(F) \backslash S L_{2}\left(\mathbb{A}_{F}\right) / \prod_{x \in C} K_{x} .
$$

Moreover, any nonzero vector in (7.11) gives rise to a Hecke eigenfunction $f$ on (7.12) with the eigenvalues prescribed by the conjugacy class $\sigma_{x}\left(\operatorname{Fr}_{x}\right)$. This means that it is an eigenfunction of the Hecke operator 
$T_{W, x}$ corresponding to the adjoint representation $W$ of $P G L_{2}$ and a point $x \in C$, that is,

$$
T_{W, x} \cdot f=\operatorname{Tr}\left(\sigma_{x}\left(\operatorname{Fr}_{x}\right), W\right) f
$$

where $\mathrm{Fr}_{x}$ is the Frobenius conjugacy class corresponding to $x$ in $W_{F}$. Here $T_{W, x}$ is a generator of the spherical Hecke algebra of $K_{x}$ bi-invariant compactly supported functions on $S L_{2}\left(F_{x}\right)$. For either choice of $K_{x}$ this algebra is canonically isomorphic to $\operatorname{Rep}\left(P G L_{2}\right)$, and under this isomorphism $T_{W, x}$ corresponds to the class of the adjoint representation of $P G L_{2}$ (see Section 8.1 below for more details on the Hecke property).

Finally, suppose that \#S is odd. Then the representation (7.8) is not automorphic. Hence the one-dimensional vector space (7.11) cannot possibly be realized in the space of functions on (7.12). In other words, any function on (7.12) satisfying (7.13) is necessarily identically equal to zero.

This should be contrasted with the generic situation, when the image of $\sigma: W_{F} \rightarrow P G L_{2}$ has trivial centralizer (recall that we are focusing here exclusively on the unramified homomorphisms $\sigma$ ). In this case, the group $S_{\sigma} / S_{\sigma}^{0}$ is trivial, so all representations (7.8), where the local factors $\pi_{x}$ are arbitrary representations from the local $L$-packets corresponding to $\sigma_{x}$, will be automorphic. If $\sigma_{x}\left(\mathrm{Fr}_{x}\right)$ is generic, then $S_{\sigma_{x}} / S_{\sigma_{x}}^{0}$ is trivial, and the corresponding $L$-packet contains one irreducible representation $\pi_{x}$ of $S L_{2}$ $\left(F_{x}\right)$. This $\pi_{x}$ has one-dimensional spaces of invariants under both $K_{x}^{\prime}$ and $K_{x}^{\prime \prime}$. It is also possible that for some $x \in C, \sigma_{x}\left(\mathrm{Fr}_{x}\right)$ is in the conjugacy class of (7.8). Then $S_{\sigma_{x}} / S_{\sigma_{x}}^{0}$ is equal to $\mathbb{Z}_{2}$, and the $L$-packet contains two irreducible representations, one of which has nonzero invariants with respect to $K_{x}^{\prime}$, and the other with respect to $K_{x}^{\prime \prime}$. However, inserting either of them as the local factor of $\pi$ at $x$, we will obtain an automorphic representation of $S L_{2}\left(\mathbb{A}_{F}\right)$.

Thus, we find that for a generic $\sigma$, given any choices of $K_{x}$ (that is, $K_{x}=K_{x}^{\prime}$ or $K_{x}=K_{x}^{\prime \prime}$ ), the corresponding space of Hecke eigenfunctions satisfying (7.12) on (7.12) is one-dimensional.

\subsection{The improper Hecke operators}

In the geometric theory, it was useful to consider, in addition to the "proper" Hecke operators corresponding to the three-dimensional adjoint representation of $\mathrm{SO}_{3}=P G L_{2}$, the "improper" ones corresponding to the twodimensional projective representation of this group. These operators also have counterparts in the classical theory. They are defined as follows. Given 
a point $x \in C$ and a coordinate $t$ at $x$, the operator $\widetilde{T}_{x}$ is the integral operator acting from functions on the double quotient (7.12) with $K_{x}=K_{x}^{\prime}=S L_{2}[[t]]$ to functions on the same double quotient, but with $K_{x}^{\prime}$ replaced by the subgroup $K_{x}^{\prime \prime}$ from Equation (7.7). It is given by the formula

$$
\left(\widetilde{T}_{x} \cdot f\right)(g)=\int_{M_{x}} f(g h) d h,
$$

where

$$
M_{x}=\left(\begin{array}{ll}
t & 0 \\
0 & 1
\end{array}\right) S L_{2}[[t]]\left(\begin{array}{ll}
t & 0 \\
0 & 1
\end{array}\right) .
$$

Now suppose that we have a Hecke eigenfunction $f^{\prime}$ (resp., $f^{\prime \prime}$ ) on (7.12) with $K_{x}=K_{x}^{\prime}$ (resp., $K_{x}=K_{x}^{\prime \prime}$ ), and with $K_{y}, y \neq x$, being the same, and satisfying the (proper) Hecke eigenfunction property (7.13). Each of these two functions is unique up to a scalar. Suppose that we can lift $\sigma$ to an unramified homomorphism $\tilde{\sigma}: W_{F} \rightarrow G L_{2}$. Then we can normalize the functions $f^{\prime}$ and $f^{\prime \prime}$ in such a way that both are equal to the restrictions to the appropriate double quotient (7.12) of a Hecke eigenfunction $\widetilde{f}$ for $G L_{2}\left(\mathbb{A}_{F}\right)$ corresponding to $\widetilde{\sigma}$ (see Appendix for more details). Since all Hecke operators for $G L_{2}$ commute with each other, we find that the function $\widetilde{T}_{x} \cdot f^{\prime}$ is a function on (7.12) with $K_{x}=K_{x}^{\prime \prime}$, which also satisfies (7.13). Moreover, we have

$$
\widetilde{T}_{x} \cdot f^{\prime}=\operatorname{Tr}\left(\widetilde{\sigma}_{x}\left(\operatorname{Fr}_{x}\right), V\right) f^{\prime \prime}
$$

where $V$ is the two-dimensional representation of $G L_{2}$. Thus, $\widetilde{T}_{x}$ is an intertwining operator between the two spaces of Hecke eigenfunctions for $S L_{2}\left(\mathbb{A}_{F}\right)$ if and only if the trace $\operatorname{Tr}\left(\widetilde{\sigma}_{x}\left(\mathrm{Fr}_{x}\right), V\right)$ is nonzero.

But this trace is equal to zero precisely when $\sigma_{x}\left(\mathrm{Fr}_{x}\right)$ is the conjugacy class of (7.5), and this is the special case when we have a nontrivial $L$-packet at $x$ ! In this case, $f^{\prime}$ and $f^{\prime \prime}$ correspond to two nonisomorphic representations of $S L_{2}\left(F_{x}\right)$ (one of which could be automorphic and the other one not). Formula (7.15) shows that in this case $\widetilde{T}_{x} \cdot f^{\prime}=0$. Thus, the improper Hecke operators give us another way to observe the nontriviality of the $L$-packets. They underscore the discrepancy between two spaces of Hecke eigenfunctions on the double quotients (7.12) corresponding to $K_{x}=K_{x}^{\prime}$ and $K_{x}=K_{x}^{\prime \prime}$ in the case of the endoscopic $\sigma$ : one of the two spaces could be one-dimensional and the other equal to zero. An analog of this phenomenon may be observed geometrically, as we will see in Section 8.7. 


\section{From Hecke eigensheaves to Hecke eigenfunctions}

We now wish to replace Hecke eigenfunctions by Hecke eigensheaves, geometric objects that allow us to link the classical Langlands correspondence to the geometric Langlands correspondence and ultimately to the mirror symmetry of the Hitchin fibrations for the dual groups discussed in the previous sections.

\subsection{Hecke eigensheaves in positive characteristic}

In our previous discussion of Hecke eigensheaves in Section 6.3, we had assumed that our curve was defined over $\mathbb{C}$. Then a Hecke eigensheaf corresponding to an ${ }^{\mathrm{L}} G$-local system $\mathcal{E}$ on $C$ is a $\mathcal{D}$-module $\mathcal{F}$ on $\operatorname{Bun}_{G}$ together with the additional data of isomorphisms $\alpha_{V}$ (see formula (6.5)). ${ }^{43}$ By using the Riemann-Hilbert correspondence, we may then switch from $\mathcal{D}$-modules to perverse sheaves (this is explained, e.g., in [25, Section 3.4]). Thus, Hecke eigensheaves may be viewed as objects of the category of perverse sheaves on $\operatorname{Bun}_{G}$, equipped with the isomorphisms (6.5).

Now we replace a complex curve by a curve $C$ defined over a finite field $k=\mathbb{F}_{q}$. The notion of perverse sheaves in characteristic 0 has an analog for algebraic varieties (or algebraic stacks) over a finite field (these are objects of the derived category of $\ell$-adic sheaves [7]). We have the moduli stack Bun $_{G}$ of $G$-bundles on our curve $C$ defined over $k$. This is an algebraic stack over $k$. Therefore, we have the notion of a Hecke eigensheaf on $\operatorname{Bun}_{G}$ corresponding to an unramified homomorphism $\sigma: W_{F} \rightarrow{ }^{\mathrm{L}} G$. Namely, we view $\sigma$ as an $\ell$-adic ${ }^{\mathrm{L}} G$-local system $\mathcal{E}$ on $C$. In other words, for each representation $V$ of ${ }^{\mathrm{L}} G$ the corresponding twist

$$
V_{\mathcal{E}}=\mathcal{E} \underset{\mathrm{L} G}{\times} V
$$

is a locally constant $\ell$-adic sheaf on $C$, and these sheaves are compatible with respect to the tensor product structure on representations of ${ }^{\mathrm{L}} G$. We also have Hecke functors $H_{V}, V \in \operatorname{Rep}\left({ }^{\mathrm{L}} G\right)$, defined in the same way as over $\mathbb{C}$.

A Hecke eigensheaf with "eigenvalue" $\mathcal{E}$ ( or $\sigma$ ) is, by definition, a perverse $\left(\ell\right.$-adic) sheaf $\mathcal{F}$ on $\operatorname{Bun}_{G}$ together with the additional data of isomorphisms (compare with (6.5))

$$
\alpha_{V}: H_{V}(\mathcal{F}) \stackrel{\sim}{\longrightarrow} V_{\mathcal{E}} \otimes \mathcal{F}
$$

\footnotetext{
${ }^{43}$ It is expected that this $\mathcal{D}$-module is holonomic and has regular singularities.
} 
These isomorphisms should be compatible with the tensor product structures and associativity on both sides. We will see below that to ensure the passage from Hecke eigensheaves to Hecke eigenfunctions we need to impose an additional equivariance condition.

To explain this passage in more detail, we recall that for any algebraic variety (or algebraic stack) $Y$ over $\mathbb{F}_{q}$, we may assign a function on the set of $\mathbb{F}_{q}$-points of $Y$ to any $\ell$-adic sheaf (or a complex) $\mathcal{F}$ on $Y[16,48]$. Indeed, let $y$ be an $\mathbb{F}_{q}$-point of $Y$ and $\bar{y}$ the $\overline{\mathbb{F}}_{q}$-point corresponding to an inclusion $\mathbb{F}_{q} \hookrightarrow \overline{\mathbb{F}}_{q}$. Then the pullback of $\mathcal{F}$ with respect to the composition $\bar{y} \rightarrow y \rightarrow Y$ is a ( $\ell$-adic) sheaf on a point Spec $\overline{\mathbb{F}}_{q}$. The data of such a sheaf is the same as the data of a $\overline{\mathbb{Q}}_{\ell}$-vector space, which we may think of as the stalk $\mathcal{F}_{\bar{y}}$ of $\mathcal{F}$ at $\bar{y}$. There is an additional piece of data on this vector space. Indeed, the Galois group $\operatorname{Gal}\left(\overline{\mathbb{F}}_{q} / \mathbb{F}_{q}\right)$ is the symmetry group of the morphism $\bar{y} \rightarrow y$, and therefore it acts on $\mathcal{F}_{\bar{y}}$. In particular, we have an action of the (geometric) Frobenius element $\mathrm{Fr}_{y}$, corresponding (the inverse of) the generator of the Galois group of $\mathbb{F}_{q}$, acting as $x \mapsto x^{q}$. This automorphism depends on the choice of the morphism $\bar{y} \rightarrow y$, but its conjugacy class is independent of any choices. Thus, we obtain a conjugacy class of automorphisms of the stalk $\mathcal{F}_{\bar{y}}$. Therefore the trace of the geometric Frobenius automorphism is canonically assigned to $\mathcal{F}$ and $y$. We will denote it by $\operatorname{Tr}\left(\operatorname{Fr}_{y}, \mathcal{F}\right)$.

More generally, if $\mathcal{F}$ is a complex of $\ell$-adic sheaves, we take the alternating sum of the traces of $\mathrm{Fr}_{y}$ on the stalk cohomologies of $\mathcal{F}$ at $\bar{y}$. Hence we obtain a function $\mathbf{f}_{\mathcal{F}, \mathbb{F}_{q}}$ on the set of $\mathbb{F}_{q}$-points of $Y$, whose value at $y \in Y\left(\mathbb{F}_{q}\right)$ is

$$
\mathbf{f}_{\mathcal{F}, \mathbb{F}_{q}}(y)=\sum_{i}(-1)^{i} \operatorname{Tr}\left(\operatorname{Fr}_{y}, H_{\bar{y}}^{i}(\mathcal{F})\right) .
$$

Similarly, for each $n>1$, we define a function $\mathbf{f}_{\mathcal{F}, \mathbb{F}_{q^{n}}}$ on the set of $\mathbb{F}_{q^{n}}$-points of $Y$ by the formula

$$
\mathbf{f}_{\mathcal{F}, \mathbb{F}_{q^{n}}}(y)=\sum_{i}(-1)^{i} \operatorname{Tr}\left(\operatorname{Fr}_{y}, H_{\bar{y}}^{i}(\mathcal{F})\right), \quad y \in Y\left(\mathbb{F}_{q^{n}}\right)
$$

(now $\operatorname{Fr}_{y}$ corresponds to the automorphism $y \mapsto y^{q^{n}}$ ).

The maps $\mathcal{F} \rightarrow \mathfrak{f}_{\mathcal{F}, \mathbb{F}_{q^{n}}}$ intertwine the natural operations on complexes of sheaves with natural operations on functions [48, Section 1.2]. For example, the pullback of a sheaf corresponds to the pullback of a function, and push-forward of a sheaf with compact support corresponds to the fiberwise integration of a function. This passage from sheaves to functions is referred to as Grothendieck's faisceaux-fonctions dictionnaire. 
If $Y=\operatorname{Bun}_{G}$, then the set of $\mathbb{F}_{q}$-points of $Y$ is naturally isomorphic to the double quotient

$$
G(F) \backslash G\left(\mathbb{A}_{F}\right) / G\left(\mathcal{O}_{F}\right)
$$

where

$$
\mathcal{O}_{F}=\prod_{x \in C} \mathcal{O}_{x}
$$

[25, Section 3.2]. Therefore, any perverse sheaf $\mathcal{F}$ on $\operatorname{Bun}_{G}$ gives rise to a function $\boldsymbol{f}_{\mathcal{F}, \mathbb{F}_{q}}$ on the double quotient (8.2). Suppose now that $\left(\mathcal{F},\left(\alpha_{V}\right)\right)$ is a Hecke eigensheaf on $\operatorname{Bun}_{G}$. Consider the corresponding function $\mathbf{f}_{\mathcal{F}, \mathbb{F}_{q}}$ on the set $\operatorname{Bun}_{G}\left(\mathbb{F}_{q}\right)$, isomorphic to the double quotient (8.2), and its transform under the Hecke functor $H_{V}$, restricted to

$$
\left(C \times \operatorname{Bun}_{G}\right)\left(\mathbb{F}_{q}\right)=C\left(\mathbb{F}_{q}\right) \times \operatorname{Bun}_{G}\left(\mathbb{F}_{q}\right)
$$

The action of the Hecke functor $H_{V}$ on sheaves becomes the action of the corresponding Hecke operators $T_{V, x}$ on functions. Hence for each $x \in C\left(\mathbb{F}_{q}\right)$, the left-hand side of (8.1) gives rise to the function $T_{V, x} \cdot \mathbf{f}_{\mathcal{F}, \mathbb{F}_{q}}$, whereas the right-hand side becomes $\operatorname{Tr}\left(\operatorname{Fr}_{x}, V_{\mathcal{E}}\right) \mathfrak{f}_{\mathcal{F}, \mathbb{F}_{q}}$. Hence the isomorphism (8.1) implies that

$$
T_{V, x} \cdot \mathbf{f}_{\mathcal{F}, \mathbb{F}_{q}}=\operatorname{Tr}\left(\operatorname{Fr}_{x}, V_{\mathcal{E}}\right) \mathbf{f}_{\mathcal{F}, \mathbb{F}_{q}}=\operatorname{Tr}\left(\sigma_{x}\left(\operatorname{Fr}_{x}\right), V\right) \mathbf{f}_{\mathcal{F}, \mathbb{F}_{q}}, \quad \forall x \in C\left(\mathbb{F}_{q}\right)
$$

(see [25, Section 3.8], for more details).

This is the sought-after Hecke eigenfunction property, but there is a caveat: a priori this condition is satisfied only for the $\mathbb{F}_{q}$-points of $C$. In contrast, an unramified Hecke eigenfunction with respect to $\sigma$ is supposed to be an eigenfunction of the Hecke operators for all closed points of $C$, with arbitrary residue fields. To ensure that this property holds for the function $\mathrm{f}_{\mathcal{F}, \mathbb{F}_{q}}$ at all points $x \in C$, we have to impose an additional condition on the perverse sheaf $\mathcal{F}$; namely, the $S_{2}$-equivariance of the iterated Hecke functor from [28, Section 1.1]. This will be discussed in the next section.

\subsection{Equivariance and commutativity conditions for Hecke eigensheaves}

Recall that the Hecke functor $H_{V}$ acts from the derived category of sheaves on $\operatorname{Bun}_{G}$ to the derived category of sheaves on $C \times \operatorname{Bun}_{G}$. Applying this functor again, we obtain the iterated Hecke functor $H_{V}^{\otimes 2}$ from the derived 
category of sheaves on $\operatorname{Bun}_{G}$ to the derived category of sheaves on $C \times$ $C \times \operatorname{Bun}_{G}$. A Hecke eigensheaf $\mathcal{F}$ with "eigenvalue" $\mathcal{E}$ is equipped with an isomorphism

$$
\alpha_{V}: H_{V}(\mathcal{F}) \simeq V_{\mathcal{E}} \otimes \mathcal{F}
$$

which gives rise to an isomorphism

$$
\alpha_{V}^{\otimes 2}: H_{V}^{\bigotimes 2}(\mathcal{F}) \simeq V_{\mathcal{E}} \otimes V_{\mathcal{E}} \otimes \mathcal{F}
$$

Away from the diagonal $\Delta \subset C \times C$ we have a natural action of the symmetric group $S_{2}$ on both sides of this isomorphism. The extra condition that we need to impose is that $\alpha_{V}^{\bigotimes 2}$ is an $S_{2}$-equivariant isomorphism.

This condition implies that for the $m$ th iterated Hecke functor $H_{V}^{\bigotimes m}$ acting from from the derived category of sheaves on $\operatorname{Bun}_{G}$ to the derived category of sheaves on $C^{m} \times \operatorname{Bun}_{G}$, the isomorphism

$$
\alpha_{V}^{\bigotimes m}: H_{V}^{\bigotimes m}(\mathcal{F}) \simeq\left(V_{\mathcal{E}}\right)^{\bigotimes m} \otimes \mathcal{F}
$$

is $S_{m}$-equivariant outside the union $\Delta$ of pairwise diagonals in $C^{m}$.

Suppose that the $S_{2}$-equivariance condition holds. Then $\mathcal{F}$ is an eigensheaf with respect to the symmetrized Hecke functor $H_{V}^{(m)}$ acting from the derived category of sheaves on $\operatorname{Bun}_{G}$ to the derived category of sheaves on $\left(\mathrm{Sym}^{m} C \backslash \Delta\right) \times \operatorname{Bun}_{G}$, that is, we have an isomorphism

$$
\alpha_{V}^{(m)}: H_{V}^{(m)}(\mathcal{F}) \simeq V_{\mathcal{E}}^{(m)} \otimes \mathcal{F}
$$

on $\left(\operatorname{Sym}^{m} C \backslash \Delta\right) \times \operatorname{Bun}_{G}$, where

$$
V_{\mathcal{E}}^{(m)}=\left(p_{*}\left(\left(V_{\mathcal{E}}\right)^{\bigotimes m}\right)\right)^{S_{m}},
$$

and $p: C^{m} \rightarrow \mathrm{Sym}^{m} C$ is the symmetrization map.

Now observe that any closed point $x$ of $C$ of degree $m$ gives rise to an $\mathbb{F}_{q}$-point $D(x)$ in $\mathrm{Sym}^{m} C$ (an effective divisor of degree $m$ ). Moreover, it is easy to see that

$$
\operatorname{Tr}\left(\operatorname{Fr}_{x}, V_{\mathcal{E}}\right)=\operatorname{Tr}\left(\operatorname{Fr}_{D(x)}, V_{\mathcal{E}}^{(m)}\right) .
$$

Therefore, restricting $\alpha_{V}^{(m)}(\mathcal{F})$ to $D(x) \times \operatorname{Bun}_{G}$ and evaluating the traces of the Frobenius on $\mathbb{F}_{q}$-points there, we find that formula (8.3) holds for all closed points $x$ of degree $m$. Thus, the $S_{2}$-equivariance condition guarantees that the function $\boldsymbol{f}_{\mathcal{F}, \mathbb{F}_{q}}$ is truly a Hecke eigenfunction on (8.2) with respect to the local system $\mathcal{E}$ (or homomorphism $\sigma: W_{F} \rightarrow{ }^{\mathrm{L}} G$ ). 
The fact that the "naive" Hecke eigensheaf property (8.1) does not by itself imply the Hecke eigenfunction property for those closed points whose residue field is a nontrivial extension of $\mathbb{F}_{q}$, the field of definition of our curve $C$, comes as a bit of a surprise. However, the $S_{2}$-equivariance that is needed to ensure that the Hecke eigenfunction property does hold everywhere is a very natural condition. In fact, it is a special case of the following general commutativity condition for the Hecke functors, introduced in [28, Section 1.4].

For $V, W \in \operatorname{Rep}\left({ }^{\mathrm{L}} G\right)$, let $H_{V}$ and $H_{W}$ be the corresponding Hecke functors from the derived category of sheaves on $\mathrm{Bun}_{G}$ to the derived category of sheaves on $C \times \operatorname{Bun}_{G}$. We then have the iterated functors $H_{V} \circ H_{W}$ from the derived category of sheaves on $\operatorname{Bun}_{G}$ to the derived category of sheaves on $C \times C \times \operatorname{Bun}_{G}$. Given a Hecke eigensheaf $\left(\mathcal{F},\left(\alpha_{V}\right)\right)$, we have isomorphisms

$$
\alpha_{V} \circ \alpha_{W}:\left(H_{V} \circ H_{W}\right)(\mathcal{F}) \simeq \mathcal{E}_{V} \otimes \mathcal{E}_{W} \otimes \mathcal{F}
$$

On the other hand, over $C \times C \backslash \Delta$ we have a natural identification

$$
\left.\left.\left(H_{V} \circ H_{W}\right)(\mathcal{F})\right|_{C \times C \backslash \Delta} \simeq \sigma^{*} \circ\left(H_{W} \circ H_{V}\right)(\mathcal{F})\right|_{C \times C \backslash \Delta},
$$

where $\sigma$ is the transposition on $C \times C \backslash \Delta$. The commutativity condition is that the diagram

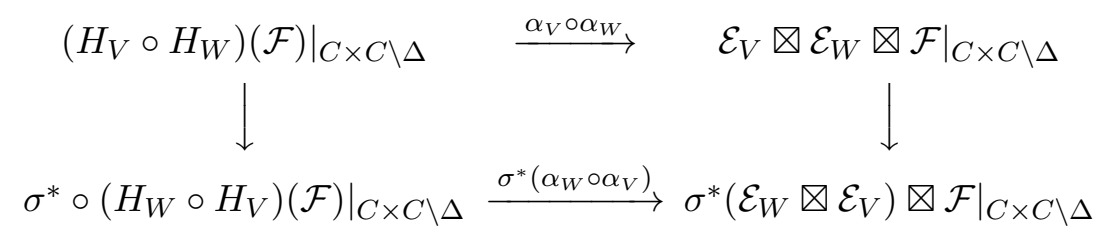

is commutative. If $V=W$, we obtain the above $S_{2}$-equivariance condition.

To explain the meaning of this commutativity condition, let us recall from [58] the geometric Satake equivalence between the category of Hecke functors supported at a fixed point $x \in C$, which is the category of equivariant perverse sheaves on the affine Grassmannian, and the category $\operatorname{Rep}\left({ }^{\mathrm{L}} G\right)$. This is an equivalence of tensor categories, which means that in addition to being compatible with the tensor products in both categories, it is also compatible with the commutativity and associativity constraints. On the former category, the commutativity constraint is defined (following Drinfeld) as a certain limit of the transposition of the Hecke functors defined at distinct points of $C$, when the points coalesce. 
The notion of (regular) Hecke eigensheaf may be viewed as a natural generalization of the notion of a fiber functor from the category of the Hecke functors supported at one point $x \in C$ to $\operatorname{Rep}\left({ }^{\mathrm{L}} G\right)$, when we allow the point $x$ to move along the curve. From this point of view, asking that the isomorphisms $\alpha_{V}$ be compatible with the tensor product structures and associativity is akin to asking for the fiber functor to be a monoidal functor (that is, one compatible with the tensor products and associativity constraint). But we know from the Tannakian theory that this is not sufficient for establishing an equivalence of a tensor category and the category of representations of an algebraic group. For that we also need the fiber functor to be compatible with the commutativity constraint. Since the commutativity constraint on the category of Hecke functors supported at one point appears as the limit the transposition of the two Hecke functors supported at two different points of $C$, the commutativity constraint itself appears as the limit of the above commutativity condition when the two points coalesce.

Therefore, we see that it is quite natural to require that a Hecke eigensheaf satisfy the commutativity condition. An interesting fact that we have observed above is that part of this condition (for $V=W$ ) is also necessary for ensuring that, when working in positive characteristic, the function associated to a Hecke eigensheaf is a Hecke eigenfunction for all closed points of the curve.

\subsection{Back to $S L_{2}$}

As we have seen in the previous section, the geometric counterpart of the double quotient (8.2) is the moduli stack $\operatorname{Bun}_{G}$ of $G$-bundles on $C$. In fact, (8.2) is the set of $\mathbb{F}_{q}$-points of $\operatorname{Bun}_{G}$. Therefore, Hecke eigensheaves on Bun $G$ give rise to Hecke eigenfunctions on (8.2), as explained above.

On the other hand, we have seen in Section 7.3 that in order to understand properly the $L$-packets of (unramified) automorphic representations for $G=S L_{2}$ we need to consider more general double quotients (7.12), where $K_{x}=K_{x}^{\prime}$ or $K_{x}^{\prime \prime}$, and $K_{x}=K_{x}^{\prime}=S L_{2}[[t]]$ for all but finite many closed points $x \in C$. If all $K_{x}=S L_{2}[[t]]$, then (7.12) is the set of $\mathbb{F}_{q}$-points of $\operatorname{Bun}_{S L_{2}}$. What about the more general quotients (7.12)? The answer is clear: these are the sets of $\mathbb{F}_{q}$-points of the "improper" versions of Bun $S L_{2}$; namely, the moduli stacks Bun $\mathcal{L}_{S L_{2}}^{\mathcal{L}}$ of rank two vector bundles with the determinant being the line bundle $\mathcal{L}=\mathcal{O}(D)$. Here $D$ is the set of points where $K_{x}=K_{x}^{\prime \prime}$, which we view as an effective divisor on $C$.

We have already encountered these moduli stacks in the case of curves over $\mathbb{C}$ in Sections 3.8, 5.1.2 and 5.2.3. At that time we remarked that if 
$\mathcal{L}=\mathcal{L}^{\prime} \otimes \mathcal{N}^{2}$, where $\mathcal{N}$ is a line bundle on $C$, then we may identify $\operatorname{Bun}_{S_{L_{2}}}^{\mathcal{L}}$ with Bun $\mathcal{L}_{S L_{2}}^{\mathcal{L}_{2}}$ by tensoring a rank 2 vector bundle with $\mathcal{N}$. Therefore, $\operatorname{Bun}_{S L_{2}}^{\mathcal{L}}$ really depends not on $\mathcal{L}$ but on its image in the quotient of the Picard group $\operatorname{Pic}(\mathbb{C})$ (which is the set of $\mathbb{C}$-points of the Picard scheme Pic of $C$ ) by the subgroup of squares. This quotient is isomorphic to $\mathbb{Z}_{2}$, and so there is a unique improper component $\operatorname{Bun}_{S L_{2}}^{\mathcal{L}}$ in this case (for which we may choose $\mathcal{L}=\mathcal{O}(p)$, where $p$ is a point of $C$ ), up to a noncanonical isomorphism.

Now consider a curve $C$ defined over $\mathbb{F}_{q}$. Here again the improper stacks Bun $\mathcal{L}_{S L_{2}}^{\mathcal{L}}$ are classified, up to an isomorphism, by the quotient $\operatorname{Pic}\left(\mathbb{F}_{q}\right) /$ $\operatorname{Pic}\left(\mathbb{F}_{q}\right)^{2}$. But now this quotient is much bigger. To describe it more precisely, let us recall [67] that the abelian class field theory identifies $\operatorname{Pic}\left(\mathbb{F}_{q}\right)$ with the maximal unramified abelian quotient of the Weil group $W_{F}$ of the function field $F$ of $C$. In other words, $\operatorname{Pic}\left(\mathbb{F}_{q}\right)$ is isomorphic to a dense subgroup of the Galois group of the maximal unramified abelian extension $F^{\mathrm{ab}, \text { un }}$ of $F$, defined in the same way as the Weil group of $F$. Namely, it is the preimage of $\mathbb{Z} \subset \widehat{\mathbb{Z}}$ under the homomorphism $\operatorname{Gal}\left(F^{\text {ab,un }} / F\right) \rightarrow$ $\operatorname{Gal}\left(\overline{\mathbb{F}}_{q} / \mathbb{F}_{q}\right)=\widehat{\mathbb{Z}}$. Therefore, we obtain that $\operatorname{Pic}\left(\mathbb{F}_{q}\right) / \operatorname{Pic}\left(\mathbb{F}_{q}\right)^{2}$ is the maximal quotient of $\operatorname{Gal}\left(F^{\mathrm{ab}, \text { un }} / F\right)$ such that all of its elements have order 2. It is also the dual group of the group of unramified quadratic extensions of $F$. Indeed, each such extension $E / F$ gives rise to a quadratic character of $\operatorname{Pic}\left(\mathbb{F}_{q}\right)$, which factors through $\operatorname{Pic}\left(\mathbb{F}_{q}\right) / \operatorname{Pic}\left(\mathbb{F}_{q}\right)^{2}$.

Let us choose a representative $\mathcal{L}=\mathcal{O}(D)$ of this group, where $D$ is a subset of the set of closed points of $C$. Then we have the algebraic moduli stack $\operatorname{Bun}_{S L_{2}}^{\mathcal{L}}$ of rank two vector bundles on $C$ with the determinant $\mathcal{L}$, whose set of $\mathbb{F}_{q}$-points is the double quotient (7.12) with the above choice of subgroups $K_{x}$. In the geometric theory, the notion of Hecke eigenfunction on this set becomes that of (regular) Hecke eigensheaf, defined in the same way as for the proper moduli stack $\operatorname{Bun}_{S L_{2}}$ (corresponding to $\mathcal{L}=\mathcal{O}$ ).

\subsection{From curves over $\mathbb{C}$ to curves over $\mathbb{F}_{q}$}

Let us go back to a curve $C$ over $\mathbb{C}$ and choose a $P G L_{2}$-local system $\mathcal{E}$ whose structure group is reduced to $O_{2} \subset P G L_{2}$, but not to its proper subgroup. Since $\mathcal{E}$ comes from an irreducible rank 2 local system, we expect that the category of regular Hecke eigensheaves (in the sense of Section 6.5) with eigenvalue $\mathcal{E}$ on $\operatorname{Bun}_{S L_{2}}$ has one irreducible object (up to an isomorphism). Let $\mathcal{F}$ be the underlying $\mathcal{D}$-module on $\operatorname{Bun}_{S L_{2}}$. In Section 5 , we have discussed the $A$-brane $\mathcal{A}$ corresponding to $\mathcal{F}$, which is represented by a rank 1 unitary local system on the singular Hitchin fiber, which has two irreducible 
components. We have observed that $\mathcal{A}$ splits into two $A$-branes, $\mathcal{A}_{+}$and $\mathcal{A}_{-}$ supported on the two irreducible components of the Hitchin fiber. Therefore, we expect that the $\mathcal{D}$-module $\mathcal{F}$ also splits into a direct sum,

$$
\mathcal{F}=\mathcal{F}_{+} \oplus \mathcal{F}_{-}
$$

of two irreducible $\mathcal{D}$-modules on $\operatorname{Bun}_{S L_{2}}$ corresponding to the two $\mathcal{A}$-branes on the singular Hitchin fiber. Moreover, since the $A$-branes $\mathcal{A}_{ \pm}$are fractional eigenbranes with respect to the 't Hooft operators, we expect that the sheaves $\mathcal{F}_{ \pm}$satisfy the fractional Hecke property introduced in Section 6.5.

This leads us to postulate that the same phenomenon should also occur for curves over a finite field $\mathbb{F}_{q}$. Namely, the regular Hecke eigensheaf $\mathcal{F}$ corresponding to an $\ell$-adic local system $\mathcal{E}$ on a curve $C$ defined over $\mathbb{F}_{q}$, of the kind discussed above, should also split as a direct sum (8.5). Moreover, these sheaves should satisfy the fractional Hecke property introduced in Section 6.5 and hence give rise to a category of fractional Hecke eigensheaves. Next, in the setting of curves over finite fields, we can pass from $\ell$-adic perverse sheaves on $\operatorname{Bun}_{S L_{2}}$, to functions. Thus, each of the sheaves $\mathcal{F}_{ \pm}$ should give rise to a function $f_{ \pm}$on the double quotient (8.2), which is the set of $\mathbb{F}_{q}$-points of $\operatorname{Bun}_{S L_{2}}$. The fractional Hecke property of the sheaves $\mathcal{F}_{ \pm}$ translates into a certain property of the corresponding functions $f_{ \pm}$.

Thus, we started with $A$-branes and ended up with automorphic functions satisfying the fractional Hecke property. Schematically, this passage looks as follows:

$$
A \text {-branes } \stackrel{\text { over } \mathbb{C}}{\Longrightarrow} \mathcal{D} \text {-modules } \stackrel{\text { over }}{\Longrightarrow} \text { perverse sheaves } \stackrel{\text { over } \mathbb{F}_{q}}{\Longrightarrow} \text { functions. }
$$

We will see below that the fractional Hecke property means in particular that not only $f_{+}+f_{-}$is also a Hecke eigenfunction, in the ordinary sense, but $f_{+}-f_{-}$is also a Hecke eigenfunction as well, but with respect to a different homomorphism $\sigma^{\prime}: W_{F} \rightarrow P G L_{2}$. We will show that $\sigma^{\prime}$ really exists, and is in fact canonically attached to the original homomorphism $\sigma$. This will provide the first consistency check for our predictions.

\subsection{Fractional Hecke property}

Let $C$ be a curve over $\mathbb{F}_{q}$ and $\mathcal{E}$ an endoscopic $\ell$-adic $P G L_{2}$-local system on $C$ (corresponding to an unramified homomorphism $\sigma: W_{F} \rightarrow P G L_{2}$ ). This means that its structure group is reduced to $\mathrm{O}_{2}$, but not to a proper subgroup. Then the group of automorphisms of $\mathcal{E}$ (equivalently, the centralizer of the image of $\sigma$ ) is $\mathbb{Z}_{2}$. Let $D$ be a finite set of closed points of $C$. 
Denote by $\mathcal{F}^{D}$ a regular Hecke eigensheaf on $\operatorname{Bun}_{S L_{2}}^{\mathcal{O}(D)}$ with the "eigenvalue" $\mathcal{E}$ (in the sense of Section 6.5). Motivated by our results on $A$-branes in the analogous situation for curves over $\mathbb{C}$, we conjecture that $\mathcal{F}^{D}$ splits as a direct sum

$$
\mathcal{F}^{D}=\mathcal{F}_{+}^{D} \oplus \mathcal{F}_{-}^{D}
$$

of perverse sheaves $\mathcal{F}_{ \pm}^{D}$ which satisfy the following fractional Hecke property with respect to $\mathcal{E}$, introduced in Section 6.5 (and so we also call them the fractional Hecke eigensheaves):

$$
\begin{aligned}
& \alpha_{+}: H_{W}\left(\mathcal{F}_{+}^{D}\right) \stackrel{\sim}{\longrightarrow}\left(\operatorname{det} U_{\mathcal{E}} \otimes \mathcal{F}_{+}^{D}\right) \oplus\left(U_{\mathcal{E}} \otimes \mathcal{F}_{-}^{D}\right) \\
& \alpha_{-}: H_{W}\left(\mathcal{F}_{-}^{D}\right) \stackrel{\sim}{\longrightarrow}\left(U_{\mathcal{E}} \otimes \mathcal{F}_{+}^{D}\right) \oplus\left(\operatorname{det} U_{\mathcal{E}} \otimes \mathcal{F}_{-}^{D}\right)
\end{aligned}
$$

Here $W$ is the adjoint representation of $P G L_{2}$ and we use the decomposition of the rank 3 local system $W_{\mathcal{E}}$ on $C$ with respect to the action of its group $\mathbb{Z}_{2}$ of automorphisms as in formula (6.8),

$$
W_{\mathcal{E}}=\left(\operatorname{det} U_{\mathcal{E}} \otimes I\right) \oplus\left(U_{\mathcal{E}} \otimes S\right)
$$

where $I$ and $S$ are the trivial and sign representations of $\mathbb{Z}_{2}$, respectively, and $\operatorname{det} U_{\mathcal{E}}$ and $U_{\mathcal{E}}$ are the rank 1 and 2 local systems on $C$ defined as follows. Recall that by our assumption the $P G L_{2}$-local system $\mathcal{E}$ is reduced to $\mathrm{O}_{2}$, so we view it as an $\mathrm{O}_{2}$-local system. We then set

$$
U_{\mathcal{E}}=\mathcal{E} \underset{\mathrm{O}_{2}}{\times} U
$$

where, as before, $U$ is the defining two-dimensional representation of $\mathrm{O}_{2}$.

\subsection{Fractional Hecke eigenfunctions}

We now analyze the implications of formulas (8.7) and (8.8) for the functions associated to $\mathcal{F}_{ \pm}^{D}$

$$
f_{ \pm}^{D}=\mathbf{f}_{\mathcal{F}_{ \pm}^{D}, \mathbb{F}_{q}}
$$

on the set $\operatorname{Bun}_{S L_{2}}^{\mathcal{O}(D)}\left(\mathbb{F}_{q}\right)$, which is isomorphic to the double quotient (7.12). Formula (8.6) implies that

$$
f^{D}=f_{+}^{D}+f_{-}^{D}
$$


where $f^{D}=\mathbf{f}_{\mathcal{F}^{D}, \mathbb{F}_{q}}$ is the function on $\operatorname{Bun}_{S L_{2}}^{\mathcal{O}(D)}\left(\mathbb{F}_{q}\right)$ associated to the regular Hecke eigensheaf $\mathcal{F}^{D}$.

To simplify our notation, in what follows, when no ambiguity arises, we will suppress the upper index $D$.

By restricting the Hecke correspondence to $x \times \operatorname{Bun}_{S L_{2}}^{\mathcal{O}(D)}$, where $x \in$ $C\left(\mathbb{F}_{q}\right)$ and evaluating the trace of the Frobenius at the $\mathbb{F}_{q}$-points there, we obtain from formulas (8.7) and (8.8) that the functions $f_{ \pm}$satisfy the following property:

$$
T_{W, x} \cdot\left(\begin{array}{l}
f_{+} \\
f_{-}
\end{array}\right)=\left(\begin{array}{ll}
a_{x} & b_{x} \\
b_{x} & a_{x}
\end{array}\right)\left(\begin{array}{l}
f_{+} \\
f_{-}
\end{array}\right), x \in C\left(\mathbb{F}_{q}\right),
$$

where

$$
\begin{aligned}
a_{x} & =\operatorname{Tr}\left(\operatorname{Fr}_{x}, \operatorname{det} U_{\mathcal{E}}\right)=\operatorname{det}\left(\sigma_{x}\left(\operatorname{Fr}_{x}\right), U\right), \\
b_{x} & =\operatorname{Tr}\left(\operatorname{Fr}_{x}, U_{\mathcal{E}}\right)=\operatorname{Tr}\left(\sigma_{x}\left(\operatorname{Fr}_{x}\right), U\right)
\end{aligned}
$$

Here we view $\sigma_{x}$ as a homomorphism $W_{F_{x}} \rightarrow O_{2}$. To compute these numbers, we recall the description of the Frobenius conjugacy classes from Section 7.2. We find that the conjugacy class of $\sigma_{x}\left(\mathrm{Fr}_{x}\right)$ in $O_{2}$ contains the matrix

$$
\left(\begin{array}{cc}
\frac{\mu\left(\operatorname{Fr}_{y_{1}(x)}\right)}{\mu\left(\operatorname{Fr}_{y_{2}(x)}\right)} & 0 \\
0 & \frac{\mu\left(\operatorname{Fr}_{y_{2}(x)}\right)}{\mu\left(\operatorname{Fr}_{y_{1}(x)}\right)}
\end{array}\right)
$$

if $x$ is split, and the matrix

$$
\left(\begin{array}{rr}
1 & 0 \\
0 & -1
\end{array}\right)
$$

if $x$ is nonsplit. Therefore, we find that

$$
\begin{aligned}
& a_{x}=\left\{\begin{array}{cl}
1, & \text { if } x \text { is split } \\
-1, & \text { if } x \text { is nonsplit, }
\end{array}\right. \\
& b_{x}= \begin{cases}\frac{\mu\left(\operatorname{Fr}_{y_{1}(x)}\right)}{\mu\left(\operatorname{Fr}_{y_{2}(x)}\right)}+\frac{\mu\left(\operatorname{Fr}_{y_{2}(x)}\right)}{\mu\left(\operatorname{Fr}_{y_{1}(x)}\right)}, & \text { if } x \text { is split, } \\
0, & \text { if } x \text { is nonsplit, }\end{cases}
\end{aligned}
$$

Formula (8.11) implies that the sum $f_{+}+f_{-}$is an eigenfunction of the Hecke operators

$$
T_{W, x} \cdot\left(f_{+}+f_{-}\right)=\left(a_{x}+b_{x}\right)\left(f_{+}+f_{-}\right), \quad x \in C\left(\mathbb{F}_{q}\right),
$$


where

$$
\begin{aligned}
a_{x}+b_{x}=\operatorname{Tr}\left(\operatorname{Fr}_{x}, W_{\mathcal{E}}\right) & =\operatorname{Tr}\left(\sigma_{x}\left(\operatorname{Fr}_{x}\right), W\right) \\
& = \begin{cases}1+\frac{\mu\left(\operatorname{Fr}_{y_{1}(x)}\right)}{\mu\left(\operatorname{Fr}_{y_{2}(x)}\right)}+\frac{\mu\left(\operatorname{Fr}_{y_{2}(x)}\right)}{\mu\left(\operatorname{Fr}_{y_{1}(x)}\right)}, & \text { if } x \text { is split, } \\
-1, & \text { if } x \text { is nonsplit }\end{cases}
\end{aligned}
$$

Thus, formula (8.14) expresses the usual Hecke property of the function $f=f_{+}+f_{-}$associated to the sheaf $\mathcal{F}=\mathcal{F}_{+} \oplus \mathcal{F}_{-}$with respect to $\mathcal{E}$ (or $\sigma$ ).

But we also find that the difference $f_{+}-f_{-}$is a Hecke eigenfunction with a different set of eigenvalues; namely,

$$
T_{W, x} \cdot\left(f_{+}-f_{-}\right)=\left(a_{x}-b_{x}\right)\left(f_{+}-f_{-}\right), x \in C\left(\mathbb{F}_{q}\right),
$$

where

$$
a_{x}-b_{x}= \begin{cases}1-\frac{\mu\left(\operatorname{Fr}_{y_{1}(x)}\right)}{\mu\left(\operatorname{Fr}_{y_{2}(x)}\right)}-\frac{\mu\left(\operatorname{Fr}_{y_{2}(x)}\right)}{\mu\left(\operatorname{Fr}_{y_{1}(x)}\right)}, & \text { if } x \text { is split } \\ -1, & \text { if } x \text { is nonsplit }\end{cases}
$$

However, we have to remember that the Hecke property (8.16) holds only for $\mathbb{F}_{q}$-points of $C$. Indeed, we have started with the geometric Hecke properties (8.7) and (8.8). The Hecke correspondence relates $\operatorname{Bun}_{S L_{2}}^{\mathcal{O}(D)}$ and $C \times \operatorname{Bun}_{S L_{2}}^{\mathcal{O}(D)}$. The functions $f_{ \pm}$are obtained from $\mathcal{F}_{ \pm}$by taking the trace of the Frobenius at the $\mathbb{F}_{q}$-points of $\operatorname{Bun}_{S L_{2}}^{\mathcal{O}(D)}$. To obtain a Hecke property for them, we need to consider the Hecke correspondence on the sets of $\mathbb{F}_{q}$-points of these two stacks. The only Hecke operators we can reach this way are those corresponding to the $\mathbb{F}_{q}$-points of $C$. The resulting action of the Hecke operators is expressed by Equation (8.11).

This formula does not uniquely determine the function $f_{+}-f_{-}$. It would be uniquely determined (at least for generic $\sigma$ 's of the type we are considering, which correspond to irreducible two-dimensional representations of $W_{F}$ ) only if it were a Hecke eigenfunction for all closed points of $C$, not just its $\mathbb{F}_{q}$-points.

In order to incorporate closed points of $C$ with the residue field $\mathbb{F}_{q^{m}}$, $m>1$, we need to consider more general Hecke correspondence $H_{W}^{(m)}$ over the $m$ th symmetric power of $C$ (with the union $\Delta$ of pairwise diagonals removed). This requires an additional $S_{2}$-equivariance condition on the isomorphisms $\alpha_{ \pm}$, similar to the one in the case of regular Hecke eigensheaves. This condition is defined in exactly the same way as in Section 8.2. 
Assuming that this $S_{2}$-equivariance condition holds, we obtain isomorphisms

$$
\begin{aligned}
\alpha_{+}^{(2)}: H_{W}^{(2)}\left(\mathcal{F}_{+}\right) \stackrel{\sim}{\longrightarrow} & \left.\left(\left(\operatorname{det} U_{\mathcal{E}}\right)^{(2)} \oplus U_{\mathcal{E}}^{(2)}\right)\right|_{\operatorname{Sym}^{2} C \backslash \Delta} \otimes \mathcal{F}_{+} \\
& \left.\oplus \operatorname{Sym}\left(\operatorname{det} U_{\mathcal{E}} \otimes U_{\mathcal{E}} \oplus U_{\mathcal{E}} \otimes \operatorname{det} U_{\mathcal{E}}\right)\right|_{\operatorname{Sym}^{2} C \backslash \Delta} \otimes \mathcal{F}_{-}, \\
\alpha_{-}^{(2)}: H_{W}^{(2)}\left(\mathcal{F}_{-}\right) \stackrel{\sim}{\longrightarrow} & \left.\operatorname{Sym}\left(\operatorname{det} U_{\mathcal{E}} \otimes U_{\mathcal{E}} \oplus U_{\mathcal{E}} \otimes \operatorname{det} U_{\mathcal{E}}\right)\right|_{\operatorname{Sym}^{2} C \backslash \Delta} \\
& \left.\nabla \mathcal{F}_{+} \oplus\left(\left(\operatorname{det} U_{\mathcal{E}}\right)^{(2)} \oplus U_{\mathcal{E}}^{(2)}\right)\right|_{\operatorname{Sym}^{2} C \backslash \Delta} \otimes \mathcal{F}_{-} .
\end{aligned}
$$

Here we use notation (8.4).

Suppose that this condition is satisfied. Recall from Section 8.2 that any closed point $x$ of $C$ such that $\operatorname{deg}(x)=m$ (for the definition of $\operatorname{deg}(x)$, see Section 7.1) gives rise to an $\mathbb{F}_{q}$-point $D(x)$ in $\operatorname{Sym}^{m} C$ (an effective divisor of degree $m$ ), and we have

$$
\operatorname{Tr}\left(\operatorname{Fr}_{D(x)}, \mathcal{E}^{(m)}\right)=\operatorname{Tr}\left(\operatorname{Fr}_{x}, \mathcal{E}\right)
$$

for any local system $\mathcal{E}$ on $C$. In particular, suppose that $x$ is a closed point of $C$ of degree 2, that is, with the residue field isomorphic to $\mathbb{F}_{q^{2}}$, and let $D(x)$ be the corresponding $\mathbb{F}_{q}$-point of $\operatorname{Sym}^{2} C$. Then we have

$$
\operatorname{Tr}\left(\operatorname{Fr}_{D(x)}, \operatorname{det} U_{\mathcal{E}}^{(2)} \oplus U_{\mathcal{E}}^{(2)}\right)=\operatorname{Tr}\left(\operatorname{Fr}_{D(x)}, W_{\mathcal{E}}^{(2)}\right)=\operatorname{Tr}\left(\operatorname{Fr}_{x}, W_{\mathcal{E}}\right)=a_{x}+b_{x}
$$

but

$$
\operatorname{Tr}\left(\operatorname{Fr}_{D(x)}, \operatorname{Sym}\left(\operatorname{det} U_{\mathcal{E}} \otimes U_{\mathcal{E}} \oplus U_{\mathcal{E}} \otimes \operatorname{det} U_{\mathcal{E}}\right)\right)=0
$$

Therefore, the isomorphisms $\alpha_{ \pm}^{(2)}$ imply that both $f_{+}$and $f_{-}$are Hecke eigenfunctions (in the ordinary sense) with the eigenvalue $\left(a_{x}+b_{x}\right)$, and so we have

$$
T_{W, x} \cdot\left(f_{+}-f_{-}\right)=\left(a_{x}+b_{x}\right)\left(f_{+}-f_{-}\right), \quad x \in C, \quad \operatorname{deg}(x)=2 .
$$

Next, we analyze in the same way what happens at the closed points of $C$ of arbitrary degree $m$. The $S_{2}$-invariance condition implies the $S_{m}$-invariance condition, as in Section 8.2. We then find that for odd $m$ the function $f_{+}-f_{-}$satisfies formula (8.16), and for even $m$ it satisfies formula (8.18). Thus, we have

$$
T_{W, x} \cdot\left(f_{+}-f_{-}\right)=\left(a_{x}+(-1)^{m} b_{x}\right)\left(f_{+}-f_{-}\right), \quad x \in C, \quad \operatorname{deg}(x)=m .
$$


According to the Langlands correspondence for $G L_{2}[22,23]$, a formula like this may only hold if the eigenvalues of $T_{W, x}$, the numbers $a_{x}+$ $(-1)^{m} b_{x}$, are equal to $\operatorname{Tr}\left(\sigma_{x}^{\prime}\left(\mathrm{Fr}_{x}, W\right)\right.$ for some homomorphism $\sigma^{\prime}: W_{F} \rightarrow$ $P G L_{2}$. This gives us an opportunity to test our prediction that there exists a decomposition (8.6).

In fact, it is easy to construct a homomorphism $\sigma^{\prime}$ with this property. Observe that any homomorphism $\sigma: W_{F} \rightarrow O_{2}$ may be twisted by a quadratic character $\rho: W_{F} \rightarrow \mathbb{Z}_{2}=\{ \pm 1\}$, where the group $\mathbb{Z}_{2}$ is identified with the center of $\mathrm{O}_{2}$. We denote this operation by $\sigma \mapsto \sigma \otimes \rho$.

In particular, the quadratic extension of the scalars $\mathbb{F}_{q^{2}} / \mathbb{F}_{q}$ defines a quadratic extension $\mathbb{F}_{q^{2}}(C) / \mathbb{F}_{q}(C)$ (recall that $F=\mathbb{F}_{q}(C)$ ) and hence a quadratic character of $W_{F}$, which we will denote by $\nu$. This character is determined by the following property:

$$
\nu\left(\operatorname{Fr}_{x}\right)=(-1)^{m}, \quad x \in C, \quad \operatorname{deg}(x)=m
$$

Let $\sigma^{\prime}=\sigma \otimes \nu$. Then we find that

$\operatorname{Tr}\left(\sigma_{x}^{\prime}\left(\operatorname{Fr}_{x}\right), W\right)= \begin{cases}1+(-1)^{m} \frac{\mu\left(\operatorname{Fr}_{y_{1}(x)}\right)}{\mu\left(\operatorname{Fr}_{y_{2}(x)}\right)}+(-1)^{m} \frac{\mu\left(\operatorname{Fr}_{y_{2}(x)}\right)}{\mu\left(\operatorname{Fr}_{y_{1}(x)}\right)}, & \text { if } x \text { is split, } \\ -1, & \text { if } x \text { is nonsplit }\end{cases}$

for all $x \in C\left(\mathbb{F}_{q}\right)$. Hence

$$
\operatorname{Tr}\left(\sigma_{x}^{\prime}\left(\operatorname{Fr}_{x}\right), W\right)=a_{x}+(-1)^{m} b_{x}
$$

Therefore, we obtain that a Hecke eigenfunction with the eigenvalues $a_{x}+$ $(-1)^{m} b_{x}$, as in formula (8.19), does exist. Let us denote this function by $f^{\prime}$.

Using this function, we can now solve for $f_{+}$and $f_{-}$:

$$
f_{ \pm}=\frac{1}{2}\left(f \pm f^{\prime}\right)
$$

These are the functions corresponding to the fractional Hecke eigensheaves $\mathcal{F}_{ \pm}$whose existence we have conjectured. ${ }^{44}$

It is natural to ask: what is the representation theoretical meaning of the functions $f_{ \pm}$and the equations that they satisfy? These equations are

\footnotetext{
${ }^{44}$ After this paper appeared on the arXiv, we learned from Lysenko $[56,57]$ that the sheaves $\mathcal{F}_{ \pm}$may be constructed using his results on theta-lifting.
} 
given by formula (8.11) for $x \in C$ of odd degree $m$, and

$$
T_{W, x} \cdot f_{ \pm}=\left(a_{x}+b_{x}\right) f_{ \pm}
$$

for $x \in C$ of even degree $m$.

Recall that the Hecke eienfunctions, such as $f=f_{+}+f_{-}$and $f^{\prime}=f_{+}-$ $f_{-}$, may be interpreted as matrix coefficients of automorphic representations of $S L_{2}\left(\mathbb{A}_{F}\right)$, and their (regular) Hecke property is the result of the Satake isomorphism identifying the spherical Hecke algebra with the representation ring of the Langlands dual group $P G L_{2}$. It would be interesting to find a similar interpretation of the fractional Hecke eigenfunctions $f_{ \pm}$and equations (8.11) and (8.22).

\subsection{The improper Hecke functors}

In addition to the "proper" Hecke functors $H_{W}$ acting on the categories of $\mathcal{D}$-modules on $\operatorname{Bun}_{S L_{2}}^{\mathcal{O}(D)}$, there are also "improper" Hecke functors $\widetilde{H}_{x}$ acting from the category of $\mathcal{D}$-modules on $\operatorname{Bun}_{S L_{2}}^{\mathcal{O}(D)}$ to the category of $\mathcal{D}$-modules on $\operatorname{Bun}_{S L_{2}}^{\mathcal{O}(D+x)}$. They are defined via the Hecke correspondence between the two moduli stacks consisting of pairs of rank 2 bundles $\mathcal{M} \in \operatorname{Bun}_{S L_{2}}^{\mathcal{O}(D)}$ and $\mathcal{M}^{\prime} \in \operatorname{Bun}_{S L_{2}}^{\mathcal{O}(D+x)}$ such that $\mathcal{M} \subset \mathcal{M}^{\prime}$ as a coherent sheaf. These functors are categorical analogs of the improper Hecke operators $\widetilde{T}_{x}$ introduced in Section 7.4. The corresponding operators on $A$-branes are the improper 't Hooft operators discussed in Section 5.3.

In formula (5.38), we have computed the action of the improper 't Hooft operators on the branes $\mathcal{A}_{ \pm}$. Based in this formula, we conjecture that the improper Hecke operators should act on the fractional Hecke eigensheaves $\mathcal{F}_{ \pm}^{D}$ as follows:

$$
\begin{aligned}
\widetilde{H}_{x}\left(\mathcal{F}_{+}^{D}\right) & \simeq \mathcal{F}_{+}^{D+x} \oplus \mathcal{F}_{-}^{D+x} \\
\widetilde{H}_{x}\left(\mathcal{F}_{-}^{D}\right) & \simeq \mathcal{F}_{+}^{D+x} \oplus \mathcal{F}_{-}^{D+x}
\end{aligned}
$$

This should hold for all points $x \in C$ if $C$ is defined over $\mathbb{C}$, and all split points, if $C$ is defined over $\mathbb{F}_{q}$.

This formula indicates that the improper Hecke functor fails to establish an equivalence between the categories of fractional Hecke eigensheaves on $\operatorname{Bun}_{S L_{2}}^{\mathcal{O}(D)}$ and $\operatorname{Bun}_{S L_{2}}^{\mathcal{O}(D+x)}$ for the endoscopic local systems. This may be viewed as a geometric counterpart of the vanishing of the improper Hecke operator acting on functions discussed in Section 7.4, which, as we have seen, 
is closely related to the structure of the global $L$-packets of automorphic representations associated to endoscopic $\sigma: W_{F} \rightarrow P G L_{2}$.

It would be more difficult to see an analog of the phenomenon of $L$-packets in the framework of the categories of regular Hecke eigensheaves. Indeed, for a regular Hecke eigensheaf $\mathcal{F}^{D}=\mathcal{F}_{+}^{D} \oplus \mathcal{F}_{-}^{D}$, we have

$$
\widetilde{H}_{x}\left(\mathcal{F}^{D}\right) \simeq V \otimes \mathcal{F}^{D+x}
$$

where $V$ is a two-dimensional vector space. In the classical setting explained in Section 7.4, $V$ is replaced by the trace $\operatorname{Tr}\left(\widetilde{\sigma}_{x}\left(\mathrm{Fr}_{x}\right), V\right)$. It vanishes precisely when $\sigma_{x}\left(\mathrm{Fr}_{x}\right)$ is the conjugacy class of (7.5), and this vanishing manifests the nontrivial structure of the $L$-packets in this case. In contrast, in the geometric setting, $V$ itself appears as the multiplier in formula (8.23). It is not clear what should replace the vanishing of the trace in this context.

However, in the framework of the categories of fractional Hecke eigensheaves, the picture is more transparent. Now we have a category with two irreducible objects, $\mathcal{F}_{+}^{D}$ and $\mathcal{F}_{-}^{D}$. The functor $\widetilde{H}_{x}$ sends both of them to $\mathcal{F}^{D+x}=\mathcal{F}_{+}^{D+x} \oplus \mathcal{F}_{-}^{D+x}$, and hence does not set up an equivalence between the categories corresponding to $D$ and $D+x$.

\section{8. $L$-packets associated to $\sigma$ and $\sigma^{\prime}$}

The above discussion shows that the representations $\pi=\bigotimes_{x}^{\prime} \pi_{x}$ of $S L_{2}(\mathbb{A})$ corresponding to $\sigma: W_{F} \rightarrow O_{2} \subset P G L_{2}$ and $\sigma^{\prime}=\sigma \otimes \nu$, where $\nu$ is given by formula (8.20), are linked together. Let us compare the $L$-packets (or, equivalently, the multiplicities of the representations $\pi$ ) corresponding to $\sigma$ and $\sigma^{\prime}$.

Recall that for each finite subset $D \subset C$ we have the space of $\prod_{x} K_{x^{-}}$ invariant vectors in $\pi$, where $K_{x}=K_{x}^{\prime \prime}$, if $x \in D$, and $K_{x}=K_{x}^{\prime}$, otherwise. If $\pi$ is automorphic, then this space of invariants is realized in the space of Hecke eigenfunctions on the double quotient (7.12), which is $\operatorname{Bun}_{S L_{2}}^{\mathcal{O}(D)}\left(\mathbb{F}_{q}\right)$. As explained in Section 7.3, this space is either one- or zero-dimensional. In the former case, there is a unique Hecke eigenfunction (up to a scalar), which we denote by $f_{\sigma}^{D}$. In the latter case, any Hecke eigenfunction that we construct has to vanish. A criterion as to whether it is one- or zerodimensional is given in Theorem 7.1 (following [42]), and it amounts to a description of the global $L$-packets.

This criterion is as follows. Consider the case when we can lift $\sigma$ to $\tilde{\sigma}: W_{F} \rightarrow G L_{2}$ and represent $\widetilde{\sigma}$ as $\operatorname{Ind}_{W_{E}}^{W_{F}} \mu$, where $E$ is the field of functions on a double covering $C^{\prime} \rightarrow C$, and $\mu$ is a character of $W_{E}$. Then let $S \subset D$ 
be the set of points in $D$ which are nonsplit in $E$. The dimension of the space of Hecke eigenfunctions on $\operatorname{Bun}_{S L_{2}}^{\mathcal{O}(D)}\left(\mathbb{F}_{q}\right)$ with respect to $\sigma$ is then equal to 1 , if $\# S$ is even, and to 0 , if $\# S$ is odd.

Note that the extension $E / F$ corresponds to the quadratic character of $W_{F}$ obtained via the composition $\kappa: W_{F} \stackrel{\sigma}{\longrightarrow} O_{2} \rightarrow \mathbb{Z}_{2}$. We will call $E$ the field affiliated with $\sigma$.

Lemma 8.1. There exists a quadratic character $\phi: W_{E} \rightarrow \mathbb{Z}_{2}$, where $E$ is affiliated with $\sigma$, such that $\sigma^{\prime}=\sigma \otimes \nu$ is equivalent to the projectivization of the two-dimensional representation $\operatorname{Ind}_{W_{E}}^{W_{F}}(\mu \otimes \phi)$ of $W_{F}$.

Proof. Let us choose $\widetilde{\tau} \in W_{F}$ which projects onto the nontrivial element $\tau$ of $W_{F} / W_{E}=\mathbb{Z}_{2}$. By Cebotarev's theorem, the condition stated in the lemma is equivalent to saying that $\phi$ satisfies

$$
\frac{\phi\left(\widetilde{\tau} h \widetilde{\tau}^{-1}\right)}{\phi(h)}=\nu(h), \quad h \in W_{E} .
$$

The existence of such $\phi$ may be derived from the Hasse-Minkowski theorem, as was explained to us by Poonen. We will not present his argument here, as this would take us too far afield.

Since the same quadratic extension is affiliated with both $\sigma$ and $\sigma^{\prime}$, we find that the criterion for the dimensionality of the space of Hecke eigenfunctions on $\operatorname{Bun}_{S L_{2}}^{\mathcal{O}(D)}\left(\mathbb{F}_{q}\right)$ with respect to $\sigma$ and $\sigma^{\prime}$ is the same. Therefore, the Hecke eigenfunctions $f_{\sigma}^{D}$ and $f_{\sigma^{\prime}}^{D}$ have to vanish simultaneously when $\# S$ is odd, where $S \subset D$ is the subset of points of $D$ which are nonsplit in $E$.

Now recall that the functions $f_{\sigma, \pm}^{D}$, corresponding to the sheaves $\mathcal{F}_{\sigma, \pm}^{D}$ on $\operatorname{Bun}_{S L_{2}}^{\mathcal{O}(D)}$, are given by formula (8.21),

$$
f_{\sigma, \pm}^{D}=\frac{1}{2}\left(f_{\sigma}^{D} \pm f_{\sigma^{\prime}}^{D}\right)
$$

Hence we find that both of these functions have to vanish if $\# S$ is odd.

Therefore, we obtain that for odd \#S the fractional Hecke eigensheaves $\mathcal{F}_{\sigma, \pm}^{D}$ on $\operatorname{Bun}_{S L_{2}}^{\mathcal{O}(D)}$ are such that the corresponding functions $f_{\sigma, \pm}^{D}$ on the set of $\mathbb{F}_{q}$-points of $\operatorname{Bun}_{S L_{2}} \mathcal{O}(D)$ are identically equal to 0 . However, this does not mean that the sheaves themselves are equal to 0 . This only means that the traces of the Frobenius on the stalks of $\mathcal{F}_{\sigma, \pm}^{D}$ at the $\mathbb{F}_{q}$-points of $\operatorname{Bun}_{S L_{2}} \mathcal{O}_{(D)}$ are equal to 0 . But this does not mean that the traces of the Frobenius on the stalks at $\mathbb{F}_{q^{n}}$-points are equal to 0 for $n>1$ (which would have implied 
that the sheaves are identically zero [48]). In fact, it is easy to see that the latter are nonzero for general $n$.

Before explaining this, we note a general fact about compatibility of Hecke eigensheaves with base change. For each $n>1$, we have the curve

$$
C_{n}=C \underset{\operatorname{Spec} \mathbb{F}_{q}}{\times} \operatorname{Spec} \mathbb{F}_{q^{n}}
$$

over $\mathbb{F}_{q^{n}}$. The moduli stack $\operatorname{Bun}_{G, C_{n}}$ of $G$-bundles on $C_{n}$ is equivalent to the base change of the moduli stack $\operatorname{Bun}_{G}=\operatorname{Bun}_{G, C}$ of $G$-bundles on $C$,

$$
\operatorname{Bun}_{G, C_{n}}=\operatorname{Bun}_{G} \underset{\text { Spec } \mathbb{F}_{q}}{\times} \operatorname{Spec} \mathbb{F}_{q^{n}}
$$

Let $\mathcal{E}_{n}$ be the pullback of the ${ }^{\mathrm{L}} G$-local system $\mathcal{E}$ on $C$ to $C_{n}$. The geometric Langlands correspondence is compatible with base change, in the sense that the pullback $\mathcal{F}_{n}$ of a Hecke eigensheaf $\mathcal{F}$ with eigenvalue $\mathcal{E}$ from $\operatorname{Bun}_{G, C}$ to $\operatorname{Bun}_{G, C_{n}}$ is a Hecke eigensheaf with the eigenvalue $\mathcal{E}_{n}[49]$.

Let us consider now the traces of the Frobenius on the stalks of our sheaves $\mathcal{F}_{\sigma, \pm}^{D}$ at $\mathbb{F}_{q^{m}}$-points of $\operatorname{Bun}_{S L_{2}, C} \mathcal{O}^{(D)}$ with $m>1$. As an example, let us look at the set of $\mathbb{F}_{q^{2}}$-points of $\operatorname{Bun}_{S L_{2}, C}$, which is is the same as the set of $\mathbb{F}_{q^{2}}$-points of the moduli stack $\operatorname{Bun}_{S L_{2}, C_{2}}$ of $S L_{2}$-bundles on $C_{2}$. The pullback of a Hecke eigensheaf $\mathcal{F}_{\sigma}^{D}$ to $\operatorname{Bun}_{S L_{2}, C_{2}}^{\mathcal{O}(D)}$ is a Hecke eigensheaf $\mathcal{F}_{\sigma_{2}}^{D}$, where $\sigma_{2}$ is the restriction of $\sigma$ to $W_{F_{2}}$, with $F_{2}=\mathbb{F}_{q^{2}}(C)$. Therefore, the pullback of $\mathcal{F}_{\sigma, \pm}^{D}$ is $\mathcal{F}_{\sigma_{2}, \pm}^{D}$. Suppose, for example, that $D=[y]$, where $y$ is

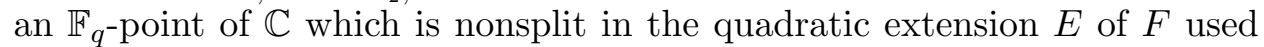
in defining $\sigma$. Then the set $S$ appearing in the statement of Theorem 7.1 consists of a single point $y$, and according to this theorem, the functions $f_{\sigma, \pm}^{[y]}$ on the set $\operatorname{Bun}_{S L_{2}, C}^{\mathcal{O}(y)}\left(\mathbb{F}_{q}\right)$ associated to $\mathcal{F}_{\sigma, \pm}^{D}$ have to vanish.

However, the $\mathbb{F}_{q^{2}}$-point of $C_{2}$ corresponding to $y$ (which we will also denote by $y$ ) is split in the corresponding quadratic extension $E_{2}$ of $F_{2}$. Therefore, by Theorem 7.1, the functions $f_{\sigma_{2}, \pm}^{D}$ on $\operatorname{Bun}_{S L_{2}, C_{2}}^{\mathcal{O}(y)}\left(\mathbb{F}_{q^{2}}\right)=$ $\operatorname{Bun}_{S L_{2}, C}^{\mathcal{O}(y)}\left(\mathbb{F}_{q^{2}}\right)$ are nonzero. According to the above compatibility property with base change, these functions coincide with the functions on $\operatorname{Bun}_{S L_{2}, C} \mathcal{O}(D)$ $\left(\mathbb{F}_{q^{2}}\right)$ corresponding to the sheaves $\mathcal{F}_{\sigma, \pm}^{[y]}$. Hence the sheaves themselves are nonzero.

This elementary example shows that even if the functions on the set of $\mathbb{F}_{q}$-points of $\operatorname{Bun}_{S L_{2}, C} \mathcal{O}(D)$ associated to the sheaves $\mathcal{F}_{\sigma, \pm}^{D}$ are equal to zero, the corresponding functions on the sets of $\mathbb{F}_{q^{m}}$-points with $m>1$, are not all equal to zero simultaneously, and hence the sheaves $\mathcal{F}_{\sigma, \pm}^{D}$ are nonzero. 


\subsection{Abelian case}

In Section 6.6, we have discussed other examples of fractional Hecke eigensheaves. We now revisit them in the case when the underlying curve $C$ is defined over $\mathbb{F}_{q}$. It is instructive to look at the corresponding functions on the sets of $\mathbb{F}_{q}$-points of $\operatorname{Bun}_{G}$ and to express them in terms of the ordinary Hecke eigenfunctions, the way we did in the endoscopic example for $G=S L_{2}$ above (see formula (8.24)).

Consider first the case when $G$ is a one-dimensional torus. The corresponding moduli space, the Picard variety Pic, breaks into connected components $\mathrm{Pic}_{n}, n \in \mathbb{Z}$, and the Hecke eigensheaf $\mathcal{F}_{\sigma}$ corresponding to a one-dimensional ( $\ell$-adic) representation $\sigma$ of the Weil group $W_{F}$, breaks into a direct sum

$$
\mathcal{F}_{\sigma}=\bigoplus_{n \in \mathbb{Z}} \mathcal{F}_{\sigma, n}
$$

where $\mathcal{F}_{\sigma, n}$ is supported on $\mathrm{Pic}_{n}$. This is an analog of decomposition (8.6). Let $f_{\sigma}$ (resp., $f_{\sigma, n}$ ) be the function on $\operatorname{Pic}\left(\mathbb{F}_{q}\right)$ (resp., $\operatorname{Pic}_{n}\left(\mathbb{F}_{q}\right)$ ) corresponding to $\mathcal{F}_{\sigma}$ (resp., $\mathcal{F}_{\sigma, n}$ ). Then we have

$$
f_{\sigma}=\sum_{n \in \mathbb{Z}} f_{\sigma, n} .
$$

This is analogous to formula (8.10). We now wish to express the functions $f_{\sigma, n}$ in terms of (ordinary) Hecke eigenfunctions $f_{\sigma^{\prime}}$, similar to formula (8.24).

This is achieved by a simple Fourier transform. Namely, for each nonzero number $\gamma \in \mathbb{C}^{\times}$(in what follows we identify $\overline{\mathbb{Q}}_{\ell}$ with $\mathbb{C}$ ) we define a one-dimensional representation $\alpha_{\gamma}$ of $W_{F}$ as the composition of the homomorphism

$$
\text { res }: W_{F} \rightarrow W_{\mathbb{F}_{q}}=\mathbb{Z}
$$

obtained by restricting to the scalars $\mathbb{F}_{q} \subset F$, and the homomorphism

$$
\mathbb{Z} \rightarrow \mathbb{C}^{\times}, \quad 1 \mapsto \gamma
$$

Now let $\sigma_{\gamma}=\sigma \otimes \alpha_{\gamma}$ be the twist of $\sigma$ by $\alpha_{\gamma}$. Then we have the following obvious formula

$$
f_{\sigma, n}=\frac{1}{2 \pi i} \int_{|\gamma|=1} f_{\sigma_{\gamma}} \gamma^{-n-1} d \gamma
$$


expressing the functions $f_{\sigma, n}$ as integrals of the ordinary Hecke eigenfunctions corresponding to the twists $\sigma_{\gamma}$ of $\sigma$ by $\alpha_{\gamma},|\gamma|=1$.

Formula (8.27) is an analog of formula (8.24) which we had in the endoscopic case, in the sense that in both cases the functions satisfying the fractional Hecke property (that is, $f_{\sigma, \pm}$ in the endoscopic case, and $f_{\sigma, n}, n \in \mathbb{Z}$, in the abelian case) are expressed via Fourier transform of ordinary Hecke eigenfunctions. The difference is that in the first case the Fourier transform is performed with respect to the finite group $\mathbb{Z}_{2}$, which is the group of automorphisms of an endoscopic homomorphism $\sigma: W_{F} \rightarrow P G L_{2}$, whereas in the second case it is performed with respect to a continuous group of automorphisms (or rather, its compact form $U_{1}$ ). This is the reason why a finite sum in (8.24) is replaced by an integral in (8.27). We will see other examples of this kind of Fourier transform with respect to more general finite groups of automorphisms in Section 9.4.

In a similar way, we can obtain functions satisfying the fractional Hecke property associated to other types of local systems discussed in Section 6.6: for more general tori, for local systems whose group of automorphisms is the center of ${ }^{\mathrm{L}} G$ and for the Eisenstein series. It would be interesting to find a direct representation-theoretic interpretation of these functions and the fractional Hecke property that they satisfy.

\subsection{The Iwahori case}

We have discussed above the Hecke eigensheaves on the moduli stacks $\operatorname{Bun}_{S L_{2}}^{\mathcal{O}(D)}$ and the corresponding Hecke eigenfunctions. However, in our most detailed example of $A$-branes corresponding to the elliptic curves in Section 3, we have considered a slightly different moduli space corresponding to ramified Higgs bundles. In this case, the relevant moduli stack is $\operatorname{Bun}_{S L_{2}, I_{p}}^{\mathcal{O}(D)}$ which parametrizes rank two vector bundles on $C$ with determinant $\mathcal{O}(D)$ and a parabolic structure at a fixed point $p$ of $C$ (that is, a choice of a line in the fiber of the bundle at $p$ ). It is instructive to look at how the story with $L$-packets discussed in Section 7.3 plays out in this case.

Let $C$ be again defined over $\mathbb{F}_{q}$. Then the set of $\mathbb{F}_{q}$-points of $\operatorname{Bun}_{S L_{2}, I_{p}}^{\mathcal{O}(D)}$ is isomorphic to the double quotient

$$
S L_{2}(F) \backslash S L_{2}\left(\mathbb{A}_{F}\right) /\left(\prod_{x \neq p} K_{x} \times I_{p}\right) .
$$

Here $I_{p}=K_{p}^{\prime} \cap K_{p}^{\prime \prime}$ is the Iwahori subgroup of $S L_{2}\left(F_{p}\right)$, and $K_{x}=K_{x}^{\prime \prime}$ for $x \in D, K_{x}=K_{x}^{\prime}$, otherwise. Let us suppose that $p$ is a nonsplit point of $C$, 
with respect to the unramified covering $C^{\prime} \rightarrow C$ affiliated with an unramified homomorphism $\sigma: W_{F} \rightarrow O_{2}$. Then the local $L$-packet corresponding to $p$ and a homomorphism $\sigma: W_{F} \rightarrow P G L_{2}$ constructed as above consists of two irreducible representations, $\pi_{p}^{\prime}$ and $\pi_{p}^{\prime \prime}$, but now both $\left(\pi_{p}^{\prime}\right)^{I_{p}}$ and $\left(\pi_{p}^{\prime \prime}\right)^{I_{p}}$ are one-dimensional.

Let us fix the local factors $\pi_{x}, x \neq p$. Then we have two nonisomorphic irreducible representations of $S L_{2}\left(\mathbb{A}_{F}\right)$,

$$
\bigotimes_{x \neq p} \pi_{x} \otimes \pi_{p}^{\prime} \quad \text { and } \bigotimes_{x \neq p} \pi_{x} \otimes \pi_{p}^{\prime \prime}
$$

According to Theorem 7.1, only one of them is automorphic, that is, may be realized as a constituent of an appropriate space of functions on $S L_{2}(F) \backslash$ $S L_{2}\left(\mathbb{A}_{F}\right)$. However, their spaces of invariants with respect to the subgroup

$$
\prod_{x \neq p} K_{x} \times I_{p}
$$

are both one-dimensional. Therefore, no matter which one of them is automorphic, we will have a one-dimensional space of Hecke eigenfunctions on the double quotient (8.28). Thus, the function on the set of $\mathbb{F}_{q}$-points of $\operatorname{Bun}_{S L_{2}, I_{p}}^{\mathcal{O}(D)}$ associated to a regular Hecke eigensheaf will be nonzero. In the same way as above, we then obtain that the functions $f_{ \pm}^{D}$ associated to fractional Hecke eigensheaves are also nonzero in this case. This constitutes an important difference between the double quotients (7.12) and (8.28).

\section{Other groups}

In this section, we sketch a generalization of our results and conjectures to the case of an arbitrary semi-simple simply connected Lie group $G$ (the latter assumption is not essential and is made to simplify the exposition). Then ${ }^{\mathrm{L}} G$ is a semi-simple Lie group of adjoint type.

\subsection{Overview}

Recall that we have two dual moduli spaces of Higgs bundles, $\mathcal{M}_{\mathrm{H}}(G)$ and $\mathcal{M}_{\mathrm{H}}\left({ }^{\mathrm{L}} G\right)$, and the corresponding dual Hitchin fibrations (2.2). The geometric Langlands correspondence is interpreted in [39] as the homological mirror symmetry between these two moduli spaces that reduces to the fiberwise 
$T$-duality on generic fibers which are smooth dual tori. Under this mirror symmetry, the categories of $B$-branes on $\mathcal{M}_{\mathrm{H}}(G)$ and $A$-branes on $\mathcal{M}_{\mathrm{H}}\left({ }^{\mathrm{L}} G\right)$ are supposed to be equivalent. We are interested in the $A$-branes on $\mathcal{M}_{\mathrm{H}}\left({ }^{\mathrm{L}} G\right)$ corresponding to the $B$-branes supported at the orbifold singular points of $\mathcal{M}_{\mathrm{H}}\left({ }^{\mathrm{L}} G\right)$. Such a singular point may be viewed as an ${ }^{\mathrm{L}} G$-local system $\mathcal{E}$ with a nontrivial, but finite, group of automorphisms $\Gamma$. We call such a local system "elliptic endoscopic," or simply "endoscopic," for brevity. Then $\mathcal{E}$ is reduced to one or more of the dual (elliptic) endoscopic subgroups ${ }^{\mathrm{L}} H \subset{ }^{\mathrm{L}} G$, which are defined as the centralizers of nontrivial elements of $\Gamma$.

The category of $B$-branes (or, equivalently, coherent sheaves) supported at such a point $\mathcal{E}$ is equivalent to the category $\operatorname{Rep}(\Gamma)$ of representations of $\Gamma$. The objects of the corresponding category of $A$-branes are supported on the Hitchin fiber $\boldsymbol{F}_{b}$ in $\mathcal{M}_{\mathrm{H}}(G)$ dual to the Hitchin fiber ${ }^{\mathrm{L}} \boldsymbol{F}_{b}$ in $\mathcal{M}_{\mathrm{H}}\left({ }^{\mathrm{L}} G\right)$ containing the point $\mathcal{E}$. Thus, $b$ is the image of $\mathcal{E} \in \mathcal{M}_{\mathrm{H}}\left({ }^{\mathrm{L}} G\right)$ in the Hitchin base $\boldsymbol{B}$. The question that we take up in this section is to describe the categories of $A$-branes corresponding to the elliptic endoscopic ${ }^{\mathrm{L}} G$-local systems and their properties under the action of the 't Hooft/Hecke operators.

In the previous sections, we analyzed in detail the case of $G=S L_{2}$. In this case, the only dual elliptic endoscopic subgroup is $O_{2} \subset S O_{3}={ }^{\mathrm{L}} G$, and generic local systems which are reduced to $\mathrm{O}_{2}$ have the automorphism group $\Gamma=\mathbb{Z}_{2}$. The corresponding category of $B$-branes is equivalent to $\operatorname{Rep}\left(\mathbb{Z}_{2}\right){ }^{45}$ On the $A$-model side, this corresponds to the fact that the Hitchin fiber $\boldsymbol{F}_{b}$ has two irreducible components. Therefore, the dual category of $A$-branes has two irreducible objects supported on those components. These fractional $A$-branes have additional parameters; namely, rank 1 unitary local systems, which correspond to $\mathrm{O}_{2}$-local systems. Thus, we obtain a concrete realization of the transfer (also known as the functoriality principle, or, in the physics interpretation, the domain wall phenomenon) corresponding to the homomorphism $\mathrm{O}_{2} \rightarrow \mathrm{SO}_{3}$ in the geometric setting, as explained in Section 5.2.6. Finally, the two fractional $A$-branes satisfy the fractional Hecke property, as explained in Section 5.3. In Section 8, we have interpreted these results for $A$-branes in terms of the corresponding $\mathcal{D}$-modules on $\operatorname{Bun}_{S L_{2}}$ and automorphic functions when the curve $C$ is defined over a finite field (see (1.1)). In this section, we propose a generalization of this picture.

\footnotetext{
${ }^{45}$ This equivalence is noncanonical due to the twist by a gerbe described below in Section 10, but we will ignore this issue for now.
} 


\subsection{Categories of branes corresponding to the endoscopic local systems}

Let $G$ be a semi-simple simply connected complex Lie group, and ${ }^{\mathrm{L}} G$ its Langlands dual group (of adjoint type, with the trivial center).

An ${ }^{\mathrm{L}} G$-local system $\mathcal{E}$ will be called elliptic endoscopic, or simply endoscopic, if its group of automorphisms is a nontrivial finite group, which we will denote by $\Gamma$. The dual endoscopic groups ${ }^{\mathrm{L}} H_{s}$ associated with such a local system ${ }^{46}$ are by definition the centralizers of nontrivial elements $s \in \Gamma, s \neq 1$. The structure group of $\mathcal{E}$ may be reduced to any of the ${ }^{\mathrm{L}} H_{s}$. As we have already pointed out in Section 5.2.6, for any subgroup ${ }^{\mathrm{L}} H \subset{ }^{\mathrm{L}} G$ we have a natural inclusion $\mathcal{M}_{\mathrm{H}}\left({ }^{\mathrm{L}} H\right) \subset \mathcal{M}_{\mathrm{H}}\left({ }^{\mathrm{L}} G\right)$. Therefore, we see that an endoscopic local system $\mathcal{E}$ lies in the intersection of the images of $\mathcal{M}_{\mathrm{H}}\left({ }^{\mathrm{L}} H_{s}\right), s \in \Gamma, s \neq 1$, in $\mathcal{M}_{\mathrm{H}}\left({ }^{\mathrm{L}} G\right)$.

Note that $\mathcal{E} \in \mathcal{M}_{\mathrm{H}}\left({ }^{\mathrm{L}} G\right)$ may also be viewed as a Higgs bundle (in the complex structure $I$ ). Its group of automorphisms as a Higgs bundle will also be $\Gamma$, and this Higgs bundle will be reduced to the subgroups ${ }^{\mathrm{L}} H_{s}, s \in \Gamma$.

An endoscopic local system $\mathcal{E}$, viewed as a point of the moduli space $\mathcal{M}_{\mathrm{H}}\left({ }^{\mathrm{L}} G\right)$, is an orbifold point. Denote by $B$-branes $\mathcal{E}$ the category of $B$-branes (coherent sheaves) supported at $\mathcal{E}$. This category is equivalent to $\operatorname{Rep}(\Gamma)$, although there may not be a canonical equivalence, as explained in Section 10 below (in that case, let us choose such an equivalence). Then for each representation $R$ of $\Gamma$, we have a $B$-brane $\mathcal{B}_{R}$ in the category $B$-branes $\mathcal{E}$. In the same way as in Section 5.3.2, we find that the action of the Wilson operators $W_{V, p}$, where $V \in \operatorname{Rep}\left({ }^{\mathrm{L}} G\right)$ and $p \in C$, on these branes is given by the formula

$$
W_{V, p} \cdot \mathcal{B}_{R}=\sum_{R^{\prime} \in \operatorname{Irrep}(\Gamma)} V\left(R^{\prime}\right)_{\mathcal{E}_{p}} \otimes \mathcal{B}_{R^{\prime} \otimes R}, \quad R \in \operatorname{Rep}(\Gamma)
$$

Here we use the decomposition of $V$ with respect to the action of ${ }^{\mathrm{L}} G \times \Gamma$,

$$
V=\bigoplus_{R^{\prime} \in \operatorname{Irrep}(\Gamma)} V\left(R^{\prime}\right) \otimes R^{\prime}
$$

\footnotetext{
${ }^{46}$ Here we follow the tradition of calling $H_{s}$ rather than ${ }^{\mathrm{L}} H_{s}$ the endoscopic groups.
} 
where $\operatorname{Irrep}(\Gamma)$ is the set of equivalence classes of irreducible representations of $\Gamma$. Also, for any representation $U$ of ${ }^{\mathrm{L}} G$, we use the notation

$$
U_{\mathcal{E}_{p}}=\mathcal{E}_{p} \underset{{ }_{\mathrm{L}} G}{\times} U,
$$

where $\mathcal{E}_{p}$ is the ${ }^{\mathrm{L}} G$-torsor which is the fiber of $\mathcal{E}$ at $p \in C$.

Formula (9.1) implies that the eigenbranes of the Wilson operators are direct sums of copies of the $B$-brane corresponding to the regular representation

$$
\operatorname{Reg}(\Gamma)=\bigoplus_{R \in \operatorname{Irrep}\left({ }^{\mathrm{L}} G\right)} R^{*} \otimes R
$$

of $\Gamma$, where $R^{*}$ is the dual of $R$. This $B$-brane is

$$
\mathcal{B}_{\operatorname{Reg}(\Gamma)}=\bigoplus_{R \in \operatorname{Irrep}\left({ }^{(\mathrm{L}} G\right)} R^{*} \otimes \mathcal{B}_{R} .
$$

We have

$$
W_{V, p} \cdot \mathcal{B}_{\operatorname{Reg}(\Gamma)}=V_{\mathcal{E}_{p}} \otimes \mathcal{B}_{\operatorname{Reg}(\Gamma)} .
$$

Now we consider the mirror dual category $A$-branes $\mathcal{E}$ of $A$-branes on $\mathcal{M}_{\mathrm{H}}(G)$. By analogy with the case of $G=S L_{2}$ that was explained in detail in the previous sections, we expect that these $A$-branes are supported on the Hitchin fiber $\boldsymbol{F}_{b}$ in $\mathcal{M}_{\mathrm{H}}(G)$, where $b$ is the image of $\mathcal{E} \in \mathcal{M}_{\mathrm{H}}\left({ }^{\mathrm{L}} G\right)$ in the Hitchin base $\boldsymbol{B}$. In the case of $G=S L_{2}$, we saw that for an endoscopic local system $\mathcal{E}$ different $A$-branes correspond to different components of $\boldsymbol{F}_{b}$. We would like to understand what happens in general.

One complication is that it is quite possible that there are points on the Hitchin fiber ${ }^{\mathrm{L}} \boldsymbol{F}_{b}$ which correspond to ${ }^{\mathrm{L}} G$-local systems with infinite groups of automorphisms. An example is the zero fiber ${ }^{\mathrm{L}} \boldsymbol{F}_{0}$ at $0 \in \boldsymbol{B}$, which is the nilpotent cone. Points of this fiber may have either finite or infinite groups of automorphisms. For instance, for ${ }^{\mathrm{L}} \mathrm{G}=\mathrm{SO}_{3}$, it includes the trivial local system, for which $\Gamma=S_{3}$, as well as local systems that reduce to the subgroup $\mathbb{Z}_{2} \times \mathbb{Z}_{2}$, for which $\Gamma=\mathbb{Z}_{2} \times \mathbb{Z}_{2}$, and also irreducible local systems.

If the Hitchin fiber ${ }^{\mathrm{L}} \boldsymbol{F}_{b}$ contains local systems with infinite automorphism groups, then we cannot expect that the structure of the dual Hitchin fiber $\boldsymbol{F}_{b}$ is controlled by endoscopic points of ${ }^{\mathrm{L}} \boldsymbol{F}_{b}$. Indeed, the same $\boldsymbol{F}_{b}$ would carry objects of the categories of $A$-branes mirror dual to the categories of $B$-branes supported at those local systems. Therefore, the structure of $\boldsymbol{F}_{b}$ 
should be more complicated in this case. We hope to discuss this more general case elsewhere, but for now we will restrict ourselves to the situation when infinite groups of automorphisms do not occur.

For $S L_{n}$, a useful condition that ensures that automorphism groups are finite is that the spectral curve is reduced and irreducible. This is equivalent to requiring that the characteristic polynomial of the Higgs field $\varphi$ is irreducible (which in particular implies that $\varphi(x)$ is regular semi-simple for generic $x \in C$ ). This criterion has a simple analog 47 for any $G$ which, however, is stronger than needed to ensure that automorphism groups are finite. A weaker criterion is given by Ngô in [64, Definition 7.5]. By [64, Corollaire 7.6], it is equivalent to the following. Let $\boldsymbol{P}_{b}$ be the generalized Prym variety associated to $b$, defined in [64, Section 4] (in the case when $G=S L_{2}$, this is the Prym variety of the spectral curve associated to $b$ discussed in Section 5.2.2). Then the condition is that the group $\pi_{0}\left(\boldsymbol{P}_{b}\right)$ of components of $\boldsymbol{P}_{b}$ is finite. Following [65], we write $\boldsymbol{B}^{\text {ani }}$ for the corresponding locus in $\boldsymbol{B}$.

Let us suppose then that $b \in \boldsymbol{B}^{\text {ani }}$. In this case, the Hitchin fiber is reduced and contains an open dense subset which is a torsor over the abelian group $\boldsymbol{P}_{b}$ [64]. Thus, the set of irreducible components of $\boldsymbol{F}_{b}$ is a torsor over the group $\pi_{0}\left(\boldsymbol{P}_{b}\right)$ of components of $\boldsymbol{P}_{b}$, and all components of $\boldsymbol{F}_{b}$ have multiplicity one. The group $\pi_{0}\left(\boldsymbol{P}_{b}\right)$ is finite (by our assumption that $b \in$ $\boldsymbol{B}^{\text {ani }}$ ) and abelian.

Now recall from [39] that for generic $b \in \boldsymbol{B}$ the $A$-branes corresponding to any rank 1 unitary local system on $\boldsymbol{F}_{b}$ (which is a smooth torus) are eigenbranes of the 't Hooft operators that are dual to the Wilson operators acting on the $B$-branes (see Section 5.3). We conjecture that the same is true for any $b \in \boldsymbol{B}^{\text {ani }}$. In addition, we conjecture that each irreducible object $\mathcal{A}_{R}$ of the category $A$-branes $\mathcal{E}$ corresponding to the irreducible object $\mathcal{B}_{R}$ of $B$-branes $\mathcal{E}_{\mathcal{E}}$ under the equivalence $A$-branes $_{\mathcal{E}} \simeq B$-branes $\mathcal{E}$ is supported on a union of irreducible components of $\boldsymbol{F}_{b}$.

In particular, suppose that $\Gamma=\Gamma_{b}$ is the largest possible group of automorphisms among the local systems in the dual Hitchin fiber ${ }^{\mathrm{L}} \boldsymbol{F}_{b}$. Then it

\footnotetext{
${ }^{47}$ Regard $\varphi$ as a matrix in the adjoint representation and set $P(y)=\operatorname{det}(y-\varphi)$. For $G$ simple of rank $r$, we have generically $P(y)=y^{r} Q(r)$ if $Q$ is simply laced; the condition we want is then that $Q$ is irreducible. For $G$ not simply laced, generically $P(y)=y^{r} Q(r) R(r)$, where $Q(r)$ and $R(r)$ are contributions from long and short roots, respectively. In this case, the condition is that $Q$ or equivalently $R$ should be irreducible. Note that if $(E, \varphi)$ is a Higgs bundle such that $\varphi$ obeys this criterion of irreducibility, then $(E, \varphi)$ is automatically stable; $E$ has no nontrivial $\varphi$-invariant subsheaves and hence no destabilizing ones. So over this locus, one can apply Hecke operators while working with stable Higgs bundles only.
} 
is natural to expect that each $\mathcal{A}_{R}$ is supported on a particular irreducible component of $\boldsymbol{F}_{b}$ and that there is a bijection (perhaps, noncanonical, as for $\left.G=S L_{2}\right)$ between $\operatorname{Irrep}\left(\Gamma_{b}\right)$ and the set of irreducible components of $\boldsymbol{F}_{b}$, and hence the set $\pi_{0}\left(\boldsymbol{P}_{b}\right)$. But $\pi_{0}\left(\boldsymbol{P}_{b}\right)$ is an abelian group. This suggests that $\operatorname{Irrep}\left(\Gamma_{b}\right)$ also has a natural abelian group structure and that $\Gamma_{b}$ is in fact an abelian group that is dual to $\pi_{0}\left(\boldsymbol{P}_{b}\right)$. Thus, we arrive at the following conjecture.

Conjecture 9.1. Let $\mathcal{E}$ be an elliptic endoscopic ${ }^{\mathrm{L}} G$-local system with the group of automorphisms $\Gamma$ such that the image $b$ of $\mathcal{E}$ in $\boldsymbol{B}$ lies in $\boldsymbol{B}^{\text {ani }}$. Then the group $\Gamma$ is abelian and its dual group $\widehat{\Gamma}$ may be identified with a quotient of the group $\pi_{0}\left(\boldsymbol{P}_{b}\right)$ of components of the generalized Prym variety $\boldsymbol{P}_{b}$ corresponding to $b$.

Furthermore, if $\Gamma_{b}$ is the largest group of automorphisms of the local systems in the dual Hitchin fiber ${ }^{\mathrm{L}} \boldsymbol{F}_{b}$, then $\Gamma_{b}$ is isomorphic to the dual group of $\pi_{0}\left(\boldsymbol{P}_{b}\right)$.

The results presented in Section 5 confirm this conjecture in the case when $G=S L_{2}$ (see also the footnote on page 10).

\subsection{Fractional eigenbranes and eigensheaves}

Let $\mathcal{E}$ be an endoscopic ${ }^{\mathrm{L}} G$-local system. We have the mirror dual categories $B$-branes $\mathcal{E}_{\mathcal{E}}$ and $A$-branes $\mathcal{E}_{\mathcal{E}}$ discussed in the previous subsection. The former is equivalent to $\operatorname{Rep}(\Gamma)$ and contains irreducible objects $\mathcal{B}_{R}$ attached to irreducible representations $R$ of $\Gamma$. The corresponding $A$-branes are denoted by $\mathcal{A}_{R}$. Therefore, the $B$-brane (9.3) corresponds to the $A$-brane

$$
\mathcal{A}_{\operatorname{Reg}(\Gamma)}=\bigoplus_{R \in \operatorname{Irrep}\left({ }^{\mathrm{L}} G\right)} R^{*} \otimes \mathcal{A}_{R}
$$

In light of Conjecture 9.1, in the case when the projection of $\mathcal{E}$ onto $\boldsymbol{B}$ is in $\boldsymbol{B}^{\text {ani }}$, this decomposition should reflect the decomposition of the Hitchin fiber $\boldsymbol{F}_{b}$ into (unions of) irreducible components.

Since the $B$-brane $\mathcal{B}_{\operatorname{Reg}(\Gamma)}$ is an eigenbrane of the Wilson operators (see formula (9.4)), $\mathcal{A}_{\operatorname{Reg}(\Gamma)}$ should be an eigenbrane of the 't Hooft operators:

$$
T_{V, p} \cdot \mathcal{A}_{\operatorname{Reg}(\Gamma)}=V_{\mathcal{E}_{p}} \otimes \mathcal{A}_{\operatorname{Reg}(\Gamma)}
$$

We call $\mathcal{A}_{\operatorname{Reg}(\Gamma)}$ the regular eigenbrane. 
Furthermore, formula (9.1) for the action of the Wilson operators on the $B$-branes $B_{R}, R \in \operatorname{Irrep}(\Gamma)$, implies the following formula for the action of the 't Hooft operators $T_{V, p}, V \in \operatorname{Rep}\left({ }^{\mathrm{L}} G\right)$, on the corresponding $A$-branes $\mathcal{A}_{R}$ :

$$
T_{V, p} \cdot \mathcal{A}_{R}=\sum_{R^{\prime} \in \operatorname{Irrep}(\Gamma)} V\left(R^{\prime}\right)_{\mathcal{E}_{p}} \otimes \mathcal{A}_{R^{\prime} \otimes R}, \quad R \in \operatorname{Rep}(\Gamma) .
$$

We call the $A$-branes $\mathcal{A}_{R}$ the fractional eigenbranes.

Note that we expect formulas (9.6) and (9.7) to hold regardless of whether the projection of $\mathcal{E}$ onto $\boldsymbol{B}$ lies in $\boldsymbol{B}^{\text {ani }}$. If it is, then we expect $\Gamma$ to be abelian (see Conjecture 9.1 ); otherwise, it may well be non-abelian, as can be seen from explicit examples.

As explained in Section 2.3, we expect that to each $A$-brane on $\mathcal{M}_{\mathrm{H}}(G)$ one may associate a $\mathcal{D}$-module on $\operatorname{Bun}_{G}$. Furthermore, the properties of the $A$-branes under the action of the 't Hooft operators should translate to similar properties of the corresponding $\mathcal{D}$-modules under the action of the Hecke operators. Therefore, we predict that any Hecke eigensheaf on Bun $\mathrm{B}_{G}$ with the eigenvalue $\mathcal{E}$ (which is an endoscopic local system) is a direct sum of copies of the following $\mathcal{D}$-module:

$$
\mathcal{F}_{\operatorname{Reg}(\Gamma)}=\bigoplus_{R \in \operatorname{Irrep}(\mathrm{L} G)} R^{*} \otimes \mathcal{F}_{R}
$$

satisfying the regular Hecke property

$$
H_{V}\left(\mathcal{F}_{\operatorname{Reg}(\Gamma)}\right) \simeq V_{\mathcal{E}} \otimes \mathcal{F}_{\operatorname{Reg}(\Gamma)}
$$

(see formula $(6.5)$ ). Furthermore, we predict that its constituents $\mathcal{F}_{R}$ are irreducible $\mathcal{D}$-modules on $\operatorname{Bun}_{G}$ which satisfy an analog of formula (9.7),

$$
H_{V}\left(\mathcal{F}_{R}\right) \simeq \sum_{R^{\prime} \in \operatorname{Irrep}(\Gamma)} V\left(R^{\prime}\right)_{\mathcal{E}} \otimes \mathcal{F}_{R^{\prime} \otimes R}, \quad R \in \operatorname{Rep}(\Gamma)
$$

This is a variant of formula (6.10), which means that the $\mathcal{D}$-modules $\mathcal{F}_{R}, R \in$ $\operatorname{Irrep}(\Gamma)$, satisfy the fractional Hecke property and hence are fractional Hecke eigensheaves. In the case when $G=S L_{2}$ these are the $\mathcal{D}$-modules $\mathcal{F}_{ \pm}$ discussed in Section 6.5, and formula (9.9) coincides with formula (6.11).

Thus, we obtain a concrete conjecture about the structure of (regular) Hecke eigensheaves corresponding to endoscopic local systems: they split into direct sums of irreducible $\mathcal{D}$-modules satisfying the fractional Hecke 
property (9.9). We have derived this conjecture from the homological mirror symmetry of the dual Hitchin fibrations, using the passage from $A$-branes to $\mathcal{D}$-modules. Alternatively, one may look at it from the point of view of a non-abelian version of the Fourier-Mukai transform [51,66], suggested by Beilinson and Drinfeld, which is supposed to be an equivalence of certain categories (whose precise definition is presently unknown) of $\mathcal{O}$-modules on the moduli stack of ${ }^{\mathrm{L}} G$-local systems on $C$ and $\mathcal{D}$-modules on $\operatorname{Bun}_{G}$ (see, e.g., [25, Section 6.2]).

\subsection{Computations with Hecke eigenfunctions}

In the previous section, we have made conjectures about the structure of Hecke eigensheaves corresponding to endoscopic local systems. So far, we have discussed local systems on a complex curve $C$. However, we conjecture that the same pattern will also hold if we consider instead $\ell$-adic local systems defined on a curve over a finite field, or equivalently, $\ell$-adic homomorphisms $\sigma: W_{F} \rightarrow{ }^{\mathrm{L}} G$, where $W_{F}$ is the Weil group of the function field $F$ of this curve. Then the analog of the group $\Gamma$ is the centralizer of the image of $\sigma$, which is traditionally denoted by $S_{\sigma}$. But here we will stick to the same notation $\Gamma$. In this context, there is a new feature; namely, the Grothendieck faisceaux-fonctions dictionnaire. This enables us to pass from Hecke eigensheaves (which are now viewed as perverse sheaves on $\operatorname{Bun}_{G}$ ) to the corresponding automorphic functions on a double quotient of the adèlic group $G\left(\mathbb{A}_{F}\right)$ and gives us an opportunity to test our conjectures. We have already done this in the case when $G=S L_{2}$ in Section 8.6 and shown that such functions indeed exist. Here we extend our analysis to the general situation considered above.

9.4.1. Abelian case Suppose that we have an endoscopic homomorphism $\sigma: W_{F} \rightarrow{ }^{\mathrm{L}} G$. This means that the centralizer $\Gamma$ of its image is a nontrivial finite group (we are still under the assumption that ${ }^{\mathrm{L}} G$ is a semi-simple group of adjoint type). Let us start with the case when $\Gamma$ is abelian. Recall that in a similar situation over $\mathbb{C}$ we expect to have the irreducible $\mathcal{D}$ modules labeled (perhaps slightly noncanonically) by one-dimensional representations (characters) of $\Gamma$. Thus, we have a $\mathcal{D}$-module $\mathcal{F}_{\chi}$ for each $\chi \in \widehat{\Gamma}=\operatorname{Irrep}(\Gamma)$. They have to satisfy the fractional Hecke property (9.9). After passing to curves over a finite field, we should have the corresponding perverse sheaves on $\operatorname{Bun}_{G}$ satisfying the same property, to which we associate automorphic functions $f_{\chi}$. The fractional Hecke property for the 
sheaves translates to the following equations on these functions:

$$
T_{V, x} \cdot f_{\chi}=\sum_{\mu \in \widehat{\Gamma}} a_{V, \mu, x} f_{\chi \cdot \mu}, \quad \chi \in \widehat{\Gamma} .
$$

Here $V$ is a representation of ${ }^{\mathrm{L}} G$, which decomposes as follows

$$
V=\bigoplus_{\mu \in \widehat{\Gamma}} V(\mu)
$$

under the action of $\Gamma$, and

$$
a_{V, \mu, x}=\operatorname{Tr}\left(\sigma\left(\operatorname{Fr}_{x}\right), V(\mu)\right)
$$

where $\sigma: W_{F} \rightarrow{ }^{\mathrm{L}} G$ is the object replacing the local system $\mathcal{E}$ (since $\sigma$ lands in the centralizer of $\Gamma$, by our assumption, the right-hand side is well defined). $T_{V, x}$ is a classical Hecke operator corresponding to a closed point $x \in C$ (see Section 7.1).

We will now show that functions $f_{\chi}$ satisfying the fractional Hecke property (9.10) do exist and may be obtained by a kind of Fourier transform over $\Gamma$ from the ordinary Hecke eigenfunctions. This will generalize the formulas obtained in Section 8.6 in the case of $G=S L_{2}$.

For simplicity, we will assume here that $x$ is a closed point of $C$ with the residue field $\mathbb{F}_{q}$ equal to the ground field. For other closed points, the computation needs to be modified along the lines of Section 8.6.

From (9.10), by doing Fourier transform on $\Gamma$, we find the eigenfunctions of $T_{V, x}$ :

$$
\widehat{f}_{\gamma}=\sum_{\chi \in \widehat{\Gamma}} \chi(\gamma) f_{\chi}, \quad \gamma \in \Gamma
$$

with the eigenvalues

$$
A_{x, \gamma}=\sum_{\mu \in \widehat{\Gamma}} \mu(\gamma) a_{V, \mu, x}
$$

In particular,

$$
A_{x, 1}=\sum_{\mu \in \widehat{\Gamma}} a_{V, \mu, x}=\operatorname{Tr}\left(\sigma\left(\operatorname{Fr}_{x}\right), V\right)
$$


So

$$
\widehat{f_{1}}=\sum_{\chi \in \widehat{\Gamma}} f_{\chi}
$$

is a Hecke eigenfunction corresponding to $\sigma$, as expected.

But what about the other functions $\widehat{f}_{\gamma}$ with $\gamma \neq 1$ ? We claim that they are also Hecke eigenfunctions, but corresponding to other homomorphisms

$$
\sigma_{\gamma}: W_{F} \rightarrow{ }^{\mathrm{L}} G
$$

Namely, recall that we have a homomorphism

$$
\text { res }: W_{F} \rightarrow W_{\mathbb{F}_{q}}=\mathbb{Z}
$$

by restricting to the scalars $\mathbb{F}_{q} \subset F$. Let

$$
\alpha_{\gamma}: W_{F} \rightarrow \Gamma
$$

be the homomorphism given by the composition of res and the homomorphism $\mathbb{Z} \rightarrow \Gamma$ sending $1 \mapsto \gamma$.

Since $\Gamma$ centralizes the image of $\sigma$, the formula

$$
\sigma_{\gamma}(g)=\sigma(g) \alpha_{\gamma}(g)
$$

defines a homomorphism $W_{F} \rightarrow{ }^{\mathrm{L}} G$, for each $\gamma \in \Gamma$. We claim that

$$
A_{x, \gamma}=\operatorname{Tr}\left(\sigma_{\gamma}\left(\operatorname{Fr}_{x}\right), V\right)
$$

and so the function $\widehat{f}_{\gamma}$ is in fact a Hecke eigenfunction corresponding to $\sigma_{\gamma}: W_{F} \rightarrow{ }^{\mathrm{L}} G$ ! (We recall that in the above computation we have assumed that $x$ is an $\mathbb{F}_{q}$-point of $C$. For other closed points we obtain the same result by applying the analysis of Section 8.6.)

Now, making the inverse Fourier transform, we express the functions $f_{\chi}$ corresponding to the sheaves $\mathcal{F}_{\chi}$ in terms of the ordinary Hecke eigenfunctions:

$$
f_{\chi}=\frac{1}{|\Gamma|} \sum_{\gamma \in \Gamma} \chi(\gamma) \widehat{f}_{\gamma}
$$

This generalizes our formula

$$
f_{ \pm}=\frac{1}{2}\left(f_{\sigma} \pm f_{\sigma^{\prime}}\right)
$$

in the case of $S L_{2}$ (see formula (8.24)). 
The existence of the functions $f_{\chi}$ satisfying the function theoretic analog of the fractional Hecke property (9.10) (which we had learned from the $A$-branes) provides a consistency check for our predictions.

To summarize, we have found that the geometrically "correct" objects (corresponding to irreducible perverse sheaves $\mathcal{F}_{\chi}$, the "fractional" Hecke eigensheaves) are not the ordinary Hecke eigenfunctions, but their linear combinations (obtained by a finite Fourier transform) corresponding to a collection of Galois representations $\left\{\sigma_{\gamma}\right\}$ labeled by $\gamma \in \Gamma$. These are constructed as simple twists of $\sigma$. We note that the Fourier transform in formula (9.15) is somewhat reminiscent of the Fourier transform observed by Lusztig in the theory of character sheaves [55].

9.4.2. Non-abelian case Let us consider now the case when $\Gamma$ is nonabelian. Over $\mathbb{C}$ this means, assuming Conjecture 9.1, that the corresponding point of the Hitchin moduli space $\mathcal{M}_{\mathrm{H}}\left({ }^{\mathrm{L}} G\right)$ is not generically regular semi-simple. In this case, we expect that some of the components of the dual Hitchin fiber $\boldsymbol{F}_{b}$ have multiplicities greater than 1, which should be equal to the dimensions of the corresponding irreducible representations $R$ of $\Gamma$.

According to the conjectures of Section 9.3, transported to the realm of curves over finite fields, we have irreducible perverse sheaves $\mathcal{F}_{R}, R \in$ Irrep $(\Gamma)$, on $\operatorname{Bun}_{G}$ satisfying the fractional Hecke property (9.9). Let $f_{R}, R \in$ $\operatorname{Irrep}(\Gamma)$, be the corresponding automorphic functions. We then have an analog of formula (9.10),

$$
T_{V, x} \cdot f_{R}=\sum_{R^{\prime} \in \operatorname{Irrep}(\Gamma)} a_{V, R^{\prime}, x} f_{R \otimes R^{\prime}}, \quad R \in \operatorname{Irrep}(\Gamma)
$$

where we use the decomposition (9.2), and set

$$
a_{V, R^{\prime}, x}=\operatorname{Tr}\left(\sigma\left(\operatorname{Fr}_{x}\right), V\left(R^{\prime}\right)\right)
$$

As before,

$$
R \otimes R^{\prime}=\bigoplus_{R^{\prime \prime} \in \operatorname{Irrep}(\Gamma)}\left(R^{\prime \prime}\right)^{\oplus m_{R^{\prime \prime}}^{R, R^{\prime}}}
$$

and, by definition,

$$
f_{R \otimes R^{\prime}}=\sum_{R^{\prime \prime} \in \operatorname{Irrep}(\Gamma)} m_{R^{\prime \prime}}^{R, R^{\prime}} f_{R^{\prime \prime}}
$$


Let us find the eigenfunctions of $T_{V, x}$. They are labeled by the conjugacy classes $[\gamma]$ in $\Gamma$ and are given by the formula

$$
\widehat{f}_{[\gamma]}=\sum_{R \in \operatorname{Irrep}(\Gamma)} \operatorname{Tr}([\gamma], R) f_{R}, \quad[\gamma] \in \Gamma .
$$

The corresponding eigenvalue is

$$
A_{x,[\gamma]}=\sum_{R^{\prime} \in \operatorname{Irrep}(\Gamma)} \operatorname{Tr}\left([\gamma], R^{\prime}\right) a_{V, R^{\prime}, x}
$$

In particular,

$$
A_{x,[1]}=\sum_{R^{\prime} \in \operatorname{Irrep}(\Gamma)} \operatorname{dim}\left(R^{\prime}\right) a_{V, R^{\prime}, x}=\operatorname{Tr}\left(\sigma\left(\operatorname{Fr}_{x}\right), V\right),
$$

and so

$$
\widehat{f}_{[1]}=\sum_{R \in \operatorname{Irrep}(\Gamma)} \operatorname{dim}(R) f_{R}
$$

is a Hecke eigenfunction corresponding to $\sigma$, as expected.

The other functions $\widehat{f}_{[\gamma]}$ with $[\gamma] \neq[1]$ are also Hecke eigenfunctions, but corresponding to other homomorphisms

$$
\sigma_{[\gamma]}: W_{F} \rightarrow{ }^{\mathrm{L}} G
$$

defined by the formula

$$
\sigma_{[\gamma]}(g)=\sigma(g) \alpha_{\gamma}(g)
$$

where

$$
\alpha_{\gamma}: W_{F} \rightarrow \Gamma
$$

is the homomorphism given by the composition of res (see formula (8.26)) and the homomorphism $\mathbb{Z} \rightarrow \Gamma$ sending $1 \mapsto \gamma$, and $\gamma$ is an arbitrary element of $[\gamma]$. Clearly, the equivalence class of $\sigma_{[\gamma]}$ depends only on $[\gamma]$ and not on the choice of $\gamma$.

The corresponding Hecke eigenvalue (9.19) is

$$
A_{x,[\gamma]}=\operatorname{Tr}\left(\sigma_{\gamma}\left(\mathrm{Fr}_{x}\right), V\right)
$$

and so the function $\widehat{f}_{\gamma}$ is in fact a Hecke eigenfunction corresponding to $\sigma_{\gamma}: W_{F} \rightarrow{ }^{\mathrm{L}} G$, as desired. 
Now we can express the functions $f_{R}$ corresponding to the sheaves $\mathcal{F}_{R}$ in terms of the ordinary Hecke eigenfunctions:

$$
f_{\chi}=\frac{1}{|\Gamma|} \sum_{[\gamma] \in \Gamma} \operatorname{Tr}([\gamma], R) \widehat{f}_{[\gamma]}
$$

as in the abelian case. Thus, functions satisfying the fractional Hecke property (9.16) do exist in the non-abelian case as well. This provides a consistency check for our conjectures from Section 9.3.

\section{Gerbes}

The goal of this section is to elucidate a tricky point that arose in Section 2.2. If $r$ is a point in $\mathcal{M}_{\mathrm{H}}\left(\mathrm{SO}_{3}\right)$ corresponding to an $\mathrm{SO}_{3}$ local system with automorphism group $\Gamma=\mathbb{Z}_{2}$, then there are two branes supported at $r$, namely $\mathcal{B}_{+}$and $\mathcal{B}_{-}$. The corresponding fiber of the Hitchin fibration for $S L_{2}$ is the union of two components $\boldsymbol{F}_{1}$ and $\boldsymbol{F}_{2}$, and accordingly in the dual $A$-model, there are two $A$-branes. The central claim of this paper is that the $A$-branes $\boldsymbol{F}_{1}$ and $\boldsymbol{F}_{2}$ are dual to the $B$-branes $\mathcal{B}_{+}$and $\mathcal{B}_{-}$. But which of $\boldsymbol{F}_{1}$ and $\boldsymbol{F}_{2}$ is dual to $\mathcal{B}_{+}$and which is dual to $\mathcal{B}_{-}$? There is no natural way to decide, and indeed, $\boldsymbol{F}_{1}$ and $\boldsymbol{F}_{2}$ are exchanged by the symmetry group $Q=\mathbb{Z}_{2} \times \mathbb{Z}_{2}$. In contrast, the branes $\mathcal{B}_{+}$and $\mathcal{B}_{-}$are not equivalent; $\mathcal{B}_{+}$corresponds to the trivial representation of $\mathbb{Z}_{2}$ and $\mathcal{B}_{-}$to a nontrivial representation.

\subsection{A subtlety}

We claim that this question reflects a subtlety in the mirror symmetry of $\mathcal{M}_{\mathrm{H}}(G)$ and $\mathcal{M}_{\mathrm{H}}\left({ }^{\mathrm{L}} G\right)$ that has nothing to do with endoscopy. Let us start by asking whether the Hitchin fibration has a section. ${ }^{48}$ As explained in [33,36], a section can always be constructed if one picks a spin structure on the Riemann surface $C$, that is, a square root $K^{1 / 2}$ of the canonical bundle of $C$. Let $E=K^{-1 / 2} \oplus K^{1 / 2}$. Although $E$ is unstable, it is possible for a Higgs bundle $(E, \varphi)$ to be stable. This is so precisely if, modulo a (unique)

\footnotetext{
${ }^{48}$ For a general study of this type of question in a much more general context, see [18].
} 
automorphism of $E$,

$$
\varphi=\left(\begin{array}{ll}
0 & 1 \\
w & 0
\end{array}\right)
$$

where $w$ is a quadratic differential. Then $\operatorname{det} \varphi=-w$, so the pair $(E, \varphi)$ maps, under the Hitchin fibration, to the point in the base $\boldsymbol{B}$ determined by the quadratic differential $-w$. Since every point in $\boldsymbol{B}$ arises for a unique $(E, \varphi)$ of this form, this gives a section of the Hitchin fibration. (Higgs bundles of this form are sometimes called classical opers, reflecting their analogy to the opers of [8].)

The section obtained this way is not completely canonical, since it depends on the choice of $K^{1 / 2}$. However, the same construction (replacing $E$ by $H=\operatorname{ad}(E))$ makes sense for $S O_{3}$, and here the choice of $K^{1 / 2}$ does not matter. So for $\mathrm{SO}_{3}$, the Hitchin fibration has a natural section, but for $S L_{2}$, a choice of section depends on a choice of $K^{1 / 2}$.

This distinction is actually visible in the formulas of Section 2. The $\mathrm{SO}_{3}$ moduli space is described in Equation (3.39); the Hitchin fibration has a natural section given by $z=t=\infty$. The $S L_{2}$ moduli space is described in (3.15), and there is no natural section of the Hitchin fibration.

For any $G$, one repeats this construction, starting with a $G$-bundle that is associated to $E$ via the choice of a principal $\mathfrak{s l}_{2}$ subalgebra of $\mathfrak{g}$. Higgs bundles $(E, \varphi)$ with such an $E$ always give a section of the Hitchin fibration. This section is independent of the choice of $K^{1 / 2}$ if and only if the subgroup of $G$ that corresponds to the principal $\mathfrak{s l}_{2}$ subalgebra is $\mathrm{SO}_{3}$ rather than $S L_{2}$. (For example, if $G$ is of adjoint type, the Hitchin fibration has a natural section, and similarly if $G$ is $S L_{2 n+1}$.)

\subsection{A conundrum for mirror symmetry}

These facts lead to a puzzle for the proper statement of the mirror symmetry between $\mathcal{M}_{\mathrm{H}}\left(S O_{3}\right)$ and $\mathcal{M}_{\mathrm{H}}\left(S L_{2}\right)$.

Let ${ }^{\mathrm{L}} \boldsymbol{F}$ and $\boldsymbol{F}$ be corresponding fibers of the Hitchin fibrations of $\mathrm{SO}_{3}$ and $S L_{2}$. Naively speaking, they are dual tori, meaning that ${ }^{\mathrm{L}} \boldsymbol{F}$ parametrizes flat unitary line bundles over $\boldsymbol{F}$, and vice versa. However, there is an immediate problem: the space of flat unitary line bundles over a complex torus always has a distinguished point, corresponding to the trivial line bundle. So if ${ }^{\mathrm{L}} \boldsymbol{F}$ and $\boldsymbol{F}$ are dual tori in this sense, then each must have a distinguished point, associated with the trivial line bundle on the other. 
This contradicts the fact that, although ${ }^{\mathrm{L}} \boldsymbol{F}$ does have a distinguished point (its intersection with the section of the Hitchin fibration described above), $\boldsymbol{F}$ does not.

In fact, what is proved in [32] is not that ${ }^{\mathrm{L}} \boldsymbol{F}$ and $\boldsymbol{F}$ are dual in this naive sense, but that the abelian variety ${ }^{\mathrm{L}} \boldsymbol{F}$ is dual to the abelian variety for which $\boldsymbol{F}$ is a torsor.

For mirror symmetry between $S O_{3}$ and $S L_{2}$ theories, what this means is that the $A$-model of $\mathcal{M}_{\mathrm{H}}\left(S L_{2}\right)$ is dual, not quite to the $B$-model of $\mathcal{M}_{\mathrm{H}}\left(S_{3}\right)$, but to a slightly twisted version of this $B$-model.

A $B$-brane on a complex manifold $X$ is a coherent sheaf on $X$ (or an object of the corresponding derived category, that is, a complex of such sheaves, modulo a certain equivalence). Now let $\mathcal{G}$ be a $\mathbb{C}^{\times}$gerbe on $X$. For every $\mathcal{G}$, there is a $\mathcal{G}$-twisted version of the category of $B$-branes; for the notion of a $\mathcal{G}$-twisted sheaf, see, for example, [15, Section 2.3] or [54, Definition 2.1.2.2]. A $\mathcal{G}$-twisted coherent sheaf of rank 1 is a trivialization of $\mathcal{G}$. A direct sum of $n$ trivializations is an example of a $\mathcal{G}$-twisted sheaf of rank $n$.

For our present problem, we need a $\mathbb{C}^{\times}$gerbe over $\mathcal{M}_{\mathrm{H}}\left(S O_{3}\right)$ that is trivial but not canonically trivial. In general, let $X$ be any space and $\mathcal{L} \rightarrow X$ a complex line bundle. Then there is a canonically defined gerbe $\mathcal{G}$ whose (local) trivializations are square roots of $\mathcal{L}$. More precisely, the objects of the category associated to an open subset $U \subset X$ are pairs $(\mathcal{M}, \alpha)$, where $\mathcal{M}$ is a line bundle on $U$ and $\alpha$ is an isomorphism between $\mathcal{M}^{2}$ and $\mathcal{L}$. This gerbe $\mathcal{G}$ is trivial globally if and only if $\mathcal{L}$ has a global square root; and it can be trivialized in a unique way (up to sign) if and only if $\mathcal{L}$ has a unique global square root. $\mathcal{G}$ is a $\mathbb{C}^{\times}$gerbe, but actually it is associated with a $\mathbb{Z}_{2}$ gerbe via the embedding $\{ \pm 1\} \subset \mathbb{C}^{\times}$, so it has a natural flat gerbe connection (in physical language, it is associated with a flat $B$-field over $X$ ).

We apply this construction to the case that $X$ is $\mathcal{M}_{\mathrm{H}}(G)$ for some reductive Lie group $G$ and $\mathcal{L}$ is $K_{X}$, the canonical bundle of $X$. Considering the square roots of $K_{X}$ gives a flat $\mathbb{C}^{\times}$gerbe $\mathcal{G}$ over $X$. This gerbe is actually trivial, because $K_{X}$ does have global square roots. This point is explained in great detail in [8, Section 4], where it enters for reasons somewhat analog to our present considerations. The construction is as follows. Given any spin structure $\mathcal{S}$ on $C$ (that is, a square root of the canonical bundle of $C$ ) and a $G$-bundle $E \rightarrow C$, one considers the Pfaffian line $\mathcal{L}_{\mathcal{S}, E}$ of the Dirac operator for spin structure $\mathcal{S}$ twisted by $\operatorname{ad}(E)$. As $E$ varies, $\mathcal{L}_{\mathcal{S}, E}$ varies as the fiber of a line bundle $\mathcal{L}_{\mathcal{S}} \rightarrow \mathcal{M}_{\mathrm{H}}(G)$ that is a square root of the canonical bundle of $\mathcal{M}_{\mathrm{H}}(G)$. So the gerbe $\mathcal{G}$ has a natural trivialization for each choice of spin structure $\mathcal{S} \rightarrow C$. 
For $G$ simply connected, $\mathcal{M}_{\mathrm{H}}(G)$ is also simply-connected, so the square root of the canonical bundle obtained this way is independent of the choice of $\mathcal{S}$, up to isomorphism. This is so, for example, for $G=S L_{2}$. In this situation, $\mathcal{G}$ is canonically trivial.

In general, if $\mathcal{S}$ and $\mathcal{S}^{\prime}$ are two spin structures on $C$, the Pfaffian construction gives two square roots $\mathcal{L}_{\mathcal{S}^{\prime}}$ and $\mathcal{L}_{\mathcal{S}}$ of the canonical bundle of $\mathcal{M}_{\mathrm{H}}(G)$. They must differ by the tensor product with a line bundle $\mathcal{U}\left(\mathcal{S}^{\prime}, \mathcal{S}\right)$ of order 2 :

$$
\mathcal{L}_{\mathcal{S}^{\prime}}=\mathcal{L}_{\mathcal{S}} \otimes \mathcal{U}\left(\mathcal{S}^{\prime}, \mathcal{S}\right)
$$

Obviously, for three spin structures $\mathcal{S}, \mathcal{S}^{\prime}, \mathcal{S}^{\prime \prime} \rightarrow C$, we have

$$
\mathcal{U}\left(\mathcal{S}^{\prime \prime}, \mathcal{S}\right)=\mathcal{U}\left(\mathcal{S}^{\prime \prime}, \mathcal{S}^{\prime}\right) \otimes \mathcal{U}\left(\mathcal{S}^{\prime}, \mathcal{S}\right)
$$

A particularly simple example of this is for $G=S_{3}$. There is a natural isomorphism between $H^{1}\left(C, \mathbb{Z}_{2}\right)$ and the orbifold fundamental group $\pi_{1}\left(\mathcal{M}_{\mathrm{H}}\left(S O_{3}\right)\right)$. So there is a natural map from a line bundle $\mathcal{V} \rightarrow C$ of order 2 to an orbifold line bundle $\mathcal{T}(\mathcal{V}) \rightarrow \mathcal{M}_{\mathrm{H}}\left(S O_{3}\right)$ of order 2 . If $\mathcal{S}^{\prime}, \mathcal{S} \rightarrow C$ are two spin structures, then $\mathcal{S}^{\prime}=\mathcal{S} \otimes \mathcal{V}$ for some line bundle $\mathcal{V}$ of order 2 , and

$$
\mathcal{U}\left(\mathcal{S}^{\prime}, \mathcal{S}\right)=\mathcal{T}(\mathcal{V})
$$

a statement that is clearly compatible with Equation (10.3).

The precise statement of mirror symmetry between $\mathcal{M}_{\mathrm{H}}\left(\mathrm{SO}_{3}\right)$ and $\mathcal{M}_{\mathrm{H}}\left(S L_{2}\right)$ is as follows. The $\mathcal{G}$-twisted $\mathrm{B}$-model of $\mathrm{SO}_{3}$ is dual to the $A$-model of $S L_{2}$. And conversely, the $\mathcal{G}$-twisted $A$-model of $\mathcal{M}_{\mathrm{H}}\left(\mathrm{SO}_{3}\right)$ is dual to the $B$-model of $\mathcal{M}_{\mathrm{H}}\left(S L_{2}\right)$. In general, the $A$-model can be twisted by a flat complex gerbe, such as $\mathcal{G}$.

A similar twisting by a gerbe should also be implemented in the nonabelian Fourier-Mukai transform formulation of the geometric Langlands correspondence suggested by Beilinson and Drinfeld.

\subsection{Application}

Now let us reconsider the question with which we began: the duality between the $B$-branes $\mathcal{B}_{+}$and $\mathcal{B}_{-}$and the $A$-branes $\boldsymbol{F}_{1}$ and $\boldsymbol{F}_{2}$. Which $A$-brane corresponds to $\mathcal{B}_{+}$?

First of all, the problem only exists because the Hitchin fibration for $S L_{2}$ has no natural section. Given such a section $s$, we would be able to 
pick out a distinguished component $\boldsymbol{F}_{1}$ or $\boldsymbol{F}_{2}$ of the special Hitchin fiber, namely the one that intersects $s$.

The resolution of the problem is that the duality involves, not the ordinary $B$-model of $\mathcal{M}_{\mathrm{H}}\left(S_{3}\right)$, but the $\mathcal{G}$-twisted $B$-model. In the ordinary $B$-model, as between $\mathcal{B}_{+}$and $\mathcal{B}_{-}$, there is a distinguished one, namely the one on which the automorphism group $\mathbb{Z}_{2}$ of the $S_{3}$ local system acts trivially. But in the $\mathcal{G}$-twisted $B$-model, things are different. Although the two $B$-branes transform oppositely under $\Gamma=\mathbb{Z}_{2}$, to make sense of which transforms trivially and which transforms nontrivially, we would have to first trivialize $\mathcal{G}$.

$\mathcal{G}$, however, has no natural trivialization; rather, it has a family of trivializations depending on the choice of a spin structure on $C$. Different trivializations would give different interpretations of which of the two $B$ branes is invariant under $\Gamma$ and which is not.

Indeed, two of these trivializations differ by tensoring by one of the line bundles $\mathcal{T}(\mathcal{V})$, for some $\mathcal{V} \in H^{1}\left(C, \mathbb{Z}_{2}\right)$. If $r \in \mathcal{M}_{\mathrm{H}}\left(S O_{3}\right)$ is one of the orbifold singularities, with symmetry group $\Gamma=\mathbb{Z}_{2}$, then for suitable $\mathcal{V}$, the nontrivial element of $\Gamma$ acts on the fiber of $\mathcal{T}(\mathcal{V})$ as multiplication by -1 . When this is the case, the choice of which $B$-brane is $\Gamma$-invariant and which is not is reversed by tensoring by $\mathcal{T}(\mathcal{V})$.

\subsection{Dual symmetry groups}

For more understanding, we should describe another interpretation of some of the facts that we have exploited.

The group $Q=H^{1}\left(C, \mathbb{Z}_{2}\right)$ acts on $\mathcal{M}_{\mathrm{H}}\left(S L_{2}\right)$ in a manner familiar from Section 3.5: an element of $Q$ corresponds to a line bundle $\mathcal{V} \rightarrow C$ of order 2, which acts on a Higgs bundle $(E, \varphi)$ by $E \rightarrow E \otimes \mathcal{V}$. This gives a geometrical action of $Q$ on $\mathcal{M}_{\mathrm{H}}\left(S L_{2}\right)$, preserving its hyper-Kahler structure, so it gives an action of $Q$ on the $A$-model and the $B$-model of $\mathcal{M}_{\mathrm{H}}\left(S L_{2}\right)$.

Dually, an isomorphic group must act ${ }^{49}$ on the $B$-model and the $A$-model of the dual moduli space $\mathcal{M}_{\mathrm{H}}\left(S_{3}\right)$. The key to this is something we already exploited above: the natural correspondence $\mathcal{V} \rightarrow \mathcal{T}(\mathcal{V})$ from a line bundle $\mathcal{V} \rightarrow C$ of order 2 to a line bundle $\mathcal{T}(\mathcal{V}) \rightarrow \mathcal{M}_{\mathrm{H}}\left(S_{3}\right)$ of order 2. Since $\mathcal{T}(\mathcal{V})$ is a coherent sheaf, the tensor product with $\mathcal{T}(\mathcal{V})$ makes sense as a symmetry of the $B$-model; since it is a flat line bundle, it makes sense as a symmetry of the $A$-model.

${ }^{49}$ See [39, Section 7.2] for another explanation. 
The duality between $\mathcal{M}_{\mathrm{H}}\left(S L_{2}\right)$ and $\mathcal{M}_{\mathrm{H}}\left(S O_{3}\right)$ exchanges the action of $Q$ on the $A$-and $B$-models of $\mathcal{M}_{\mathrm{H}}\left(S L_{2}\right)$ coming from its geometrical action on this space with the action of $Q$ on the $\mathcal{G}$-twisted $B$-and $A$-models of $\mathcal{M}_{\mathrm{H}}\left(S_{3}\right)$ by tensor product.

$Q$ exchanges the two $A$-branes supported on the special Hitchin fiber of $\mathcal{M}_{\mathrm{H}}\left(S L_{2}\right)$ for geometrical reasons. It exchanges the two $B$-branes supported at the orbifold singularity because the nontrivial element of $\Gamma=\mathbb{Z}_{2}$ acts as -1 on the relevant fiber of some of the line bundles $\mathcal{T}(\mathcal{V})$.

\subsection{Relation to the usual statement of geometric langlands}

In this paper, in order to explore endoscopy, we have primarily compared the $\mathcal{G}$-twisted $B$-model of $S_{3}$ to the $A$-model of $S L_{2}$. However, it is also of interest to compare the $B$-model of $S L_{2}$ to the $\mathcal{G}$-twisted $A$-model of $\mathrm{SO}_{3}$. What does the $\mathcal{G}$-twisting do in that context?

It is shown in [39, Section 11] that, for any $G$, an $A$-brane on $\mathcal{M}_{\mathrm{H}}(G)$ is equivalent to a twisted $\mathcal{D}$-module on $\mathcal{M}(G)$, the moduli space of $G$-bundles. Here $^{50}$ a twisted $\mathcal{D}$-module is a sheaf of modules for a sheaf of algebras that we call $\mathcal{D}^{*}$, the differential operators acting on a square root of the canonical bundle $K_{\mathcal{M}}$ of $\mathcal{M}$. The sheaf of algebras $\mathcal{D}^{*}$ does not depend on a global choice of square root of $K_{\mathcal{M}}$ (or even on the global existence of such a square root, though it does in fact exist).

There is a slight tension between this and the usual statement of geometric Langlands duality: the right-hand side of the duality is supposed to involve an ordinary $\mathcal{D}$-module, rather than a twisted $\mathcal{D}$-module.

Now if there is a canonical global square root of $K_{\mathcal{M}}$, then this distinction is inessential. Given such a line bundle, we can consider the differential operators that map $\mathcal{O}_{\mathcal{M}}$ to $K_{\mathcal{M}}^{1 / 2}$ and the sheaf of such operators is a "bimodule" for the pair of (sheaves of) algebras $\mathcal{D}$ and $\mathcal{D}^{*}$, i.e., the ordinary and twisted differential operators. This bi-module is a "Morita equivalence bimodule" that establishes an equivalence between the categories of ordinary and twisted $\mathcal{D}$-modules.

For $S L_{2}$, there is such a canonical choice of $K_{\mathcal{M}}^{1 / 2}$, but for $S O_{3}$ there is not. So a $\mathcal{D}^{*}$-module on $\mathcal{M}\left(\mathrm{SO}_{3}\right)$, such as we would get from an $A$-brane of $\mathcal{M}_{\mathrm{H}}\left(\mathrm{SO}_{3}\right)$, is not canonically the same thing as an ordinary $\mathcal{D}$-module on $\mathcal{M}\left(\mathrm{SO}_{3}\right)$, such as we expect in the geometric Langlands program.

\footnotetext{
${ }^{50}$ For simplicity, we consider only the unramified case. Ramification leads to a further twisting that does not affect our main claim.
} 
What reconciles the two viewpoints is that the $A$-model on $\mathcal{M}_{\mathrm{H}}\left(\mathrm{SO}_{3}\right)$ that arises in $S$-duality is $\mathcal{G}$-twisted. While an ordinary $A$-brane maps to a $\mathcal{D}^{*}$-module on $\mathcal{M}\left(S_{3}\right)$, a $\mathcal{G}$-twisted $A$-brane maps to a $\mathcal{G}$-twisted $\mathcal{D}^{*}$ module on the same space. But a $\mathcal{G}$-twisted $\mathcal{D}^{*}$-module on $\mathcal{M}\left(\mathrm{SO}_{3}\right)$ maps canonically to an ordinary $\mathcal{D}$-module on the same space. The reason for this is that although a square root $K_{\mathcal{M}}^{1 / 2}$ does not exist canonically as a line bundle, it does exist canonically — and tautologically — as a trivialization of the gerbe $\mathcal{G}$.

We have described this for the dual pair of groups $\mathrm{SO}_{3}$ and $\mathrm{SL}_{2}$ that has been our main example. More generally, for any reductive group $G$, one defines the gerbe $\mathcal{G}$ of square roots of the canonical bundle. Given any dual pair ${ }^{\mathrm{L}} G$ and $G$, the underlying gauge theory duality is an isomorphism between the $\mathcal{G}$-twisted $B$-model of ${ }^{\mathrm{L}} G$ and the $\mathcal{G}$-twisted $A$-model of $G$ (and vice versa). In our example, we have seen the twisting on only one side, simply because the gerbe $\mathcal{G}$ is canonically trivial for $S L_{2}$.

\section{Appendix: $L$-packets for $S L_{2}$.}

In this Appendix, we sketch a proof of Theorem 7.1 using the Whittaker functions. The construction of automorphic functions for $G L_{2}$ via a Fourier transform of Whittaker functions was introduced by Jacquet and Langlands [37] using a result of Weil [77]. In what follows, we use the presentation and notation of [23].

We will fix a nonzero rational differential $\omega$ on $C$ and denote by $\delta=$ $\sum_{x} \delta_{x}[x]$ its divisor of zeros and poles. Let $\psi: \mathbb{F}_{q} \rightarrow \overline{\mathbb{Q}}_{\ell}^{\times}$be a nontrivial additive character. It gives rise to a character $\Psi: \mathbb{A}_{F} \rightarrow \overline{\mathbb{Q}}_{\ell}^{\times}$defined by formula

$$
\Psi\left(\left(f_{x}\right)\right)=\prod_{x \in C} \psi\left(\operatorname{Tr}_{k_{x} / \mathbb{F}_{q}}\left(\operatorname{Res}_{x}\left(f_{x} \omega\right)\right)\right)
$$

By residue formula, its value on $F \subset \mathbb{A}_{F}$ is equal to 1 . Hence $\Psi$ gives rise to a character of $\mathbb{A}_{F} / F$.

Let $B \subset G L_{2}$ be the Borel subgroup of upper triangular matrices. Denote by $V$ the space of locally constant functions $f: G L_{2}\left(\mathbb{A}_{F}\right) \rightarrow \overline{\mathbb{Q}}_{\ell}$ such that

(1) $f(\alpha x)=f(x)$ for all $x \in G L_{2}\left(\mathbb{A}_{F}\right), \alpha \in B(F)$;

(2) $\int_{\mathbb{A}_{F} / F} f\left(\left(\begin{array}{ll}1 & z \\ 0 & 1\end{array}\right) x\right) d z=0$ for all $x \in G L_{2}\left(\mathbb{A}_{F}\right)$;

(3) $f(x u)=f(x)$ for all $x \in G L_{2}\left(\mathbb{A}_{F}\right), u \in G L_{2}\left(\mathcal{O}_{F}\right)$. 
Denote by $W$ the space of locally constant functions $\phi: G L_{2}\left(\mathbb{A}_{F}\right) \rightarrow \overline{\mathbb{Q}}_{\ell}$ such that

(1') $\phi\left(\left(\begin{array}{ll}a & z \\ 0 & a\end{array}\right) x\right)=\Psi(z) \phi(x)$ for all $x \in G L_{2}\left(\mathbb{A}_{F}\right), z \in \mathbb{A}_{F}, a \in F^{\times}$;

$\left(2^{\prime}\right) f(x u)=f(x)$ for all $x \in G L_{2}\left(\mathbb{A}_{F}\right), u \in G L_{2}\left(\mathcal{O}_{F}\right)$.

Define maps between these two spaces by the formulas

$$
\begin{aligned}
& f \in V \mapsto \phi \in W ; \quad \phi(x)=\int_{\mathbb{A}_{F} / F} f\left(\left(\begin{array}{ll}
1 & z \\
0 & 1
\end{array}\right) x\right) \Psi(-z) d z, \\
& \phi \in W \mapsto f \in V ; \quad f(x)=\sum_{a \in F^{\times}} \phi\left(\left(\begin{array}{ll}
a & 0 \\
0 & 1
\end{array}\right) x\right) .
\end{aligned}
$$

According to [37], these maps are mutually inverse isomorphisms. In addition, they intertwine the (right) action of the spherical Hecke algebra of $G L_{2}$ on both spaces. Therefore, the spaces of Hecke eigenfunctions in the two spaces are isomorphic. The corresponding eigenvalues are determined by a collection of $G L_{2}$ conjugacy classes $\gamma_{x}, x \in C$.

It is known that, for any such collection $\left(\gamma_{x}\right)_{x \in C}$, the space of Hecke eigenfunctions in $W$ is spanned by the so-called Whittaker function. To write down an explicit formula for this function [77], let us denote by $V_{m, k}, m \geq k$, the irreducible representation of $G L_{2}$ with the highest weight $(m, k)$, that is $\operatorname{Sym}^{m-k} V \otimes(\operatorname{det} V)^{\otimes k}$, where $V$ is the defining two-dimensional representation. Note that using the above conditions (1') and (2'), a function $\phi \in W$ is uniquely determined by its values on elements of the form

$$
\left(\left(\begin{array}{cc}
t_{x}^{m_{x}} & 0 \\
0 & t_{x}^{k_{x}}
\end{array}\right)\right)_{x \in C} \in G L_{2}\left(\mathbb{A}_{F}\right)
$$

(here, as before, $t_{x}$ denotes a uniformizer, that is, a formal coordinate, at $x$ ). Given a collection $\left(\gamma_{x}\right)_{x \in X}$ of conjugacy classes, the corresponding Hecke eigenfunction in $W$ is then determined (up to some inessential nonzero factors) by the formula

$$
\phi\left(\left(\begin{array}{cc}
t_{x}^{m_{x}} & 0 \\
0 & t_{x}^{k_{x}}
\end{array}\right)\right)=\prod_{x \in C} \operatorname{Tr}\left(\gamma_{x}, V_{m_{x}+\delta_{x}, k_{x}}\right)
$$

(if $m_{x}+\delta_{x}<k_{x}$ for some $x \in C$, then the right-hand side is equal to 0 , by definition). 
A cuspidal automorphic Hecke eigenfunction for $G L_{2}$ with the eigenvalues corresponding to a collection $\left(\gamma_{x}\right)_{x \in X}$ as above is, by definition, a Hecke eigenfunction that satisfies the above conditions (2) and (3), and a stronger condition than (1); namely, that $f(\alpha x)=f(x)$ for all $x \in G L_{2}\left(\mathbb{A}_{F}\right)$, $\alpha \in G L_{2}(F)$. In other words, it is a function on the double quotient

$$
\operatorname{Bun}_{G L_{2}}\left(\mathbb{F}_{q}\right)=G L_{2}(F) \backslash G L_{2}\left(\mathbb{A}_{F}\right) / G L_{2}\left(\mathcal{O}_{F}\right) .
$$

The above results that imply that, if it exists, this function must be obtained by applying the transform (A.2) to the Whittaker function with the eigenvalues $\left(\gamma_{x}\right)_{x \in C}$. In particular, if exists, it is unique up to a scalar multiple.

According to the results of Drinfeld [22, 23], if $\widetilde{\sigma}: W_{F} \rightarrow G L_{2}$ is irreducible and unramified, then the vector space of Hecke eigenfunction on (A.5) with respect to $\widetilde{\sigma}$ is one-dimensional and consists of cuspidal functions. Therefore a generator of this space is given by the operator (A.2) applied to the Whittaker function with the eigenvalues $\left(\widetilde{\sigma}\left(\operatorname{Fr}_{x}\right)\right)$. Let us denote this automorphic function by $f_{\widetilde{\sigma}}$ and the corresponding Whittaker function by $W_{\widetilde{\sigma}}$.

Now we switch from $G L_{2}$ to $S L_{2}$. Recall that we would like to find the dimension of the space of Hecke eigenfunctions on $\operatorname{Bun}_{S L_{2}}^{\mathcal{O}(D)}\left(\mathbb{F}_{q}\right)$, which is the double quotient (7.12) (here, as before, $D$ is a finite subset of $C$, which we view as an effective divisor). We embed this component into the double quotient (A.5) by sending $\left(g_{x}\right) \mapsto\left(\bar{g}_{x}\right)$, where

$$
\bar{g}_{x}=g_{x}\left(\begin{array}{cc}
t_{x} & 0 \\
0 & 1
\end{array}\right)
$$

if $x \in D$ and $\bar{g}_{x}=g_{x}$, otherwise. We will assume that $\sigma: W_{F} \rightarrow O_{2} \subset P G L_{2}$ may be lifted to a homomorphism $\widetilde{\sigma}: W_{F} \rightarrow G L_{2}$. It is easy to see that the space of Hecke eigenfunctions on $\operatorname{Bun}_{S L_{2}}^{\mathcal{O}(D)}\left(\mathbb{F}_{q}\right)$ with respect to $\sigma$ is equal to the restriction of the space of Hecke eigenfunctions on (A.5) to the image of this embedding with respect to $\widetilde{\sigma}$.

Observe that for generic $\sigma$ of the above form the representation $\widetilde{\sigma}$ will be irreducible. Thus, to determine whether the space of Hecke eigenfunctions on $\operatorname{Bun}_{S L_{2}}^{\mathcal{O}(D)}\left(\mathbb{F}_{q}\right)$ with respect to $\sigma$ is zero- or one-dimensional, we need to determine whether the restriction of the function $f_{\widetilde{\sigma}}$ constructed above to the image of $\operatorname{Bun}_{S L_{2}}^{\mathcal{O}(D)}\left(\mathbb{F}_{q}\right)$ is zero or not. This, in turn, is determined by whether the Whittaker function $W_{\widetilde{\sigma}}$ is equal to zero on all elements of the 
form (A.3), where the divisor

$$
\sum_{x \in C}\left(m_{x}+k_{x}\right)[x]
$$

is linearly equivalent to $D$.

Let $\alpha: W_{F} \rightarrow \mathbb{Z}_{2}$ be the quadratic character obtained as the composition of $\sigma$ and the homomorphism $O_{2} \rightarrow \mathbb{Z}_{2}$. It corresponds to a quadratic extension $E / F$, which we have called in Section 8.8 affiliated with $\sigma$. We have

$$
\alpha\left(\mathrm{Fr}_{x}\right)=\left\{\begin{aligned}
1, & x \text { is split in } E \\
-1, & x \text { is nonsplit in } E
\end{aligned}\right.
$$

For any divisor $M=\sum_{x \in C} M_{x}[x]$ on $C$, let us set

$$
\langle M\rangle=\sum_{x \text { nonsplit in } E} M_{x}
$$

Recall that we have the divisor $\delta$ of the differential $\omega$ used in the definition of the character $\Psi$, and we have the divisor $D=\sum_{x} D_{x}[x]$, with $D_{x}=0$ or 1 .

We claim that the values of the Whittaker function $W_{\widetilde{\sigma}}$ are zero on all elements of the form (A.3), with

$$
\sum_{x \in C}\left(m_{x}+k_{x}\right)[x]
$$

linearly equivalent to $D$, if and only if $\langle\delta+D\rangle$ is odd. Indeed, this set contains the element $\left(g_{x}\right)$, where

$$
g_{x}=\left(\begin{array}{cc}
t_{x} & 0 \\
0 & 1
\end{array}\right), x \in D
$$

and $g_{x}=1$, otherwise. According to formula (A.4), the value of $W_{\widetilde{\sigma}}$ on this element is equal to

$$
\prod_{x \in C} \operatorname{Tr}\left(\widetilde{\sigma}\left(\mathrm{Fr}_{x}\right), V_{\delta_{x}+D_{x}, 0}\right)
$$


If $\langle\delta+D\rangle$ is odd, then there is at least one nonsplit point $y$ such that $\delta_{y}+D_{y}$ is odd. But since $\widetilde{\sigma}\left(\mathrm{Fr}_{y}\right)$ is conjugate to a scalar multiple of matrix (7.5) in this case, we find that

$$
\operatorname{Tr}\left(\widetilde{\sigma}\left(\operatorname{Fr}_{y}\right), V_{\delta_{y}+D_{y}, 0}\right)=0
$$

Therefore, $W_{\widetilde{\sigma}}$ is equal to 0 at this point. All other points that we need to check have the form (A.3), with

$$
\sum_{x \in C}\left(m_{x}+k_{x}\right)[x]=D+(F)
$$

where

$$
(F)=\sum_{x \in C} n_{x}[x]
$$

is the divisor of zeros and poles of a rational function $F$ on $C$. But it follows from the abelian class field theory [67] that for any rational function $F$ on $C$ we have

$$
\prod_{x \in C} \alpha\left(\mathrm{Fr}_{x}\right)^{n_{x}}=\prod_{x \text { nonsplit in } E}(-1)^{n_{x}}=1
$$

Therefore, for any element (A.3) satisfying the above conditions, there again exists at least one nonsplit point $z \in C$ such that $m_{z}+\delta_{z}-k_{z}$ is odd. Formula (A.4) then shows that the value of $W_{\widetilde{\sigma}}$ on all such elements is 0 . Therefore, we find that in this case the restriction of $f_{\widetilde{\sigma}}$ to the image of $\operatorname{Bun}_{S L_{2}}^{\mathcal{O}(D)}\left(\mathbb{F}_{q}\right)$ in (A.5) is equal to 0. Hence there are no nonzero Hecke eigenfunctions on $\operatorname{Bun}_{S L_{2}}^{\mathcal{O}(D)}\left(\mathbb{F}_{q}\right)$ with respect to $\sigma$, which is what we wanted to show.

On the other hand, if $\langle\delta+D\rangle$ is even, then it is easy to see that the restriction of $f_{\widetilde{\sigma}}$ to the image of $\operatorname{Bun}_{S L_{2}}^{\mathcal{O}(D)}\left(\mathbb{F}_{q}\right)$ is nonzero. Hence the corresponding space of Hecke eigenfunctions on $\operatorname{Bun}_{S L_{2}}^{\mathcal{O}(D)}\left(\mathbb{F}_{q}\right)$ is one-dimensional.

To complete the proof of Theorem 7.1, it remains to observe that

$$
\langle\delta\rangle=\sum_{x \text { nonsplit in } E} \delta_{x}
$$


is always even (see Theorem 13 in [76], Ch. XIII, Section 12). ${ }^{51}$ Hence the parity of $\langle\delta+D\rangle$ is the same as that of $\langle D\rangle$, which is the same as the number of elements in the subset $S \subset D$ consisting of all nonsplit points of $D$.

\section{Acknowledgments}

E.F. wishes to express his gratitude to R. Langlands for conveying his insights on the endoscopy. He also thanks J. Arthur, D. Kazhdan, R. Kottwitz, B.-C. Ngô, M. Olsson, T. Pantev, B. Poonen and K. Ribet for their helpful answers to various questions related to this paper, and D. Arinkin, A. Beilinson, R. Bezrukavnikov and V. Lafforgue for stimulating discussions. E.W. similarly thanks T. Hausel, D. R. Morrison, T. Pantev, I. M. Singer and C. Vafa. We are also grateful to S. Gukov for his comments on the draft. Supported in part by DARPA and AFOSR through the Grant FA9550-07-1-0543. Supported in part by NSF Grant PHY-0503584

\section{References}

[1] M. Aganagic, R. Dijkgraaf, A. Klemm, M. Mariño and C. Vafa, Topological strings and integrable hierarchies, Commun. Math. Phys. 261 (2006), 451-516, hep-th/0312085.

[2] D. Arinkin, Moduli of connections with a small parameter on a curve, Preprint, math.AG/0409373.

[3] S. Arkhipov and D. Gaitsgory, Another realization of the category of modules over the small quantum group, Adv. Math. 173 (2003), 114-143.

[4] J. Arthur, The principle of functoriality, Bull. AMS 40 (2002), 39-53.

[5] J. Arthur, An introduction to the trace formula, Clay Mathematics Proceedings, 4, AMS, Providence, 2005.

[6] P. Aspinwall, Enhanced gauge symmetry and K3 surfaces, Phys. Lett. B357 (1995), 329-334, hep-th/9507012.

[7] A. Beilinson, J. Bernstein and P. Deligne, Faisceaux pervers, Astérisque, Soc. Math. France, Paris 100 (1982).

${ }^{51}$ We thank B. Poonen for pointing out this reference. 
[8] A. Beilinson and V. Drinfeld, Quantization of Hitchin's integrable system and Hecke eigensheaves, Preprint, available at www.math. uchicago.edu/ arinkin/langlands

[9] M. Bershadsky, A. Johansen, V. Sadov and C. Vafa, Topological reduction of 4-D SYM to 2-D sigma models, Nucl. Phys. B448 (1995), 166-186.

[10] O. Biquard, Fibrs de Higgs Et Connexions Intgrables: Le Cas Logarithmique (Diviseur Lisse), Ann. Sci. Cole Norm. Sup. 30(4) (1997), 41-96.

[11] O. Biquard and P. Boalch, Wild nonabelian Hodge theory on curves, Compos. Math. 140 (2004), 179-204.

[12] A. Braverman and D. Gaitsgory, Geometric Eisenstein series, Invent. Math. 150 (2002), 287-384.

[13] R. Cluckers and F. Loeser, Ax-Kochen-Ershov theorems for p-adic integrals and motivic integration, in eds. F. Bogomolov and Yu. Tschinkel, Geometric methods in algebra and number theory Progress in Mathematics, 235, pp. 109-137, Birkhäuser, Boston, 2005.

[14] J.-F. Dat, Lemme Fondamental Et Endoscopie, Une Approche Géométrique [d'après Gérard Laumon and Ngô Bao-Châu], Séminaire Bourbaki, no. 940, November 2004.

[15] A.J. de Jong, A result of Gabber, Preprint.

[16] P. Deligne, La Conjecture De Weil. II, Publ. Math. IHES 52 (1980), 137-252.

[17] R. Dijkgraaf, L. Hollands, P. Sulkowski and C. Vafa, Supersymmetric gauge theories, intersecting branes, and free fermions, Preprint, arXiv:hep-th/0709.4446.

[18] R. Donagi and D. Gaitsgory, The gerbe of Higgs bundles, Transform. Groups 7 (2002), 109-153.

[19] R. Donagi and E. Witten, Supersymmetric Yang-Mills theory and integrable systems, Nucl. Phys. B460 (1994), 299-334, hep-th/9510101.

[20] R. Donagi and T. Pantev, Langlands duality for Hitchin systems, arXiv:math.AG/0604617.

[21] M.R. Douglas and G. Moore, D-branes, quivers, and ALE instantons, arXiv:hep-th/9603167. 
[22] V.G. Drinfeld, Langlands conjecture for GL(2) over function field, Proceedings of International Congress of Mathematics (Helsinki, 1978), 565-574; Moduli Varieties of F-sheaves, Funct. Anal. Appl. 21 (1987), 107-122; The proof of Petersson's conjecture for GL(2) over a global field of characteristic p, Funct. Anal. Appl. 22 (1988), 28-43.

[23] V.G. Drinfeld, Two-dimensional $\ell$-adic representations of the fundamental group of a curve over a finite field and automorphic forms on GL(2), Amer. J. Math. 105 (1983), 85-114.

[24] D.S. Freed and E. Witten, Anomalies in string theory with D-branes, Asian J. Math. 3 (1999), 819-851, hep-th/9907189.

[25] E. Frenkel, Lectures on the Langlands program and conformal field theory, arXiv:hep-th/0512172.

[26] E. Frenkel, Ramifications of the geometric Langlands program, arXiv: math/0611294.

[27] E. Frenkel and D. Gaitsgory, Local geometric Langlands correspondence and affine Kac-Moody algebras, arXiv:math.RT/0508382.

[28] E. Frenkel, D. Gaitsgory and K. Vilonen, On the geometric Langlands conjecture, J. AMS 15 (2001), 367-417.

[29] M. Goresky, R. Kottwitz and R. Macpherson, Homology of affine Springer fibers in the unramified case, Duke Math. J. 121 (2004), 509-561.

[30] S. Gukov and E. Witten, Gauge theory, ramification, and the geometric Langlands program, arXiv:hep-th/0612073.

[31] J. A. Harvey, G. W. Moore and A. Strominger, Reducing $S$ duality to T duality, Phys. Rev. D52 (1995), 7161-7167.

[32] T. Hausel and M. Thaddeus, Mirror symmetry, Langlands duality, and the Hitchin system, arXiv:math.AG/0205236.

[33] N. Hitchin, The self-duality equations on a Riemann surface, Proc. London Math. Soc. 55(3) (1987), 59-126.

[34] N. Hitchin, Stable bundles and integrable systems, Duke Math. J. 54 (1987), 91-114.

[35] N. Hitchin, Langlands duality and $G_{2}$ spectral curves, arXiv:math.AG/ 0611524. 
[36] N. Hitchin, Lie groups and Teichmuller space, Topology 31 (1992), 449-473.

[37] H. Jacquet and R. Langlands, Automorphic forms on GL(2), Lecture Notes in Mathematics, 114, Springer-Verlag, Berlin, 1970.

[38] A. Kapustin and D. Orlov, Remarks on A-branes, mirror symmetry, and the Fukaya category, J. Geom. Phys. 48 (2003), 84-99, arXiv:hepth/019098.

[39] A. Kapustin and E. Witten, Electric-magnetic duality and the geometric Langlands program, Comm. Number Theory Phys. 1 (2007), 1-236, arXiv:hep-th/0604151.

[40] R. Kottwitz, Stable trace formula: cuspidal tempered terms, Duke Math. J. 51 (1984), 611-650.

[41] P. Kronheimer, The construction of ALE spaces as Hyper-Kahler quotients, J. Differential Geom. 29 (1989), 665-683.

[42] J.-B. Labesse and R.P. Langlands, L-indistinguishability for $S L(2)$, Can. J. Math. 31 (1979), 726-785.

[43] L. Lafforgue, Chtoucas de drinfeld et correspondance de Langlands, Invent. Math. 147 (2002), 1-241.

[44] R.P. Langlands, Problems in the theory of automorphic forms, Lecture Notes in Mathematics, 170, Springer Verlag, Berlin, 1970, 18-61.

[45] R.P. Langlands, Les Débuts D'une Formule Des Traces Stable, Publications Mathématiques de l'Université Paris VII, 13, Université de Paris VII, U.E.R. de Mathématiques, Paris, 1983.

[46] R.P. Langlands, Representation theory: its rise and its role in number theory, Proceedings of the Gibbs Symposium (New Haven, CT, 1989), AMS, 1990, 181-210.

[47] R.P. Langlands, Reflections on receiving the Shaw prize, September 2007.

[48] G. Laumon, Transformation De Fourier, Constantes D'équations Fonctionelles Et Conjecture De Weil, Publ. IHES 65 (1987), $131-210$.

[49] G. Laumon, Correspondance De Langlands Géométrique Pour Les Corps De Fonctions, Duke Math. J. 54 (1987), 309-359. 
[50] G. Laumon, Faisceaux Automorphes Liés Aux Séries d'Eisenstein, in Automorphic forms, shimura varieties, and L-functions, Vol. I (Ann Arbor, MI, 1988), 227-281, Perspect. Math., 10, Academic Press, 1990.

[51] G. Laumon, Transformation De Fourier Généralisée, Preprint, arXiv: alg-geom/9603004.

[52] G. Laumon, Sur Le Lemme Fondamental Pour Les Groupes Unitaires, Preprint, arXiv:math/0212245.

[53] G. Laumon and B.-C. Ngô, Le Lemme Fondamental Pour Les Groupes Unitaires, Preprint, arXiv:math/0404454.

[54] M. Lieblich, Moduli of twisted sheaves, Preprint, math.AG/0411337.

[55] G. Lusztig, Character sheaves, V, Adv. Math. 61 (1986), 103-155.

[56] S. Lysenko, Geometric Waldspurger periods, Preprint, arXiv:math/ 0510110, Compos. Math. (to appear).

[57] S. Lysenko, Geometric theta-lifting for the dual pair $S O_{2 m}, S p_{2 n}$, Preprint, arXiv:math/0701170.

[58] I. Mirković and K. Vilonen, Geometric Langlands duality and representations of algebraic groups over commutative rings, math.RT/0401222.

[59] T. Mochizuki, Kobayashi-Hitchin correspondence for tame Harmonic bundles and an application, Asterisque, Soc. Math. France, Paris No. 309 (2006).

[60] D. Mumford, Prym Varieties I, in Contributions to analysis, A Collection of Papers Dedicated to Lipman Bers, Academic Press, New York, 1974, 325-350.

[61] D. Nadler, Microlocal branes are constructible sheaves, Preprint, arXiv:math/0612399.

[62] D. Nadler and E. Zaslow, Constructible sheaves and the Fukaya category, Preprint, arXiv:math/0604379.

[63] H. Nakajima, Hyper-Kahler structures on moduli spaces of parabolic higgs bundles on Riemann surfaces, in ed. M. Maruyama, Moduli of Vector Bundles Lecture Notes in Pure and Applied Math, 179, Marcel Dekker, New York, 1996.

[64] B.-C. Ngô, Fibration De Hitchin Et Endoscopie, Invent. Math. 164 (2006), 399-453. 
[65] B.-C. Ngô, Le Lemme Fondamental Pour Les Algèbres De Lie, Preprint, http://www.math.u-psud.fr/ ngo/LFLS.pdf

[66] M. Rothstein, Connections on the total Picard sheaf and the KP hierarchy, Acta Appl. Math. 42 (1996), 297-308.

[67] J.-P. Serre, Abelian groups and class fields, Springer-Verlag, Berlin, 1988.

[68] C. Simpson, Harmonic bundles on noncompact curves, J. Amer. Math. Soc. 3 (1990), 713-770.

[69] C. Simpson, Higgs bundles and local systems, Pub. Math. IHES 75 (1992), 5-95.

[70] C. Simpson, Moduli of Representations of the fundamental group of a smooth projective variety I, Pub. Math. IHES 79 (1994), 47-129.

[71] N. Seiberg and E. Witten, Monopoles, duality and chiral symmetry breaking in $\mathcal{N}=2$ supersymmetric QCD, Nucl. Phys. B431 (1994), 484-550, hep-th/9408099.

[72] N. Seiberg and E. Witten, Gauge theory and compactification to three dimensions, The mathematical beauty of physics (Saclay, 1996), 333366 , hep-th/9607163.

[73] A. Strominger, S.-T. Yau and E. Zaslow, Mirror symmetry is T-duality, Nucl. Phys. B479 (1996), 243-259.

[74] J.-L. Waldspurger, Le Lemme Fondamental Implique Le Transfert, Comp. Math. 105 (1997), 153-236.

[75] J.-L. Waldspurger, Endoscopie Et Changement De Caractéristique, Preprint, http://www.institut.math.jussieu.fr/ waldspur/endoscopietordue.pdf

[76] A. Weil, Basic number theory, Die Grundlehren der mathematischen Wissenschaften, 144, Springer-Verlag, Berlin, 1967.

[77] A. Weil, Dirichlet series and automorphic forms, Lecture Notes in Mathematics, 189, Springer, Berlin, 1971.

[78] E. Witten, Baryons and branes in anti de sitter space, JHEP 9807 (1998), 006 hep-th/9805112.

[79] E. Witten, Gauge theory and wild ramification, arXiv:0710.0631. 
[80] E. Witten, Solutions of four-dimensional field theories via $M$ theory, Nucl. Phys. B500 (1997), 3-42, hep-th/9703166.

Department of Mathematics

University of CALIFornia

BERKELEY

CA 94720

USA

E-mail address: frenkel@math.berkeley.edu

School of Natural Sciences

Institute for Advanced Study

PRINCETON

NJ 08540

USA

E-mail address: witten@ias.edu

Received December 11, 2007 